

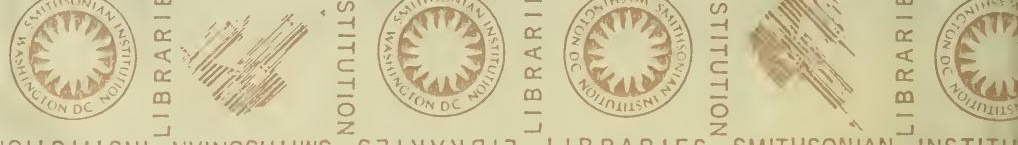
NOIINIIISNI NHINOSHLIWS ${ }^{-1} S \exists I Y \forall Y 817^{-1}$ LIBRARIES $^{2}$ SMITHSONIAN $^{-1}$ INSTITU
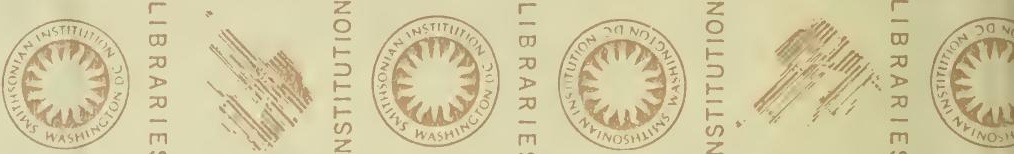

LIBRARIES SMITHSONIAN

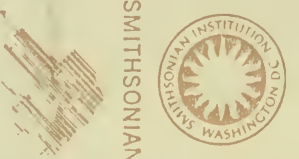

INSTITUTION NOILIIISNI

NHINOSHLIWS S $\exists I Y \forall r$

NOIIRHISNI NYINOSHIIWS
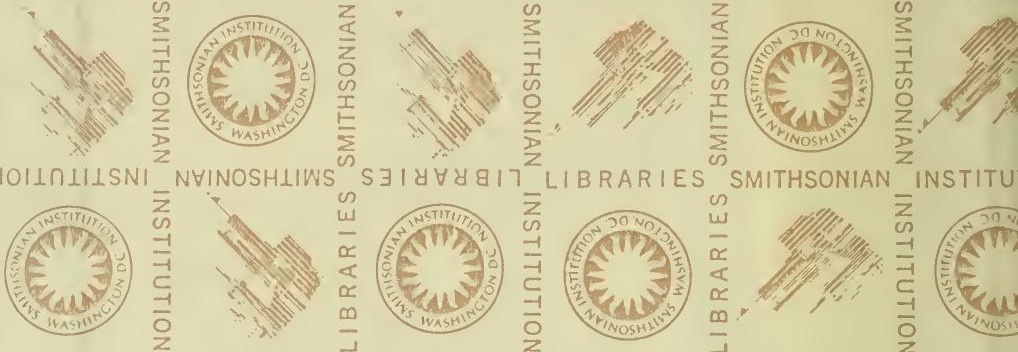

LIBRARIES SMITHSONIAN

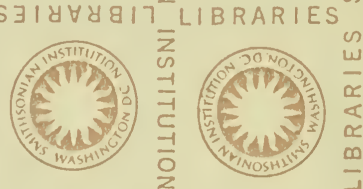

SMITHSONIAN
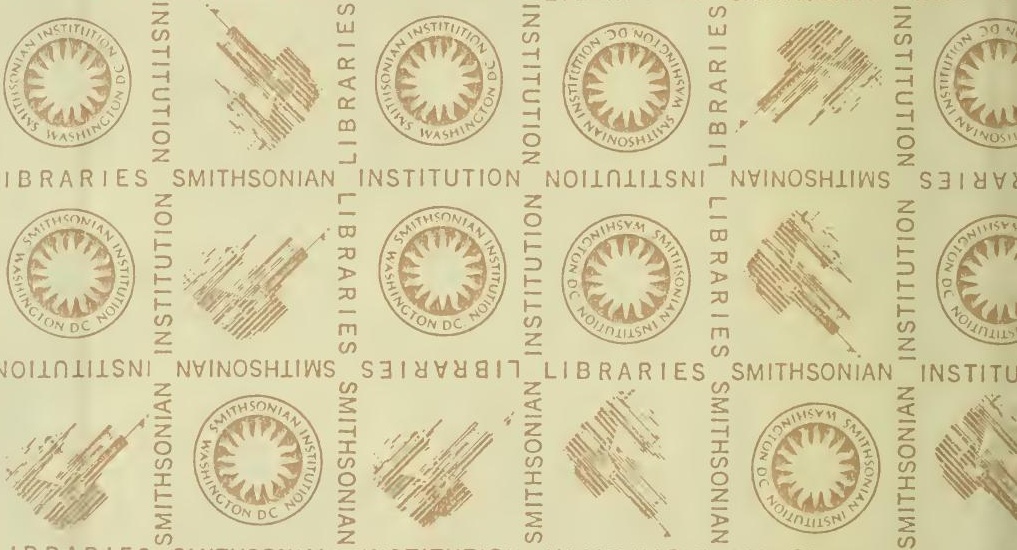

LIBRARIES SMITHSONIAN
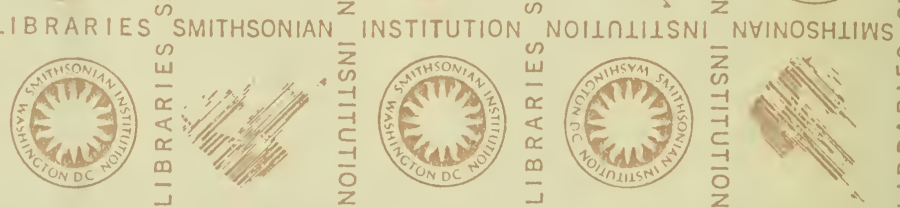

$S \exists I d \forall$

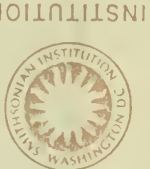
NYINOSHLIWS
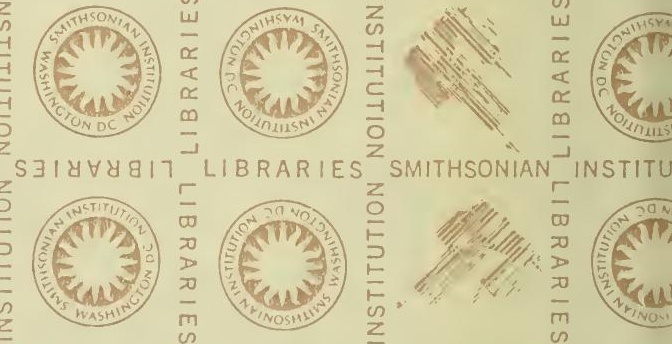

LIBRARIES SMITHSONIAN
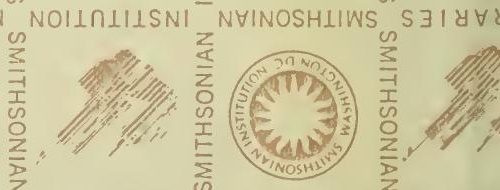
NOILOHIISNI NHINOSHIIW

SMITHSONIAN

INSTITU

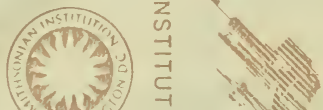

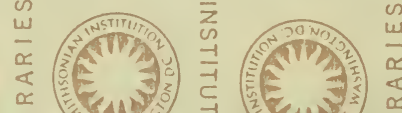







\section{THE TYPE-SPECIES OF THE NORTH AMERICAN GENERA OF DIPTERA}

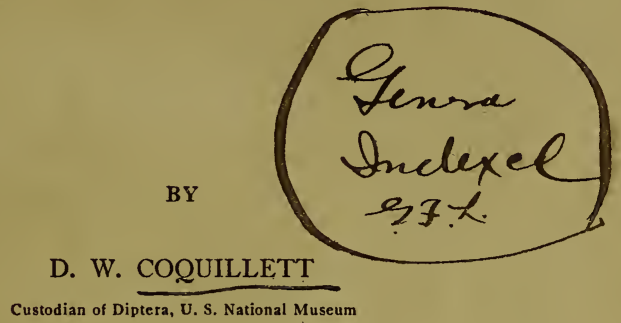

No. 1719.-From the Proceedings of the United States National Museum, Vol. 37, pages 499-647

Published August 4, 1910

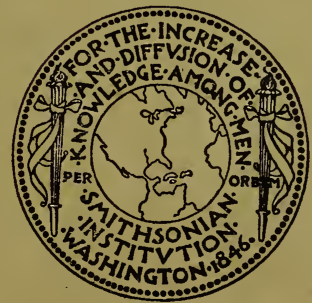

Washington

Government Printing Office 1910 Q 

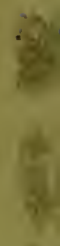

8

k
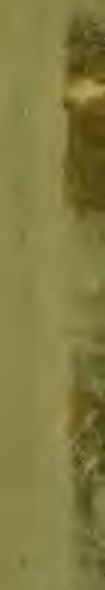


$$
\begin{aligned}
& 5: 35.1 \\
& A_{1} C 7 \\
& \text { Ent. }
\end{aligned}
$$

\title{
THE TYPE-SPECIES OF THE NORTH AMERICAN
}

\section{GENERA OF DIPTERA}

\author{
$\mathrm{BY}$ \\ D. W. COQUILLETT \\ Custodian of Diptera, U. S. National Museum
}

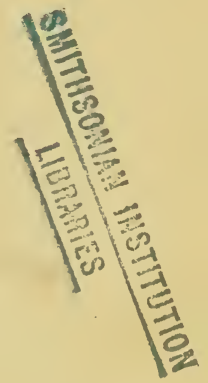

No. 1719.--From the Proceedings of the United States National Museum, Vol. 37, pages 499-647

Published August 4, 1910

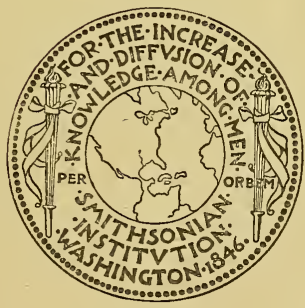

Washington

Government Printing Office 



\title{
THE TYPE-SPECIES OF THE NORTH AMERICAN GENERA
} OF DIPTERA.

\author{
By D. W. Coquillett, \\ Custodian of Diptera, C. S. National Museum.
}

The great importance of knowing definitely what species is the type of any given genus is now recognized by practically erery worker in the field of biology. For several years past the writer has been engaged in ascertaining the types of the genera of Diptera reported as occurring in North and Middle America, and the present paper gives the results of these labors. The rules adopted by the International Zoological Congress, as amended at the 1907 (Boston) meeting and the later decisions, published in Science for October 29, 1909, have been followed in all cases.

The following rules or articles more especially concern us in the present work:

Article 2. "The scientitic designation of animals is uninominal for subgenera and all higher groups." A genus or subgenus, to which no species was originally referred by name, dates from its earliest published description or figure.

Article 3 specifies that the scientific names of animals must be in Latin or, at least, must be latinized. This excludes certain works where only French or other vernacular names are employed, such as Duméril's Exposition d'une Méthode Naturelle, published in 1801; his Considérations Générales, 1823; Schinz's Das Thierreich, 1823, and Latreille's Familles Naturelles du Règne Animal, 182̌́.

Article 19. "The original orthography of a name is to be preserved unless an error of transcription, a lapsus calami, or a typographical error is evident." The so-called emended names are to be regarded only as misspelled names, and as such have no permanent place in the nomenclature.

Article 25. The well known law of priority. This article holds, among other things, that a new generic name unaccompanied by either a description or a figure is valid if the name of one or more described species is mentioned as pertaining to it.

Proceedinas U. S. National Museum, Vol. 37-No. 1719. 
Article 26. Zoological nomenclature began with the year 1758 .

Article 27. "The law of priority obtains, and consequently the oldest available name is to he retained." It further asserts that a name, whether generic, subgeneric, or specific, founded on any part of an animal, or of any of its early stages, is valid.

Article 30. The type of any polytypical genus is that one of the original species first designated as such type. An exception occurs in those cases where the genus originally contained only two species, neither of which had been designated the type by the founder of the genus, nor by any subsequent writer, and an author later takes one of these species as the type of a second genus, he thereby definitely makes the remaining species the type of the old genus.

Article 34. "A generic name is to be rejected as a homonym when it has previously been used for some other genus of animals." Unless the two names are identical, letter for letter, they are not homonyms.

The following list contains all of the genera of Diptera known to the writer as having been reported from North and Middle America up to January 1, 1909, together with their type-species and synonymy. A few genera, such as those to which no species has ever been assigned, and sone others, founded on foreign species, are omitted in this list; but as nearly all in this class are synonyms of older generic names their onission in no way affects the status of the valid names adopted in the present list.

In the cases of those neglected, polytypical genera whose types have not heretofore been designated, and which contain anong their original species one belonging to an older genus, such a species has been selected as the type, thus, as far as possible, sinking this class of names into the synonymy. In this way, fewer changes have resulted among the current names than would have been the case had the opposite course been pursued. In selecting the types of polytypical genera now in current use, it has been my constant aim to select such a species as would result in the maintenance of the present status of the genus. The recommendations appended to Article 30 of the International Code, as amended at the 1907 (Boston) meeting of the International Zoological Congress, have heen essentially followed.

The synonymy of the European species and, in most cases, the limits of the genera, are those given in the monumental Katalog der Paläarktischen Dipteren, by Kertesz, Bezzi, Stein, and Becker, a most admirable work, in four octaro volumes. Some idea of the magnitude of the labors of its authors in unraveling and recording the greatly involved synonymy may be gleaned from a few facts relating to a single species, Tachinu vulgaris Fallen. This species has been redescribed and renamed no less than two-hundred and fifty-seren times! On this one species alone Robineau-Desvoidy established the 
almost incredible number of two-hundred and forty-five new species (so-called), which he distributed into five (imaginary) new genera! And this is only one sample from among the many cases. ${ }^{a}$

In one instance I have differed with the authors of the Katalog in the generic assignment of one species, Tipula culiciformis De Geer, which these authors place in the genus Corethra, but which there is every reason for believing belongs to the preceding genus, Mochlonyx. The breeding of this and related species by Meinert, his reference of the present species to Mochlonyx, and the confirmation of such reference by von Röder are set forth in my article in the Canadian Entomologist for July, 1903. I recently wrote to Dr. J. C. H. de Meijere, the leading dipterologist of Holland, for an expression of his opinion on the subject, and under date of September 28, 1908, he wrote me, entirely corroborating this reference, saying, "As to Corethra culiciformis De Geer, it is my opinion that Meinert has demonstrated with certainty that this gnat is a Mochlonyx." The reference of this species in the Katalog is therefore clearly erroneous.

In most cases subgeneric names have been thrown into the synonymy.

In the accompanying list, the writer has personally examined the original references with the exception of a small number, the data of which were obtained at second hand, such genera being indicated by an asterisk (*). The references in regard to the genera founded by Clark in his Essay on Bots (1815) were kindly communicated to me by Mr. E. E. Austen, of the British Museum, while those from Berthold's Natürliche Familien des Thierreichs (1827) were furnished to me by Mr. E. T. Cresson, jr., of the Academy of Natural Sciences of Philadelphia, and to both of these gentlemen my thanks are due.

Names of genera which are considered valid by the best authorities are printed in black-face type, but several of those so indicated are to be considered as such only from want of authoritative knowledge on the subject. Synonyms and homonyms are printed in italics. ${ }^{b}$

Although the preparation of this paper has extended over many years, and every opportunity has been taken to ascertain the earliest founding of each genus and the first designation of a type-species for the polytypical genera, still it is possible, or even probable, that some earlier records have been overlooked, and the writer will be under obligations to anybody informing him of such previous records.

$a$ The Katalog appears to be very complete, except that it omits most of the genera of the Anthomyiidie and Acalypteræ founded by Lioy in the Atti Instituto Veneto, ser. 3, vol. 9, 1863 and 1864. The greater number of the genera founded by Meigen in his Nouvelle Classification des Mouches à denx ailes (Diptera L.), 1800, are also omitted; there is a reprint of this paper, with comments and synonymy, by Mr. Friedrich Hendel in the Verh. k. k. Zool.-Bot. Ges. Wien, vol. 58, pages 43 to 69,1908 , which I have made use of in the present paper.

$b$ The generic names are arranged alphabetically, for easy reference. An index to the species is appended to this paper. 
$\sqrt{\text { Ablabesmyia Johannsen, Bull. 86, N. Y. State Mus., p. 125, 1905. } 24 \text { species. }}$ Type, Tipula monilis LrNnzus, the sixth species, by present designation.

$\checkmark$ Ablautus LoEw, Berliner Ent. Zeitschr., vol. 10, p. 37, 1866. 1 species. Type, Ablautus trifarius LoEw.

Acanthina Wiedemann, Auss. Zweifl. Ins., vol. 2, p. 50, 1830. 3 species. Type, Clitellaria elongata Wredemann, the third species, by designation of Brauer, Denkschr. Kais. Akad. Wiss. Wien, vol. 44, 1882, p. 86. Not Acanthina Fischer, 1806. Equals Artemita WALKER, 1854.

- Acunthinomyir Hunter, Trans. Amer. Ent. Soc., vol. 27, p. 129, 1900. Change of name for Acanthina Wiedeniann, 1830, not of Fischer, 1806. Type, Clitellaria elongata Wiedemann. Equals Artemita Walker, 1854.

$\checkmark$ Acanthocnema Becker, Berliner Ent. Zeitschr., vol. 39, p. 136, 1894. 3 species. Type, Cordilura nigrimana Zettenstent, by original designation.

Acanthomera Wiedemann, Dipt. Exot., p. 60, 1821. 1 species. Type, Acanthomera picta Wiedemann. Equals Pantophthalmus Trunberg, 1819.

$\checkmark$ Acaulona Wulp, Biol. Centr.-Amer., Dipt., vol. 2, p. 4, April, 1888. 1 species. Type, Acaulona costata Wulp. Syn., Euacaulona Townsend, 1908; Euomogenia Townsend, 1908.

$\checkmark$ Acemya Desvorny, Essai Myod., p. 202, 1830. 1 species (as 3). Type, Tachina acuticornis Meigen. Syn., Agculocera Macquart, 1855.

- Achætomus Coquillett, Can. Ent., vol. 39, p. 75, MIarch, 1907. 1 species. Type, Achrtomus pilosus Coquillett.

- Achetoneura Brauer and Bergenstamm, Denkschr. Kais. Akad. Wiss. Wien, vol. 58, p. 333, 1891. 5 species. Type Musicera frenchii Willistox, the first species (as hesperus, new species), by present designation. Equals Frontina Melgen, 1838.

$\checkmark$ Achalcus Loew, Neue Beitr., vol. 5, p. 30, 1857. 2 species. Type, Porphyrops Aavicollis MIEIGEN, the first species, by present designation.

$\checkmark$ Acicephala Coquillett, Journ. N. Y. Ent. Soc., vol. 6, p. 163, 1898. 2 species. Type, Acicephala polita Coqunlett, the first species, by original designation.

$\checkmark$ Acidia Desvordy, Essai Myorl., p. 720, 1830. 2 species. Type, Tephritis cognata Wiedemann, the recond species, by designation of Rondani, Bull. Soc. Ent. Ital., vol. 2, 1870, p. 10. Syn., Euleia WaLker, 1836; Elidesmia Rondani, 1856; Myoleja Rondani, 1856; Prionimera Rondani, 1861; Philophylla Rondani, 1870.

$\checkmark$ Acidigona Loew, Monogr. Dipt. N. Amer., vol. 3, p. 285, 1873. 1 species. Type, Trypeta melanura LoEw.

$\checkmark$ Aciura Desvoiny, Essai Myod., p. 773, 1830. 2 species. Type, Aciura femoralis Desvolny, the first species, by designation of Rondani, Dipt. Ital. Prodr., vol. 1, 1856, p. 113.

Acnemia Winnertz, Verh. Zool.-Bot. Ges. Wien, vol. 13, p. 798, 1863. 3 species. Type, Leia nitidicollis MeIgex, the first species, by designation of JoHANxsen in Wytsman's Gen. Ins., Dipt., Mycet., 1909, p. 63.

$\checkmark$ Acontistoptera Brues, Amer. Nat., vol. 36, p. 373, May, 1902. 1 species. Type, Acontistoptera melanderi Brues.

$\checkmark$ Acreotrichus Macquart, Dipt. Exot., Suppl. 4, p. 121, 1850. 2 species. Type, Acreotrichus gibbicornis МАCQUART, the first species, by present designation.

Acrocera Meigen, Illiger's Mag., vol. 2, p. 266, 1803. 1 species. Type, Syrphus globulus Panzer. Syn., I'aracrocera Miк, 1886.

$\mathcal{A}$ Acrochæta Wiedemann, Auss. Zweifl. Ins., vol. 2, p. 42, 1830. 1 species. Type, Acrochrta fasciata WiedeMANN.

$\checkmark$ Acrocholidia Kolenat1, Wien. Ent. Monatschr., vol, 1, p. 62, 1857. 2 species. Type, Acrocholidia bechsteinii Kolenati, the first species, by present designation. Equals Nycteribia Latreille, 1796.

Acrochordonodes Bigot, Bull. Soc. Ent. France for 1878, p. 97, 1878. 1 species. Type, Acrochordonodes vittatus Bigot. Equals Senogaster Macquart, 1834. 
Acroglossa Williston, in Scudder's Butt. N. Engl., vol. 3, p. 1916, 1889.1 species. Type, Acroglossa hesperilurum Wrulrston. Equals Spallanzania Desvordy, 1830.

Acrometopia Schiner, Wien. Ent. Monatschr., vol. 6, p. 434, 1862. Change of name for Oxyrhina MeIgex as erroneously identified by ZetTerstedt. 2 species. Type, Oxyrhina wahlbergi ZETTERsTEDT, the first species, by original designation of ZETTERSTEDT.

Acrometopia Lror, Atti Inst. Veneto, ser. 3, vol. 9, p. 1088, 1864.1 species. Type, Sepsis comuta Meigen. Equals Sepsis Fallen, 1810.

Acromyia Latreille, Gen. Crust. et Ins., vol. 4, p. 305, 1809. 1 species. Type, Asilus muscarius FABRicius (as Acromyia asiliformis. In the article entitled "Hybos," in the Nouv. Dict. Hist. Nat., $2 \mathrm{~d}$ ed., 1818, Latreille wrote that he had received from Bonelli a specimen of this genus under the generic name of Acromya). Syn., Syneches WaLker, 1852; Pterospitus Rondani, 1856.

Acronacantha Wulp, Biol. Centr.-Amer., Dipt., vol. 2, p. 243, 1891.1 species. Type, Acronacantha nubilipennis WuLP.

Acronarista Townsend, Smiths. Misc. Coll., vol. 51, p. 85, 1908. 1 species. Type, Acronarista mirabilis Townsend.

Acrosticta Loew, Berliner Ent. Zeitschr., vol. 11, p. 293, 1868. 2 species. Type, Acrosticta scrobiculata Loww, the first species, by present designation.

Acrotænia Loww, Monogr. Dipt. N. Amer., vol. 3, p. 274, 1873. 2 species. Type, Trypeta testudinea Losw, the first species, by present designation.

Acrotoxa Loww, Monogr. Dipt. N. Amer., vol. 3, p. 227, 1873. 11 species. Type, Dacus fraterculus Wiedemans, the second species, by designation of Bezzr, Boll. Lab. Zool. Portici, vol. 3, p. 280, 1909. Equals Anastrepha ScHrver, 1868.

Actia Desvordy, Essai Myod., p. 85, 1830. 1 species (as two new ones). Type, Actia cingulata Desvordy. Syn., Osmra Desvordy, 1830; Thryptocera Macquart, 1834; Elfia Desvorny, 1850; Herbstia Desvordy, 1851; Peribra Desvordy, 1863; Gymnophtalma Lroy, 1864; Gymnopareia Brauer and Bergenstamu, 1889.

Actina Meigen, Klass. Beschr. Zweifl. Ins., p. 116, 1804. 3 species. Type, Actina chalybea Meigen (as Beris nitens Mergen), the first species, by designation of RonDANI, Arch. Zool., vol. 3, 1864, p. 87.

Actinoptera Rondani, Bull. Soc. Ent. Ital., vol. 3, p. 164, 1871. 3 species. Type, Trypeta æstiva MeIGen, the second species, by present designation. Equals Trupanea Schrank, 1795.

Actora Mergen, Syst. Beschr. Zweifl. Ins., vol. 5, p. 403, 1826. 1 species. Type, Actora xsturm Meigen. Equals Helcomyza Curtis, 1825.

Adelinia Costa, Il Giamb. Vico, vol. 2, p. 447, 1857.* 1 species. Type, Adelinia italica Costa. Equals Asindulum LatremLle, 1805.

Adenia Desvordr, Hist. Nat. Dipt., vol. 1. p. 1041, 1863. 1 species (as 10). Type, Tachina simulans MeIGeN. Equals Exorista MeIGeN, 1803.

Adia Desvordy, Essai Myod., p. 558, 1830. 1 species. Type, Adia oralis Desvoliy. Equals Pegomya Desvoiny, 1830.

Adiplosis FELT, 23d Rep. State Ent. N. Y., p. 405,.1908. 1 species. Type, Cecidomyia toricodendri FELT.

$\checkmark$ Admontia Brauer and Bergenstam, Denkschr. Kais. Akad. Wiss. Wien, vol. 56, p. 104, 1889. 1 species. Type, Degeeria amica MeIgen (as podomyia, new species). Equals Hyperecteina ScHiner, 1861.

Adoxomyia Kertesz, Ann. Mus. Nat. Hungarica, vol. 5, p. 499, 1907. New name for Clitellaria Schiner, not of Meigen. 2 species. Type, Clitellaria dahlii Mrigen, the first species, by designation of Bezzi, Wien. Ent. Zeit., vol. 17, p. 75, 1908.

Ecothea Halmay, Ann. Nat. Hist., vol. 2, p. 187, November, 1838. 1 species. Type, Heleomyza fenestralis FALLeN.

-Aëdeomyia Theobald, Journ. Trop. Med., vol. 4, p. 235, July 15, 1901 . No species. In Monogr. Culic., vol. 2, p. 219, Nov. 23, 1901, 1 species. Type, Aëdes squamipenns Arribalzaga. 
$\sqrt{ }$ Aëdes Wiedenann, in Meigen's Syst. Beschr. Zweifl. Ins., vol. 1, p. 13, 1818. 1 species. Type, Aëdes cinereus WIEDEMAxN.

Enigmatias Mieinert, Ent. MIedd., vol. 2, p. 213, 1890. 1 species. Type, Enigmatias blattoides MeINert.

$\checkmark$ Agatachys Meigen, Syst. Beschr. Zweifl. Ins., vol. 6, p. 343, 1830. 1 species. Type, Thechydromia celeripes Meigen. Syn., Stilpon Loew, 1859.

Agathomyia Terrall, Brit. Flies, vol. 8, p. 30, 1901. 2 species. Type, Caklomyza antennata ZETTERSTEDT, the first species, by present designation.

レ Agathon Rö̀er, Wien. Ent. Zeit., vol. 9, p. 230, 1890. 1 species. Type, Agathon elegantula RöDER. Equals Bibiocephala Osten SAckex, 1874.

$\checkmark$ Agculocera Macquart, Ann. Soc. Ent. France for 1855, p. 24, 185.5. 1 species. Type, Tarhina acuticornis Meigen (as nigra, new species). Equals Acemya DesroIDr, 1830.

$\checkmark$ Agelanius Rondani, Arch. Zool., vol. 3, p. 79, 1864. 2 species. Type, Agelanius meridiamus RoNDANi, the first species, by present designation. Equals Tabanus LiNx.ets, 1758.

$\checkmark$ Agnotomyia Wrulistox, Ent. Amer., vol. 2, p. 106, 1886. 1 species. Type, Slygia elongata SAX. Equals Dialysis WALKer, 1850.

$\checkmark \quad$ Agria Desvoldy, Essai Myod., p. 376, 1830. 1 species (as 6). Type, Musca affinis Fallex. Equals Sarcophaga Meigen, 1826.

$\checkmark$ Agrobia Lroy, Atti Inst. Teneto, ser. 3, vol. 9, p. 1313; 1864. 1 species. Type, Agromyza pectinata Meigex. Equals Agromyza FAllex, 1810.

Agromyza Fallen, Spec. Ent. Meth. Exh., p. 21, 1810. No species. In his Agromyzides Sveciæe, pp. 3 to 7, 1823, 14 species (1 as a variety). Type, Agromyza ambigia FAlLEN (described as a variety of the first species), by designation of Wrestwood, Intr., rol. 2, Synops., p. 151, 1840 (as nigripes Meigex). Syn., Phytubia Liox, 1864; Phyllophila Lıox, 1864; Agrobia Lıoy, 1864; Redia Lroy, 1864; Agrophila Lioy, 1864; Anisonerra Lior, 1864.

$\checkmark$ Agrmphila Liox, Atti Inst. Veneto, ser. 3, vol. 9, p. 1314, 1864. 4 species. Type, Agromyza pxilis Meigev, the first species, by present designation. Equals Agromyza FALLEN, 1810.

Akronia Hine, Ohio Nat., rol. 1, p. 113, 1901. 1 species. Type, Akronia frontosa Hine.

$\checkmark$ Alasion Rondani, Dipt. Ital. Prodr., vol. 2, p. 14, 1857. Change of name for Apogon Rondani, 1856, preoceupied. Type, Ceratopogon flaripes Meigex. Equals Helea Meigen, 1800.

$\checkmark$ Albinia Desvorny, Essai Myod., p. 209, 1830. 1 species. Type, Albinia buccalis Desvordy. Equals Macquartia Desvordy, 1830.

$\checkmark$ Alcephagus Ginıerthal, Ent. Zeit. Stettin, vol. 6, p. 152, 1845. Change of name for Ornithobia Meigen, 1830, on the ground that the latter name is not appropriate. Type, Pediculus cervi Linx.eus. Equals Lipoptena Niтzsch, 1818.

Aldrichia Coquillett, Trans. Amer. Ent. Soc., vol. 21, p. 93, 1894. 1 species. Type, Aldrichia elimmani Conulllett.

Alina Desroidy, Hist. Nat. Dipt., vol. 2, p. 689, 1863. 3 species (as 4). Type, Alina pratensis Desvordy, the second species, by present designation. Equals Morellia Desvoidy, 1830.

Allocotus Loew, Berliner Ent. Zeitschr., vol. 16, p. 258, 1872. 1 species. Type, Allocotus edwardsii Loew. Not Allocotus Ma Yr, 1864. Equals Paracosmus Osten SACKex, 1877.

$\checkmark$ Allodia Winnertz, Verh. Zool.-Bot. Ges. Wien, vol. 13, p. 826, 1863. 5 species. Type, Mycetophila lugens Wiedemans, the fourth species, by designation of Jolinnssen in Wytsman's Gen. Ins., Dipt., Mycet., 1909, p. 104. Syn. Brachycampta Winvertz, 1863. 
Allooneurus Мrk, Jahresb. k. k. Akad. Gymnas., p. 8, 1878. 1 species. Type, Irusca lacustris Scopoli. Equals Orthoceratium Scopoli, 1803.

Allognosta Osten SAcken, Berliner Ent. Zeitschr., vol. 27, p. 297, 1883. Change of name for Metoponia Loew, not of Macetant. 3 species. Type, Beris fuscitarsis SAY, the third species, by present designation.

Allograpta Ostex SACKen, Bull. Buffalo Soc. Nat. Hist., vol.3, p. 49, 1876. 1 species. Type, Scæva obliqua SAY.

Alloneura Rondani, Dipt. Ital. Prodr., vol. 1, p. 140. 1856. 1 species. Type, Pipunculus minimus Becker (as flaripes Mergen). Equals Dorilas Meigen, 1800.

Allophorocera Hendel, Verh. Zool.-Bot. Ges. Wien, vol. 51, p. 203, 1901.1 species. Type,Dexodes auripila Brauer and Bergenstamu. Equals Lydella DesYOIDY, 1830.

Allophyla Loew, Zeitschr. Ent. Breslau, vol. 13, p. 43, 1862. 1 speeies. Type, Heleomyza atricornis MeIGEN.

$\checkmark$ Allotrichoma Becker, Berliner Ent. Zeitsehr., vol. 41, p. 121, 1896. 4 species. Type, Hecamede lateralis Loww, the first species, by original designation.

$\checkmark$ Alophora Desroidx, Essai Myod., p. 293, 1830. 1 species (as 4). Type, Syrphus hemipterus Fabricius. Equals Phasia Latreille, 1805.

Alticomerus Rondani, Dipt. Ital. Prodr., vol. 1, p. 121, 1856. 1 species. Type, Milichia maculata Meigen (as trinotatus, new species). Equals Odinia Desvordy, 1830.

$\boldsymbol{V}$ Amalopis Haliday, in Walker's Ins. Britt., Dipt., vol. 3, p. xv, 1856. 1 species. Type, Limnobia occulta Meigen. Equals Tricyphona Zetterstedt, 1837.

$\checkmark$ Amedea Desvoidy, Essai Myod., p. 207, 1830. 1 species. Type, Amedea scutellaris Desvordy. Equals Macquartia Desvordy, 1830.

$\checkmark$ Amedoric Brauer and Bergenstamm, Denkschr. Kais. Akad. Wiss. Wien, vol. 56, p. 106, 1889. 1 species. Type, Tachina luctuosa Meigen (as medorina Schiner). Equals Medina Desvordy, 1830.

$\checkmark$ Amesia Desvoldy, Hist. Nat. Dipt., vol. 2, p. 363, 1863. 1 species. Type, Tachina disjuncta Wiedemann (as variabilis, new species). Equals Microphthalma Macquart, 1843.

$\checkmark$ Amina Desvordy, Essai Myod., p. 626, 1830. 1 species. Type, Amina parisiensis Desvorny. Equals Scopeuma Meigen, 1800.

Aminta Desvordy, Essai Myod., p. 569, 1830. 5 species. Type, Aminta ludibunda Desvordy, the first species, by present designation. Equals Fannia Desrordy, 1830,

$\checkmark$ Amiota Loew, Berliner Ent. Zeitschr., vol. 6, p. 229, May, 1862. 2 species. Type, Amiota leucostoma Loew, the second species, by present designation. Syn., Phortica Schiner, Dec., 1862.

$\checkmark$ Ammobates Stannius, Isis von Oken for 1831 , p. 33, 1831. 3 species. Type, Ammobates notatus Stannius, the second species, designated by Rondani, Dipt. Ital. Prodr., vol. 1, 1856, p. 143. Not Ammobates Latreille, 1809. Equals Tachytrechus Haliday, 1851.

$\checkmark$ Amobia Desvordy, Essai Myod., p. 96, 1830. 1 species. Type, Amobia conica Desvoidy. Syn., Macronichia Rondani, 1859; Moschusa Desvoidy, 1863; Theone DesvoIDr, 1863; Trixoclista Townsend, 1892.

Amphicnephes Loew, Monogr. Dipt. N. Amer., vol. 3, p. 83, 1873.1 species. Type, Trypeta pulla Wiedemann (as pertusus, new species).

Amphicosmus Coquilletr, West Amer. Sci., vol. 7, p. 219, 1891. 1 species. Type, Amphicosmus elegans CoQUILLeTT.

- Amphinome Mrigex, Nouv. Class. Mouch., p. 15, 1800.* No species. Limonia Meigen, 1803, is a change of name. Type, Tipula tripunctata FABricius. Syn., Limonia Metgen, 181)3; Limnobia Meigen, 1818; Unomyia Meigen, 1818; Limnomyza RonDANI, 1856. 
Ampycophora WAflbers, Öfv. Kongl. Vet. Akad. Förh., vol. 4, p. 261, 1847. 1 species. Type, Aulacigaster mufitursis Macruart (as tarsata, new species). Equals Aulacigaster MACQUART, 1835.

$\checkmark$ Ampyx Walker, List Dipt. Ins. Brit. Mus., vol. 7, p. 564, 1855. 1 species. Type, Asilus distendens Wiedemann (as varipennis, new species). Syn., Doryclus J ennicke, 1867.

$\checkmark$ Amsteinia An Steıx, Jahresb. Nat. Ges. Graubünden., ser. 2, vol. 3, p. 99, 1858. 1 species. Type, Crameria astroidea Destoldy (as punetipennis, new species). Equals Trixa Meigex, 1824.

$\checkmark$ Amyclxa Dessoldy, Hist. Nat. Dipt., vol. 2, p. 404, 1863. 1 species. Type, Amyclaca serva Desvoidy. Equals Phorostoma Desvoni, 1830.

Anacampta Loew, Zeitsch. Ges. Naturwiss; vol. 32, p. 7, 1868. 4 species. Type, Musca urticx Linnsus, the first species, by designation of Loww, Monogr. Dipt. N. Amer., vol. 3, 1873, p. 58. Equals Ceroxys Macrevant, 1835.

$\checkmark$ Anaclinia Winsertz, Verh. Zool.-Bot. Ges. Wien, vol. 13, p. 770, 1863. 1 species. Type, Mycetophila nemoralis Meigen.

Analcocerus Loew, Verh. Zool.-Bot. Gies. Wien, vol. 5, p. 140, 1855. 1 species. Type, Analcocerus atriceps LoEw.

$\checkmark$ Anarmostus Loew, Dipt.-Fauna Südafrika, p. 142, 1860. 1 species. Type, Asilus iopterus WIEDEMANN.

$\checkmark$ Anasimyia Schiner, Cat. Dipt. Europre, p. 108, 1864. 2 species. Type, Muscatransfuga Linn.eus, the first species, by present designation. Equals Helophilus Meigen, 1822.

$\checkmark$ Anastochus Ostex SAckev, West. Dipt., p. 251, 1877. 1 species. Type, Bombylius nitidulus FABricios (as barbatus, new species).

$\checkmark$ Anastrepha Schixer, Reise Noraru, Zool., vol. 2, Dipt., p. 263, 1868. 6 species. Type Dacus serpentinus Wiedenann, the first species, by original designation. Syn., Acrotora Loew, 1873.

$\checkmark$ Anatopynia Johandsen, Bull. 86, N. Y. State Mus., p. 135, 1905.1 species. Type, Tanypus plumipes Fries.

Ancylogaster B1Got, Bull. Soc. Ent. France for 1884 , p. 69, 1884. 1 species. Type, Hemyda aurata Desroidy (as armatus, new species). Equals Hemyda Desroidy, 1830. Audrenomya Rondan1, Nuovi Ann. Sci. Nat. (Bologna), ser. 3, vol. 2, p. 189, 1850. 1 species. Type, lihynchocephalus caucasirus Flscher (as Nemestrina albofasciata Wredenans). Equals Rhynchocephalus Fischer, 1806.

Andrenosoma Rondaxi, Dipt. Ital. Prollr., vol. 1, p. 160, 1856. 1 species. Type, Asilus ater Linneus. Syn., Elxotoma Costa, 1863.

- Anepsiomyia Bezz1, Zeitsch. Hym. Dipt., vol. 2, p. 192, 1902. Change of name for Anepsius Loew, 1857, not of LeConte, 1852. Type, Porphyrops fluvicutris Melciex. Syn., Anepsins Loww, preoceupied.

$\checkmark$ Anepsins Loww, Nene Beitr., vol. 5, p. 45, 1857. 1 species. Type, Poryluyrops flavirentris Meigen. Not Anepsins LeConte, 1852. Equals Anepsiomyia Bezzi, 1902.

- Anetia Desvolny, Hist. Nat. Dipt., vol. 1, p. 868, 1863. 1 species. Type, Tuchina nigripes Falden (as occlusa, new species). Equals Lydella Desvowr, 1830.

- Anevrina Lioy, Atti Inst. Venetu, 3i ser., vol. 10, 1. 77, 1864. 2 species. Type, Phora caliginosa Menciex, the first species, by present designation. (Equals Phora of authors, not of LATREILLE.)

- Angiorhina Braler and Bergenstamu, Denkschr. Kais. Akad. Wiss. Wien, vol. 56, p. 163, 1889.1 species. Type, Tachina crudelis Wiedenanx.

Angleariu Carlier, Ann. Soc. Ent. France for 1835, p. 659, 1835. 1 species. Type, Anglearia antemata Carlier. Equals Perithinus Halmar, 1831.

Anicia Desvondy, Hist. Nat. Dipt., vol. 2, p. 99, 1863. 1 species (as 4). Type, Anicia sabulosu Desvoidy. Equals Metopia Meigen, 1803. 
Anisia Wulp, Biol. Centr.-Amer., Dipt., vol. 2, p. 186, August, 1890. 41 species. Type, Anisia inflexa Wulp, the first species, by present designation.

Anisomera Wiedemann, in Meigen's Syst. Beschr. Zweifl. Ins., vol. 1, p. 210, 1818. 1 species. Type, Anisomera obscura Wiedemanx. Equals Hexatoma Latreirle, 1809 .

- Anisonevra Lioy, Atti Inst. Veneto, ser. 3, vol. 9, p. 1314, 1864. 1 species. Type, Agromyza lacteipennis Fallen? Equals Agromyza Fallen, 1810.

- Anisopogon Loew, Berliner Ent. Zeitschr., vol 18, p. 377, 1874. Change of name for Heteropogon Loew, 1847, previously used for a genus of plants. Type, Dasypogon manicatus MeIGen. Equals Heteropogon Loew, 1847.

- Anisopus Meigen, Illiger's Mag., vol. 2, p. 26t, 1803. No species. In his Klass. Beschr. Zweifl. Ins., p. 103, 1804, 2 species. Type, Tipula fuscata FABricius (as fuscus, new species), the first species, by present designation. Equals Sylvicolæ HARRIS, 1776.

Anisotamia Macquart, Dipt. Exot., vol. 2, pt. 1, p. 81, 1840. 2 species. Type, Anisotamia ruficornis MACQUART, the first species, by present designation.

- Anomaloptera Liox, Atti Inst. Veneto, ser. 3, vol. 9, p. 218, 1864. 1 species. Type, Tipula nigra Linneus. Equals Tipula Linnæus, 1758.

- Anopheles Meigen, Syst. Beschr. Zweifl. Ins., vol. 1, p. 10, 1818. 2 species. Type, Culex bifurcatus Linsius, the first species, by designation of Cuntis, Brit. Ent., 1828, p. 210. Syn., Coelodiazesis Dyar and KNAB, April 15, 1906; Myzorhynchella TheовaLd, Jan., 1907.

Anoplomerus Rondani, Dipt. Ital. Prodr., vol. 1, p. 141, 1856. 1 species. Type, Musca notata Fabricius. Not Anoplomerus Latreille, 1844. Equals Scellus Loew, 1857.

$\checkmark$ Anoplopus Rondani, Dipt. Ital. Prodr., vol. 2, p. 14, 1857. Change of name for Anoplomerus Rondani, 1856, not Latreille, 1844. Type, Ifusca notata Fabricius. Equals Scellus LoEw, 1857 .

Anorostoma Loew, Zeitschr. Ent. Breslau, vol. 13, p. 47, 1862 . No species. In the Berliner Ent. Zeitschr., vol. 6, 1862, p. 223, 1 species. Type, Anorostoma marginata Loew.

$\checkmark$ Anorthus LoEw, Ent. Zeit. Stettin, vol. 11, p. 117, 1850. 1 species. Type, Hydrophorus jaculus Falden. Equals Medetera Fischer, 1819.

Anoxycampta Bigot, Bull. Soc. Ent. France for 1880, p. 150; 1880. 1 species. Type, Anoxycampta hirta Bigot. Equals Phryxe Dezvoidy, 1830.

Anthaliı Zetrerstent, Ins. Lapp., p. 538, 1838. 3 species. Type, Anthalia gyllenhali Zetterstedt, the first species, by designation of Coquillett, Proc. Ent. Soc. Washington, vol. 5, 1903, p. 246. Equals Euthyneura MACQUart, 1836.

$\checkmark$ Anthoica Rondani, Dipt. Ital. Prodr., vol. 4, p. 8, 1861. Change of name for Myobia Desvoldy, 1830, preoccupied. Type, Tachina inanis Fallen. Equals Leskia DesvoIDY, 1830

Anthomyia Meigen, Illiger's Mag., vol. 2, p. 281, 1803. 2 species. Type, Musca pluvialis LINNeus, the second species, by designation of Westwood, Intr., vol. 2, Synops., 1840, p. 143.

Anthomyza Fallen, Spec. Ent. Meth. Exh., p. 20, 1810. No species. In his Agromyzides Sveciæ, pp. 7 and 8, 1823, 4 species. Type, Anthomyza gracilis Fallen, the third species, by designation of Westwood, Intr., vol. 2, Synops., 1840, p. 152. Syn., Leptomyza Macquart, 1835; Anthophilina Zetterstedt, 1837; Psilosoma Lior; 1864

$\checkmark$ Anthophilina Zettenstedt, Isis von Oken for 1837, p. 55, 1837. 1 species (and 3 manuscript names). Type, Anthomyza gracilis Fallen. Equals Anthomyza Fallen, 1810.

$\checkmark$ Anthracomya Rondani, Dipt. Ital. Prodr., vol. 1, p. 87, 1856. 1 species. Type, Musca melanoptera Fallen (as geneji, new species). Equals Morinia Desvoidy, 1830. 
Anthracophaga Loew, Zeitschr. Ent. Breslau, vol. 15, p. 15, 1866.* 3 species. Type, Muscre strigula FABricius, the first species, by present designation.

Anthrax Scopolr, Ent. Carn., p. 358, 1763. 1 species. Type, Iusca anthrax Schrank (as morio Linnfeus). Syn., Spogostylum Macrecart, 1840; Argyramolar Schiser, 1860; Coquillettia Willstox, 1896. (Anthrax of authors equals Tilla Lros.)

- Anticheta Halidax, Ann. Nat. Hist., vol. 2, p. 187, November, 1838. 1 species. Type, Sciomyza analis MIEIGEN (as Titanocera vittate HALIDAY).

- Antiopa Meigex, Nouv. Class. Mouch., p. 32, 1800** No species. Chrysotoxum

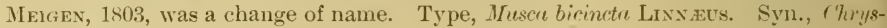
otoxum MEIgen, 1803.

$\checkmark$ Intlemon Loew, Syst. Beschr. Zweifl. Ins., vol. 2, p. 30, 1871. 1 species. Type, Asinclulum halidayi Loew. Equals Asindulum Latreille, 1805.

$\checkmark$ Antocha Osten SAckex, Proc. Acal. Nat. Sci. Phila. for 18.59, p. 219, 1860. 1 species (as 2). Type, Antocha saxicola Ostex Sackex. Syn., Orimurgulu Mrk, 1883.

$\checkmark$ Amppenus Prilippi, Verh. Zool.-Bot. Ges. Wien, vol. 15, 1). 702, 186.5. 2 species. Type, Anypemus obscums Prilıpr, the second species, by present designation. Equal: Apiocera Westwoon, 1835 .

$\checkmark$ Aochletus Osten Sacken, Biol. Centr.-Amer., Dipt., vol. 1, p. 38, 1886.1 species. Type, Aochletus cinctus OSTEN SAc KEx.

- Apachemyin Towrsexn, Smiths. Misc. Coll., vol. 51, p. 75, 1908. 1 species. Type, Demoticus pallidus Conulletet. Equals Demoticus Macquart, 18.54.

2. Apatolestes Willistos, Ent. Amer., vol. 1, p. 12, 1885. 1 species. Type, Apatolestes comaster WiLLISTON.

- Apeilesis Macruart, Dipt. Exot., Suppl. 1, p. 8, 18t6. 1 species. Type, Apeilesis cinerer Macquart. Equals Dolichopeza Curtis, 1825.

$\checkmark$ Apelleia Bellardi, Saggio Ditt. Ness., Append., p. 17, 1862. 1 species. Type, Apelleiu rituta BELLARDI.

- Aphantotimus Wheeler, Psyche, wol. 5, p. 375, 1890. 2 species. Type, Aphanto-

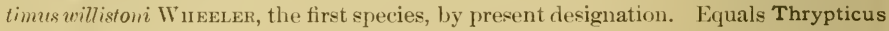
GERstacker, 1864.

$\checkmark$ Aphestia Schner, Verh. Zool.-Bot. Ges. Wien, rol. 16, p. 673, 1866. 1 species. Type, Aphestia lrasiliensis Sch Iner.

- Aphiochatr Bruw, Trans. Amer. Ent. Sric., vol. 29, p. 337, 1903. 24 species. Type, Phora nigriceps Loew, the tenth species, by designation of Brues, Gen. Ins., Dipt., Phoridre, 1906, p. 9. Equals Megaselia Rondani, 1856.

Aphœbantus LoEw, Berliner Ent. Zeitschr., vol. 16, p. 253, 1872. 1 species. Type, Aphobantus cervinus Loew. Syn., Triodites Osten SAckex, 1877.

- Aphria Desvoidr, Essai Myod., p. 89, 1830. 1 species (as 2). Type, Tachina longirostris Meigen. Syn., Olivieria Meigen, 1838; Rhynchosia MicqLart, 1848.

$\checkmark$ Aphritis Latreille, Hist. Nat. Crust et Ins., vol. 14, p. 358, 1805. 1 species. Type, Musca mutabitis Linneus (as auropubescens, new species). Equals Microdon Meigex, 1803.

- Aphrosylus Haliday, in Walker's Ins. Britt., vol. 1, p. 220, 1851. 2 species. Type, Aphrosylus raptor HaLidar, the first species, by present designation.

- Aphrozeta Perrik, Iem. Acal. Roy. Sci. Lyon, vol. 2, p. 491, 18ti. 2 species. Type, Medeterce riridis Meiren (as semiglanch, new species), the first species, by present designation. Syn., l'arluydrophorus Wneeler, 1896.

- Apinops Conulllett, Rev. Tach. Amer., p. 67, 1897. 1 species. Type, Apinops atra Coruillett.

$\checkmark$ Apiocera IVestwood, Lond. Edinh. Philos. Mag., vol. 6, p. 448, 1835. 2 species. Type, Ipiocera fuscicollis Westwoon, the second species, by present designation. Syn., Tapinocera Macquart, 1838; Pomacera Macquart, 1847; Anypemus Philippi, 1865 .

$\checkmark$ Ipirora Mejgen, Nouv. Class. Mouch., p. 37, 1800* No species. Equals Volucella Geofrnor, 1762. Type, ILusca pellucens Linnets. 
Aplomya Desvordy, Essai Myod., p. 18t, 1830. 2 species. Type, Tachina confinis Faldex (as zonata, new species), the second species, by designation of Desvoidr, Hist. Nat. Dipt., vol. 1, 1863, p. 459. Syn., Hubneria Desroidy, 1847; Cynisca Desvordy, 1863; Ethilla Desvoidy, 1863.

Apocephalus Coquillett, Proc. Ent. Soc. Washington, vol. 4, p. 501, 1901. 1 species. Type, Apocephalus pergandei Convilletr.

Apogon Rondani, Dipt. Ital. Prodr., vol. 1, p. 175, 1856. 1 species. Type, Ceratopogon faripes Meigen (as hortulanus Meigen). Equals Helea Meigen, 1800.

Apomidas Conuillett, Can. Ent., vol. 2t, p. 315, 1892. 1 species. Type, Apomidas trochilus Coquillett.

Aporomya Rondani, Dipt. Ital. Prodr., vol. 3, p. 90, 1859. 1 species. Type, Tachina dubia Fallen. Equals Lypha Desvordy, 1830.

Aporosa Macquart, Dipt. Exot., vol. 1, pt. 1, p. 62, 1838. 2 species. Type, Geranomyia canariensis Bergroth (as maculipennis, new species), the first species, by present designation. Equals Geranomyia HaLIDAY, 1833.

A porotachina Meade, Ent. Monthly Mag., ser. 2, vol. 5, p. 109, 1894. 5 species. Type, Tachina angeline Mergen, the first species, by present designation. Equals Lydella Desvordy, 1830.

Apostrophus LoEw, Schr. Ges. Freunde Nat. Moseow for 1870, p. 58, 1870.* 1 species. Type, Tachina melanura Meigen (as suspecta, new species). Equals Besseria Destoidi, 1830.

Apotomella Dufour, Ann. Soc. Ent. France for 1846, p. 455, 1846.1 species. Type, Aulacigaster rufitarsis Macquart. Equals Aulacigaster Macquart, 1835.

Aptilotus Mıк, Wien. Ent. Zeit., vol. 17, p. 206, 1898. 1 species. Type, Aptilotus paradoxus Mrk.

Aptorthus Aldrich, Kansas Univ. Quart., vol. 2, p. 48, 1893. 4 species. Type, Aptorthus albiciliatus ALDRICH, the first species, by present designation. Equals Mesorhaga Schiner, 1868.

Araba Desvordy, Essai Myod., p. 127, 1830. 3 species (as 10). Type, Musca leucocephala Rossi (equals species 2 to 9 ), by designation of Desvordy, Hist. Nat. Dipt., yol. 2, 1863, p. 83. Equals Metopia Meigen; 1803.

Arabella Desvordy, Hist. Nat. Dipt., vol. 2, p. 88, 1863. 1 species (as 8). Type, Musca leucocephala Rossi. Equals Metopia Meigen, 1803.

$\checkmark$ Archilestes Schiner, Verh. Zool.-Bot. Ges. Wien, vol. 16, p. 672, 1866. 2 species. Type, Dasypogon capnopterus Wiedeuann, the first species, by designation of Schiner, Reise Novara, Zool., vol. 2, Dipt., 1868, p. 168. Not Archilestes Selys, 1862. Equals Archilestris LoEw, 1874.

$\checkmark$ Archilestris LoEw, Berliner Ent. Zeitschr., vol. 18, p. 377, 1874. Change of name for Archilestes Schiner, 1866, not of SelYs, 1862. Type, Dasypogon capnopterus IViedemann. Syn., Archilestes Schiner, 1866, preoccupied.

$\checkmark$ Archytas J Jennicke, Neue Exot. Dipt., p. 392, 1867. 1 species. Type, Musca diaphana Fabricius (as bicolor, new species). Syn., Nemochxta Wulp, 1888; Tachinodes Brauer and Bergenstamm, 1889; Parafabricic Brauer and Bergenstami, 1894. $\checkmark$ Arctobiella Coquillett, Journ. N. Y. Ent. Soc., vol. 10, p. 188, 1902. 1 species. Type, Arctobiella obscura Coquillett.

Arctophila Schiner, Wien. Ent. Monatschr., vol. 4, p. 215, 1860. 2 species. Type, Syrphus bombiformis FALLen, by designation of Williston, Synops. N. Amer. Syrph., 1886, p. 158.

Ardoptera Macquart, Ins. Dipt. Nord France, separata, p. 105, 1827. 1 species. Type, Tachydromia irrorata FALLEN. Equals Dolichocephala MACQUART, 1823.

$\checkmark$ Arayra Macquart, Hist. Nat., Dipt., vol. 1, p. 456, 1834. 7 species. Type, Musca diaphana Fabricius, the first species, by designation of Rondani, Dipt. Ital. Prodr., yol. 1, 1856, p. 141. Equals Porphyrops Meigex, 1824.

Argyrella Desvordy, Hist. Nat. Dipt., vol. 2, p. 87, 1863. 1 species (as 2). Type, Musca leucocephala Rossi. Equals Metopia Meigen, 1803. 
Argyria Desvoidy, Hist. Nat. Dipt., vol. 2, p. 82, 1863. 2 spreies (as 6). Type, Musca leucocephula Ross1, the first species, by original designation. Equals Metopia MEIGEN, 1803.

$\checkmark$ Argyromabe Schlver, Wien. Ent. Monatschr., vol. 4, p. 51, 1860. 3 species. Type, Anthrox tripunctata Wiedemans, the first species, by present designation. Equals Anthrax SCOPOL1, 1763.

J Argyrophylax Brauer and Bergenstamm, Denkschr. Kais. Akad. Wiss. Wien, vol. 56, p. 163, 1889. 1 species. Type, Tachina allincisa Wiedemann. Equals Sturmia Desvoiny, 1830.

- Argyrospyla Rondan, Dipt. Ital. Prodr., vol. 1, p. 162, 1856. 1 species. Type, Anthrax jucchus Fabricius. Equals Mima Meigen, 1820.

$\checkmark$ Aricia Desvondr, Essai Myorl., p. 486, 1830. 9 supposed species. Type, Musca impuncte FALLEN, the first 5 supposed species; by designation of Coquilletr, Journ. N. Y. Ent. Soc, vol. 9, 1901, p. 135. Equals Mydæa Desvoidy, 1830.

$\checkmark$ Arina Desvoldr, Essai Myod., p. 696, 1830. 1 species. Type, Arina olscura Desvoldy: Equals Chetocera Desvoldy, 1830.

- Arisbara Desroldr, Hist. Nat. Dipt., vol. 2, p. 290, 1863. 1 species. Type, Arisbxa lateralis Desvoidy. Equals Eliozeta Rondani, 1556.

- Arnoldia Kieffer, Wien. Ent. Zeit., rol. 14, p. 7, 1895. 2 species. Type, Cecidomyiu quercus Bixver, the second species, by present designation.

- Arpagita Liox, Atti Inst. Veneto, ser. 3, vol. 9, p. 1327, 1864. 1 species. Type, Stomoxys dorsulis Fabricius. Equals Dalmannia Desvoidir, 1830.

-Amenopms Brauer and Bergenstam, Denkschr. Kais. Akad. W'iss. Wien, vol, 58, p. 360, 1s91. 2 speries. Type, Sphixapata piligena Rondani, the second species, by designation of Bracer and Bergenstamu, Verh. Zool.-Bot. Ges. Wien, vol. 43, 1\$93, p. 504. Equals Senotainia M.ıсеиart, 1846.

- Arrhenica Osten Sacken, Proc. Acad. Nat. Sci. Phila. for 1859, p. 243, 1860. 2 species. Type, Arrhenica spinosa Osten SAcken, the first species, by present designation. Equals Caloptera Gueris, 1829.

$\checkmark$ Arribalzagia Tneobann, Monogr. Culic., vol. 3, p. 81, 1903. 1 species. Type, Arribalzugia maculipes Theoвald. Equals Cellía Theoвald, 1902.

- Artemita Walker, List Dipt. Brit. Mus., vol. 5, Suppl. 1, p. 61, 1854. 2 species. Type, Clitellaria amemides WaLker, the serond species, by designation of Braver, Denkschr. Kais. Akad. Wiss. Wien, vol. $t 4,1882$, p. 86. Syn., Acanthina Wienemans, 1830, preoccupied; Acanthinomyia Hunter, 1900.

- Arthria Kinkry, Fauna Bor. Amer., Ins., p. 311, 1837. 1 species. Type, Arthria analis Kirby. Equals Aspistes Meigen, 1818.

$\checkmark$ Arthroceras Williston, Ent. Amer., vol. 2, p. 107, 1886. 2 species. Type, Arthroceras pollinosus WiLliston, the second species, by present designation.

- Arthrochæta Bracer and Bergenstam, Denkschr. Kais. Akad. W'iss. Wien, vol. 56, p. 134, 1889. 1 species. Type, Arthrochatr demoticoides Brauer and Bergenstam. Syn., Microtrichomina Giglio-Tos, 1893.

- Arthrocnodax RübsaAmen, Wien. Ent. Zeit., vol. 14, p. 189, 1895. 3 species. Type, Aithrocnodax vitis RüBsAAMEn, the first species, by present designation.

- Arthropeas Loew, Ent. Zeit. Stettin, vol. 11, p. 304, 1850. 1 species. Type, Arthropeas sibirica LoEw.

- Arthrostylum Williston, Kansas Univ. Quart., vol. 4, p. 108, 1895.1 species. Type, Pheneus tibialis Walker (as fascipennis, new species). Equals Pheneus Walker, 1851.

- Ascia Meigen, Syst. Beschr. Zweifl. Ins., vol. 3, p. 185, 1822. 5 species (as 9). Type, Syrphus podagricus FABricus, the first species, by designation of IVESTWood, Intr., vol. 2, 1840, Synops., p. 136. Not Ascia Scopoli, 1777. Equals Neoascia Williston, 1886. 
$\checkmark$ Asemosyrphus Bigot, Bull. Soc. Ent. France for 1882, p. 128, 1882. 2 species (as 4). Type, Helophilus mexicanus Macquart (equals the first 3 species), by present designation.

- Asilus Linn.eus, Syst. Nat., 10th ed., p. 605, 1758. 12 species. Type, Asilus crabroniformis Linn.eus, the third species, by designation of LATREILle, Consid. General., 1810, p. 443.

- Asindulum Latreille, Hist. Nat. Crust. et Ins., vol. 14, p. 290, 1805. 1 species. Type, Asindulum nigrum Latkeille. Syn., Macrorrhyncha Winnertz, 1846; Adelinia Costa, 1857; Antlemon Loew, 1871.

$\checkmark$ Asphondylia Loew, Dipt. Beitr., vol. 4, pp. 20, 21, 1850. 1 species. Type, Cecidomyia sarothamni Loew. Syn., Phyllophaga Rondani, 1856.

$\checkmark$ Aspidoptera Coquillett, Can. Ent., vol. 31, p. 334, 1899. 1 species. Type, Aspidoptera busckii Coquillett. Syn., Lepopteryx Speiser, 1900.

$\checkmark$ Aspilia Rovdani, Atti Soc. Ital. Sci. Nat., vol. 9, p. 3, 1866. 6 species. Type, Anthomyia allotalla Meigen, the sixth species, by original designation. Equals Mydæa Desvoidy, 1830.

Aspilota Loww, Monogr. Dipt. N. Amer., vol. 3, p. 286, 1873. . 2species. Type, Trypeta alba LoEw, the first species, by present designation. Not Aspilota Foerster, 1862. Equals Neaspilota Osten SACKen, 1878.

$\checkmark$ Aspistes Mergen, Syst. Beschr. Zweifl. Ins., vol. 1, p. 319, 1818. 1 species. Type Aspistes berolinensis Meigen. Syn., Arthria Kinby, 1837.

$\checkmark$ Asteia Meigen, Syst. Beschr. Zweifl. Ins., vol. 6, p. 88, 1830. 2 species. Type, Asteia amona MeIGeN, the first species, by designation of WEstwood, Intr., vol. 2, Synops., 1840, p. 152.

$\checkmark$ Asthenia Westwood, Mag. Zool., ser. 2, vol. 4, Ins., pl. 94, 1842. 1 species. Type, Asthenia fasciala Westwood. Not Asthenia Hëвner, 1816. Equals Blepharicera Macquart, 1843.

$\checkmark$ Astoma Lioy, Atti Inst. Veneto, ser. 3, vol. 9, p. 762, 1864. 1 species. Type, Nemotelus niger De Gerr. Equals Omphraie Me1gen, 1800.

$\checkmark$ Astrophanes Osten Sacken, Biol. Centr.-Amer., Dipt., vol. 1, p. 106, 1886. 1 species. Type, Astrophanes adonis Osten Sacken.

$\checkmark$ Asynapta Loew, Dipt. Beitr., vol. 4, pp. 20, 21, 1850. 5 species. Type, Cecidomyia longicollis Loew, the first species, by present designation.

- Asyndetus Loew, Berliner Ent. Zeitschr., vol. 13, p. 35, 1869. 2 species. Type, Asyndetus ammophilus LoEw, the first species, by present designation.

$\checkmark$ Atacta Schiner, Reise Novara, Zool., vol. 2, Dipt., p. 328, 1868. 1 species. Type, Atacta brasiliensis Schiner.

$\checkmark$ Atalanta Meigen, Nouv. Class. Mouch., p. 31, 1800.* No species. Clinocera Meigen, 1803, is a change of name. Type, Clinocera nigra Meigen. Syn., Clinocera Meigen, 1803; Paramesia Macquart, 1835.

$\checkmark$ Atarba Osten SAckein, Monogir. Dipt. N. Amer., vol. 4, p. 127, 1869. 1 species. Type, Atarba picticornis Osten SAcken.

$\checkmark$ Atelenevra Macquart, Rec. Soc. Sci. Lille for 1834, p. 356, 1834. 1 species. Type, Pipunculus holosericeus Meigen. Equals Chalarus Walker, 1834.

$\checkmark$ Atelestus Walker, Ent. Mag., vol.4, p. 229, 1837. 1 species. Type, Empis pulicaria Falden (as sylvicola, new species). Syn., Platycnema Zetterstedt, 1838.

$\checkmark$ Ateloglossa Coquillett, Journ. N. Y. Ent. Soc., vol. 7, p. 219, 1899. 1 species. Type, Ateloglossa cinerea Coquillett.

Atemnocera Bigot, Bull. Soc. Ent. France for 1882, p. 114, 1882. 1 species. Type, Volucella scutellata Macquart. Equals Volucella Geoffror, 1762.

Ateria Desvoidy, Hist. Nat. Dipt., vol, 1, p. 809, 1863. 1 species. Type, Ateria nitida Desvordy. Equals Wagneria Desvordy, 1830.

$\mathcal{A}$ Atherix MEIGEN, Illiger's Mag., vol. 2, p. 271, 1803. 2 species. Type, Sylvicolæ melanchoila HaRRIS (as Rhagio diadema FABRICIUS), the first species, by designation of 
Latreille, Consider Général., 1810, p. 443. Syn., Nodutis Meigex, 1820; Ibisia RONDANI, 1856.

$\checkmark$ Athrycia Desvordy, Essai Myod., p. 111, 1830. 2 species. Type, Athrycia erythrocerc Desvordy, the first species, hy designation of Desvordy, Hist. Nat. Dipt., vol. 1, 1863, p. 830 . Equals Voria Desrordy, 1830.

$\checkmark$ Athyroglossa Loew, Neue Beitr., vol. 7, p. 12, 1860. 1 species. Type, Notiphila glabra MEIGEN.

- Atilia Destony, Hist. Nat. Dipt., vol. 1, p. 475, 1863. 1 species (as 5). Type, Musca libatrix Paxzer. Equals Zenilla Desvoidy, 1830.

- Atomuria B1Got, Ann. Soc. Ent. France for 1854, p. 482, 185t. 1 species. Type, Tipula atomariu De Geer. Not.Itomaria Stephens, 1830. Equals Epidapus Haliday, 1856.

$\checkmark$ Atomoguster Micquart, Hist. Nat., Dipt., vol. 2, p. 329, 1835. 6 species. Type, Atomogaster macquarti STEGER (as Anthomyia triquetra ME1GEN), the first species, by original designation. Equals Azelia Desroidr, 1830.

v Atomosia Macquart, Dipt. Exot., vol. 1, pt. 2, p. 73, 1838. 6 species. Type, Laphria puella WredeMans (as incisuralis, new species), the fourth species, by present designation.

$\checkmark$ Atonia Wrlliston, Psyche, vol. 5, p. 257, 1889. 2 species. Type, Atomosia mikii Willistox, the second species, by designation of Willistox, Biol. Centr.-Amer., Dipt., vol. 1, 1901, p. 316.

- Atractia Macquart, Dipt. Exot., vol. 1, pt. 2, p. 151, 1838. 1 species. Type, Asilus psilogaster WiedemaxN.

$\checkmark$ Atractocera Mrigex, Illiger's Mag., vol. 2, p. 263, 1803. 1 species. Type, Simulium ornatum Meigen (as Tipula regelationis Linneus). Equals Molusina MeIgex, 1800.

$\checkmark$ Atrichia Scllraxk, Fauna Boica, vol. 3, p. 54, 1803.* 1 species (as 3). Type, Musca fenestralis Linners. Equals Omphrale Meigex, 1800.

- Atrichia Loew, Berliner Ent. Zeitschr., vol. 10, p. 42, 1866. 1 species. Type, Atrichia longurio Loew. Not Atrichia Schrank, 1803. Equals Pseudatrichia Osten SACKEN, 1877.

- Atrichopogon Kieffer, Gen. Ins., Dipt., Chir., p. 53, 1906. 3 species. Type, Ceratopogon exilis Coquilletr, the first species, by present designation. Equals Helea Meigex, 1800.

- Atropharista Townsend, Trans. Amer. Ent. Soc., vol. 19, p. 92, 1892. 1 species. Type, Tachina insolita Walker (as jurinoides, new species). Equals Melanophrys Williston, 1886.

- Atrophopalpus Towssend, Ent. News, vol. 3, p. 130, 1892. 1 species. Type, Atrophopulpus angustigornis Towxsend.

- Atrophopoda Townsexd, Trans. Amer. Ent. Soc., vol. 18, p. 373, 1891. 1 species. Type, Atrophopode singularis Towrsexd. Equals Paradidyma Brauer and BergeNSTAMM, 1891.

v. Atylotus Ostex SAcken, Mem. Boston Soc. Nat. Hist., vol. 2, p. 426, 1876. 4 species. Type, Tabanus bicolor Wiedemann, the first species, by present designation. Equals Tabanus Lins ets, 1758.

$\checkmark$ Aulacigaster Macquart, Hist. Nat., Dipt, vol. 2, p. 579, 1835. 1 species. Type, Aulacigaster mefitarsis Macruart. Syn, Apotomella Dufour, 1846; Ampycophora WAALBERG, 1847.

J Automola Loew, Monogr. Dipt. N. Amer., vol. 3, p. 118, 1873. 2 species. Type, Ortalis atomaria WIEDEMAN, the first species, by present designation.

$\checkmark$ Avihospita Hexdel, Wien. Ent. Zeit., vol. 20, p. 29, 1901. 1 species. Type, Lusca azurea Fallex. Equals Protocalliphora Hough, 1899.

$\checkmark$ Azelia Desiromy, Essai Myod., p. 592, 1830. 9 supposed species. Type, Anthomyia triquetra WIEDEMANx (equals the first and fourth supposed species), by designation of Rondani, Atti Soc. Ital. Sci. Nat., vol. 9, 1866, p. 72. Syn., Atomogaster Macquart, 1835. 
Baccha Farricius, Syst. Antliatorum, p. 199, 1805. 6 species. Type, Syrphus elongatus FABRicius, the last species, by designation of Curtis, Brit. Ent., p. 737, 1839. Bacchis Desvoidy, Essai Myod., p. 803, 1830. 5 species. Type, Bacchis cellarum Desvoidr, the first species, by present designation. Equals Leptocera OLIvier, 1813. Bactria Mleigen, Syst. Beschr. Zweifl. Ins., vol. 2, p. 307, 1820. 1 species. Type, Asilus pictus Meigen. Syn., Trupanea Macquart, 1838; Promachus Loew, 1848; Telejoneura Rondani, 1864.

Baldratia Kreffer, Meine Antwort Rübsaamen, p. 6, 1897.* 1 species. Type, Baldratia salicornix KIEFFER.

Balioptera Loww, Berliner Ent. Zeitschr., vol. 8, p. 347, 1865. 6 species. Type, Musca combinata Linnæus, the first species, by present designation. Equals Geomyza FALLEN, 1810.

Barpleygma Wulp, Biol. Centr.-Amer., Dipt., vol. 2, p. 416, 1899. 1 species. Type, Baryplegma gilva WuLP.

$\checkmark$ Bathydexia Wulp, Biol. Centr.-Amer., Dipt., vol. 2, p. 222, 1891. 2 species. Type, Phorostoma appendiculata Вigot, the first species, by present designation.

$\checkmark$ Baumhaueria Meigen, Syst. Beschr. Zweifl. Ins., vol. 7, p. 251, 1838. 1 species. Type, Tachina gonixformis Meigen. Syn., Pachycephala Liov, 1864.

Bebricia Desvoiny, Hist. Nat. Dipt., vol. 1, p. 1112, 1863. 2 species. Type, Macquartia microcera DesvoIdy, the first species, by original designation. Equals, Macquartia DesvoIDy, 1830.

Beggiatia Lroy, Atti Inst. Veneto, ser. 3, vol. 9, p. 1088, 1864. 1 species. Type, Sepsis barbipes Meigen. Equals Sepsis Fallen, 1810.

Belida Desvoidy, Hist. Nat. Dipt., vol. 2, p. 45, 1863. 1 species. Type, Belida flavipalpis Desvordy. Equals Sturmia Desvoldy, 1830.

Bellardia Desvoidy, Hist. Nat. Dipt., vol. 2, p. 548, 1863.1 species. Type, Tachina obsoleta Meigen (as vernalis, new species). Equals Onesia Desvoidy, 1830.

- Bellieria Desvordy, Hist. Nat. Dipt., vol. 2, p. 432, 1863. 1 species (as 2). Type, Sarcophaga melanura Meigen. Equals Sarcophaga Meigen, 1826.

Belvosia Desvordr, Essai Myod., p. 103, 1830. 1 species. Type, Musca bifasciata Fabricius (as bicincta, new species). Syn., Latreillia Desvoidy, 1830; Latreillimyia Townsend, 1908.

$\checkmark$ Bercoa Desvoldy, Hist. Nat. Dipt., vol. 2, p. 549, 1863. 8 species (as 13). Type, Musca hamorrhoidulis Faldev, the sixth species, by original designation. Equals Sarcophaga Meigen, 1826.

Beris_Latreille, Hist. Nat. Crust. et Ins., vol. 3, p. 447, 1802. 1 species. Type, Musca chalybeata Forster (as Stratiomys sexdentata Fabricius). Syn., Hexacantha Meigen, 1803; Oplachantha Rondani, 1864; Hexacantha Lioy, 1864; Octacantha Lioy, 1864 .

Berismyia Giglio-Tos, Boll. Mus. Zool. Univ. Torino, vol. 6, No. 108, p. 2, 1891. No species. In Mem. Reale Accad. Sci. Torino, ser. 2, vol. 43, 1892, 1 species. Type, Berismyia fusca Giglo-Tos.

Bertieria KIEFFer, Bull. Soc. Ent. France for 1896, p. 189, 1896. 1 species. Type, Bertieria gemmicola Kieffer. Equals Rabdophaga Westwood, 1847.

Beskia Brauer and Bergenstamm, Denkschr. Kais. Akad. Wiss. Wien, vol. 56, p. 139, 1889. 1 species. Type, Tachina ælops WALKER (as cornuta, new species). Syn., Ocypterosipha Townsend, 1894.

Besseria Desvoidy, Essai Myod., p. 232, 1830. 1 species. Type, Ocyptera lateralis Fallen (as reflexa, new species). Syn., Wahlbergia Zetтerstedt, 1842; Apostrophus Loew, 1870.

Bezzia KiefFer, Bull. Soc. Ent. France for 1899, p. 69, 1899. 1 species. Type, Ceratopogon ornatus Meigen. Syn., Probezzia Kiefrer, 1906.

$$
\text { Proc.N.M.vol.37-10-33 }
$$


Bibio Geofrroy, Hist. Abrégée Ins., vol. 2, p. 568, 1762. 5 species. Type, Tipula hortulana Linnsus, the third species, by designation of Latreille, Consider. Général., p. 442, 1810 . Syn., Pullata Harris, 1776.

$\checkmark$ Bibiocephala Osten Sacken, Ann. Rep. U. S. Geol. Surv. for 1873, p. 564, 1874. 1 species. Type, Bibiocephala grandis Osten Sacken. Syn., Agathon Röder, 1890.

Bibioides Coquillett, Proc. Ent. Soc. Washington, vol. 6, p. 171, 1904. 1 species. Type, Bibioides halteralis CoquilletT.

- Bicellaria Macquart, Monogr. Empides, p. 155, 1823. 1 species. Type, Empis spuria Fallex (as nigra, new species). Syn., Cyrtoma Meigen, 1824.

- Bigotia Desvordy, Hist. Nat. Dipt., vol. 1, p. 1048, 1863.1 species. Type, Tachina simulans Meigen (as brevicornis MACQUart). Equals Exorista Meigen, 1803.

- Biomya Rondani, Dipt. Ital. Prodr., vol. 1, p. 72, 1856. No species. Tiviania Rondani, 1861, was a change of name. Type, Tachina cinerea Fallen. Syn., Fabricia Meigen, 1838 (preoccupied); Viviania Rondani, 1861; Masiphya Brauer and Bergenstami, 1891.

- Bischofia Hendel, Abh. Zool.-Bot. Ges. Wien, vol. 2, p. 52, 1902.3 species. Type, Sciomyza simplex FaLLen, the first species, by present desig ation. Equals Sciomýza Fallen, 1820.

- Bittacomorpha Westwood, Lond. Edinb. Philos. Mag., vol. 6, p. 281, 1835.1 species. Type, Tipula clavipes Fabricius.

- Blacodes Loew, Berliner Ent. Zeitschr., vol. 18, p. 377, 1874. Change of name for Blax Loew, 1872, not Thomson, 1860. Type, Blax bellus Loew. Not Blacodes Dejean, 1859, Equals Cophura Osten Sacken, 1887.

$\checkmark$ Blax Loew, Berliner Ent. Zeitschr., vol. 16, p. 241, 1872. 1 species. Type, Blax bellus Loew. Not Blax Thomson, 1860. Equals Cophura Osten Sacken, 1887.

$\checkmark$ Blepharepium Rondani, Studi Ent., vol. 1, p. 89, 1848. 1 species. Type, Blepharepium luridum Rondani. Syn., Planetolestes Arribalzaga, 1879.

$\checkmark$ Blepharicera Macquart, Ann. Soc. Ent. France for 1843, p. 61, 1843. 1 species. Type, Asthenia fasciata Westwood (as limbipennis, new species). Syn., Asthenia WEstwood, 1842, preoccupied.

- Blepharidea Rondani, Dipt. Ital. Prodr., vol. 1, p. 67, 1856. 1 species. Type, Tachina vulgaris Fallen. Equals Phryxe Desvordy, 1830.

- Blepharidopsis Brauer and Bergenstamm, Denkschr. Kais. Akad. Wiss. Wien, vol. 58, p. 329, 1891. 1 species. Type, Tachina nemea Meigen. Equals Phryxe DesvoIDY, 1830 .

- Blepharigena Rondani, Dipt. Ital. Prodr., vol. 1, p. 69, 1856. 1 species. Type, Tachina trepida Meigen. Equals Voria Desvoidy, 1830.

$\checkmark$ Blepharipa Rondani, Dipt. Ital. Prodr., vol. 1, p. 71, 1856.1 species. Type, Nemoræa seutellata Desvoidy (as Masicera ciliata M MCQUART). Equais Sturmia DesvoIDy, 1830.

$\checkmark$ Blepharipeza Macquart, Dipt. Exot., vol. 2, pt. 3, p. 211, 1843. 1 species. Type, Tachina leucophrys Wiedemann (as rufipalpis, new species). Syn., Rileya Brauer and Bergenstami, 1893; Rileymyia Townsend, 1893.

- Blephariptera Macquart, Hist. Nat., Dipt., vol. 2, p. 412, 1835.12 species. Type, Musca serrata Linneus, the second species, by designation of Westwood, Intr., yol. 2, Synops., 1840, p. 145 . Equals Heleomyza FALLen, 1810.

Blepharoneura LoEw, Monogr. Dipt. N. Amer., vol. 3, p. 272, 1873. 1 species. Type, Trypeta precilogaster Loew.

Blepharoprocta Loew, Berliner Ent. Zeitschr., vol. 6, p. 194, 1862. 3 species. Type, Brachystoma nigrimana LoEw, the first species, by designation of CoquilletT, Proc. Ent. Soc. Washington, vol. 5, p. 246, 1903.

$J$ Blissonia Desvordy, Hist. Nat. Dipt., vol. 2, p. 648, 1863. 1 species (as 3). Type, Muscu assimilis Fallen. Equals Muscina Desvoidy, 1830. 
Blondelia Desvordy, Essai Myod., p. 122, 1830. 2 species (as 4). Type, Tachina nigripes FALLEN, the first two and the last supposed species, by present designation. Equals Lydella Desvoidr, 1830.

Blumia Desvordy, Hist. Nat. Dipt., vol. 1, p. 468, 1863. 1 species. Type, Tachina vulgaris Fallen (as occlusa, new species). Equals Phryxe Desvoidr, 1830.

Bogeria Austen, Ann. Mag. Nat. Hist. for 1895, p. 391, 1895. 1 species. Type, Bogeria princeps Austen.

Bolbomyia Loew, Bernst. und Bernstein-fauna, p. 39, 1850** No species. In 1862, 1 species. Type, Bolbomyia nana Loww.

- Boletina St MEIGen, the first species, by designation of Johannsen in Wytsman's Gen. Ins., Dipt., Mycet., 1909, p. 73.

- Bolitophila Meigen, Syst. Beschr. Zweifl. Ins., vol. 1, p. 220, 1818. 1 species. Type, Macrocera hybrida Meigen (as fusca, new species). Syn., Messala Curtis, 1836.

$\checkmark$ Bolomyia Brauer and Bergenstami, Denkschr. Kais. Akad. Wiss. Wien, vol. 58,

p. 347,1891 . 1 species. Type, Exorista rufata Bigot (as Mystacella violacea WulP).

Bombibia Lioy, Atti Inst. Veneto, ser. 3, vol. 9, p. 1326, 1864. 1 species. Type,

Conops flavipes Linneus. Equals Conops Linnzeus, 1758.

- Bornbyliomyia Brauer and Bergenstamm, Denkschr. Kais. Akad. Wiss. Wien, vol. 56, p. 131, 1889. 1 species. Type, Hystricia flavipalpus Macquart.

Bombylius Linneus, Syst. Nat., 10th ed., p. 606, 1758. 3 species. Type, Bombylius major Linneus, the first species, by designation of Latreille, Consider. Général., 1810, p. 443.

$\checkmark$ Bonellia Desvordy, Essai Myod., p. 56, 1830. 1 species (as 3). Type, Tuchina hæmorrhoidalis Fallen. Equals Linnæmya Desvoidy, 1830.

Bonnetia Desvordy, Escai Myod., p. 55, 1830. 1 species (as 2). Type, Tachina comta Fallen. Syn., Marshamia Desvoidr, 1830; Micropalpis Macquart, 1834.

Bophrosia Rondani, Dipt. Ital. Prodr., vol. 1, p. 183, 1856. 1 species. Type, Limonia immaculata Meigen. Equals Tricyphona Zetterstedt, 1837.

Borlensued I eig Ex, Illiger's Mag., vol. 2, p. 276, 1803. No species. In 1830, 29 species. Type, Musca subsultans FABricius, the second species, by designation of CurTis, Brit. Ent., 1833, p. 469. Eqwals Cypsela Meigen, 1800. (B. Borborus of authors equals Copromyza FALLEN.

$\checkmark$ Boreodromia Coquillett, Proc. Ent. Soc. Washington, vol. 5, p. 247, 1903.1 species. Type, Synamphotera bicolor Loew.

Botanobia Lioy, Atti Inst. Veneto, ser. 3, vol. 9, p. 1125, 1864. 1 species (as 2). Type, Oscinis dubia Macquart. Syn., Oscinisoma Lior, 1864; Oscinimorpha Lioy, 1864; Macrostyla Lioy, 1864.

- Botanophila Lroy, Atti Inst. Veneto, ser. 3, vol. 9, p. 990, 1864. 1 species. Type, Anthomyia varicolor Meigen. Equals Pegomya Desvoidy, 1830.

Brachicoma Rondani, Dipt. Ital. Prodr., vol. 1, p. 69, 1856.1 species. Type, Tachina devia Fallen (as nitidula Meigen). Syn., Oppia Desvoidy, 1863; Laccoprosopa Townsend, 1891; Sarcotachinella Townsend, 1892.

7 Brachiomyia TнеовацD, Monogr. Culic.; vol. 2, p. 343, Nov. 23, 1901. 1 species. Type, Deinocerites cancer Theoвald (as magna, new species). Equals Deinocerites TheовaLn, July 15, 1901.

Brachiophyra Giglio-Tos, Boll. Mus. Zool. Torino, vol. 8, No. 147, p. 9, 1893. 1 species. Type, Brachiophyra effrons Giglio-Tos.

$\checkmark$ Brachiosoma Theobald, Journ. Trop. Med. for 1901, p. 235, July 15, 1901. No species. Brachiomyia Th вовацд, November 23,1901 , is evidently a change of name. Type, Deinocerites cancer Theobald. Equals Deinocerites Theobald, July 15, 1901.

Brachycampta Winnertz, Verh. Zool.-Bot. Ges. Wien, vol. 13, p. 833, 1863. 8 species. Type, Mycetophila alternans ZETTERstedT, the first species, by present des: ignation. Equals Allodia Winnertz, 1863. 
Brachydeutera LoEw, Monogr. Dipt. N. Amer., vol. 1, p. 162, 1862. 1 species. Tỷpe, Notiphila argentata W ALK ER (as dimidiata, new species).

$\checkmark$ Brachygaster MElGEN, Syst. Beschr. Zweifl. Ins., vol. 5, 1. 244, 1826.1 species (as 2). Type, Cordilura analis Meigen. Not Brachygaster Leac11, 1817. Equals Mycetaulus Loew, 1845.

$\checkmark$ Brachymyia Williston, Can. Ent., vol. 14, p. 77, 1882. 2 species. Type, Brachymyia lupina Williston, the first species, by present designation. Equals Penthesilia MEIGEN, 1800.

v Brachyneura Rondani, Mem. 2a. Serv. Ditt. Ital., p. 18, 1840.* 1 species. Type, Brachyneura fuscogrisea RoNDAN1.

$\checkmark$ Brachyopa Melgen, Syst. Beschr. Zweifl. Ins., vol. 3, p. 260, 1822. 3 species. Type, IIusca conica PANzer, the first species, by designation of Westwoon, Intr., vol. 2, Synops., 1840, p. 137. Syn., IIammerschmidtia Schummel, 1834; Exocheila Rondani, 1857; Eugeniamyia Williston, 1882.

- Brachypalpus Macquart, Hist. Nat., Dipt., vol. 1, p. 523, 1834. 3 species (as 5). Type, Syrphus valgus PANzer, the last species (as tuberculutus), by designation of RoNDANI, Nuovi Ann. Soc. Nat., 1844, p. 456.

- Brachypremna Osten SACKen, Berliner Ent. Zeitschr., vol. 30, p. 161, 1886. 2 species. Type, Tipula dispellans WALKER, the first species, by present designation.

$\checkmark$ Brachypteromyia IVilliston, Ent. News, vol. 7, p. 184, 1896. 1 species. Type, Anapera fimbriala IV ATERHouse (as femorata, new species).

$\checkmark$ Brachypus Meigen, Syst. Beschr. Zweifl. Ins., vol.4, p. 34, 1824. 1 species. Type, Diaphorus cyanocephalus Meigen. Equals Diaphorus Meigen, 1824.

- Brachystoma Meigen, Syst. Beschr. Zweifl. Ins., vol. 3, p. 12, 1822. 2 species. Type, Syrphus vesiculosus Fabricius, the second species, by designation of Blanch ARD, Hist. Nat. Ins., vol. 3,1840 , p. 582.

- Brachystomus Costa, Il ( iiamb. Vico, Napoli, vol. 2, p. 445, 1857.* 1 species. Type, Tabanus gigas Herbst (as ursus, new species). Equals Tabanus Linn eus, 1758.

- Brachystylum Macquart, Ann. Soc. Ent. France for 1855, p. 199, 1855. 1 species. Type Brachystylum nigra Macquart. Equals Wagneria Desvoidy, 1830.

- Brauerimyia Townsend, Smiths. Misc. Coll., vol. 51, p. 65, 1908. Change of name for Wulpia Brauer and Bergenstamm, 1893, not of Bigot, 1886. Type, Iulpia aperta Brauer and Bergenstam. Equals Vanderwulpia Townsend, 1891.

$\checkmark$ Bremia Rondan1, Atti Soc. Ital. Nat., vol. 2, p. 289, 1861. 1 species. Type, Diplosis decorata Loww.

$\checkmark$ Bricinnia I'ALkER, Trans. Ent. Soc. Lond., 11. ser., vol. 5, p. 324, 1861.1 species. Type, Bricinnia flexinitta WALKER.

- Bricinniella Ġiglo-Tos, Boll. Mus. Zool. Univ. Torino, vol. 8, No. 158, p. 13, 1893. 1 species. Type, Bricinniella cyunea GiıLIo-Tos.

$\checkmark$ Brontza Kowarz, Verh. Zool.-Bot. Gies. Wien, vol. 23, p. 461, 1873. 2 species. Type, Anthomyiu polystigma Meicien, the first species, by present designation. Equals Limnophora Desvoldy, 1830.

- Bryocrypta KıefFer, Berliner Ent. Zeitschr., vol. 41, p. 8, 1891. 1 species. Type, Bryocrypta dubia K1EFFER.

$\checkmark$ Bucentes Latreille, Gen. Crust. et Ins., vol. 4, p. 339, 1809. 1 species. Type, Musca geniculata De GeEr (as cinereus, new species). Equals Crocuta M IEIGEN, 1800.

- Byomya Desvoiny, Essai Myod., p. 392, 1830. 2 species (as 3). Type, IIusca tempestiva FalLEN, the first and last supposed species, by present designation. Equals Musca Linnaus, 1758.

- Cacomyia Coqulllett, Class. Mosq. Amer., p. 16, 1906. 2 species. Type, Homagogus albomaculatus ThеоваLD, the first species, by present designation.

$\checkmark$ Cacoxenus Loew, Wien. Ent. Monatschr., vol. 2, p. 217, 1858. 1 species. Type, Cacoxenus indagator LoEw. 
Cinnis Desvordy, Hist. Nat. Dipt., vol. 1, p. 675, 1863. 2 species. Type, Cænis prompta Desvordy, the first species, by original designation. Equals Ceranthia Desvoidy, 1830.

- Calirrhoe Meigen, Nouv. Class. Mouch.,p. 39, 1800** No species. Equals Prosena St. Fargeau and Serville, 1828. Type, Stomoxys siberita Fabricius. Syn., Prosena St. FARGeAU and Servizle, 1828.

- Callicera Panzer, Fauna Ins. Germ., heft 104, no. 17, 1806. 1 species. Type, Bibio rnea FABricius.

- Callinicus Loww, Berliner Ent. Zeitschr., vol. 16, p. 247, 1872. 1 species. Type, Callinicus calcaneus Loew.

- Calliphora.Desvordy, Essai Myod., p. 433, 1830. 17 species. Type, Musca erythrocephala Meigen, by original designation (as vomitoria Linneus). Syn., Compsomyia Rondani, 1875; Eucalliphora Townsend, 1908.

$\checkmark$ Callomyia Meigen, Klass. Beschr. Zweifl. Ins., p. 311, 1804. 1 species. Type, Callomyia elegans Meigen. Equals Cleona Meigen, 1800.

- Callomyza Fallen, Platyp. Bombyl. Sveciæ, p. 6, 1815. 2 species. Type, Heteroneura leptiformis FaLLEN, the first species, by designation of ZETTERSTEDT, Dipt. Scand., vol. 3; 1844, p. 910. Equals Cleona Meigen, 1800.

- Callopistria Loew, Monogr. Dipt. N. Amer., vol. 3, p. 140,1873. 1 species. Type, Platystoma annulipes Macquart. Not Callopistria HüBner, 1816. Equals Callopistromyia Hendel, 1907.

- Callopistromyia Hendel, Wien. Ent. Zeit., vol. 26, p. 98, 1907. Change of name for Callopistria Loew, 1873, not of HüBner, 1816. Type, Platystoma annulipes MACQUART. Syn., Callopistria Loew, 1873, preoccupied.

Calobata Meigen, Illiger's Mag., vol. 2, p. 276, 1803. 2 species. Type, Musca petronella LINN Lus, the second species, by designation of WESTwood, Intr., vol. 2, Synops., 1840, p. 148. Equals Trepidaria Meigen, 1800.

Calodexia Wulp, Biol. Centr.-Amer., Dipt., vol. 2, p. 257, 1891. 3 species. Type, Calodexia majuscula WulP, the first species, by present designation.

Caloptera Guerin, Voy. Monde Coquille, Zool., Plates, Ins., No. 20, fig. 2, 1829. 1 species.' Type, Caloptera fasciata Guerin. Syn., Evanioptera Guerin, 1838; Eriocera Macquart, 1838; Pterocosmos Walker, 1848; Oligomera Doleschall, 1857; Physecrania Bigot, 1859; Arrhenica Osten SACken, 1860.

$\checkmark$ Calopterella, new genus. Type, Diastata vagans Loew. Syn., Trichoptera Liov, 1864, preoccupied.

$\checkmark$ Calotarsa Townsend, Can. Ent., vol. 26, p. 50, 1894. 1 species. Type, Platypeza pallipes Loew (as ornatipes, new species). Equals Clythia MeIGen, 1800.

$\checkmark$ Calypiia Desvordy, Hist. Nat. Dipt., vol. 2, p. 576, 1863. 1 species (as 2). Type, Sarcophaga melanura Meigen. Equals Sarcophaga Meigen, 1826.

$\checkmark$ Calyptidia Desivordy, Hist. Nat. Dipt., vol. 2, p. 59, 1863. 1 species. Type, Calyptidia occlusa Desvordy: Equals Leucostoma Meigen, 1803.

$\checkmark$ Camarona Wulp, Biol. Centr.-Amer., Dipt., vol. 2, page 241, 1891. 2 species. Type, Camarona xanthogastra WuLP, the first species, by present designation.

$\gamma$ Camerania Giglio-Tos, Boll. Mus. Zool. Univ. Torino, vol. 6, No. 117, p. 3, 1892. 1 species. Type, Camerania macrocephala Giglio-Tos (as Temnocera megacephala Loew?). Equals Volucella Geoffroy, 1762.

$\checkmark$ Camill.a Desvordy, Hist. Nat. Dipt., vol. 2, p. 641,1863. 3 species. Type, Morellia aenescens Desvordy, the first species, by present designation. Equals Morellia Desvoldy, 1830.

r Campeprosopa Macquart, Dipt. Exot., Suppl. 4, p. 46, 1850. 1 species. Type Campeprosopa flavipes MACQUART.

Campiglossa Rondani, Bull. Soc. Ent. Ital., vol. 2, p, 121, 1870. 1 species. Type, Tephritis irrorata FAllen. Equals Spathulina Rondani, 1856. 
Campineura Rondani, Dipt. Ital. Prodr., vol. 1, p. 52, 1856. 1 species. Type, Chrysogaster frontalis LoEw (as venusta, new species). Equals Chrysogaster MEIGEN, 1800.

- Campsicnemus Halidar, in Walker's Ins. Britt., Dipt., vol. 1, p. 187, 1851. 6 species. Type, Dolichopus curvipes Fallen, the second species, by present designation. Equals Camptosceles HaLiday, 1831.

$\checkmark$ Camptocladius Wulp, Tijdschr. Ent, vol. 17, p. 133, 1874. 3 species. Type, Tipula byssina Schrank, the first species, by present designation.

$\checkmark$ Camptoneuromyia Felt, 23d Rep. State Ent. N. Y., p. 334, 1908. 5 species. Type, Dasineura virginica FELT, the first species, by present designation.

$\checkmark$ Camptonerra Macquart, Dipt. Exot., vol. 2, pt. 3, p. 200, 1843. 2 species. Type, Musca picta FABricius, the first species, by original designation. Equals Delphinia DEsvoIDY, 1830.

$\checkmark$ Camptoprosopella Hendel, Wien. Ent. Zeit., vol. 26, p. 223, 1907. 3 species. Type, Camptoprosopella melanoptera HeNDEL, the first species, by present designation.

$\checkmark$ Camptosceles Haliday, Zool. Journ., vol. 5, p. 357, 1831. 4 species. Type, Dolichopus scambus Fallen, the first species, by present designation. Syn., Campsicnemus HALIDAY, 1851.

Campylocheta Rondani, Dipt. Ital. Prodr., vol. 3, p. 157, 1859. 1 species. Type, Tachina obscura Falden (as Phorocera schistacen Meigen). Syn., Fallenia Meigen, 1838, preoccupied; Frivaldskia Schiner, 1861; Hypochæta Brater and Bergenstamm, 1889.

Campylomyza Meigen, Syst. Beschr. Zweifl. Ins., vol. 1, p. 101, 1818. 4 species. Type, Campylomyza flavipes MeIgev, by designation of WEsTwood, Intr., vol. 2, Synops., 1840 , p. 126.

$\checkmark$ Canace Haliday, Ann. Nat. Hist., vol. 3, p. 411, 1839. 1 species. Type, Ephydra nasica HALIDAY.

J Carbonia Desvoidy, Hist. Nat. Dipt., vol. 1, p. 806, 1863. 1 species. Type, Ocyptera costata FALlen (as impatiens, new species). Equals Wagneria Desvoidy, 1830.

$\int$ Carcelia Desvordy, Essai Myod., p. 176, 1830. 21 species. Type, Tachina gnava Meigen (as bombylans, new species), the second species, by designation of Desvoidy, Hist. Nat. Dipt., vol. 1, 1863, p. 220. Syn., Chetoliga Rondani, 1856; Parciëxorista Brauer and Bergenstama, 1889; Sisyropa Brauer and Bergenstamm, 1889; Eusisyropa Townsend, 1908.

f Cardiacephala Macquart, Dipt. Exot, vol. 2, pt. 3, p. 242, 1843. 1 species. Type, Mrusca longipes Fabricius.

I Caricea Desvordy, Essai Myod., p. 530, 1830. 18 supposed species. Type, Musca tigrina Fabricius (the supposed species 2 to 7 ), by designation of RoNDAxi, Dipt. Ital. Prodr., vol. 1, 1856, p. 98. Equals Coenosia MIEIGen, 1826.

$\int$ Carlottrmyia. Bigot, Bull. Soc. Ent. France for 1877, p. 26, 1877. 1 species. Type, Diacrita costalis Gersteck ER (as mœrens, new species). Equals Diacrita Gerst.eck ER, 1860 .

$\checkmark$ Carpomya Rondani, Dipt. Ital. Prodr., vol. 1, p. 111, 1856. 1 species. Type, Musca tussilaginis FABricrus (as arctii De GeER). Equals Terellia Desvordy, 1830.

$\checkmark$ Cassidæmyia Macqtart, Hist. Nat., Dipt., vol. 2, p. 162, 1835. 9 species. Type, Tachina gagatina MeIGen, by designation of Westwood, Intr., vol. 2, Synops., 1840, p. 139. Equals Rhinophora Desvordy, 1830.

J Catabomba Osten Sacken, Bull. 3, U. S. Geol, Surv., p. 326, 1877. 1 species. / Type, Musca pyrastri Linneus. Equals Scæva Fabricius, 1805.

Catachrta Brauer and Bergenstam, Denkschr. Kais. Akad. Wiss. Wien, vol. 58, p. 329, 1891. 1 species. Type, Catachæta depressariæ Brauer and Bergenstamm. Equals Phryxe Desvoidy, 1830.

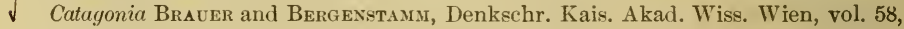
p. 348, 1891. 1 species. Type, Exorista aberrans Rondani (as nemestrina, new species). Equals Winthemia Desvordy, 1830. 
Catemophrys Townsend, Smiths. Misc. Coll., vol. 51, p. 65, 1908. 1 species. Type, Tanderwulpia sequens Townsend. Equals Vanderwulpia Townsend, 1891.

$\mathcal{C}$ Cecidogona Loew, Ent. Zeit. Stettin, vol. 5, p. 324, 1844. 1 species. Type, Lestremia carnea Loew. Equals Lestremia MAcquart, 1826.

$\mathcal{J}$ Cecidomyia MeIGen, Illiger's Mag., vol. 2, p. 261, 1803. 1 species. Type, Tipula pini De Geer. Equals Itonida MeIG EN, 1800.

Celatoria Coquilletr, Ins. Life, vol. 2, p. 235, 1891. 1 species. Type, Melanophora diabroticx SHIMer (as crawii, new species).

$\mathcal{J}$ Celeripes Montagu, Trans. Linn. Soc. Lond., vol. 11, p. 11, 1815. 1 species. Type, Pediculus vespertilionis Linnæus. Equals Nycteribia Latreille, 1796.

$\checkmark$ Cellia Theobald, Journ. Trop. Med., vol. 5, p. 183, June 16, 1902. 1 species. Type, Anopheles pharoensis Theobald. Syn., Laverania Theobald, June 16, 1902; Nyssorhynchus Blanchard, July 4, 1902; Arribalzagia Theobald, 1903.

Cenosoma Wulp, Biol. Centr.-Amer., Dipt., vol. 2, p. 166,1890. 1 species. Type, Cenosoma signifera Wulp. Equals Ormia Desvordy, 1830.

$\checkmark$ Centor Loew, Zeitschr. Ent. Breslau, vol. 15, p. 7, 1866.* 3 species. Type, Oscinis cereris Fallen, the first species, by present designation. Not Centor Schönherr, 1847. Equals Cetema Handel, 1907.

$\checkmark$ Centrocera Pokorny, Verh. Zool.-Bot. Ges. Wien, vol. 43, p. 537, 1893. 1 species. Type, Musca pedella Fallen (as Cenosia decipiens Meigen). Equals Coenosia Meigen, 1826.

$\checkmark$ Cephalemyia Latreille, Nouv. Dict. Hist. Nat., vol. 23, p. 273, 1818. 1 species. Type, Estrus ovis Linnæus. Equals Oestrus Linnæus, 1758.

Cephalia Meigen, Syst. Beschr. Zweifl. Ins., vol. 5, p. 293, 1826. 1 species (as 2). Type, Cephalia rufipes Meigen. Syn., Myrmecomya Desvoidy, 1830.

Cephalops Falden, Spec. Ent. Dipt. Exh., p. 10, 1810. 1 species. Type, Cephalops æneus Fallen. Equals Dorilas Meigen, 1800.

$\checkmark$ Cephenemyia Latreille, Nouv. Dict. Hist. Nat., vol. 23, p. 271, 1818. 1 species. Type, Oestrus trompe Linnæus. Syn., Endocephala Lioy, 1865.

$\checkmark$ Cephenus. Berthold, Nat. Fam. Thier., p. 506, 1827. Change of name for Systropus Wiedemank, 1820 (misspelled Systrophus), under the mistaken impression that it is preoccupied by Systropha Illiger, 1806. Type, Systropus macilentus Wiedemann. Equals Systropus WiedemanN, 1820.

$\checkmark$ Cephysa Desvordy, Hist. Nat. Dipt., vol. 2, p.677, 1863. 1 species. Type, Musca rudis Fabricius (as muscidea, new species). Equals Pollenia Desvoidy, 1830.

Cerajocera Rondani, Dipt. Ital. Prodr., vol. 1, p. 111, 1856. 1 species. Type, Musca cornuta Fabricius. Equals Terellia Desvoidy, 1830.

$\checkmark$ Ceranthia Desvoidy, Essai Myod., p. 88, 1830. 1 species (as 2). Type, Ceranthia fulvipes Desvoidy. Syn., Ceromya Desvoidy, 1830; Cænis Desvoidy, 1853; Vafrellia Desvoidy, 1863; Cerophora Desvoidy, 1863; Talmonia Desvoidy, 1863; Lythia DesyoIDy, 1863; Entomophaga Liox, 1864.

Ceratitis MacLeay, Zool. Journ., vol. 4, p. 482, 1829. 1 species. Type, Trypeta capitata Wiedemann (as citriperda, new species). Syn., Petalophora Macruart, 1835; Halterophora Rondani, 1861.

$\checkmark$ Ceratobarys Coquilletr, Journ. N. Y. Ent. Soc, vol. 6, p. 45, 1898. 1 species. Type, Hippelates eulophus LoEw.

$\checkmark$ Ceratochrta Brauer and Bergenstamm, Denkschr. Kais. Akad.Wiss. Wien, vol. 56, p. 92, 1889. 1 species. Type, Ceratochæita prima Brader and Bergenstamm. Equals Phryxe Desvoidy, 1830.

Ceratocystia Dyar and KNAB, Journ. N.Y. Ent. Soc., vol.14, p. 183, 1906. 1 species. Type, Culex discolor Coquilletr. Equals Grabhamia Theobald, July 25, 1903.

$\checkmark$ Ceratolophus Kiefrer, Bull. Soc. Ent. France for 1899, p. 69, 1899. 1 species. Type, Ceratopogon femorata Meigen. Equals Serromyia Meigen, 1818. 
$\checkmark$ Ceratomyiella Townsend, Trans. Amer. Ent. Soc., vol. 18, p. 379, 1891. 1 species. Type, Ceratomyiella conica Townsend. Equals Paradidyma Brauer and BergexSTAMM, 1891.

$\mathcal{J}$ Ceratomyza Schiner, Wien. Ent. Monatschr., vol. 6, p. 434, 1862. Change of name for Odontocera Macquart, 1835, not of Serville, 1833. Type, Chlorops denticornis Panzer. Equals Cerodontha Rondani, 1861.

$\checkmark$ Ceratophya Wiedemann, Anal. Ent., p. 14, 1824.* 2 species. Type, Ceratophya notata Wiedemann, the first species, by present designation. Equals Microdon Meigen, 1803.

$J$ Ceratopogan Meigen, Illiger's Mag., vol. 2, p. 261, 1803. 1 species. Type, Ceratopogon communis Meigen (as Tipula barbicornis Fabricius). Equals Helea Meigen, 1800.

$\checkmark$ Ceratoxys Rondani, Dipt. Ital. Prodr., vol. 4, p. 10, 1861 . Change of name for Meckelia Desvoidy, 1830, not of Leuckart, 1828. Type, Musca hortulana Rossi. Equals Ceroxys Macquart, 1835.

$\checkmark$ Ceraturgopsis Johnson, Psyche, vol. 10, p. 111, 1903. 1 species. Type, Dasypoyon cornutus WIEDEMANN.

$\checkmark$ Ceraturgus Wiedemann, Anal. Ent., p. 12, 1824.* 1 species. Type, Dasypogon aumilentus FABRICIUS.

J Ceria Scopoli, Ent. Carn., p. 351, 1763. 2 species. Type, Tipula notata Linneus, the first species (as decemnodia, new species), by present designation. Equals Scathopse GEOFfroy, 176\%.

$\checkmark$ Ceria Fabricius, Ent. Syst., vol.4, p. 277, 1794. 1 species (without name). Type, Musca conopsoides Linnwus. Not Ceria Scopoli, 1763. Equals Cerioides Rondani, 1850.

$\checkmark$ Ceriogaster Williston, Trans. Amer. Ent. Soc., vol. 15, p. 285, 1888. 1 species. Type, Ceriogaster fascithorax WiLliston.

$\checkmark$ Cerioides Rondani, Ann. Soc. Ent. France for 1850, p. 211, 1850. 1 species. Type, Ceria subsessilis Illiger. Syn., Céria Fabricius, 1794, preoceupied; Sy hiximorpha Rondani, 1850.

$\mathcal{J}$ Cerodontha Rondani, Dipt. Ital. Prodr., vol. 4, p. 10, 1861. Change of name for Odontocera II Acquart, 1835, preoccupied. Type, Chlorops denticornis Panzer. Syn., Odoutocera Macquart, 1835, preoccupied; Ceratomyza Schiner, 1862.

$\mathcal{C}$ Ceromasia Rondani, Dipt. Ital. Prodr., vol. 1, p. 71, 1856. No species. In vol. 4, 1861 , pp. 18 and 19, 10 species. Type, Tachina rutila MeIgen, the last species, by designation of Brauer, Verh. Zool.-Bot. Ges. Wien, vol. 43, 1893, p. 476.

$\checkmark$ Ceromya Desvorny, Essai Myod., p. 86, 1830. 3 species (as 5). Type, Tachina bicolor MeIcien, 'the fourth supposed species (as testacea, new species), by present designation. Equals Ceranthia Desvordy, 1830.

$\checkmark$ Cerophora Desvoidy, Hist. Nat. Dipt., vol. 1, p. 700, 1863. 1 species. Type, Cerophora funesta Desvoidy. Equals Ceranthia Desvoidy, 1830.

$\checkmark$ Ceroplatus. See Keroplatus.

J Cerotainia Schiner, Verh. Zool.-Bot. Ges. Wien, vol. 16, p. 673, 1866. 1 speecies. Type, Laphria xanthoptera WiedeMans.

$\checkmark$ Cerotelion Rondani, Dipt. Ital. Prodr., vol. 1, p. 191, 1856. 1 species. Type, Tipula lineata Fabricius (as Platyura laticornis Meigev). Equals Keroplatus Bosc, 1792.

$\checkmark$ Ceróxys Macquart, Hist. Nat. Dipt., vol. 2, p. 437, 1835. 8 species. Type, Musca urticx Linnæus, the second species, by designation of Westwood, Intr., vol. 2, Synops., p. 149, 1840. Syn., Meckelia Desvordy, 1830, preoceupied; Ceratoxys RosDANI, 1861; Anacampta Loew, 1868.

$\mathcal{J}$ Cestonia Rondani, Dipt. Ital. Prodr., vol. 4, p. 105, 1861. 1 species. Type,Cestonia cineraria RondanI.

$\checkmark$ Cetema Hendel, Wien. Ent. Zeit., vol. 26, p. 98, 1907. Change of name for Centor Loew, 1866, not Schönherr, 1847. Type, Osciniscereris Fallen. Syn., Centor Leew, 1866, preoccupied. 
Chætoclusia Coquillett, Proc. Ent. Soc. Washington, vol. 6, p. 93, 1904. 1 species. Type, Chrotoclusia bakeri Coquillett.

Chætocœlia Giglio-Tos, Boll. Mus. Zool. Univ. Torino, vol. 8, No. 158, p. 11, 1893. 1 species. Type, Chrtocelia palans Giglio-Tos.

Chætogædia Brauer and Bergenstamm, Denkschr. Käis. Akad.Wiss. Wien, vol. 58, p. 336, 1891. 2 species. Type, Prospherysa vilis WulP, the first species, by designation of Townsend, Smiths. Misc. Coll., vol. 51, 1908, p. 94. Syn., Phrissopolia TownSEND, 1908.

Chætoglossa Townsend, Trans. Amer. Ent. Soc., vol. 19, p. 125, 1892. 2 species (as 3). Type, Chætoglossa picticornis Townsend, the first species, by original designation. Chætona Wulp, Biol. Centr.-Amer., Dipt., vol. 2, p. 253, 1891. 2 species. Type, Dexia longiseta WIEDEManN, the first species, by present designation.

$\checkmark$ Chrtopeleteria M1к, Wien. Ent. Zeit., vol. 13, p. 100, 1894. 1 species. Type, Echinomyia popelii Portschinsky. Equals Peleteria Desvoidy, 1830.

$\checkmark$ Chætophleps Coquillett, Journ. N. Y. Ent. Soc., vol. 3, p. 51, 1895. 1 species. Type, Chaetophleps setosa Coquillett.

Chætoplagia Coquillett, Journ. N. Y. Ent. Soc., vol. 3, p. 98, 1895. 1 species. Type, Chrtoplagia atripennis Coquilletт.

Chætopsis Loew, Berliner Ent. Zeitschr., vol. 11, p. 315, 1868. 2 species. Type, Ortalis ænea Wiedeman, the first species, by present designation.

$\checkmark$ Chætosa Coquillett, Journ. N.Y. Ent. Soc., vol. 6, p. 163, 1898. 1 species. Type, Cordilura punctipes MEIG EN.

$\checkmark$ Chrtotachina Brauer and Bergenstamm, Denkschr. Kais. Akad. Wiss. Wien, vol. 56 , p. 98, 1889. 1 species. Type, Tachina simulans Meigen (as rustica Meigen). Equals Exorista Meigen, 1803.

$\mathcal{J}$ Chalarus Walker, Ent. Mag., vol. 2, p. 269, 1834. 1 species (as 2). Type, Cephalops spurius Fallen. Syn., Atelenevra Macquart, 1834.

$\checkmark$ Chalcidimorpha Westwood, Ann. Soc. Ent. France for 1835, p. 685, 1835. 4 species. Type, Chalcidimorpha fulvipes W EsTwoon, the first species, by designation of Macquart, Dipt. Exot., vol. 1, pt. 2, 1838, p. 153 . Equals Xenomyza WiedeMANN, 1817.

Chalcomyia Williston, Bull. Brooklyn Ent. Soc., vol. 7, p. 133, 1885.1 species. Type, Myolepta ærea Loew.

Chamæmyia Meigen, Illiger's Mag., vol. 2, p. 278, 1803. No species. In his Syst. Beschr., vol. 6, p. 93, 1830, he mentioned 1 species. Type, Chamæmyia elegans Pan7er. Syn., Ochtiphila Falden, 1823; Estelia Desvoidy, 1830.

Chamæsyrphus Mıк, Wien. Ent. Zeit., vol. 14, p. 133, 1895. 1 species. Type. Rhingia scævoides FALLEN.

$\checkmark$ Chaoborus Lichtenstein, Arch. Zool. (Wiedemann's), vol. 1, p. 174, 1800.1 species. Type, Tipula crystallina De GeEr (as antisepticus, new species). Syn., SAyomyia CoquILleTt, 1903.

Charadrella Wulp, Biol. Centr.-Amer., Dipt., vol. 2, p. 341, 1896. 1 species. Type, Charadrella macrosoma WuLP.

Chasmatonotus Loew, Berliner Ent. Zeitschr., vol. 8, p. 51, 1864. 1 species. Type, Chasmatonotus unimaculatus LoEw.

Chauna Loew, Ent. Zeit. Stettin, vol. 8, p. 370, 1847. 1 species. Type, Chauna variabilis Lòw. Not Chauna Illiger, 1811. Equals Neochauna Williston, 1896.

Cheilopogon Rondani, Dipt. Ital. Prodr., vol. 1, p. 157, 1856. 1 species. Type, Asilus diadema Fabricius (as Dasypogon punctatus Fabricius). Equals Dasypogon MeIGEN, 1803.

Cheilosia Meigen, Syst. Beschr. Zweifl. Ins., vol. 3, p. 289, 1822.1 species. Type, Syrphus flavipes Panzer (as depila, new species).

Chelifera Macquart, Monogr. Empides, p. 150, 1823. 1 species. Type, Tachydromia precatoria Fallen (as raptor, new species). Syn., Mantipeza Rondani, 1856; Polydromya Bigot, 1857. 
Cheligaster Macquart, Hist. Nat., Dipt., vol. 2, p. 479, 1835. 3 species. Type, Musca putris Linnaus, the first species, by present designation. Equals Themira Desvoidy, 1830.

Chelipoda Maceuart, Monogr. Empides, p. 148, 1823. 2 species. Type, Empis melanocephala Fabricius, the second species, by designation of Coquilletr, Proc. Ent. Soc. Washington, vol. 5, 1903, p. 247. Equals Hemerodromia Meigen, 1822.

$\checkmark$ Chemalida Rondanl, Dipt. Ital. Prodr., vol. 1, p. 180, 1856. 1 species. Type, Erioptera tænionota Meigen. Equals Polymeda Meigen, 1800.

$\checkmark$ Chenesia Macquart, Hist. Nat., Dipt., vol. 1, p. 151, 1834. 1 species. Type, Orphnephila devia Haliday (as testacea, new species). Equals Orphnephila Haliday, 1831.

$\checkmark \quad$ Chersodromia WALKer, List Dipt. Brit. Mus., vol. 4, p. 1157, 1849. 2 species. Type, Tachypeza arenaria HALIDAY (as brevipennis Zetterstedt).

$\checkmark$ Chetocera Desvoidy, Ėssai Myod., p. 697, 1830. 1 species. Type, Sciomyza albocostata Fallen (as claripennis, new species). Syn., Melina Desvoidy, 1830; Arina Desvoidy, 1830.

$\checkmark$ Chetogena Rondani, Dipt. Ital. Prodr., vol. 1, p. 68, 1856. 1 species. Type, Tachina assimilis Fallen (as gramma Meigen). Equals Phorocera Desvordy, 1830.

- Chetoliga Rondani, Dipt. Ital. Prodr., vol. 1, p. 66, 1856. 1 species.' Type, Tachina gnava Meigen. Equals Carcelia Desvordy, 1830.

$\checkmark$ Chiastocheta Pokorny, Verh. Zool.-Bot. Ges. Wien, vol. 39, p. 568, 1889.1 species. Type, Aricia trollii Zetterstedt. Equals Chirosia Rondani, 1856.

Chionea Dalman, Kongl. Vet. Acad. Handl. for 1816, p. 104, 1816. 1 species. Type, Chionea araneoides Dalman.

$\checkmark$ Chiromyza Wiedemann, Nova Dipt. Gen., p. 20, 1820** 2 species. Type, Chiromyza vittata Wiedemann, the first species, by designation of BraUer, Denkschr. Kais. Akad. Wiss. Wien, vol. 44, p. 86, 1882.

$\checkmark \cdot$ Chironomus Meigen, Illiger's Mag., vol. 2, p. 260, 1803. 3 species. Type, Tipula plumosa Linneus, the first species, by designation of Latreille, Consider. Général., p. 442, 1810. Equals Tendipes MeIGen, 1800.

$\checkmark$ Chirosia Rondani, Dipt. Ital. Prodr., vol. 1, p. 102, 1856. 1 species. Type, Aricia albitarsis Zetterstedt. Syn., Chiastocheta Pokorny, 1889; Rhadina Kowarz, 1893.

$\checkmark$ Chloria Schiner, Wien. Ent. Monatschr., vol. 6, p. 151, 1862. 1 species. Type, Musca demandata Fabricius. Equals Physiphora Fallen, 1810.

$\checkmark$ Chlorina Desvordy, Essai Myod., p. 603, 1830. 2 species. Type, Chlorina thoracica Desvordy, the first species, by present designation. Equals Pegomya DesvolDy, 1830.

$\checkmark$ Chloromyia Duncan, Mag. Zool. and Bot., vol. 1, p. 164, 1837. 3 species. Type, Musca formosa Scopoli, the first species, by present designation.

Chloropisca LoEw, Zeitschr. Ent. Breslau, vol. 15, p. 79, 1866.* 5 species. Type, Chlorops glaora Meigen, the third species, by present designation.

$\checkmark$ Chloroprocta Wulp, Biol. Centr.-Amer., Dipt., vol. 2, p. 296, 1896. 1 species. Type, Chloroprocta semiviridis WulP.

$J$ Chlorons Meigen, Illiger's Mag., vol. 2,:p. 278, 1803. No species. In his Syst. Beschr., vol. 6, 1830, pp. 140 to 163,60 species. Type, Chlorops lata Mieigen, the sixth species, by designation of Rondani, Dipt. Ital. Prodr., vol. 1, 1856, p. 125. Equals Titania MeIgen, 1800.

$\checkmark$ Chlorosia Rondani, Dipt. Ital. Prodr., vol. 4, p. 11, 1861. Change of name for Clorisoma Rondani, 1856, under the erroneous impression that it is preoccupied by Chlorisoma Swainson, 1837. Type, Musca parvula Harris. Equals Chrysomyia MaCQUaRT, 1834.

$\checkmark$ Cholomyia Bigot, Bull. Soc. Ent. France for 1884, p. 37, 1884 . 1 species. Type, Cholomyia inæquipes Bı̈oт.

$\checkmark$ Chordonota Gerst fcker, Linn. Ent., vol. 11, p. 311, 1857. 1 species. Type, Cyphomyia inermis WIEDEMANN. 
Choristomma Stein, Berliner Ent. Zeitschr., vol. 40, p. 138, 1395. 1 species. Type, Platyccenosia mikii Strobl (as pokornyi, new species). Equals Platycœnosia Strobl, 1894.

Choristoneura RÜBSAAMEN, Berliner Ent. Zeitschr., vol. 37, p. 342, 1892. 1 species. Type, Lasioptera obtusa Loew.

Chortophila Macquart, Hist. Nat., Dipt., vol. 2, p. 323, 1835. 22 species. Type, Anthomyia sepia Meigen, the fifteenth species, by designation of Westwood, Intr., vol. 2, Synops., 1840, p. 142 . Equals Pegomya Desvoidy, 1830.

Chremia Desvordy, Hist. Nat. Dipt., vol. 2, p. 335, 1863.1 specics. Type, Tachina incinis Fallen (as ciligera, new species). Equals Leskia Desvordy, 1830.

Chromatomyia HARDY, Ann. Mag. Nat. Hist., ser. 3, vol. 4, p. 390, 1849. 7 species. Type, Phytomyza obscurella Fallen, the third species, by present designation. Equals Phytomyza Fallen, 1810.

Chrysanthrax Osten SAcken, Biol. Centr.-Amer., Dipt., vol. 1, p. 121, 1886.4 species. Type, Anthrax fulvohirta Wiedemand, the fourth species, by present designation. Equals Villa Lioy, 1864.

Chryseria Desvoidy, Hist. Nat. Dipt., vol. 2, p. 288, 1863. 2 species. Type, Musca helluo Fabricius (as gentilis, new species), the second species, by present designation. Equáls Eliozeta Rondani, 1856.

Chrysoceria Williston, Journ. N. Y. Ent. Soc., vol. 15, p. 1; 1907. 1 species. Type, Laparus pictitarsis Bigot.

Chrysochlora Latreille, in Cuvier's Regne Anim., new ed., vol. 5, p. 486, 1829. 1 species. Type, Sargus amethystinus Fabricius.

Chrysochroma Williston, Man. N. Amer. Dipt., p. 47, 1896 . Change of name for Chrysonotus Loew, 1855, not of Swainson, 1837. Type, Musca bipunctata Scopoli. Syn., Chrysonotus Loww, 1855, preoccupied; Chrysonotomyia Hunter, 1900.

Chrysoclamis Rondani, in Walker's Ins. Britt., Dipt., vol. 1, p. 279, 1851. Change of name for Ferdinandea RoNDANI, 1844, because the latter name had not been adopted by the other entomologists. Type, Conops cupreus Scopoli. Equals Ferdinandea RoNDANI, 1844.

Chrysegaster Meigen, Nouv. Class. Mouch., p. 32, 1800.* No species. In Illiger's Mag., vol. 2, p. 274, 1803, 3 species. Type, Eristalis solstitialis Fallen, the first species (as comiteriomum Fabricius), by designation of Zetrenstedt, Dipt. Scand., vol. 2, 1843, p: 816. Sỳn.; Orthonevra MACQuakr, 1829; Campineura Rondani, 1856; Melanogaster Rondani, 1857; Lejogaster Rondani, 1857; Cryptineura Вigot, 1859.

[Chrysomya Desvoidy, Essai Myod., p. 444, 1830. 22 supposed species. Type, Chrysomya regalis Desvoidy, the fourteenth species, by designation of Rondani, Arch. Zool. Modena, vol. 3, 1864, p. 27. Syn., Pycnosoma Brauer and Bergenstamm, 1894; Paracompsomyia Hough, 1898. Not American.] (Chrysomya of authors equals Paralucilia Brauer and Bergenstamm.)

Chrysomyia Macquart, Hist. Nat., Dipt., vol. 1, p. 262, 1834. 5 species. Type, Musca polita Linnæus, the third species, by designation of Westwood, Intr., vol. 2, Synops., 1840, p. 130. Syn., Microchrysa Loew, 1855; Clorisoma Rondani, 1856; Myochrysa Rondani, 1861; Chlorosia Rondani, 1861.

Chrysomyza Fallen, Scen. et Conop. Sveciae, p. 3, 1817. 1 species. Type, Musca demandata Fabricius (as splendida, new species). Equals Physiphora Fallen, 1810.

Chrysonstomyia Hunter, Trans. Amer. Ent. Soc., vol. 27, p. 124, 1900. Change of name for Chrysonotus Loew, 1855, not of Swainson, 1837. Type, Musca bipunctata Scopoli. Equals Chrysochroma WiLliston, 1896.

Chrysonotus Loew, Verh. Zool.-Bot. Ges. Wien, vol. 5, p. 146, 1855. 1 species. Type, Musca bipunctata Scopoli. Not Chrysonotus Swainson, 1837. Equals Chrysochroma Williston, 1896.

$\checkmark$ Chrysopilus Macquart, Mem. Soc. Sci. Lille for 1826, p. 403, 1826. 3 species. Type, Musca diadema Linnæus, the third species, by designation of Westwood, Intr., vol. 2, Synops., 1840, p. 134. Equals Styrex Scopoli: 1763. 
Chrysops Meigen, Nouv. Class. Mouch., p. 23, 1800.* No species. In Illiger's Mag., vol. 2, 1803, p. 267, 1 species. Type, Tubanus creutiens Livisus.

$\checkmark$ Chrysotimus Loew, Neue Beitr., vol. 5, p. 48, 1857. No species. In vol. 8, p. 74, 1861, 2 species. Type, Chrysotimus pusio LoEw, the first species, by present designation.

$J$ Chrusatorigen, Illiger's Mag., vol. 2, p. 275, 1803. 2 species. Type, Musca bicincta Linnsus, the second species, by designation of Latreille, Consid. Général., 1810, p. 443. Equals Antiopa Meigen, 1800.

$\checkmark$ Chrysotus Meigen, Syst. Beschr. Zweifl. Ins., vol. 4, p. 40, 1824. 8 species. Type, Musca nigripes Fabricius, the sixth species, by designation of Westwood, Intr., vol. 2 , Synops., 1840 , p. 134.

Chrysozona Meigen, Nouv. Class. Mouch., p. 23, 1800** No species. Hæmatopota Meigen, 1803, is a change of name. Type, Tabanus pluvialis Linnsus. Syn., Hirmatopota Meigen, 1803.

$\checkmark$ Chyliza Fallen, Opomyz. Sveciae, p. 6, 1820. 2 species. Type, Musca leptogaster Panzer, the second species, by Meigen, who, in his Syst. Beschr., vol. 5, 1826, p. 370, selected the first species as type of a new genus, Lissa. Syn., Dasyna Desvoidy, 1830.

$\checkmark$ Chymophila Macquart, Hist. Nat., Dipt., vol. 1, p. 485, 1834. 1 species. Type, Nicrodon aurifex Wiedemann (as splendens, new species). Equals Microdon Meigen, 1803.

Chyromantis Rondanl, Dipt. Ital. Prodr., vol. 1, p. 148, 1856. 1 species. Type, Tuchydromia vocatoria Fallen. Syn., Litanomyia Melander, 1902.

Chyromya Desvoldy, Essai Myod., p. 621, 1830. 1 species. Type, Mfusca flava Linneus (as fenestrarum, new species). Syn., Lisella Desvoidy, 1830; Scyphella DesvoIDY, 1830; Thyrimyza ZeTterstedt, 1847.

Cimbometopic Lioy, Atti Inst. Veneto, ser. 3, vol. 9, p. 1114, 1864. 1 species. Type, Borborus stercorarius Meigex. Equals Leptocera Olivier, 1813.

$\checkmark$ Cincticornia Felt, 23d Rep. State Ent. N. Y., p. 379, 1908. 15 species. Type, Asphondylia transersa Felt, the first species, by original designation.

$\mathcal{J}$ Cinxia Melgen, Nouv. Class. Mouch., p. 35, 1800.* No species. Sericomyia Meigen, 1803, was a change of name. Type, Musca lappona Linneus. Syn., Sericomyia Meigen, 1803.

Cistogaster Latreille, in Cuvier's Regne Anim., new ed., vol. 5, p. 511, 1829. No species. Macquart; Ins. Dipt. Nord France, Ather., 1834, pp. 74 and 75, refers three supposed species to this genus. Type, Musca globosa F ABricius, the first species, by designation of Blancirakd, Hist. Nat. Ins., vol. 3, 1840, p. 612. Syn., Pallasia Desvoidy, 1830; Gymnoclytia Brauer and Bergenstamu, 1893.

$\checkmark$ Cladochæta Coquilletr, Proc. U. S. Nat. Mus., vol. 22, p. 263, 1900. 1 species. Type, Cladochata nebulosa CoquilletT.

$\checkmark$ Cladura Osten Sacken, Proc. Acad. Nat. Sci. Phila. for 1859, p. 229, 1860. 1 species. Type, Cladura flaroferruginea Osten Sacken.

$\checkmark$ Clanis Walker, Ins. Saund., Dipt., p. 9, 1850. 18 species. Type, Pangonius lasiophthalmus MACQUART, the first species (as contigua WALKER), by present designation. Equals Pangonius Latreille, 1802.

Clasiopa Stenhammar, Kongl. Vet. Acad. Handl. for 1843, p. 159, 1844. 1 species. Type, Notiphila obscurella Faldex. Equals Discocerina Macquart, 1835.

Clausicella Rondani, Dipt. Ital. Prodr., vol. 1, p. 61, 1856. 1 species. Type, Clausicella suturata Rondani. Syn., Istoglossa Rondani, 1856.

$\checkmark$ Clelia Desvoidy, Essai Myod., p. 255, 1830. 2 species (as 4). Type, Musca aterrima Villiers, the first and last supposed species (as agilis and erythrocera, new species), by designation of Desvordy, Hist. Nat. Dipt., vol. 2, 1863, p. 61. Equals Leucostoma Meigen, 1803.

$\checkmark$ Clemelis Desvoidy, Hist. Nat. Dipt., vol. 1, p. 481, 1863. 1 species (as 6). Type, Musca libatrix Panzer. Equals Zenilla Desvoidy, 1830. 
Cleodora Desvoldy, Hist. Nat. Dipt., vol. 1, p. 1047, 1863.1 species. Type, Tachina simulans Meigen (as ancilla, new species). Equals Exorista Meigen, 1803.

Cleona Mergen, Nouv. Class. Mouch., p. 30, 1800* No species. Callomyia MeIGEN, 1804, is a change of name. Type, Callomyia elegans Meigen. Syn., Callomyia Meigen, 1804; Heteroneura Fallen, 1810; Callomyza Fallen, 1815.

Cleonice Desvoldy, Hist. Nat. Dipt., vol. 1, p. 1097, 1863. 1 species. Type, Tachina grisea Fallen. Equals Macquartia Desvoldy, 1830.

Clinocera Meigen, Illiger's Mag., vol. 2, p. 271, 1803. No species. In 1804, 1 species. Type, Clinocera nigra Meigen. Equals Atalanta Meigen, 1800.

Clinodiplosis Kieffer, Bull. Soc. Ent. France for 1894, p. 280, 1894. 1 species. Type, Diplosis cilicrus KIEFFER.

Clinogaster Wulp, Tijdschr. Ent., vol. 35, p. 189, 1892. 1 species. Type, Clinogaster notabilis WULP.

Clinoneura Brauer and Bergenstamm, Denkschr. Kais. Akad. Wiss. Wien, vol. 56, p. 119, 1889. 1 species. Type, Estheriatibialis Desvoldy (as Dexia rubriventris MacQUaRT). Equals Phorostoma Desvoidy, 1830.

Clinopera Wulp, Biol. Centr.-Amer., Dipt., vol. 2, p. 305, 1896. 9 species. Type, Clinopera hieroglyphica WulP, the third species, by present designation. Equals Cyrtoneurina Giglio-Tos, 1893.

Clinorhyncha Loew, Dipt. Beitr., vol. 4, p. 21, 1850. 1 species. Type, Clinorhynch a chrysanthemi Loew.

Clista Meigen, Syst. Beschr. Zweifl. Ins., vol. 7, p. 208, 1838. 7 species. Type, Tachina merens Meigen, the second species, by designation of Rondani, Dipt. Ital. Prodr., vol. 1, 1856, p. 76. Equals Rhinophora DesvoIdy, 1830.

Clistomorpha Townsend, Can. Ent., vol. 24, p. 79, 1892. 1 species. Type, Xysta didyma Loww (as hyalomoides, new species). Equals Eliozeta Rondanl, 1856.

[Clitellaria Meigen, Illiger's Mag., vol. 2, p. 265, 1803. 1 species. Type, Stratiomys ephippium Fabricius. Equals Potamida Melgen, 1800. Not American.] (Clitellaria of authors equals Adoxómyia Kertē̃z.) $)^{-}$.

Clorisoma Rondan1, Dipt. Ital. Prodr., vol. 1, p. 168, 1856. 1 species. Type, Musca parvila Harris (as Sargus pallipes Meigen). Equals Chrysomyia Macquart, 1834.

Clusia Haliday, Ann. Nat. Hist., vol. 2, p. 188, Nov., 1838. 1 species. Type, Heteromyza flava Meigen. Syn., Macrochira Zettenstedt, 1838; Peratochetus Rondani, 1856; Stomphastica Loew, 1864.

Clusiodes Coquillett, Proc. Ent. Soc. Washington, vol. 6, p. 93, 1904. Change of name for Heteroneura FALLen, 1823, not of FALLEN, 1810. Type, Heteroneura albimana Meigen. Syn., Heteroneura Fallen, 1823, preoceupied.

Clythia Meigen, Nouv. Class. Mouch., p. 30, 1800.* No species. Platypeza Meigen, 1803, is a change of name. Type, Platypeza fasciata Mrigen. Syn., Platypeza Meigen, 1803; Calotarsa Townsend, 1894.

Clytia Desvoldy, Essai Myod., p. 287, 1830. 5 species (as 7). Type, Musca continua PANzer, the last three supposed species, by designation of W-ESTwood, Intr., vol. 2, Synops., 1840, p. 139. Not Clytia Lamarck, 1812. Equals Eliozeta Rondani, 1856.

Clytiomya Rondani, Dipt. Ital. Prodr., vol. 4, p. 9, 1861. Change of name for Clytia Desvoldy, preoccupied. Type, Musca continua Panzer. Equals Eliozeta Rondanl, 1856.

Cnemacantha Macquart, Hist. Nat., Dipt., vol. 2, p. 586, 1835.1 species. Type, Heteroneura muscaria Fallen. Equals Sapromyza Fallen, 1810.

Cnephalia Rondani, Dipt. Ital. Prodr., vol. 1, p. 62, 1856. 1 species. Type, Tachina \&ucephala Meigen (as Gonia hebes Meigen). Equals Spallanzania Desvoidy, 1830.

Cnephaliodes Brauer and Bergenstamm, Denkschr. Kais. Akad. Wiss. Wien, vol. 58, p. 253, 1891. 1 species. Type, Cnephaliodes perversus Brauer and Bergenstamm. Equals Larvævora Meigen, 1800. 
Cnephaotachina Brauer and Bergenstamu, Denkschr. Kais. Akad. Wiss. Wien, vol. 61, p. 612, 1894. 1 species. Type, Cnephaotachina crepusculi Brauer and BerGenstamm. Syn., Eudora Desvoidy, 1863, preoceupied; Eudoromyia Bezz1, 1906.

$\checkmark$ Coelodiazesis Dyar and KNAB, Journ. N. Y. Jint. Soc., vol. 14, p. 177, 1906.1 species. Type, Anopheles barberi Coquilletr. Equals Anopheles Meigen, 1818.

$\checkmark \quad$ Cœloglutus Aldrich, Trans. Ent. Soc. Lond. for 1896, p. 338, 1896.1 species. Type, Cologlutus concavus AlDrich.

$\checkmark$ Cœlomyia Haliday, in Westwood's Intr., vol. 2, Synops., p. 143, 1840.1 species. Type, Colomyia mollissima HaLIDAY.

$\checkmark$ Cœlopa Melgen, Syst. Beschr. Zweifl. Ins., vol. 6, p. 8, 1830. 1 species. Type, Colopa pilipes Halidar (as Musca frigida Fabricius). Syn., Fucomyia Halıdy, 1838.

- Colosia Winnertz, Verh. Zool.-Bot. Ges. Wien, vol. 13, p. 796, 1863.2 species. Type, Boletina flava STEgER, the first species, by designation of Johannsen in Wytsman's Gen. Ins.; Dipt., Mycet., 1909, p. 86.

$\checkmark \quad$ Cœnia Desvordy, Essai Myod., p. 800, 1830. 1 species. Type, Ephydra palustris FALLEN (as caricicola, new species).

- Cœnomyia Latrielle, Precis, p. 159, 1796. No species. In his Hist. Nat. Crust. et Ins., vol. 3, p. 439, 1802, 1 species. Type, Musca ferruginea Scopour.

$\checkmark$ Canosia Melgen, Syst. Beschr. Zweifl. Ins., vol. 5, p. 210, 1826. 28 species. Type, Musca geniculata FAllen, the twentieth species, by designation of Rondani, Atti Soc. Ital. Sci. Nat., vol. 9, 1866, p. 9. Syn., Caricea Desvordy, 1830; Limosia Desvoidy, 1830; Palusia Desvoldy, 1830;? Eriostyla Lıoy, 1864; Centrocera Pokorny, 1893; Limnospila SchNaBL, 1902.

Coilometopia Macquart, Dipt. Exot., Suppl. 2, p. 91, 1847. 1 species. Type, Scatophaga trimaculata FABRICIUs (as ferruginea, new species).

$\checkmark$ Coloboneura Melander, Trans. Amer. Ent. Soc., vol. 28, p. 229, 1902.1 species, Type, Coloboneura imusituta MeLAN DER.

$\checkmark$ Colpodia Winnertz, Linn. Ent., vol. 8, p. 188, 1853.1 species. Type, Colpodia angustipennis WINNERTZ.

$\checkmark$ Comasies Osten Sacken, Bull. 3, U. S. Geol. Surv., p. 256, 1877.1 species. Type, Comastes robusius Osten Sacken. Equals Heterostylum Macquart, 1848.

$\checkmark$ Comatacta Coquilletr, Can. Ent.., vol. 34, p. 199, 1902. 1 species. Type, Brachycoma pallidula WuLP.

$\checkmark$ Commoptera Brues, Amer. Nat., vol. 35, p. 344, 1901. 1 species. Type, Commoptera solenopsidia BRues.

Compsilura Bouché, Nat. Ins., p. 58, 1834. 2 species (as 3). Type, Tachina concinnata Meigen, the first species, by present designation. Syn., Doria Meigen, 1838; Machirixa Rondani, 1859.

$\mathcal{J}$ Compsomyia Rondani, Ann. Mus. Civ. Stor. Nat. Genova, vol. 7, p. 425, 1875.4 species. Type, Musca dux Eschscholz, the first species, by present designation. Equals Calliphora Desroidy, 1830.

$\checkmark$ Comyops Wulp, Biol. Centr.-Amer., Dipt., vol. 2, p. 262, 1891.2 species. Type, Comyops nigripennis Wulp, by designation of Brauer and Bergenstamm, Denkschr. Kais. Akad. Wiss. Wien, vol. 60, 1893, p. 183.

$\checkmark$ Cona Schellenberg, Genres Mouch. Dipt., pl. 13, figs. 1 and 2; 1803.1 species. Type, Musca fenestralis Linnæus. Equals Omphrale Meigen, 1800.

$\checkmark$ Conchyliastes Theовald, in Howard's Mosquitoes, p. 235, 1901. 2 species. Type, Culex posticatus WIEDEnann (as musicus $\mathrm{SAY}$ ), the first species, by present designation. Equals Janthinosoma ArribalzaGA, 1891.

* Condidea Coquilletr, Can. Ent., vol. 39, p. 75, 1907. 1 species. Type, Condidea lita Coquillett.

$\sqrt{ }$ Coniceps Loww, Monogr. Dipt. N. Amer., vol. 3, p. 177, 1873.1 species. Type, coniceps niger Loww. 
Conicera Meigen, Syst. Beschr. Zweifl. Ins., vol. 6, p. 226, 1830. 1 species. Type, Conicera atra MeIgev.

Conopejus Rondani, Mag. Zool., ser. 2, vol. 7, Ins., p. 7, 1845. 3 species. Type, Conops quadrifasciatus DE GeER, the first species, by original designation. Equals Conops Linneus, 1758.

Conophorus Meigen, Illiger's Mag., vol. 2, p. 268, 1803. 1 species. Type, Bombylius virescens Fabricius (as maurus Mikan). Syn., Ploas Latreille, 1805.

Conopoideus Rondanı, Mag. Zool., ser. 2, vol. 7, Ins., p. 9, 1845. 1 species. Type, Conops ferruginea Macquart. Equals Conops Linneus, 1758.

Conops Linneus, Syst. Nat., 10th ed., p. 604, 1758. 6 species. Type, Conops favipes Linneus, the fifth species, by designation of Curtis, Brit. Ent., 1831, p. 377. Syn., Conopejus Rondani, 1845; Conopoideus Rondanl, 1845; Sphyxosoma Rondani, 1856; Bombibia Lior, 1864.

Conopsida Macquart, Dipt. Exot., Suppl. 4, pt. 2, p.267, 1851. 1 species. Type, Cephalia femoralis Wiedemann. Equals Michogaster Macquart, 1835.

Contarinia Rondani, Atti Soc. Ital. Sci. Nat., vol. 2, p. 289, 1861. 1 species. Type, Tipula loti De Geer. Syn., Stictodiplosis KiefFer, 1894; Eudiplosis KiefFer, 1894.

Copecrypta Townsend, Smiths. Misc. Coll., vol. 51, p. 109, 1908. 1 species. Type, Schineria ruficauda WulP. Equals Cuphocera Macquart, 1845.

$\checkmark$ Copestylum Macquart, Dipt. Exot., Suppl. 1, p. 124, 1846. 1 species. Type, Volucella marginata $\mathrm{S}_{\mathrm{AY}}$ (as flaviventris, new species).

Cophura Osten Sacken, Biol. Centr.-Amer., Dipt., vol. 1, p. 181, 1887. 1 species. Type, Cophura sodalis Osten Sacken. Syn., Blax Loew, 1872, preoccupied; Blacodes LoEw, 1874, preoccupied; Loewiella WiLliston, 1896.

$\mathcal{J}$ Coprina Desvordy, Essai Myod., p. 810, 1830. 1 species. Type, Borborus denticulatus Meigen (as bovina, new species). Equals Cypsela Meigen, 1800.

$\mathcal{J}$ Coprina Zetterstedt, Isis von Oken for 1837, p. 35, 1837. 1 species. Type, Musca h pipiens Linneus. Equals Syritta St. Fargeau and Serville, 1828.

$\checkmark$ Coprodiplosis Kiefrer, Bull. Soc. Ent. France for 1894, p. 280, 1894. 1 species. Type, Diplosis polypori Loew. Equals Leptodiplosis KIEFFER, 1894.

$\checkmark$ Coproica Rondani, Dipt. Ital. Prodr., vol. 4, p. 10, 1861. Change of name for Heteroptera Macquart, 1835, preoccupied. Type, Copromyza pusilla Fallen. Equals Cypsela Meigen, 1800.

$\checkmark$ Copromyza Fallen, Spec. Ent. Dipt. Exh., p. 19, 1810. No species. In his Het. Sveciæ, 1820, pp. 6 to 8,7 species. Type, Copromyza equina FALLen, the second species, by designation of Zetrers'redt, Dipt. Scand., vol. 6, 1847, p. 2475. Syn., Mycetia Desvoidy, 1830; Cimbometopia Lioy, 1864; Isogaster Lioy, 1864; Fungobia Lioy, 1864.

Coquillettia Williston, Man. N. Amer. Dipt., p. 65, 1896. 1 species. Type, Spogostylum vandykei Coquillett. Not Coquillettia Uhler, 1890. Equals Anthrax Scopoli, 1763.

Coquillettidia Dyar, Proc. Ent. Soc. Washington, vol. 7, p. 45, 1905. 4 species. Type, Culex perturbans WALKER, the first species, by original designation.

Coquillettomyia FeLT, 23d Rep. State Ent. N. Y., p. 398, 1908. 3 species. Type, Mycodiplosis lobata FeLt, the first species, by original designation.

Cordilụra Fallen, Spec. Ent. Dipt. Exh., p. 15, 1810. 1 species. Type, Musca pubera Linneus. Syn., Mosina Desvoidy, 1830.

Cordylidexia Gigiıo-Tos, Boll. Reale Acad. Sci. Torino, ser. 2, vol. 44, p. 537, 1894. Change of name for Cordyligaster MACQUART, 1843, under the mistaken impression' that it is preoccupied by Cordylegaster BILlberg, 1820. Type, Dexia petiolata Wiedemann. Equals Cordyligaster Macquart, 1843.

Cordyligaster Macquart, Dipt. Exot., vol. 2, pt. 3, p. 90, 1843.1 species. Type, Dexia petiolata Wiedemann. Syn., Cordylidexia Giglio-Tos, 1894. 
$\int$ Corethra Meigen, Illiger's Mag., vol, 2, p. 260, 1803. 1 species. Type, Tipula culiciformis DE Geer. Syn., Mochlonyx Loew, 1844 .

$\checkmark$ Corethrella Coqulleter, Journ. N. Y. Ent. Soc., vol. 10, p. 191, 1902. 1 species. Type, Corethrella brakeleyi Conuillett.

$\checkmark$ Corizoneura Rondani, Arch. Zool., vol. 3, p. 85, 1864. 3 species. Type, Pangonius appendiculata MACQUART, the first species, by present designation. Equals Pangonius Latreille, 1802.

$\checkmark$ Coronimyia Townsend, Trans. Amer. Ent. Soc., vol. 19, p. 128, 1892. 1 species. Type, Coronimyia geniculata Townsend. Equals Epigrimyia Townsend, 1891.

$\checkmark$ Coryneta Melgen, Nouv. Class. Mouch., p. 27, 1,00.* No species. Tachydromia Melgen, 1803, is a change of name. Type, Tachydromia connexa Melgen. Syn., Sicus Latreille, 1796, preoccupied; Tachydromia Meigen, 1803; Tachista Loew, 1860.

$\checkmark$ Corynoneura Winnertz, Ent. Zeit. Stettin, vol. 7, p. 12, 1846. 2 species. Type, Corynoneura scutellata Winnertz, the first species, by present designation.

$\checkmark$ Cotilea Lioy, Atti Inst. Veneto, ser. 3, vol. 9, p. 1123, 1864. 1 species. Type, Chlorops gracilis Meigen. Equals Titania Meigen, 1800.

$\checkmark$ Crameria Desvoldy, Essai Myod.,p. 59, 1830. 1 species. Type, Crameria astroidea Desvoldy. Equals Trixa Meigen, 1824.

$\checkmark$ Crassiseta von Roser, Corr. Landw. Ver. Wurtemburg for 1840, p. $63,1840{ }^{*} 1$ species (as 5). Type, Oscinis cornuta Fallen. Syn., Macrochetum Rondani, 1856.

$\checkmark$ Cricotopus Wulp, Tijdschr. Ent., vol. 17, p. 132, 1874. 9 species. Type, Chironomus tibialis Melgen, the first species, by present designation.

$\checkmark$ Crioprora Osten SAcken, Cat. Dipt. N. Amer., p. 251, 1878. 3 species. Type, Pocota alopex Osten Sacken, the third species, by designation of Williston, Synops. N. Amer. Syrph., 1886, p. 217. Syn., Romaleosyrphus B1Goт, 1882.

$\mathcal{J}$ Criorhina Meigen, Syst. Beschr. Zweifl. Ins., vol. 3, p. 236, 1822. 6 species. Type, Syrphus asilicus Fallen, the fifth species, by designation of Westwood, Intr., vol. 2, Synops., 1840, p. 136 . Equals Penthesilia Meigen, 1800.

$\checkmark$ Crocuta Meigen, Nouv. Class. Mouch., p. 84, 1800.* No species. Siphona Meigen, 1803, is a change of name. Type, Musca geniculata De Geer. Syn., Siphona Me1gen, 1803; Bucentes Latreille, 1809.

$\checkmark$ Crossocosmia Mıк, Wien. Ent. Zeit., vol. 9, p. 313, 1890. 1 species. Type, Ugimyia sericarix Rondanı. Equals Sturmia Desvoldy, 1830.

$\checkmark$ Crossopalpus B1Got, Ann. Soc. Ent. France for 1857 , p. 557, 1857. 1 species. Type, Platypalpus ambiguua Macquart. Equals Drapetis Meigen, 1822.

$\checkmark$ Crunobia Kolenat1, Wien. Ent. Monatschr., vol. 4, p. 391, 1860. 1 species. Type, Crunobia schineri Kolenati. Equals Trycyphona Zetrenstedt, 1837.

$\checkmark$ Cryptaneura Bıяот, Rev. Mag. Zool., vol. 11, p. 307, 1859. 1 species. Type, Chrysogaster nitidus WIEDEMANn (as hieroglyphica, new species). Equals Chrysogaster Meigen, 1800.

Cryptochetum Rondani, Bull. Soc. Ent. Ital. for 1875, p. 172, 1875. 1 species. Type, Cryptochetum grandicorne Rondani. Syn., Lestophonus Williston, 1888.

Cryptodiplosis K1EFFer, Bull. Soc. Ent. France for 1895, p. 194, 1895. 1 species. Type, Tipula pini De Geer. Equals Itonida Meigex, 1800.

Cryptolabis Osten SAcken, Proc. Acad. Nat. Sci. Phila. for 1859, p. 224, 1860.1 species. Type Cryptolabis paradoxa Osten Sacken.

Cryptomeigenia Brauer and Bergenstamm, Denkschr. Kais. Akad. Wiss. Wien, vol. 58, p. 311, 1891. 1 species. Type, Cryptomeigenia setifacies Brauer and Bergenstamm. Syn., Emphanopteryx Townsend, 1892.

$\checkmark$ Cryptonevra Lioy, Atti Inst. Veneto, ser. 3, vol. 9, p. 1125, 1864. 1 species. Type, Cholrops flavitarsis Meigen. Syn., Haplegis Loew, 1866.

Cryptopalpus Rondani, Nuovi Ann. Sci. Nat. (Bologna), ser. 3, vol. 2, p. 169, 1850. 3 species. Type, Micropalpus ornatus Macquart, the first species, by present designation. Equals Epalpus Rondani, 1850. 
Ctenoceria Rondani, Dipt. Ital. Prodr., vol. 1, p. 187, 1856. 1 species. Type, Ptychoptera pectinata Maceuart. Equals Liriope M̂teigen, 1800.

Ctenocnemis Kowarz, Verh. Zool.-Bot. Ges. Wien, vol. 22, p. 460, $1873 . \quad$ Change of name for Sturmia Desvoldy, 1830, previously used in Botany, and Blepharipa Rondanl, 1856, on the score of the faulty construction of the latter. Type, Nemorcea squtellata Desvoidy, Equals Sturmia Desvoidy, 1830.

Ctenophora MeIgen, Illiger's Mag., vol. 2, p. 263, 1803. 4 species. Type, Tipula atrata Linnæus, the third species, by designation of Latreille, Consider. Général., 1810, p. 442. Equals Flabellifera Melgen, 1800. (Ctenophora of authors equals Phoroctenia Coquilletr.)

Culex Linnæus, Syst. Nat., 10th ed., p. 602, 1758. 6 species. Type, Culex pipiens Linn eus, the first species, by designation of Latreille, Consider Général., 1810, p. 442. Syn., Heteronycha Arribalzaga, 1891; Neoculex Dyar, 1905.

$\checkmark$ Culicada Felt, Mosq. Culic: N. Y. State, p. 391b, 1904. 7 species. Type, Culex canadensis TheоваLD, the first species, by original designation. Equals Ochlerotatus Arribalzaga, 1891.

Culicella Feut, Mosq. Culic. N. Y. State, p. 391c, 1904. 1 species. Type, Culex dyari COQUillett.

Culicelsa Felt, Mosq. Culic. N. Y. State, p. 391b, 1904. 2 species. Type, Culex treniorhynchus Wiedemans, the first species, by original designation. Equals Ochlerotatus Arribalzaga, 1891.

Culicoides Latreille, Gen. Crust. et Ins., vol. 4, p. 251, 1809. 1 species. Type, Culex pulicaris Linnaus (as punctata, new species). Syn., Forcipomyia Meigen, 1818; Labidomyiä Stephens, 1829; Ecacta Poey, 1851.

Culiseta Felt, Mosq. Culic. N. Y. State, p. 391c, 1904. 2 species. Type, Culex absobrinus FELr, the first species, by original designation.

Cuphocera Macruart, Ann. Soc. Ent. France for 1845, p. 267, 1845. 1 species. Type, Micropalpus ruficomis Macquart. Syn., Palpibraca Rondani, 1845; Copeerypta Townsend, 1908; Deopalpus Townsend, 1908.

Curtonerra Macquart, Ins. Dipt. Nord France, Ather., p. 146, 1834. 12 species. Type, Musca maculata Scopoli, the ninth species, by designation of Westwoon, Intr., vol. 2. Synops., 1840, p. 141. Equals, Graphomya Desvo1by, 1830.

Curtonotum Macevart, Dipt. Exot., vol. 2, pt. 3, p. 193, 1843. 1 species. Type. Musca gibba Fabricius. Syn., Diplocentra Loew, 1859.

Cuterebra Clark, Essay on Bots, p. 70, 1815.* 4 species. Type, CEstrus cunicule Clark, the first species, by present designation. Syn., Trypoderma Wiedemann, 1830.

Cycloleppteron Theobald, Journ. Trop. Med., vol. 4, p. 234, July 15, 1901 . No species. In his Monogr. Culic., vol. 2, p. 312, Nov. 23, 1901, 1 species. Type, Anopheles grabhamii TнеовацD.

Cyclorhynchus Macquart, Dipt. Exot., vol, 2, pt. 1, p. 114, 1840. 1 species. Type, Cyclorhynchus testaceus MaćQuart. Equals Phthiria Meigen, 1803.

Cylindrogaster Lioy, Atti Inst. Veneto, ser. 3, vol. 9, p. 1329, 1864.1 species. Type, Conops ferrugineu Linn жus. Equals Sicus Scopoli, 1763.

$\checkmark$ Cylindromyia Meigen, Illiger's Mag., vol. 2, p. 279, 1803. 1 species. Type, Musca bassicaria Fabricius. Syn., Ocyptera Latreille, 1805; Parthenia Desvoldy, 1830.

Cylindrotoma Macedart, Hist. Nat., Dipt., vol. 1, p. 107, 1834. 3 species. Type, Limnotria distinctissima MElGen, the first species, by designation of Westwood, Intr., Nol. 2, Synops., 1840, p. 128.

Cyniphes Costa, Ann. Accad. Aspir. Nat., vol. 1, p. 4, 1843.* 1 species. Type, Bibio papatasii Scopoll (as molestus, new species). Equals Flebotomus Rondan, 1840.

Cynipimorpha Brauer, Denkschr. Kais. Akad. Wiss. Wien, vol。49, p. 19, 1882. 1 species. Type, Cynipimorpha bilimeki Brauer.

Proc.N.M.vol.37-10-34 
$\checkmark$ Cynisca Desvoidr, Hist. Nat. Dipt., vol. 1, p. 200, 1863. 1 species. Type, Tachina aricola Meigen. Equals Aplomya Desvoidy, 1830.

$\checkmark$ Cynomya Desvordy, Essai Myod., p. 363, 1830. 4 species. Type, Musca mortuorum Linsaus, the first species, by designation of Macquart, Recueil Soc. Sci. Arts Lille, 1834, p. 174. Syn., Cynophaga Lioy, 1864.

J Cynophaga Lioy, Atti Inst. Veneto., ser. 3, vol. 9, p. 890, 1864. Change of name for Cynomya Desvoidy, 1830, because of Conomyia Latreille, 1796. Type, Musca mortuorum Linneus. Equals Cynomya Desvoidy, 1830.

Cynorhina Williston, Synops. N. Amer. Syrph., p. 209, 1886. 2 species. Type, Milesia analis MACQUart, the first species, by present designation. Equals Penthesila MeiGEN, 1800.

$\checkmark$ Cyphomyia Wiedemann, Zool. Mag., stuck 3, p. 55, 1819. 3 species. Type, Cyphomyia auriflamma Wiedemann, the first species, by designation of Brauer, Denkschr. Kais. Akad. Wiss. Wien, vol. 44, 1882, p. 87.

Cypsela Meigen, Nouv. Class. Mouch., p. 31, 1800* No species. Borborus Meigen, 1803, is a change of name. Type, Musca subsultans Fabricius. Syn.; Borborus Meigen, 1803; Sphrocera Latreille, 1805; Lordatia Desvoidy, 1830; Coprina Desvoidy, 1830; Heteroptera Macquart, 1835; Coproica Rondani, 1861; Lotobia Lioy, 1864 .

Cyrillia Desvordy, Hist. Nat. Dipt., vol. 2, p. 31, 1863. 1 species. Type, Tachina nigripes Fallen (as Blondelia fasciata Desvoidy). Equals Lydella Desvoidr, 1830.

$\checkmark$ Cyrtoma Meigen, Syst. Beschr. Zweifl. Ins., vol. 4, p. 1, 1824. 3 species. Type, Empis spuria FAllex, the first species, by designation of Westwood, Intr., vol. 2, Synops., 1840, p. 133 . Equals Bicellaria Macquart, 1823.

Cyrtometopa Loew, Monogr. Dipt. N. Amer., vol. 3, p. 179, 1873. 1 species. Type, Odontomera ferruginea Macquart. Equals Odontomera Macquart, 1843.

Cyrtoneurina Giglio-Tos, Boll. Mus. Zool. Univ. Torino, vol. 8, no. 147, p. 5, 1893. 8 species. Type, Cyrtoneurina uber Giglio-Tos, the eighth species, by present designation. Syn., Clinopera WulP, 1896.

Cyrtophleba Rondani, Dipt. Ital. Prodr., vol. 1, p. 68, 1856. 1 species. Type, Tachina ruricola MEIGEN.

$\checkmark$ Cyrtopogon LoEw, Linn. Ent.,vol. 2, p. 516, 1847. 4 species. Type, Asilus ruficornis FABRicius, the first specics, by designation of Rondanl, Dipt. Ital. Prodr., vo1. 1, 1856, p. 157. Syn., Euarmostus WALKER, 1851; Eupalamus J ENNICKE, 1867.

$\checkmark$ Cyrtosoma Brauer and Bergenstamm, Denkschr. Kais. Akad. Wiss. Wien, vol. 58, p. 372,1891 . No species. In vol. 60, p. 132, 1893, 1 species. Type, Cyrtosoma rufum Brauer and Bergenstamm. Not Cyrtosoma Walker, 1829.

Dactiliscus Rondani, Dipt. Ital. Prodr., vol. 1, p. 158, 1856. 1 species. Type, Asilus striatus FABRICIUS. Equals Habropogon LoEw, 1847.

Dactylocladius KiefFer, Mem. Soc. Sci. Bruxelles, vol.30, p. 356, 1906. 1 species. Type, Orthocladius brevicornis K1EFFer. Equals Orthocladius Wulp, 1874.

Dactylolabis Osten Sacken, Proc. Acad. Nat. Sci. Phila. for 1859, p. 240, 1860. 1 species. Type, Limnophila montana Osten Sacken. Equals Phylidorea Bigot, 1854. Dæochæta Townsend, Trans. Amer. Ent. Soc., vol.19, p. 97, 1892. 1 species. Type, Drochrta harveyi Townsend.

$\checkmark$ Dalmannia Desvoldy, Essai Myod., p. 248, 1830. 6 species. Type, Myopa punctata

Fabricius, the third species, by designation of Rondani, Dipt. Ital. Prodr., vol. 1, 1856, p. 59. Syn., Stachynia Macedart, 1834; Arpagita Lioy, 1864.

[Damalis Fabricius, Syst. Antliatorum, p. 147, 1805. 4 species. Type, Damalis curvipes Fabricius, the first species, by designation of Westwood, Ann. Soc. Ent. France for 1835, p. 684. Not American (or equals Noza Meigen?).] (Damalis of authors equals Xenomyza Wiedemann.)

$\checkmark$ Dasineura Rondan1, Mem. Sec. Ditt. Ital., p. 18, 1840**2 species. Type, Dasineura luteofusca Rondanl, the first species, by present designation. Syn., Perrisia Rondanl, 1846. 
Dasiopa Rondani, Dipt. Ital. Prodr., vol. 1, p. 120, 1856. 1 species. Type, Chortophila lasiophthalma MAcQuart (as loncheus, new species). Equals Lonchæa FALLEN, $18: 0$.

Dasylechia Willistox, Journ. N. Y. Fnt. Soc., vol. 15, p. 1, 1907. 1 species. Type, Hyperechia atrox WILLISTON.

Dasyllis Loew, Bemerk. Asiliden, p. 20, 1851. 4 species. Type, Laphria hæmorrhoa Wiedemann, the first species, by present designation.

Dasymyia EGGER, Verh. Zool.-Bot. Ges. Wien, vol. 8, p. 711, 1858. 1 species. Type, Musca apiformis Schrank. Equals Pocota St. Fargeau and Serville, 1828.

Dasyna Desvoldy, Essai Myod., p. 667, 1830. 2 species. Type, Dasyna fuscipennis Desvoidy, the first species, by present designation. Equals Chyliza Fallen, 1820.

Dasypogon Meigen, Illiger's Mag., vol. 2, p. 270, 1803. 2 species. Type, Asilus diadema FaBricius, the second species, by designation of LATreille, Consider. Général., 1810, p. 443. Syn., Seilopogon Costa, 1854; Cheilopogon Rondan1, 1856.

Dasyptera ScHiner, Wien. Ent. Monatschr., vol. 7, p. 221, 1863. 1 species. Type, Erioptera varia Meigen. Equals Ormosia Rondani, 1856.

Daulopogon Loew, Berliner Ent. Zeitschr., vol. 18, p. 377, 1874. Change of name of Lasiopogon Loew, 1847, on account of an earlier use of this name for a genus of plants. Type, Dasypogon pilosellus LoEw. Equals Lasiopogon Loew, 1847.

Defilippia Lioy, Atti Inst. Veneto, ser. 3, vol. 9, p. 733, 1864. 2 species. Type, Anthrax minos. MeIgen, the second species, by present designation. Equals Mima Meigen, 1820.

Degeeria Meigen, Syst. Beschr. Zweifl. Ins., vol. 7, p. 249, 1838. 16 species. Type, Tachina collaris FALLEN, the seventh species, by designation of Rondan, Dipt. Ital. Prodr., vol. 1, 1856, p. 72. Equals Medina Desvoidy, 1830.

Deinocerites Theobald, Journ. Trop. Med., vol. 4, p. 235, July 15, 1901. No species. In his Monogr. Culic., vol. 2, p. 215, November 23, 1901, 1 species. Type, Deinocerites cancer Theobald. Syn., Brachiosoma Theobald, July 15, 1901; Brachiomyia Theobald, November 23, 1901.

Dejeania Desvoldy, Essai Myod., p. 33, 1830. 2 species. Type, Stomoxys bombylans FABRICIUS, (as capensis, new species), the second species, by present designation. $\checkmark$ Delia Desvoldy, Essai Myod., p. 571, 1830. 30 species. Type, Anthomyia cardui Meigen (as floricola, new species), the first species, by present designation. Equals Hylemya Desvoidy, 1830.

Delphinia Desvordy, Essai Myod., p. 719, 1830. 1 species. Type, Musca picta FAbricius (as thoracica, new species). Syn., Camptonevra Macquart, 1843.

Demoticus Macquart, Ann. Soc. Ent. France for 1854, p. 442, 1854. 1 species. Type, Tachina plebejus Fallen. Syn., Entomobosca Lioy, 1864; Parafischeria TownSEnd, 1908; Neofischeria Townsend, 1908; Apachemyia Townsend, 1908.

Dendromyia Theobald, Monogr. Culic., vol. 3, p. 313, 1903. 5 species. Type, Dendromyia ulocoma TнеоваLD, the first species, by present designation.

$\checkmark$ Dendrophila Lioy, Atti Inst. Veneto, ser. 3, vol. 9, p. 909, 1864. 1 species. Type, Musca hilaris Fallen. Not Dendrophila Swainson, 1837. Equals Eustalomyia Kowarz, 1873.

Dentifibula Felt, 23d Req. State Ent. N. Y., p. 389, 1908. 3 species. Type, Cecidomyia viburni FeLt, the third species, by original designation.

Deopalpus Townsend, Smiths. Misc. Coll., vol. 51, p. 110, 1908. 1 species. Type, Micropalpus californiensis MaCqUaRT (as hirsutus, new species). Equals Cuphocera MACQUART, 1845.

Deromyia Philippi, Verh. Zool.-Bot. Ges. Wien, vol. 15, p. 705, 1865. 3 species. Type, Deromyia gracilis Prillippi, the first species, by designation of Williston, Biol. Centr.-Amer., Dipt., vol. 1, 1901, p. 311. Syn., Diogmites Loew, 1866.

Desmatomyia Williston, Kansas Univ. Quart., vol. 3, p. 268, 1895. 1 species. Type, Desmatomyia anomala W1Lliston. 
Desmatoneura Williston, Kansas Univ. Quart., vol. 3, p. 267, 1895.1 species. Type, Desmatoneura argentifrons WiLLISTON.

/ Desmometopa Loew, Berliner Ent. Zeitschr., vol. 9, p. 185, 1865. 2 species. Type. Agromyza m-atrum MeIgen, the second species, by designation of Hendel, IVien. Ent. Zeit., vol. 22, 1903, p. 251 . Syn., Macrurus Lioy, 1864, preoceupied.

Dexia Meigen, Syst. Beschr. Zweifl. Ins., vol. 5, p. 33, 1826. 24 species. Type, Musca rustica Fabricius, the twenty-second species, by designation of Westwool, Intr., vol. 2, Synops., 1840, p. 140. Syn., Dexilla Westwood, 1840; Ida Desvordy, 1863.

Dexilla Westwood, Intr., vol. 2, Synops., p. 140, 1840. 1 species. Type, Muscu rustica Fabricius. Equals Dexia MeIgen, 1826.

$\checkmark$ Dexiopsis Pokorny, Verh. Zool.-Bot. Ges. Wien, vol. 43, p. 533, 1893. 1 species. Type, Aricia lacteipennis ZetTerstedt.

$\checkmark$ Dexiosoma Rondani, Dipt. Ital. Prodr., vol. 1, p. 85, 1856. 1 species. Type, Arusca canina FABricius.

Dexodes Brauer and Bergenstam, Denkschr. Kais. Akad. Wiss. Wien, vol. 56, p. 87, 1889. 1 species. Type, Tachina albisquama Zetterstent (as spectubitis Meigen). Equals Lydella Desvoiny, 1830.

Diabasis Macquart, Hist. Nat., Dipt., vol. 1, p. 207, 1834. 4 species. Type, Tubanus bicinctus Fabricius, the first species, by present designation. Not Diabasis Hoffmannsegg, 1819. Equals Diachlorus Osten Sacken, 1876.

$\checkmark$ Diachlorus Osten SAcken, Mem. Boston Soc. Nat. Hist., vol. 2, p. 475, 1876. Change of name for Diabasis Macquart, 1834, not of HoffmannsegG, 1819. Type, Tabanus bicinctus Fabricius. Syn., Diabasis Macquart, 1834, preoccupied.

Diacrita Gerstacker, Ent. Zeit. Stettin, vol. 21, p. 195, 1860. 1 species. Type, Diacrita costalis Gerst.ecker. Syn., Carlottrmyia Bigot, 1877.

$\checkmark$ Diadocidia Ruthe, Isis von Oken for 1831, p. 1210, 1831. 1 species. Type Ifycetooia fermiginosa Meigen (as flavicans, new species). Syn., Macronevra Macquart, 1834.

$\checkmark$ Dialineura Rondani, Dipt. Ital. Prodr., vol. 1, p. 155, 1856. 1 species. Type, Musca anilis Linneus. Equals Thereva Latreille, 1796.

Dialysis Walker, Ins. Saund., Dipt., p. 4, 1850. 1 species. Type, stygica elonguta SAY (as dissimilis, new species). Syn., Agnotomyia Williston, 1886.

Diamesa Meicien, Syst. Beschr. Zweifl. Ins., vol. 7, p. 12, 1838. 2 species. Type, Diamesa raltlii M EIGEN, the first species, by present designation.

$\checkmark$ Ihia horopeza Townsend, Smiths. Misc. Coll., vol.51, p. 66, 1908. 1 species. Type, Atrophopoda braueri Williston. Equals Paradidyma Brauer and Bergenstani, 1891.

J Diaphorus Meigen, Syst. Beschr. Zweifl. Ins., vol. 4, p. 32, 1824. 4 species. Type, Dolichopus oculatus FALLEN (as flavocinctus, new species), the first species, by designation of Westwood, Intr., vol. 2, Synops., 1840, p. 134. Syn., Brachypus MeIgen, 1824; Lyroncurus LoEw, 1857.

Diasemocera Bezzi, Wien. Ent. Zeit., vol. 14, p. 137, 1895. 1 species. Type, Psilopa röderi Girschner (as nigrotrniata, new species). Equals Psilopa Fallen, 1823.

[Diastata Meigen, Syst. Beschr. Zweifl. Ins., vol. 6, p. 94, 1830. 16 species. Type, Geomyza obscurella FALLEN, the third species, by designation of WEstwoon, Intr., vol. 2, Synops., 1840, p. 152. Not American]. (Diustata of authors equals Calopterella, new name.)

Diathronomyia FeLt, 23rl Rep. State. Ent. N. Y., p. 339, 1908. I species. Type, Diathronomyia artemisix FeLT.

Diatomineura Rondani, Arch. Zool., vol. 3, p. 84, 1864. 5 species. Type, Pangonius depressus MacruarT, the first species, by present designation. Equals Pangonius Latreille, 1802.

Dichæta Metgen, Syst. Beschr. Zweifl. Ins, vol. 6, p. 61, 1830. 1 species. Type, Notiphila caudata Fallen.

Dichætoneura JoHnson, Psyche, vol. 14, p, 9, 1907, 1 species. Type, Dichætoneura leucoptera JoHnson. 
Dichelacera Macquart, Dipt. Exot., vol. 1, pt. 1, p. 112, 1838 . 4 species. Type, Dichelacera unifasciata MACQUART, the third species, by present designation.

Dichelomyia Rubsaanen, Berliner Ent. Zeitschr., vol. 37, p. 346, 1892. 21 species. Type, Cecidomyia saliciperda Dufour, the second species, by present designation. Equals Rabdophaga Westwood, 1847.

Dichocera Williston, Ent. News, vol. 6, p. 31, 1895. 1 species. Type, Dichocera lyrata WILLISTON.

Dichrodiplosis Kieffer, Bull. Soc. Ent. France for 1895, p. 194, 1895. 1 species. Type, Dichrodiplosis faciate KiefFer.

Diclisa Schiner, Verh. Zool.-Bot. Ges. Wien, vol. 17, p. 311, 1867. 1 species. Type, Pangonius incompletus Macquart. Equals Scione Walker, 1850.

Dicolonus Loew, Berliner Ent. Zeitschr., vol. 10, p. 32, 1866. 1 species. Type, Dicolonus simplex LoEw.

Dicranomyia Stephens, Syst. Cat. Brit. Ins., vol. 2, p. 243, 1829. 8 species. Type, Limnobia modesta Melgen, the fourth species, by present designation. Equals Furcomyia Meigen, 1818.

Dicranophragma Osten Sacken, Proc. Acad. Nat. Sci. Phila. for 1859, p. 240, 1860. 1 species. Type, Limnophila fuscovaria Osten Sacken. Equals Phylidorea Bigot, 1854.

Dicranoptycha Osten Sacken, Proc. Acad. Nat. Sci. Phila. for 1859, p. 217, 1860. 4 (supposed) species. Type, Dicranoptycha germana Osten SACKen, the first species, by present designation. Equals Marginomyia Meigen, 1818.

Dicranota Zetrenstedt, Ins. Lapp., p. 851, 1838. 1 species. Type, Tipula bimaculata Schumnel (as guerini, new species).

Dicranus Loww, Bemerk. Asiliden, p. 13, 1851. 1 species. Type, Dasypogon rutilus IVIEDEMANN.

Didea Macquart, Hist. Nat., Dipt., vol. 1, p. 508, 1834. 1 species. Type, Didea fasciata Macquart: Syn., Enica Meigen, 1838.

. Didyma Wulp, Biol. Centr.-Amer., Dipt., vol. 2, p. 156, 1890. 18 species. Type, Didyma albomicans Wulp, the twelfth species, by designation of TownsEND in Williston's Man. N. Amer. Dipt., 1908, p. 379.

Dilophus Meigen, Illiger's Mag., vol. 2, p. 264, 1803. No species. In his Klass. Beschr. Zweifl. Ins., 1804, pp. 115 and 116, 3 species. Type, Tipula febrilis Linneus, the first species, by designation of Latreille, Consider. Général., 1810, p. 442. Equals Philia Meigen, 1800.

$\checkmark$ Dimeraspis Newman, Ent. Mag., vol. 5, p. 372, 1838. 1 species. Type, Mulio glołosus Fabricius (as podagra, new species). Equals Microdon Melgen, 1803.

$\checkmark$ Dinerra Liox, Atti Inst. Veneto, ser. 3, vol. 9, p. 1315, 1864. 4 species. Type, Phytomyza elegans Melgen, the firstspecies, by presentdesignation. Equals Napomyza HaLIDAY, 1840.

Dioctria Meigen, Illiger's Mag., vol. 2, p. 270, 1803. 3 species. Type, Asilus celandicus Linnæus, the first species, by designation of Latreille, Consider. Général., p. 443,1810 .

Diogmites Loew, Berliner Ent. Zeitschr., vol. 10, p. 21, 1866. 8 species. Type, Diogmites platypterus LoEw, the first species, by present designation. Equals Deromyia Philippi, 1865.

Diomonus WaLker, List Dipt. Brit. Mus., vol. 1, p. 87, 1848. 1 species. Type, Diomonus nebulosus WaLKER.

$\checkmark$ Diomyza Meigen, Syst. Beschr. Zweifl. Ins., vol. 1, p. 89, 1818. 1 species. Type, Lasioptera picta Meigen. Equals Lasioptera Meigen, 1818.

$\checkmark$ Dionæa Desvoldy, Essai Myod., p. 253, 1830. 2 species. Type, Tachina forcipata Meigen, the first species, by designation of Desvoldy, Hist. Nat. Dipt., vol. 2, 1863, p. 54. Syn., Labigastera Macquart, 1834. 
- Dionnæa Meigen, Nouv. Class. Mouch., p. 24, 1800* No species. Platyptera Meigen, 1803, is a change of name. Type, Empis platyptera Panzer. Syn., Platyptera Meigen, 1803; Macrostomus Wiedemann, 1817; Rhamphomyia Meigen, 1822; Enicopteryx Stephens, 1829; Holoclera Schiner, 1860; Megacyttarus Bigot, 1880.

- Diostracus Loew, Neue Beitr., vol. 8, p. 43, 1861. 1 species. Type, Diostracus prasinus LoEw.

$\checkmark$ Diotrepha Osten Sacken, Cat. Dipt. N. Amer., p. 219, 1878.1 species. Type, Diotrepha mirabilis Osten SACKEN.

Dipalta Osten Sacken, Bull. 3, U. S. Geol. Surv., p. 236, 1877. 1 species. Type, Dipalta serpentina Osten SACKEN.

J Diphysa Macquart, Dipt. Exot., vol. 1, pt. 1, p. 172, 1838. 2 species. Type, Xylophagus rufipalpus WiedemanN, the second species, by present designation. Not Diphysa Blainville, 1834. Equals Exaireta Schiner, 1867.

$\checkmark$ Diplocentra Loew, Zeitschr. Ent. Breslau, vol. 13, p. 13, 1859. Change of name for Curtonotum Macquart, 1843, on account of alleged preoccupation. Type, Musca gibba Fabricius. Equals Curtonotum MacQUart, 1843.

Diplomera Lioy, Atti Inst. Veneto, ser. 3, vol. 9, p. 1349, 1864. 2 species (as 3).

Type, Tachina bisignata MElgen, the first species (as biguttata MElGeN), by present designation. Equals Meigenia Desvordy, 1830.

Diplosis Loew, Dipt. Beitr., vol. 4, p. 20, 1851. 15 species. Type, Tipula pini DE Geer, the ninth species, by designation of Rondani, Atti Soc. Ital. Sei. Nat., rol. 2, 1861, p. 289. Equals Itonida MEIGEN, 1800.

$\checkmark$ Diplotoxa Loew, Berliner Ent. Zeitschr., vol. 7, p. 54, 1863. 1 species. Type, Chlorops versicolor LOEw.

$\checkmark$ Dipsa Fallex, Spec. Ent. Meth. Exh., p. 20, 1810, 1 species. Type, Lonchoptera lutea Panzer (as bifurcata, new species). Equals Lonchoptera MeIriex, 1803.

Dirhiza Loew, Dipt. Beitr., vol. 4, p. 21, 1850. 1 species. Type, Dirhiza lateritia LEOW.

$\checkmark$ Discobola Osten Sacken, Proc. Acad. Nat. Sci. Phila. for 1861, p. 226, 1862.2 species. Type, Limnobia argus $\mathrm{S}_{\mathrm{AY}}$, the second species, by present designation. Syn., Trochobola Osten SAcken, 1869.

$\checkmark$ Discocephala Macquart, Dipt. Exot., vol. 1, pt. 2, p. 50, 1838. 3 species. Type, Dasypogon abdominalis $\mathrm{S}_{\mathrm{AY}}$ (as rufiventris, new species), the third species, by present designation. Not Discocephala LAPORTE, 1832. Equals Holcocephala J ENNICKE, 1867.

Discocerina Macquart, Hist. Nat., Dipt., vol. 2, p. 527, 1835. 4 species. Type, Notiphila obscurella Fallen, the first species, by present designation. Syn., Clasiopa STEnhanmer, 1844.

Discomyza Meigen, Syst. Beschr. Zweifl. Ins., vol. 6, p. 76, 1830. 1 species. Type, Psilopa incurva FAllen.

Distichona Wulp, Biol. Centr.-Amer., Dipt., vol. 2, p. 44, 1890. 1 species. Type, Distichona varia Wulp. Syn., Pseudogermaria Brater and Bergexstama, 1891; Olenochata Townsend, 1892.

Ditomyia Winnertz, Ent. Zeit. Stettin, vol. 7, p. 14, 18t6. 1 species. Type, Mycetobia fasciata MEIGEN (as trifasciata, new species).

$\checkmark \quad$ Dixa MeIgen, Syst. Beschr. Zweifl. Ins., vol. 1, p. 316, 1818. 3 species (as 4). Type, Dixa maculata Meigen, the third (valid) species, by designation of Curtis, Brit. Ent., 1832, p. 409.

Dizonias Loew, Berliner Ent. Zeitschr., vol. 10, p. 30, 1866.1 species. Type, Dizonias phcenicurus LoEw.

Docosia Winnertz, Verh. Zool.-Bot. Ges. Wien, vol, 13, p. 802, 1863. 2 species. Type, Docosia valida Winnertz, the second species, by designation of Johannsen, Gen. Ins., Dipt., Mycet., 1909, p. 92. 
Dolichoi:ephala MacquaRt, Monogr. Empides, p. 147, 1823. 1 species. Type, Tachydromia irrorata Fallen (as maculata, new species). Syn., Ardoptera Macquart, 1827; Leptosceles Haliday, 1833.

Dolichocodia Townsend, Smiths. Misc. Coll., vol. 51, p. 59, 1908. 1 species. Type, Myocera bivittata CoquilletT.

Dolichogaster Macquart, Dipt. Exot., Suppl. 3, p. 178, 1848. 1 species. Type, Aydas brevicornis WiEDEMANN.

Dolichoglossa Stern, Berliner Ent. Zeitschr., vol. 42, p. 230, 1898. 1 species. Type, Proboscimyia siphonina Bigot (as americana, new species). Equals Proboscimyia Bigot, 1883.

Dolichomyia Wiedemann, Auss. Zweifl. Ins., vol. 2, p. 642, 1830. 1 species. Type, Dolichomyia nigra Wiedemann.

Dolichopeza Curtis, Brit. Ent., p. 62, 1825. 1 species. Type, Tipula albipes Strom (as silvicola, new species). Syn., Leptina Meigen, 1830; Apeilesis Macquart, 1846.

Dolichopus Latreille, Precis, p. 159, 1796. No species. In his Hist. Nat. Crust. et Ins., vol. 3, 1802, p. 440, 2 species. Type, Musca ungulata Linndes, the first species, by designation of Latreille, Consider. Général., 1810, p. 443 . Syn., Iphia Meigen, 1800; Satyra Meigen, 1803; Ragheneura Rondan1, 1856; Hygroceleuthus LoEw, 1857; Spathichira Bigot, 1888.

Doliosyrphus Bigot, Bull. Soc. Ent. France for 1882, p. 120, 1882. 3 species. Type, Doliosyrphus scutellatus Bigot, the first species, by designation of Williston, Synops. N. Amer. Syrph., 1886, p. 178.

Dorbinia Desvoldy, Ann. Soc. Ent. France for 1847, p. 272, 1847. 2 species (as 4). Type, Musca quadripustulata FABricius (as ludibunda and nitida, new species), the first two supposed species, by designation of Desvordy, Hist. Nat. Dipt., vol. 1, 1863, p. 217. Equals Winthemia Desvordy, 1830.

$\checkmark$ Doria Meigen, Syst. Beschr. Zweifl. Ins., vol. 7, p. 263, 1838. 4 species. Type, Tachir a concinnata Meigen, the first species, by designation of Desvoidy, Hist. Nat. Dipt., vol. 1, 1863, p. 535. Equals Compsilura Bouche, 1834.

Dorilas Meigen, Nouv. Class. Mouch., p. 31, 1800.* No species. Microcera Meigen, 1803, is a change of name. Type, Pipunculus campestris Latreille. Syn., Pipunculus Latreille, 1802; Microcera Meigen, 1803; Cephalops Fallen, 1810; Prothecus Rondani, 1856; Alloneura Rondani, 1856.

Doryclus J JnNicka, Abh. Seckenb. Ges., vol. 6, p. 365, 1867. 1 species. Type, Asilus distendens Wiedemann. Equals Ampyx Walker, 1855.

Drapetis Meigen, Syst. Beschr. Zweifl. Ins., vol. 3, p. 91, 1822. 1 species. Type, Drapetis exilis Meigen. Syn., Crossopalpus Bigot, 1857.

Drepanoglossa Townsend, Trans. Amer. Ent. Soc., vol. 18, p. 377, 1891. 1 species. Type, Drepanoglossa lucens Townsend. Equals Epigrimyia Townsend, 1891.

Drepanomyia Wheeler, Zool. Bull., vol. 1, p. 217, 1898. 2 species. Type, Drepanomyia pruinosa WHEELER, the first species, by present designation. Equals Hypocharassus Miк, 1879.

Drepanophora Strobl, Programm Seiten., vol. 14, p. 40, 1880* 1 species. Type, Phora galeata Haliday (as braueri, new species). Equals Metopina Macquart, 1835.

Drosophila Fallen, Geomyzides Sveciæ, p. 4, 1823.* 12 species. Type, Musca funebris Fabricius, the third species, by designation of Curtis, Brit. Ent., 1833, p. 473 (as cellaris Meigen, not Linneus).

Drymeia Meigen, Syst. Beschr. Zweifl. Ins., vol. 5, p. 204, 1826. 1 species. Type, Musca hamata FALLEN (as obscura, new species).

Dryomyza Fallen, Sciomyz. Sveciæ, p. 15, 1820. 2 species. Type, Musca flaveola Fabricius (as vetula, new species), the first species, by designation of Westwood, Intr., vol. 2, Synops., 1840, p. 145. Syn., Dryope Desvoldr, 1830. 
$J$ Dryope Desvoidy, Essai Myod., p. 618, 1830. 2 species. Type, Musca flareola Fabricius (as communis, new species), the first species, by present designation. Equals Dryomyza Fallen, 1820.

Duponchefic Desroidy, Hist. Nat. Dipt., vol. 1, p. 531, 1863. 1 species (as 2).

Type, Chetogena segregata Rondan1 (as sylwestris and infuscata, new species). Not Duponchelia Zeller, 1847. Equals Parasetigena Brauer and Bergenstama, 1891.

Dynatosoma Winnertz, Verh. Zool.-Bot. Ges. Wien, vol. 13, p. 947, 1863. 2 species. Type, Mycetophila fuscicornis Meigen, the first species, by designation of Johandsex, Gen. Ins., Dipt., Mycet., p. 114, 1909.

$\gamma$ Dysmachus Lokw, Dipt.-Fauna Südafrika, p. 143, 1860. 4 species. Type, . Asitus trigomus MElgen, the third species, by present designation. Syn., Lophonotus MACQUART, 1838, preoceupied.

$\checkmark$ Earomyia Zetrenstedt, Dipt. Scand., vol. 1, p. 78, 1842. No species. In vol. 7, 1848, p. 2690, 1 species. Type, Earomyia lonchroides Zetrenstent. Equals Lonchæa FALLEN, 1820.

$\checkmark$ Eccoptomera Loew, Zeitschr. Ent. Breslau, vol. 13, p. 47, 1862. 7 species. Type, Ileleomyzu longiseta MeIgen, the second species, by present designation.

$\checkmark$ Eccritosia Schiner, Verh. Zool.-Bot. Ges. Wien, vol. 16, p. 674, 1866. 2 species. Type, Asitus barbatus $\mathrm{F}_{\mathrm{ABR}} \mathrm{cius}$, the first species, by original designation.

$\checkmark$ Eeculex Felt, Mosq. Culic. N. Y. State, p. 391c, 1904. 2 species. Type, Culex syliestris TheовадD, the first species, by original designation. Equals Ochlerotatus A Rribalzaga, 1891.

$\checkmark$ Echinodexia Brauer and Bergenstam, Denkschr. Kais. Akad. Wiss. Wien, vol. 60 , 1. 174, 1893. 1 species. Type, Hystrisyphona pseudohystricia Brater and BergenST.M.M.

Echinogaster Lior, Atti Inst. Veneto, ser. 3, vol. 9, p. 1335, 1864. 1 species. Type, Tachina promptu Meigen (as Echinomya argentifrons Macedart). Equals Peleteria Desvoidy, 1830.

$\checkmark$ Echinomya Latreilue, Hist. Nat. Crust. et Ins., vol. 14, p. 377, 1805. 2 species. Type, Ifusca grossa Linnsus, the first species, by designation of Westwood, Intr., yol. 2, Synops., 1840, p. 138. Equals Larvævora Meigen, 1800.

Echthodopa Loww, Berliner Ent. Zeitschr., vol. 10, p. 16, 1866. 1 species. Type, Echthodopa pulera Loew.

$\mathcal{J}$ Ecitomyia Brues, Amer. Nat., vol 35, p. 347, 1901. 1 species. Type, Ecitomyia y.hecleri Brues.

Eclimus Loww, Ent. Zeit. Stettin, vol. 5, p. 15t, 184t. 2 species. Type, Eclimus perspicilluris Loew, the first species, by present designation. Syn., Thevenemyia Bigot, 1875; Epibutes Osten Sacken, 1877.

Ectecephala Macevart, Dipt. Exot., Suppl. 4, pt. 2, p. 280, 1851. 1 species. Type, Ectecephala albistylum MAcruart.

Ectyphus Gerst.ecker, Ent. Zeit. Stettin, vol. 29, 1. 92, 1868. 1 species. Type, Fiftyphus pinguis Gerst.ECK ER.

Efferir Coqulluter, Can. Ent., vol. 25, p. 175, 1893. 5 species. Type, Efferia candirk Coquillett, the fifth species, by present designation. Equals Eichoichemus Bigot, 1857.

Egeria Desvondy, Essai Myod., p. 555, 1830. 3 species. Type, Egeriu siluatica Desvoldy, the first species, by present designation. Equals Hylemya Desroidr; 1830.

$\mathcal{J}$ Eggeria Schiner, Wien. Ent. Monatschr., vol. 5, p. 142, 1861. 1 species. Tyṕe, Fallenu fasciatı EgGer. Equals Phorocera Desvoidy, 1830.

$\checkmark$ Egle Desvoluy, Fssai Myod., p. 584, 1830. 21 species. Type, Egle partu Desvoldy, the twentieth species, by present designation. Equals Pegomya Desvoidy, 1830. 
Eicherax Bigot, Ann. Soc. Ent. France for 1857, p. 545, 1857. 1 species. Type, Erax simplex MACQUART. Syn., Eristicus Loew, 1848, preoccupied; Neoeristicus Osten SACKen, 1878.

Eichoichemus Bigot, Ann. Soc. Ent. France for 1857, p. 543, 1857. 1 species. Type, Erax flavianalis Miacquart. Syn., Efferia Coqúillett, 1893.

[Elachiptera Macquart, Hist. Nat., Dipt., vol. 2, p. 621, 1835.1 species. Type, Chlorops brevipennis Meigen. Not American.]

Elachisoma Rondani, Bull. Soc. Ent. Ital., vol. 12, p. 5, 1880. 1 species. Type, Borborus nigerrimus Haliday. Equals Leptocera Olivier, 1813.

$\mathcal{J}$ Elixotoma Costa, Atti Accad. Sci. Fis. Mat., vol. 1, p. 49, 1863. 1 species. Type, Laphria albibarbis MeIGen (as adustiventris, new species). Equals Andrenosoma RONDAN1, 1856.

$\checkmark$ Elaphropeza Macquart, Ins. Dipt. Nord France, Separata, p. 86, 1827. 1 species. Type, Tachydromia ephippiata Fallen.

$\checkmark$ Elephantomyia Osten SAcken, Proc Acad. Nat. Sci. Phila. for 1859, p. 220, 1860. 1 species. Type, Elephantomyia westwoodi Osten SAcken (as Limnobiorhynchus canadensis WesTWOOD).

$\checkmark$ Elfia Desvordy, Ann. Soc. Ent. France for 1850, p. 190, 1850. 2 species. Type, Actia cingulata Desvordy, the first species, by designation of Desvordy, Hist. Nat. Dipt., vol. 1, 1863, p. 672. Equals Actia Desvoidy, 1830.

$\checkmark$ Elgiva Meiges, Syst. Beschr. Zweifl. Ins., vol. 7, p. 365, 1838. 4 species. Type, DIusca cucullaria Linnæus, the fourth species, by designation of Rondani, Dipt. Ital. Prodr., vol. 1, 1856, p. 106. Syn., Gymnostyla Liox, 1864.

Eliozeta Rondani, Dipt. Ital. Prodr., vol. 1, p. 82, 1856. 1 species. Type, Tachina pellucens Fallen. Syn., Clytia Desvomy, 1830, preoceupied; Clytiomyia Rondani, 1861; Chryseria Desvoidy, 1863; Arisbxa Desvoidy, 1863; Phanigaster Lioy, 1864; Clistomorpha Townsend, 1892; Subclytia Pandelle, 1894; Euclytia Townsend, 1908.

Elliponeura LoEw, Berliner Ent. Zeitschr., vol. 13, p. 44, 1869. 1 species. Type, Elliponeura debilis Loew.

f Elliptera Schiner, Wien. Ent. Monatschr., vol. 7, p. 222, 1863. 1 species. Type, Elliptera omissa SCHINer.

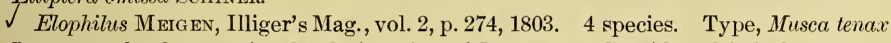
Linn eus, the first species, by designation of Latreille, Consider. Général., 1810, p. 443. Equals Tubifera Meigen, 1800.

Elpe Desvordy, Hist. Nat. Dipt., vol. 1, p. 488, 1863.1 species. Type, Tachina inepta Meigen. Equals Zenilla Drsvoidy, 1830.

$\checkmark$ Elpigia Desvoldy, Hist. Nat. Dipt., vol. 2, p. 149, 1863.1 species (as 4). Type, Miltogramma heteroneura Meigen. Equals Taxigramma Perris, 1852.

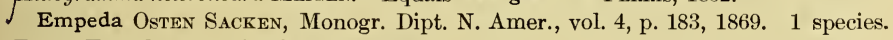
Type, Empeda stigmatica Osten Sacken.

Emphanopteryx Townsend, Trans. Amer. Ent. Soc., vol. 19, p. 120, 1892.1 species. Type, Tachina theutis WALKER (as eumyothyroides, new species). Equals Cryptomeigenia Brauer and Bergenstamm, 1891.

Empheria Winnertz, Verh. Zool.-Bot. Ges. Wien, vol. 13, p. 738, 1863.7 species. Type, Sciophila striata MeIGen, the first species, by present designation. Yot Empheria Hagen, 1856. Equals Neoempheria Osten SACKen, 1878.

Empimorpha Coquilletr, Proc. U. S. Nat. Mus., vol. 18, p. 396, 1896. 2 species. Type, Empimorpha comantis CoquilletT, by original designation.

Empis Linnæus, Syst. Nat., 10th ed., p. 603, 1758. 3 species. Type, Empis pennipes Linnwus, the second species, by designation of Latreille, Consider. Général., 1810, p. 443. Syn. Platypterygia Stephens, 1829; Pachymeria Stephens, 1829; Pachymperina Macquart, 1834; Eriogaster MacquaRT, 1838; Enoplempis Bigot, 1880.

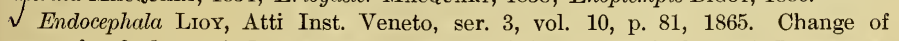
name for Cephenemyia Latreille, 1818, because it is too near Cephalemyia Latreille, 1818. Type, Estrus trompe Linnæus. Equals Cephenemyia Latreille, 1818. 
$\checkmark$ Enica Meigen, Syst. Beschr. Zweifl. Ins, vol. 7, p. 140, 1838. 1 species. Type, Didea fasciata Macquart (as forsteri, new species). Equals Didea Macquart, 1834.

Enicita IVestwood, Intr., vol. 2, Synops., p. 148, 1840. Change of name for Enicopus W WLKer, 1833, not of Stephens, 1830. Type, Sepsis annulipes Meigen. Syn., Enicopus IV'T ALER, 1833, preoceupied.

Enicopteryx Stephens, Syst. Cat. Brit. Ins., p. 264, 1829. 2 species. Type, Rhamphomyia infuscata MEIGEN, the first species, by designation of WESTWOOD, Intr., vol. 2, Synops., 1840, p. 131. Equals Dionnæa Meigen, 1800.

Enicopus WALKER, Ent. Mag., vol. 1, p. 253, 1833. 1 species. Type, Sepsis annulipes Meigen. Not Enicopus Stephens, 1830. Equals Enicita Westwood, 1840.

$\checkmark \quad$ Ennyomma Townsend, Trans. Amer. Ent. Soc., vol. 18, p. 371, Dec., 1891. 1 species. Type, Tachina ænea Wiedemann (as clistoides, new species). Equals Myiophasia Brauer and Bergenstamm, 1891.

Enoplempis Bigot, Bull. Soc. Ent. France for 1880 , p. 47, 1880.1 species. Type, Enoplempis mira Bigot. Equals Empis Linneus, 1758.

$\checkmark$ Ensina Desvoidy, Essai Myod., p. 751, 1830. 1 species (as 6). Type, Musca sonchi Linneus.

$\checkmark \quad$ Enteromyza Rondani, Dipt. Ital. Prodr., vol. 2, p. 20, 1857. Change of name for Gasterophilus LeAch, 1817, and Gastrus Meigen, 1824, because of their inappropriateness. Type, Estrus intestinalis De GeER. Equals Gasterophilus Leach, 1817.

$\checkmark$ Enthenis Desvoidr, Hist. Nat. Dipt., vol. 1, p. 199, 1863. 1 species. Type, Tachina dubia Fallen (as ciligera, new species). Equals Lypha Desvoidy, 1830.

$\checkmark$ Entomobosca Lioy, Atti Inst. Veneto, ser. 3, vol. 9, p. 1350, 1864. 1 species. Type, Tachina plebejus Fallen (as spectabilis Meigen). Equals Demoticus MaCQUART, 1854.

Entomophaga Lioy, Atti Inst. Veneto, ser. 3, vol. 9, p. 1332, 1864.2 species. Type, Tachina exoleta MeIisen, the first species, by present designation. Equals Ceranthia Desvordy, 1830.

$\checkmark \quad$ Epacmus Osten Sacken, Biol. Centr.-Amer., Dipt., vol. 1, p. 142, 1887. Change of name for Leptochilus LoEw, 1872, not of SAussure, 1852. Type, Leptochilus modestus LoEw. Syn., Leptochilus LoEw, 1872, preoccupied.

$\checkmark$ Epalpus Rondani, Nuovi Ann. Sei. Nat. (Bologna), ser. 3, vol. 2, p. 169, 1850. 6 species. Type, Micropalpus rufipennis Macquart, the fourth species, by present designation. Syn., Cryptopalpus Rondani, 1850; Saundersia Schiner, 1868; Rhachoepalpus TownsEND, 1908.

Ephydra Fallen, Spec. Ent. Meth. Exh., p. 22, 1810. No species. In 1813, 4 species. Type, Ephydra riparia FALLen, the first species, by designation of CurTis, Brit. Ent., 1832, p. 413.

$\checkmark$ Ephydrosoma Lioy, Atti Inst. Veneto, ser. 3, vol. 9, p. 1103, 1864. 1 species., Type, Ephydra rufipes Meigen. Equals Napæa Desvoidr, 1830.

$\checkmark$ Ephygrobia Schiner, IVien. Ent. Monatschr., vol. 6, p. 432, 1862. Change of name for Psilopa Fallen, 1823, on account of Psilopus Meigen, 1824. Type, Notiphila nitidula Fallen. Equals Psilopa Fallen, 1823.

Epibates Osten Sacken, Bull. 3, U.S. Geol.Surv., p. 268, 1877. 7 species. Type, Epibates funestus Osten SACKen, the first species, by present designation. Equals Eclimus LoEw, 1844.

Epicypta Winnertz, Verh. Zool.-Bot. Ges. Wien, vol. 13, p. 909, 1863. 3 species. Type, Mycetophila scatophora Perris, the second species, bydesignation of JoHAnnsen Gen. Ins., Dipt., Mycet., 1909, p. 110.

Epidapus HaLiday, in Walker's Ins. Britt., Dipt., vol. 3, p. 56, 1856.1 species. Type, Chionea venatica Haliday. Syn., Atomaria Bigot, 1854, preoceupied.

$\checkmark$ Epidesmia Rondani, Dipt. Ital. Prodr., vol. 1, p. 112, 1856. 1 species. Type, Tephritis cognate Wiedemans. Equals Acidia Desvoidy, 1830. 
Epidiplosis Felt, 23d Rep. State Ent. N. Y., p. 406, 1908. 1 species. Type Epidiplosis sayi FeLT.

Epigrimyia Townsend, Trans. Amer. Ent. Soc., vol. 18, p. 375, 1891.1 species. Type, Epigrimyia polita Townsend. Syn., Drepanoglossa Townsend, 1891; Siphoclytia Townsend, 1892; Siphophyto Townsend, 1892; Coronimyia Townsend, 1892.

Epipela Stenhammar, Kongl. Vet. Akad. Handl. for 1843, p. 143, 1844. 1 species. Type, Ephydra spilota Curtis (as notata, new species). Equals Ilythea HaLIDAY, 1839.

Epiphragma Osten Sacken, Proc. Acad. Nat. Sci. Phila. for 1859, p. 238, 1860. 2 species. Type, Limnobia fascipennis $\mathrm{S}_{\mathrm{AY}}$ (as pavonina, new species), the second species, by present designation.

$\checkmark$ Epiplatea Loew, Berliner Ent. Zeitschr., vol. 11, p. 324, 1868. 1 species. Type, Epiplatea erosa Loew.

$\checkmark$ Epistrophe Walker, Ins. Saund., Dipt., p. 242, 1852. 1 species. Type Musca formosa HARRIs (as conjungens, new species).

Epitriptus Loew, Linn. Ent., vol 4, p. 108, 1849. 6 species. Type, Asilus cingulatus $\mathrm{F}_{\mathrm{ABRICIU}}$, the first species, by present designation.

$\checkmark$ Epochra Loew, Monogr. Dipt. N. Amer., vol. 3, p. 238, 1873. 1 species. Type, Trypeta canadensis LoEw.

$\checkmark$ [Erax Scopoli, Ent. Carn., p. 359, 1763. 14 species. Type, Erax barbatus ScoPoLI, the fifth species, by present designation. Syn., Protophanes Loew, 1860. Not American.] (Erax of authors equals Eicherax Bigot.)

$\checkmark$ Eremomyia Stein, Berliner Ent. Zeitschr., vol. 42, p.223, 1898. 4 species, Type, Eremomyia humeralis Stein, the first species, by designation of Coquillett, Journ. N. Y. Ent. Soc., vol. 9, 1901, p. 137.

Erephopsis Rondani, Arch. Zool., vol. 3, p. 85, 1864. 7 species. Type, Pangonius fulvithorax WrEDEMANN, the second species, by present designation. Equals Pangonius Latreille, 1802.

Eretmoptera Kelloga, Biol. Bull., vol. 1, p. 82, 1900. 1 species. Type, Eretomoptera browni KeLlogG.

Eribea Desvordy, Hist. Nat. Dipt., vol. 1, p. 1033, 1863. 1 species (as 16). Type, Tachina simulans Meigen. Equals Exorista Meigen, 1803.

Erichsonia Desvordy, Hist. Nat. Dipt., vol. 2, p. 481, 1863. 2 species (as35). Type, Sarcophaga hæmorrhoa MeIGEN (the first 24 supposed species), by original designation. Not Erichsmia Westwood, 1849; nor of DANA, 1849. Equals Hartigia DesVOIDY, 1863.

Erigone Desvordy, Essai Myod., p. 65, 1830. 3 species (as 8). Type, Musca radicum FABRICIUS (as anthophila, flavipennis, and scutellaris, new species), the first species, by designation of Desvordy, Hist. Nat. Dipt., vol. 1, 1863, p. 152. Equals Ernestia DesvoIDY, 1830.

Erinia Desvordy, Hist. Nat. Dipt., vol. 1, p. 467, 1863. 1 species. Type, Tachina vulgaris Fallen (as silvatica, new species). Equals Phryxe Desvoldy, 1830.

Erinna Meigen, Nouv. Class. Mouch., p. 21, 1800** No species. Xylophagus MeIGEN, 1803, is a change of name. Type, Nemotelus cinctus De GEer. Syn., Xylophagus Meigen, 1803; Pachystomus Latreille, 1809.

Eriocera Macquart, Dipt. Exot., vol. 1, pt. 1, p. 74, 1838. 1 species. Type, Limnobia nigra Wiedemann. Equals Caloptera Guerin, 1829.

Eriogaster Macquart, Dipt. Exot., vol. 1, pt. 2, p. 162, 1838. 1 species. Type, Empis laniventris Eschscholz. Not Eriogaster Germar, 1811. Equals Empis LinAxwus, 1758.

Erioischia Lioy, Atti Inst. Veneto, ser. 3, vol. 9, p. 991, 1864. 1 species. Type, Chortophila floccosa Macquart. Equals Pegomya Desvoidy, 1830.

Eriophora Philippi, Verh. Zool.-Bot. Ges. Wien, vol. 15, p. 735, 1865. 1 species. Type, Eriophora aureorufa PhilipPi. Equals Penthesilia Meigen, 1800. 
$\checkmark$ Erioptera Meigen, Illiger's Mag., vol. 2, p. 262, 1803. No species. In 1804, 6 species. Type, Erioptera lutea Meigen, the 5th species, by present designation. Equals Polymeda Meigen, 1800.

$\checkmark$. Eriostyla Lioy, Atti Inst. Veneto, ser. 3, vol. 9, p. 997, 1864. 2 species. Type, Coenosia dubia Macquart, the second species, by designation of Coquillett, Journ. N. Y. Ent. Soc., vol. 9, 1901, p. 137. Equals(?) Coenosia Meigen, 1826.

$\checkmark$ Eristalinus Rondani, Nuovi Ann. Sci. Nat. (Bologna) for 1844, p. 453, 1844. No species. In his Dipt. Ital. Prodr., vol. 2, 1857, p. 38, 1 species. Type, Mfusca sepulchralis Linn.eus. Equals Tubifera Meigen, 1800.

$\checkmark$ Eristalis Latreille, Nouv. Dict. Hist. Nat.,vol. 24, p. 194, 1804.* 7 species. Type, Musca tenax Linneus, the third species, by designation of Curtis, Brit. Ent., 1832, p. 432. Equals Tubifera Meigen, 1800.

Eristaloides Rondani, Nuovi Ann. Sci. Nat. (Bologna) for 1844, p. 453, 1844. No species. Eristalomya Rondani, 1857, is a change of name. Type, Musca tenax LinNæus. Equals Tubifera Meigeñ, 1800.

$\checkmark$ Eristalomya Rondani, Dipt. Ital. Prodr.,vol. 2, p. 38, 1857. 1 species. Type, Musca tenax Linneus. Equals Tubifera Meigen, 1800.

$\checkmark$ Eristicus Loew, Linn. Ent., vol. 3, p. 396, 1848. No species. In Abh. Nat. Ver. Sach. Thur. Halle, vol. 2, 1860, p. 213, he stated that the species of this genus were found in America. Osten Sacken, Cat. Descr. Dipt. N. Amer., 1878, p. 79, said that there is a specimen of Erax ambiguus M ACQUART (equals Asilus interruptus MACQUART) in the Museum of Comparative Zoology in Cambridge, Mass., evidently so named by Loew, and as it agrees with the characters of Eristicus, it may therefore be taken as the type. Type, Asilus interruptus Macquart. Not Eristicus Wesmael, 1844. Equals Eicherax Bigot, 1857.

$\checkmark$ Ernestia Desvoldy, Essai Myod., p. 60, 1830. 1 species. Type, Tachina rudis Fallen (as microcera, new species). Syn., Fausta Desvoldy, 1830; Mericia Desvoidy, 1830; Erigone Desvoiny, 1830; Panzeria Denvoidy, 1830; Varichrta Speiser, 1903.

$\checkmark$ Ervia Desvordy, Essai Myod., p. 225, 1830. 1 species. Type, Ocyptera triquetra Olivier. Syn., Paranaphora Townsend, 1908.

$\checkmark$ Erythrandra Brauer and Bergenstamu, Denkschr. Kais. Akad. Wiss. Wien, vol. 58, p. 368, 1891 . 1 species. Type, Erythrandra picipes Brauer and Bergenstamm.

$\checkmark$ Esila Desvoiny, Hist. Nat. Dipt., vol. 1, p. 1063, 1863. 1 species. Type, Tachina simulans Meigen (as arvorim, new species). Equals Exorista Meigen, 1803.

WEstelia Desvomy, Essai Myod., p. 635, 1830. 3 species. Type, Ochtiphila juncorum FALlen (as herbarum, new species), the second species, by present designation. Equals Chamæmyia Mergen, 1803.

Ethilla Desvoidy, Hist. Nat. Dipt., vol. 1, p. 202, 1863. 1 species. Type, Tachina zemulu Meigen. Equals Aplomya Desvoidy, 1830.

Euacaulona Townsend, Smiths. Misc. Coll., vol. 51, p. 131, 1908. 1 species. Type, Eucculone semichrasti Townsend. Equals Acaulona WulP, 1888.

Euantha Wulp, Tijdschr. Ent., vol. 28, p. 198, 1885. 1 species. Type, Ocyptera litturata Olivier (as Dexia dives Wiedemann).

Euaresta Loww, Monogr. Dipt. N. Amer., vol. 3, p. 296, 1873. 10 species. Type, Trypeta festiva Loew, the second species, by present designation.

Euarmostus Walker, Ius. Saund., Dipt., p. 102, 1851. 1 species. Type, Euarmostus bimacula WALKER. Equals Cyrtopogon Loew, 1847.

Eucalliphora Townsend, Smiths. Misc. Coll., vol. 51, p. 118, 1908.1 species. Type, Calliphora latifrons Hovgir. Equals Calliphora DesvoIdy, 1830.

Eucalodexia Townsend, Smiths. Misc. Coll., vol.51, p. 64, 1908. 1 species. Type, Homodexia flavipes Bı̈T.

$\checkmark \quad$ Euceratomyia Williston, Wien. Ent. Zeit., vol. 3, p. 185, 1884. 1 species. Type`' Euceratomyia pergandei Williston. Equals Pelecocera Meigen, 1822. 
Eucessia Coquillett, Can. Ent., vol. 18, p. 82, 1886.1 species. Type, Eucessia mubens COQUilietT.

Euchætogyne Townsend, Smiths. Misc. Coll., vol. 51, p. 59, 1908. 1 species. Type, Hystrichodexia röderi WiLliston.

Euclytia Townsend, Smiths. Misc. Coll., vol. 51, p. 60, 1908. 1 species. Type, Clytia flava Townsend. Equals Eliozeta Rondani, 1856.

Eucnephalia Townsend, Can. Ent., vol. 24, p. 166, 1892. 1 species. Type, Eucnephalia gonordes Townsend.

Eucorethra UNDERWood, Science, vol. 18, p. 182, Aug. 7, 1903.1 species. Type, Eucorethra underwoodi Underwood. Syn., Pelorempis Johannsen, August 11, 1903.

Eudexia Brauer and Bergenstamm, Denkschr. Kais. Akad. Wiss. Wien, vol. 56, p. 120, 1889. 1 species. Type, Rhamphinina formidabilis Bıяот (as goliath, new species).

Eudicrana LoEw, Berliner Ent. Zeitschr., vol. 13, p. 142, 1869. 1 species. Type, Eudicrana obumbrata LoEw.

Eudiplosis Kieffer, Bull. Soc. Ent. France for 1894, p. 28, 1894. 2 MS. species (described in 1896). Type, Contarinia sorbi KIEFFEr, the first species, by present clesignation. Equals Contarinia Rondanl, 1861.

Eudora Desvoiny, Hist. Nat. Dipt., vol. 1, p. 623, 1863. 1 species. Type, Echinomya lefeburei Desivoidy (as illustris, new species). Not Eudora Lesson, 1809. Equals Cnephaotachina Brauer and Bergenstama, 1894.

Eudoromyia Bezzi, Zeitschr. IIym. Dipt., vol. 6, p. 49, 1906. Change of name for Eudora Desvoidy, 1863, not Lesson, 1809. Type, Echinomya lefeburei Desvoiny. Equals Cnephaotachina Brauer and Bergenstamm, 1894.

Eugeniamyia Williston, Can. Ent., vol. 14, p. 80, 1882. 1 species. Type, Rhingia ferruginea FALleN (as rüfa, new species). Equals Brachyopa Meigen, 1822.

Eugnoriste Coquilletr, Prge. Ent. Soc. Washington, vol. 3, p. 321, 1896. 1 species. Type, Eugnoriste occidentalis Coquillett.

Euhybus Coquillett, Proc. U. S. Nat. Mus., vol. 18, p. 437, 1896. 3 species. Type, Hybos purpureus WALKER, the second species, by designation of CoQUILLETT, Proc. Ent. Soc. Washington, vol. 5, 1903, p. 250.

Eujurinia Townsend, Smiths. Misc. Coll., vol. 51, p. 113, 1908. 1 species. Type, Hystricia pollinosa Wulp. Equals Jurinella Brauer and Bergenstami, 1889.

Eulalia Meigen, Nouv. Class. Mouch., p. 21, 1800.* No species. Odontomyia Meigen, 1803, is a change of name. Type, Musca hydroleon Linneus. Syn., Odontomyia Meigen, 1803.

Eulasiona Townsend, Trans. Amer. Ent. Soc., vol. 19, p. 119, 1892.1 species. Type, Eulasiona comstockii Townsend.

Euleia Walker, Ent. Mag., vol. 3, p. 81, 1836. 1 species. Type, Musca cæsio Harris. Equals Acidia Desvoidy, 1830.

Eulonchus Gerstecker, Ent. Zeit. Stettin, vol. 17, p. 359, 1856. 1 species. Type, Eulonchus smaragdinus GersteCKER.

$\checkmark$ Eumacronychia Townsend, Trans. Amer. Ent. Soc., vol. 19, p. 98, 1892. 2 species. Type, Eumacronychia decens Townsend, the first species, by original designation. Equals Hillarella Rondani, 1856.

Eumegaparia Townsend, Smiths. Misc. Coll., vol. 51, p. 58, 1908. 1 species. Type, Megaparia flaveola Coquillett.

$\checkmark$ Eumeros Mejgen, Illiger's Mag., voI. 2, p. 273, 1803. 2 species. Type, Musca segnis Linneus, the first species, by St. Fargead and Serville, who, in Encycl. Meth., vol. 10, pt. 2, 1828, p. 808, selected the second species as type of a new genus, Syritta. Equals Zelima Meigen, 1800.

Eumesembrina Townsend, Smiths. Misc. Coll., vol. 51, p. 50,1908. 1 species (as 2). Type, Mesembrina latreillei Desvoroy. 
Eumetopia Macquart, Dipt. Exot., Suppl. 2, p. 87, 1847. 1 species. Type, Eumetopia rufipes Macquart. Not Eumetopia Westwood, 1837. Equals Eumetopiella HENDEL, 1907.

$\checkmark$ Eumetopia Brauer and Bergenstamm, Denkschr. Kais. Akad. Wiss. Wien, vol. 56. p. 114, 1889. 1 species. Type, Tachina fastuosa Meigen. Not Eumetopia Wesrwoon, 1837. Equals Sphenometopa Townsend, 1908.

Eumetopiella Hendel, Wien. Ent. Zeit., vol. 26, p. 98, 1907. Change of name for Eumetopia Macquart, 1847, not of Westwoon, 1837. Type, Eumetopia rufipes Macquart. Syn., Eumetopia Macquart, 1847, preoccupied.

$\checkmark$ Eumyothyria Townsend, Trans. Amer. Ent. Soc., vol. 19, p. 121, 1892. 1 species. Type, Eumyothyria illinoisensis Towssend.

$\checkmark \quad$ Eurestrophasia Townsend, Trans. Amer. Ent. Soc., vol. 19, p. 133, 1892.1 species. Type, Estrophasia aperta Brauer and Bergenstamm. Equals Ormia Desvoidy, 1830.

$\checkmark$ Euomogenia Townsend, Smiths. Misc. Coll., vol. 51, p. 132, 1908. 1 species. Type, Euomogenia lacteata Townsend. Equals Trichiopoda Latreille, 1829.

$\checkmark$ Eupalamus J жnnicke, Berliner Ent. Zeitschr., vol. 11, p. 86, 1867. 1 species. Type, Cyrtopogon longibarbus Loww (as alpestris, new species). Equals Cyrtopogon LOEW, 1847.

$\checkmark$ Euparyphus Gerst.ecker, Linn. Ent., vol. 11, p. 314, 1857. 1 species. Type, Cyphomyia elegans WIEDEMANN.

$\checkmark$ Eupeitenus Macquart, Dipt. Exot., vol. 1, pt. 1, p. 85, 1838.1 species. Type, Penthetria atra Macquart.

v Eupeleteria Townsend, Smiths. Misc. Coll., vol.51, p. 111, 1908. 3 species. Type, Musca fera Linneus, the first species, by designation of Townsend, Can. Ent., vol. 41, p. 244, 1909. Equals Larvaevora Meigen, 1800.

$\checkmark$ Eupeodes Osten Sacken, Bull. 3, U. S. Geol. Surv., p. 328, 1877. 1 species. Type, Eupeodes volucris Osten SACKen.

$\checkmark$ Euphemia Desvorny, Essai Myod., p. 485, 1830. 4 species. Type, Musca lata Fallen (as pratensis, new species), the first species, by designation of CoquilletT, Journ. N. Y. Ent. Soc., vol. 9, 1901, p. 137. Equals Phaonia Desvorny, 1830.

$\checkmark$ Euphoria Desvordy, Hist. Nat. Dipt., vol. 2, p. 799, 1863. 1 species (as 58). Type, Musca cornicina Fabricius. Not Euphoria Burmeister, 1842. Equals Orthellia Desvoiny, 1863.

$J$ Euphorocera Townsend, Trans. Amer. Ent. Soc., vol. 19, p. 112, 1892. 1 species. Type, Euphorocera tachinomoides Townsend. Equals Phorocera Desvoidy, 1830.

$\checkmark$ Euphrosyne Meigen, Nouv. Class. Mouch., p. 16, 1800.* No species. Macrocera Meigen, 1803, is a change of name. Type, Macrocera lutea Meigen. Syn., Macrocera Meigen, 1803; Geneja Liov, 1864.

$\checkmark$ Euphyto Townsend, Smiths. Misc. Coll., vol. 51, p. 63, 1908. 1 species. Type, Leucostoma subopaca CoquilletT.

$\checkmark$ Eurhinomallota Bigot, Bull. Soc. Ent. France for 1882, p. 67, 1882. 1 species. Type, Eurhinomallota metallica Bigot. Equals Penthesilia Meigen, 1800.

$\checkmark$ Euribia Meigen, Nouv. Class. Mouch., p.36, 1800** No species. Trypeta Meigen, 1803, is a change of name. Type, Musca artemisix Fabricius. Syn., Trypeta Meigen, 1803; Forellia Desvordy, 1830; Spilographa Loew, 1862.

$\checkmark$ Eurigastrina Lioy, Atti Inst. Veneto, ser. 3, vol. 9, p. 1343, 1864. 1 species (as 2).

Type, Tachina vulgaris Fallen. Equals Phryxe Destoidy, 1830.

Eurimyia Bıgot, Bull. Soc. Ent. France for 1883, p. 20, 1883. 1 species. Type, Rhingia lineata FABRICIUs (as rhingoides, new species). Equals Helophilus MeIGeN, 1822.

[Eurina MeIGen, Syst. Beschr. Zweifl. Ins., vol. 6, p. 3, 1830. 2 species (as 3). Type, Eurina lurida Meigen, the second species, by designation of Westwoon, Intr., vol. 2, Synops., 1840, p. 147. Not American.] 
Eurinella Meunier, Bull. Soc. Ent. France for 1893, p. 193, 1893.1 species. Type, Oscinis oscinina Fallen. Equals Madiza Fallen, 1810.

Eurosta Loew, Monogr. Dipt. N. Amer., vol. 3, p. 280, 1873. 3 species. Type, Acinia solidaginis Fiтch, the first species, by present designation.

Eurycephala RöDER, Berliner Ent. Zeitschr., vol. 25, p. 211,1881. 1 species. Type, Eurycephala myoprformis RöDER. Not Eurycephala LAPORTE, 1833. Equals Eurycephalomyia HeNDEL, 1907.

Eurycephalomyia HeNDel, Wien. Ent. Zeit., vol. 26, p. 98, 1907. Change of name for Eurycephala RÖDER, 1881, not of LAPORTE, 1833. Type, Eurycephala myopæformis RöDER. Syn., Eurycephala RöDER, 1881, preoccupied.

Euryceromyia Townsend, Trans. Amer. Ent. Soc., vol. 19, p. 115, 1892. 1 species. Type, Euryceromyia robertsonii Tow nsend.

Eurycnemus Wulp, Tijdschr. Ent., vol. 17, p. 135, 1874. 1 species. Type, Chironomus crassipes Panzer.

Euryneura Schiner, Verh. Zool.-Bot. Ges. Wien, vol. 17, p. 308, 1867. 1 species. Type, Stratiomys fascipennis FABRIcIus.

Euryomma Stein, Ent. Nachr., vol. 25, p. 19, 1899. 1 species. Type, Anthomyia peregrinum MEIGEN (as hispaniense, new species).

Eurytion J Jxnicke, Berliner Ent. Zeitschr., vol. 11, p. 99, 1867. 1 species. Type, Atherix unicolor Curtis (as paradoxus, new species). Equals Ptiolina ZetTerstedt, 1842.

Euscopolia Townsend, Trans. Amer. Ent. Soc., vol. 19, p. 123, 1892. 1 species. Type, Euscopolia dakotensis Townsend.

Eusiphona Coquillett, Rev. Tach., p. 30, 1897. 1 species. Type, Eusiphona mira Coquillett.

Eusisyropa Townsend, Smiths. Misc. Coll., vol. 51, p. 97, 1908. 1 species. Type, Exorista blanda Osten Sack en. Equals Carcelia Desvoidy, 1830.

Eustalomyia Kowarz, Verh. Zool.-Bot. Ges. Wien, vol. 23, p. 461, 1873. 1 species. Type, Musca hilaris Fallen. Syn., Dendrophila Lior, 1864, preoccupied.

Eutachina Brauer and Bergenstamm, Denkschr. Kais. Akad. Wiss. Wien, vol. 56, p. 98, 1889. 1 species. Type, Musca larvarum Linneus. Equals Exorista Meigen, 1803.

Eutanypus Coquillett, Fur Seals N. Pacific Ocean, vol. 4, p. 341, 1899. 1 species. Type, Eutanypus borealis Coquillett.

Eutarsus Loew, Neue Beitr., vol. 5, p. 45, 1857. 1 species. Type, Porphyrops aulicus Meigen.

Euthera Loew, Berliner Ent. Zeitschr., vol. 10, p. 47, 1866.1 species. Type, Euthera tentatrix LoEw.

Euthyneura Macquart, Ann. Soc. Ent. France for 1836, p. 517, 1836.1 species. Type, Euthyneura myrtilli Macquart. Syn., Anthalia Zetterstedt, 1838.

Euthyprosopa Townsend, Trans. Amer. Ent. Soc., vol. 19, p. 106, 1892.1 species. Type, Euthyprosopa petiolata Townsend.

Eutreta Loew, Monogr. Dipt. N. Amer., vol. 3, p. 276, 1873. 2 species. Type, Trypeta sparsa Wiedemann, the first species, by present designation. Syn., Icaria ScHINER, 1868, preoccupied.

Eutrichopoda Townsend, Smiths. Misc. Coll., vol. 51, p. 134, 1908. 1 species. Type, Eutrichopoda nigra Townsend. Equals Trichiopoda Latreille, 1829.

Eutrixa Coquillett, Rev. Tach., p. 39, 1897. 1 species. Type, Clytiomya exile Coquillett.

Euxesta Loew, Berliner Ent. Zeitschr., vol. 11, p. 297, 1868. 14 species. Type, Ortalis notata WieDEMANN, the third species, by present designation.

$\checkmark$ Evanioptera Guerin, Voy. Monde, Coquille, Zool., vol. 2, pt. 2, p. 287, 1838. Change of name for Caloptera GuERIN, 1829, under the false impression that it is preoccupied by Calopteron Laporte, 1836. Type, Caloptera fasciata Guerin. Equals Caloptera Guerin, 1829. 
Evibrissa Rondanı, Dipt. Ital. Prodr., vol. 4, p. 74, 1861. 1 species. Type, Phania obscuripennis MEIGEN.

$\checkmark$ Exaireta Schiner, Verh. Zool.-Bot. Ges. Wien, vol. 17, p. 309, 1867. 1 species. Type, Tylophagus spiriger Wiedemann. Syn., Diphysa Macquart, 1838, preoceupied; Neoexaireta Osten Sacken, 1878.

Exechia Winnertz, Verh. Zool.-Bot. Ges. Wien, vol. 13, p. 879, 1863. 21 species. Type, Tipula fungorum DeGeer, the seventh species, by designation of JoHannsen, Gen. Ins., Dipt., Mycet., 1909, p. 106.

$\checkmark$ Exepacmus Coquillett, Trans. Amer. Ent. Soc., vol. 21, p. 101, 1894.1 species. Type, Exepacmus jolmsoni Coqullett.

w Exocheila Rondan1, Dipt. Ital. Prodr., vol. 2, p. 170, 1857. 1 species. Type, Rhingia ferruginea Fallen. Equals Brachyopa Meigen, 1822.

- Exocheila Rondaxi, Atti Soc. Ital. Sci. Nat., vol. 11, p. 54, 1868 . Change of name for Heterocheila Rondani, 1857, under the mistaken impression that it is preoccupied by 3 somewhat similar names. Type, Heteromyza buccata Fallen. Equals Heteromyza FALLEN, 1820.

$\checkmark$ Exoprosopa Macquart, Dipt. Exot., vol. 2, pt. 1, p. 35, 1840. 41 species. Type, Bibio capucina Fabricius (as pandora Fabricius), the ninth species, by designation of Rondani, Dipt. Ital. Prodr., vol. 1, 1856, p. 162. Lquals Mima Meigen, 1820.

$\checkmark$ Exoptata Coquillett, Can. Ent., vol. 19, p. 13,1887. 1 species. Type, Exoptuta divisa Coquillett.

- Exorista MEıGEN, Illiger's Mag., vol. 2, p. 280, 1803. 1 species. Type, Musca larverum Linneus, Syn., Guerinia Desvoldy, 1830; Strgeria Desvoldy, 1863; Zelleria Desvoldy, 1863; Walkeria Desvoldy, 1863; Zetterstedtia Desvoldy, 1863; Lribea Desvoldy, 1863; Adenia Desvoldy, 1863; Cleodora Desvoldy, 1863; Bigotia Desroiny, 1863; Futilia Dessvordy, 1863; Gaubitia Desvoldy, 1863; Esila Desvoldy, 1863; ITimere Desvoidy, 1863; Eutachina Braver and Bergenstamm, 1889; Chatotachina Brauer and Bergenstamm, 1889; Ptilotachina Brauer and Bergenstamm, 1891; Microtachina Мıк, 1892; Tachinomyin Townsend, 1892. (Exorista of authors equals Carcelia DesiolDy.)

- Exoristoides Coquiliett, Rev. Tach., p. 31, 1897. 2 species. Type, Exoristoides johnsoni Coqunlett, the second species, by original designation.

- Fabricia M Imen, Syst. Beschr. Zweifl. Ins., vol. 7, p. 250,1838. 1 species. Type, Tachina cinerea Fallen (as pacta Meigen). Not Falricia Blainville, 1828. Equals Biomya Rondani, 1856.

Fairmairia Desvoldy, Dipt. Env. Paris, Myop., p. 31, 1853.* 1 species. Type, Myopa morio Meigen. Equals Stomoxoides SCHFFFer, 1766.

- Fallenia Melgẹn, Syst. Beschr. Zweifl. Ins., vol. 7, p. 265, 1838. 2 species. Type, Tachinu lougicornis FalLen, the first species, by present designation. Not Fullenia Meigen, 1820. Equals Campylochæta Rondani, 1859.

Falosoma Lioy, Atti Inst. Veneto, ser. 3, vol. 9, p. 1102, 1864. 5 species. Type, Ephydra aquila FALLEN, the first species, by present designation. Equals Napæa Desvoidi, 1830.

$\checkmark$ Fannia Desvoldy, Essai Myod., p. 567, 1830. 1 species. Type, Musca scalaris Fabricius (as saltatrix, new species). Syn., Philinta Desvoidy, 1830; Aminta Desvolvy, 1830; Iomalomyia Bouché, 1834; Myantha Rondani, 1856.

$\checkmark$ Faunia Desvoiny, Essai Myod., p. 279, 1830. Change of name for Tachina Meigen, 1803, under the mistaken impression that it is preoccupied by Tachinus GravenHorst, 1802. Type, Musca grossa Linneus. Equals Larvævora Meigen, 1800.

$\checkmark$ Faurella Desvoiny, Essai Myod., p. 41, 1830. 1 species. Type, Faurella meridionalis Desvoldy. Equals Peleteria Desvoidy, 1830.

$\checkmark$ Fausta Desvoldy, Essai Myod., p. 62, 1830. 2 species (as 5). Type, Tachina nemorum MEIGEN (the first two and last two supposed species), by designation of Desvoidy, Hist. Nat. Dipt., vol. 1, 1863, p. 158. Equals Ernestia Desvoidy, 1830. 
Fellæa Destoidy, Essai Myod., p. 476, 1830. 4 species (as 5). Type, Fellæa fera Desvoidy, the first species, by designation of CoquilletT, Journ. N. Y. Ent. Soc., vol. 9, 1901, p. 137. Equals Phaonia Desvordy, 1830.

Feltidia Dyar, Proc. Ent. Soc. Washington, vol 7, p. 45, 1905. 3 species. Type, Culex jamaicensis Tнвовацd, the first species, by original designation. Equals Grabhamia THEOBALD, 1903.

Ferdinandea Rondani, Nuovi Ann. Sci. Nat. (Bologna) for 1844, p. 196, 1844. 2 species. Type, Conops cupreus Scopoli, the second species, by designation of RonDanr, Dipt. Ital. Prodr., vol. 1, 1856, p. 51. Syn., Chrysoclamis Rovdani, 1851.

Feronia Leach, On Gen. Species Eproboscideous Ins., p. 4, 1817. 3 species. Type, Feronia spinifera LeACH, the first species, by designation of Speiser, Wien. Ent. Zeit., vol. 18, 1899, p. 202 . Not Feronia Latreille, 1817. Equals Olfersia Wiedemann, 1830.

Fidena Walker, Ins. Saund., Dipt., p. 8, 1850. 4 species. Type, Pangonius leucopogon Wiedemans, the first species, by present designation. Equals Pangonius IATREILLE, 1802.

Flabellifera Meigen, Nouv. Class. Mouch., p. 13, 1800.* No species. Ctenophora Meigen, 1803, is a change of name. Type, Tipula atrata Linneus. Syn., Ctenophora Meigen, 1803; Tanyptera Latreille, 1804; Xiphura Brullé, 1832.

Flebotomus Rondani, Mem. Prima Serv. Dipt. Ital., p. 12, 1840** 1 species. Type, Bibio papatasii -Scopoli. Syn., Cyniphes Costa, 1843; Hæmasson Loew, 1844. Forcipomyia Meigen, Syst. Beschr. Zweifl. Ins., vol. 1, p. 73, 1818. 2 species. Type, Ceratopogon ambiguus Meigen, the first species, by present.designation. Equals Culicoides Latreille, 1809.

$\checkmark$ Forellia Desvordy, Essai Myod.; p. 760, 1830. 3 species. Type, Musca artemisix Fabricius (as onopordi, new species), the first species, by designation of Rondani, Bull. Soc. Ent. Ital., vol. 2, 1870, p. 7. Equals Euribia Meigen, 1800.

$\checkmark$ Frauenfeldia EgGer, Verh. Zool.-Bot. Ges. Wien, vol. 15, p. 297, 1865. 1 species. Type, Tachina rubricosa Meigen. Equals Tricogena Rondani, 1856.

Freræa Desvoldy, Essai Myod., p. 285, 1830. 1 species. Type, Freræa gagatea Desvoidy. Syn., Gymnophania Brauer and Bergenstamm, 1889.

Frivaldskia Schiner, Wien. Ent. Monatschr., vol. 5, p. 142, 1861. Change of name for Fallenia Meigen, 1838, not of Meigen, 1820. Type, Tachina longicornis Fallen. Equals Campylochæta Rondani, 1859.

Frontina Meigen, Syst. Beschr. Zweifl. Ins., vol. 7, p. 247, 1838. 3 species. Type, Tachina læta Meigen, the first species, by designation of Desvordy, Hist. Nat. Dipt., vol. 1, 1863, p. 580. 'Syn., Achætoneuru Brauer and Bergenstamm, 1891; Parafrontinc Brauer and Bergenstami, 1893.

- Fucellia Desvordy, Ann. Soc. Ent. France for 1841, p. 269, 1841. 1 species. Type, Halithea maritima Haliday (as arenaria, new species). Syn., Halithea HaliDAY, 1838, preoccupied.

Fucomyia Haliday, Ann. Nat. Hist., vol. 2, p. 186, 1838. 3 species. Type, Colopa eximia Stenhammer (as Musca frigida Fabricius), the first species, by designation of Westwood, Intr., vol. 2, Synops., 1840, p. 144. Equals Cœlopa Meigen, 1830.

Fungivora Meigen, Nouv. Class. Mouch., p. 16, 1800.* No species. Mycetophila Meigen, 1803, is a change of name. Type, Mycetophila agarici Olivier. Syn., Mycetophila Meigen, 1803; Mycetina Rondani, 1856; Mycozetra Rondani, 1861.

Fungobia Lroy, Atti Inst. Veneto, ser: 3, vol. 9, p. 1114, 1864. 1 species. Type, Borborus nitidus Meigen. Equals Copromyza Fallen, 1810.

Furcinerva Rondani, Nuovi Ann. Sci. Nat. (Bologna) for 1846, pp. 368, 369, 1846. No species. Founded on the union of four genera: Zygoneura Meigen, Lestremia Macquart, Mimosciara Rondani, and Catocha Haliday. The second and third are congeneric, and the present genus may take the type of the third. Type, Mimosciara molobrina Rondani. Equals Lestremia MacQUaRT, 1826.

Proc.N.M.vol.37-10—35 
Furcomyia Mleigen, Syst. Beschr. Zweifl. Ins., vol. 1, p. 134, 1818. 1 species. Type, Limonia lutea Melgen. Syn., Dicranomyia Stephexs, 1829; Glochina Melgen, 1830; Siagona Melgex, 1830; Numantia B1got, 1854.

$\checkmark$ Furina J envicke, Abh. Sencken. Ges., vol. 6, p. 318, 1867. 1 species. Type, Limnobia rufithorax Wiedenans. Equals Gnophomyia Osten Sacken, 1860.

- Futilia Desvoldy, Hist. Nat. Dipt., vol. 1, p. 1049, 1863. 1 species (as 23). Type, Tachina simulans Melgex. Equals Exorista Melges, 1803.

$\checkmark$ Gædiophana Brauer and Bergenstamu, Denkschr. Kais. Akad. Wiss. Wien, vol. 60, p. 201, 1893. 1 species. Type, Gidiophana atra Brauer and Bergenstamu.

v Gædiopsis Brauer and Bergengtam, Denkschr. Kais. Akad. Wiss. Wien, vol. 58, p. $336,1891.1$ species. Type, Gicdiopsis mexicana Brauer and Bergenstamm. Syn., Poliophrys Townsend, 1908.

- Galactomyia Townsend, Smiths. Mise. Coll., vol. 51, p. 135, 1908. 1 species (as 3). Type, Thereva lanipes Fabricius. Equals Trichiopoda Latreille, 1829.

- Gasterophilus Leach, On Arrang. Oestrideous Ins., p. 2, 1817. 3 species. Type, Estrus intestinalis De GEER (as Wstrus equi CLARK), the first species, by designation of Curtis, Brit. Ent., 1826, p. 138. Syn., Gastrus Meigen, 1824; Enteromyza RoNDAN1, 1857.

$\checkmark$ Gastrichelius Rondan1, Dipt. Ital. Prodr., vol. 1, p. 156, 1856. 1 species. Type, Triclis oliraceus Loew (as halictivorus, new species). Equals Triclis Loew, 1851.

- Gastrolepta Lioy, Atti Inst. Veneto, ser. 3, vol. 9, p. 909, 1864. 1 species. Type, Musea coarctata Fallen. Equals Hylemya Desvoidy, 1830.

Gastrops Williston, Kansas Univ. Quart., vol. 6, p. 3, 1897. 1 species. Type, Gastrops niger iWilliston.

$\checkmark$ Gastrus Melgen, Syst. Beschr. Zweifl. Ins., vol. 4, p. 174, 1824. Change of name for Gasterophilus LeACH, 1817; reason for the change not mentioned. Type, Estrus intestinalis De Geer. Equals Gasterophilus Leach, 1817.

Gaubilia Desvoldy, Hist. Nat. Dipt., vol. 1, p. 1062, 1863.1 species. Type, Tachina simulans Melgen (as dominula, new species). Equals Exorista Melgen, 1803.

$\checkmark$ Gaurax Loew, Berliner Ent. Zeitschr., vol. 7, p. 35, 1863. 1 species. Type, Gaurax festivus LoEw.

$\checkmark$ Geminaria Coquillett, Trans. Amer. Ent. Soc., vol. 21, p. 109, 1894. 2 species. Type, Lordotus canalis Coquilletr, the first species, by original designation.

- Geneja Lior, Atti Inst. Veneto, ser. 3, vol. 9, p. 229, 1864. 6 species. Type, Macrocera fasciata Melgen, the third species, by present designation. Equals Euphrosyne Meigen, 1800.

$\checkmark$ Geomyza Fallen, Spec. Ent. Meth. Exh., p. 18, 1810. 1 species. Type, Musca combinata Linneus. Syn. Balioptera Loew, 1865.

$\checkmark$ Geosargus Bezz1, Wien. Ent. Zeit., vol. 26, p. 53, 1907. Change of name for Sargus Fabricius, 1798, not of Walbridge, 1792. Type, Musca cuprarius Linseus. Syn., Sargus Fabricius, 1798, preoceupied.

$\checkmark$ Geranomyia Haliday, Ent. Mag., vol. 1, p. 154, 1833. 1 species. Type, Geranomyia unicolor Halway. Syn., Limnobiorhynchus Westwood, 1835; Aporosa MaCQUART, 1838; Plettusa PhilıPP1, 1865.

$\checkmark$ Geron Meigen, Syst. Beschr. Zweifl. Ins., vol. 2, p. 223, 1820. 1 species (as 2). Type, Bombylius hybridus Meigen.

$\checkmark$ Gervasia Desvo1dy, Hist. Nat. Dipt., vol. 2, p. 36, 1863. 1 species. Type, Tachina nigripes Fallen (as piniarix Hartig). Equals Lydella Desvoldy, 1830.

$\checkmark$ Giardomyia Felt, 23d Rep. State Ent. N. Y., p. 405, 1908. 6 species. Type, Cecidomyia photophila Fest, the third species, by original designation.

$\checkmark \quad$ Ginglymyia Townsend, Trans. Amer. Ent. Soc., vol. 19, p. 118, 1892. 1 species. Type, Ginglymyia acrirostris Townsend. 
Glabrinus Rondani, Dipt. Ital. Prodr., vol. 1, p. 132, 1856.1 species. Type, Syrphus subsultans Fabricius (as murorum, new species). Equals Mosillus Latreille, 1805.

Glaphyroptera Winnertz, Verh. Zool.-Bot. Ges. Wien, vol. 13, p. 781, 1863.11 species. Type, Lein fascipennis MeIgen, the first species, by present designation. Equals Leia MeIgen, 1818.

Glaucophana Brauer and Bergenstamm, Denkschr. Kais. Akad. Wiss. Wien, vol. 58, p. 354, 1891.1 species. Type, Glaucophana amasiz Brauer and Bergensta mm. Equals Neæra Desvoldy, 1830.

Glaurotricha Thомson, Kongl. Sven. Freg. Eugenies Resa, Dipt., p. 493, 1868.1 species. Type, Glaurotricha muscaria Thomson. Equals Phalacromya Rondani, 1848.

Glochina Meigen, Syst. Beschr. Zweifl. Ins., vol. 6, p. 280, 1830. 1 species. Type, Glochina sericata Meigen. Equals Furcomyia MeIGen, 1818.

Glutops Burgess, Proc. Bost. Soc. Nat. Hist., vol. 19, p. 321, 1878. 1 species. Type, Glutops singularis Burgess.

$\checkmark$ Gamptopsilopus Aldrich, Kansas Univ. Quart., vol. 2, p. 48, 1893.4 species. Type, Psilopus scintillans Loww, the first species, by present designation. Equals Sciapus ZeLler, 1842.

Gnophomyia Osten Sacken, Proc. Acad. Nat. Sci. Phila. for 1859, p. 223, 1860. 2 species. Type, Gnophomyia tristissima Osten SACKEn, the second species, by present designation. Syn. Furina $\mathrm{J}_{£ \text { NNICKe, }} 1867$.

Gnoriste MeIGeN, Syst. Beschr. Zweifl. Ins., vol. 1, p. 243, 1818. 1 species. Type, Gnoriste apicalis MeIGEN.

$\checkmark$ Gonia MLIG EN, Illiger's Mag., vol. 2, p. 280, 1803. No species. In his Syst. Beschr., vol. 5,1826 , pp. 2 to 7,13 species. Type, Musca capitata De Gerr, the second species, by designation of Curtis, Brit. Ent., 1835, p. 533. Equals Salmacia Meigen, 1800. Goniglossum Rondani, Dipt. Ital. Prodr., vol. 1, p. 110, 1856. 1 species. Type, Trypeta wiedemanni Meigen. Fquals Orellia Desvoidy, 1830.

$\checkmark$ Goniochæta Townsend, Trans. Amer. Ent. Soc., vol. 18, p. 351, 1891. 1 species. Type, Goniochrta plagioides Townsend.

Goniomima Townsend, Smiths. Mise. Coll., vol. 51, p. 105, 1908. 1 species. Type, Belvosia luteola Coquillett.

Gonioneura Rondani, Bull. Soc. Ent. Ital., vol. 12, p. 5, 1880. 1 species. Type, Gonioneura bisangula Rondani. Equals Leptocera Olivier, 1813.

- Goniops Aldrich, Psyche, vol. 6, p. 236, 1892. 1 species. Type, Pangonius chrysocoma Osten SACKen (as hippoboscoides, new species).

$\checkmark$ Gonioscelis Schiner, Verh. Zool.-Bot. Ges. Wien, vol. 16, p. 670, 1866. 1 species. Type, Dasypogon hispidus Wiedemann. Equals Stenopogon Loew, 1847.

ᄂ Gonirhyncis Rondani, Dipt. Ital. Prodr., vol. 1, p. 58, 1856. 1 species. Type, Myopa occulta Meigen (as dispar, new species). Equals Stomoxoides Sch afFEr, 1766.

Gonomyia Meigen, Syst. Beschr. Zweifl. Ins., vol. 1, p. 146, 1818. 1 species. Type, Limnobia tenella Meigen. Syn., Taplirosia Rondani, 1856.

Gonypes Latreille, Hist. Nat. Crust. et Ins., vol. 14, p. 309, 1805. 1 species. Type, Asilus cylindricus De Geer (as tipuloides Linneus). Equals Leptogaster Meigen, 1803.

Grabhamia Theовацd, Monogr. Culic., vol. 3, p. 243, 1903.10 species. Type, Culex jamaicensis Theовацd, the third species, by designation of Felt, Bull. 323, N. Y. State Museum, 1904, p. 391b. Syn., Feltidia Dyar, 1905; Ceratocystia Dyar and $\mathrm{K}_{\mathrm{NAB}}, 1906$.

Grallopoda Rondani, Nuovi Ann. Sci. Nat. (Bologna), ser. 3, vol. 2, p. 180, 1850. 1 species. Type, Musca lasciva Fabricius (as Calobata albimana Macquart). 
$\sqrt{\text { Graphomya Desvoidy, Essai Myod., p. 403, 1830. } 3 \text { species (as 5). Type, Musca }}$ maculata Scopoli, the first species, by designation of Rondani, Dipt. Ital. Prodr., vol. 1, 1856, p. 91. Syn., Curtonevra Macquart, 1834.

$\checkmark$ Grassia Theobald, Journ. Trop. Med., vol. 5, p. 181, June 16, 1902. 1 species. Type, Anopheles rossii Giles. Not Grassia Fisch, 1885. Equals Myzomyia BlanchARD, July 4, 1902.

Griphoneura Schiner, Reise Novara, Zool., vol. 2, p. 281, 1868. 1 species. Type, Lauxania imbuta WIEDEMANN.

V Guerinia Desvoidy, Essai Myod., p. 196, 1830. 1 species (as 6). Type, Guerinia festiva Desvoidy. Equals Exorista Meigen, 1803.

$\checkmark$ Gymnocheta Desvoroy, Essai Myod., p. 371, 1830. 1 species. Type, Tachina viridis FALLEN.

- Gymnoclytia Brauer and Bergenstamm, Denkschr. Kais. Akad. Wiss. Wien, vol. 60 , p. 157, 1893. 1 species. Type, Cistogaster immaculata MaCquart (as divisa Loew). Equals Cistogaster Latreille, 1829.

- Gymnodia Desvordy, Hist. Nat. Dipt., vol. 2, p. 635, 1863. 1 species. Type, Anthomyia polystigma Melgen (as pratensis, new species). Equals Limnophora DesvoIDy, 1830.

- Gymnogaster Lioy, Atti Inst. Veneto, ser. 3, vol. 9, p. 989, 1864. 1 species. Type, Anthomyja dissecta Meigen. Equals Pegomya Desvoidy, 1830.

- Gymnometopa Coquillett, Proc. Ent. Soc. Washington, vol. 7, p. 183, 1906. 4 species. Type, Stegomyia mediorittata Coqunllett, the first species, by original designation.

C Gymnomma Wulp, Biol. Centr.-Amer., Dipt., vol. 2, p. 38, 1888. 1 species. Type, Gymnomma nitidiventris IVuLP.

- Gymnopa Fallen, Oscinides Sveciæ, p. 10, 1820. 1 species. Type, Syrphus subsultans Fabricius. Equals Mosillus Latreille, 1805.

- Gymnopareia Brauer and Bergenstamm, Denkschr. Kais. Akad. Wiss. Wien, vol. 56, p. 103, 1889.1 species. Type, Tachina crassicornis Meigen. Equals Actia DesvoIDY, 1830.

Gymnophania Brauer and Bergenstamm, Denkschr. Kais. Akad. Wiss. Wien, vol. 56, p. 143, 1889. 1 species. Type, Gymnophania nigripennis BraUer and Bergenstamm. Equals Freræa Desvoidy, 1830.

Gymnophora MAcquart, Hist. Nat., Dipt., vol. 2, p. 631, 1835. 1 species. Type, Phora arcuata Meigen.

$\checkmark$ Gymnophtalma Lioy, Atti Inst. Veneto, ser. 3, vol. 9, p. 1341, 1864. 1 species. Type, Tachina crassicornis Meigen. Equals Actia Desvoidy, 1830.

Gymnoprosopa Townsenn, Trans. Amer. Ent. Soc., vol. 19, p. 108, 1892. 1 species (as 3). Type, Gymnoprosopa polita Townsend. Equals Hilarella Rondani, 1856.

Gymnopsis RondanI, Dipt. Ital. Prodr., vol. 3, p. 90, 1859. 1 species. Type, Tachina chalconota MeIgen. Equals Macquartia Desvoidy, 1830.

$\checkmark$ Gymnoptera Lioy, Atti Inst. Veneto, ser. 3, vol. 10, p. 79, 1864. 1 species. Type, Phora vitripennis Meigen. Equals Hypocera Lioy, 1864.

$\checkmark$ Gymnopternus Loew, Neue Beitr., vol. 5, p. 10, 1857. 28 species. Type, Dolichopus cupreus FALLEN, the twenty-first species, by present designation.

$\checkmark$ Gymnosoma Meigen, Illiger's Mag., vol. 2, p. 278, 1803. 1 species. Type, Musca rotundata Linnzus. Equals Rhodogyne MeIgen, 1800.

Gymnostyla Lioy, Atti Inst. Veneto, ser. 3, vol. 9, p. 1000, 1864. 1 species. Type, Musca cucularia Linnжus. Equals Elgiva Meigen, 1838.

$\checkmark$ Habropogon Loew, Linn. Ent., vol. 2, p. 463, 1847. 2 species. Type, Dasypogon exquisitus MeIgen, the first species, by present designation. Syn., Dactiliscus RonDAN1, 1856.

$\checkmark$ Hadromyia Wrliston, Can. Ent., vol. 14, p. 78, 1882. 1 species. Type, IIadromyia grandis Williston. Equals Pocota St. Fargeau and Serville, 1828. 
Hadrus Perty, Delect. Anim., p. 182, 1834. 5 species. Type, Hrmatopota crassipes Fabricius, the first species (as Tabanus lepidotus W1EDEMANN), by present designation. Syn., Lepiselaga Macquart, 1838.

Hæmagogus Williston, Trans. Ent. Soc. Lond. for 1896, p. 271, 1896. 1 species. Type, Hrmagogus splendens Williston.

Hæmasson Loew, Ent. Zeit. Stettin, vol. 5, p. 115, 1844. 1 species. Type, Bibio papatasii Scopoli (as minutus, new species). Equals Flebotomus Rondani, 1840.

Hæmatobia St. Fargeau and Serville, Encycl. Meth., vol. 10, pt. 2, p. 499, 1828. 2 species. Type, Conops irritans Linneus, the second species, by designation of Westwood, Intr., vol. 2. Synops., 1840, p. 140; Syn., Lyperosia Rondanl, 1856; Priophora DesvoIDy, 1863.

Hæmatopata Meigen, Illiger's Mag., vol. 2, p. 267, 1803. 1 species. Type, Tabanus plumilis TINanes. Equals Chrysozona MeIgen, 1800.

$\checkmark$ ITimobora Curtis, Brit. Ent., p. 14, 1824. 1 species. Type, Pediculis cervi LiNNeUs. Equals Lipoptena Nrtzsch, 1818.

Halidaya Rondani, Dipt. Ital. Prodr., vol. 1, p. 117, 1856. 1 species. Type, Themira pilosa Desvordy (as setosa Desvoidy). Equals Themira Desvoidy, 1830.

Halithea Haliday, Ann. Nat. Hist., ser. 1, vol. 2, p. 185, 1838. 2 species. Type, Scatomyza fucorum Fallen, the first species, by designation of Coquillett, Journ. N. Y. Ent. Soc., vol. 9, 1901, p. 137. Not Halithea SAVIGNY, 1817. Equals Fucellia Desvoidy, 1841.

Halterophora Rondan1, Dipt. Ital. Prodr., vol. 4, p. 10, 1861. Change of name for Ceratitis MacLeay, 1829, under the mistaken impression that it is preoccupied by Ceratites HaAn, 1825. Type, Trypeta capitata Wiederiann. Equals Ceratitis MaCLEAY, 1829.

Hammerschmidtia Schummel, Isis von Oken ior 1834, p. 740, 1834. 1 species. Type, Rhingia ferruginea FALLEN (as vittrir, new species). Equals Brachyopa MEIGEN, 1822.

Hammomyia Rondan1, Dipt. Ital. Prodr., vol. 6, p. 13, 1877. 1 species. Type, Leucophora cinerea Desvoidy (as Aricia albescens ZetTerstedt). Equals Leucophora Desvoidy, 1830.

Haplegis Loew, Zeitschr. Ent. Breslau, vol. 15, p. 22, 1866.* 3 species. Type, Chlorops diadema Meigen, the first species, by present designation. Equals Cryptonevra Lioy, 1864.

Harrisia MeIgen, Syst. Beschr. Zweifl. Ins., vol. 7, p. 260, 1838. 3 species. Type, Tachina ænea MEIGEN, the third species (as Tachina simplicitarsis ZetTERstedT), by designation of Rondani, Dipt. Ital. Prodr., vol. 1, 1856, p. 65. Equals Lydina DrsyolDy, 1830.

Hartigia Desvordy, Hist. Nat. Dipt., vol. 2, p. 521, 1863. 1 species (as 17). Type, Sarcophaga dissimilis MeIgen. Syn., Erichsonia Desvoidy, 1863; Mulsantia Desvoidy, 1863; Helicobia Coquillett, 1895.

Hebecnema Schnabl, Hor. Soc. Ent. Ross., vol. 23, p. 331, 1889. 5 species. Type, Anthomyia umbratica Meigex, the first species, by designation of Coquillett, Journ. N. Y. Ent. Soc., vol. 9, 1901, p. 137. Equals Hydrophoria Desvoidy, 1830.

Hebia Desvoidy, Essai Myod., p. 98, 1830. 1 species. Type, Hebia flavipes Desvoidy. Equals Leskia Desvordy, 1830.

Helcomyza CurTis, Brit. Ent., p. 66, 1825. 1 species. Type, Helcomyza ustulata Curtis. Syn., Actora Meigen, 1826.

Helea Meigen, Nouv. Class. Mouch., p. 18, 1800.* No species. Ceratopogon Meigen, 1803, is a change of name. Type, Ceratopogon communis Meigen. Syn., Ceratopogon Meigen, 1803; Palpomyia Meigen, 1818; Apogon Rondani, 1856; Alasion Rondani, 1857 ; Atrichopogon KiefFer, 1906. 
Heleodromia Haliday, Ent. Mag., vol. 1, p. 159, 1833. 4 species. Type, Heleodromia immaculata Halidar, the first species, by designation of CurTis, Brit. Ent., 1834, p. 519. Syn., Sciodromia Halidar, 1840; Microcera Zetterstedt, 1838.

$\checkmark$ Heleomyza Fallen, Spec. Ent. Meth. Exh., p. 19, 1810.1 species. Type, Musca serrata Linneus. Syn., Leria Desvoidy, 1830; Orbellia Desvoidy, 1830; Blephariptera Macquart, 1835. (Heleomyza of authors equals Suillia Desvoidy.)

$\checkmark \quad$ Helicobia Coquillett, Proc. Acad. Nat. Sei. Phila. for 1895, p. 317, 1895. 1 species. Type, Sarcophaga helicis Townsend. Equals Hartigia Desvordy, 1863.

r Heligmonevra Bigот, Thompson's Arch. Ent., vol. 2, p. 356, 1858. 1 species. Type, Heligmonerru modesta Bigot. Syn., Mochtherus Loew, 1849, preoccupied; Neomochtherus OSTEN SACKen, 1878.

$\checkmark$ Helina Desvordr, Essai Myod., p. 493, 1830. 6 species (as 7). Type, Anthomyia pertusu Meigen (as euphemioidea, new species), the first species, by designation of Coquillett, Journ. N.Y. Ent. Soc., vol. 9, 1901, p. 137. Equals Mydæa Desvoidy, 1830.

$\checkmark$ Heliophilus Meigen, Illiger's Mag., vol. 2, p. 273, 1803. 1 species. Type, Musca sylvarum Linnæus. Equals Zelima Meigen, 1800.

- Helius St. Fargeau and Seriville, Encycl. Meth., vol. 10, pt. 2, p. 832, 1828. Change of name of Megarhina St. Fargeau and Serville, 1828, on account of Magarhinus Desvoidy, 1827. Type, Limnobia longirostris MeIgen. Equals Megarhina St. Fargead and Serville, 1828.

Helobic St. Fargeau and Serville, Encycl. Meth., vol. 10, pt. 2, p. 585, 1828. 1 species. Type, Limonia hylrida M kigen (as Limnobia punctipennis MeIgen). Not Helobia Stephens, 1827. Equals Symplecta Meigen, 1830.

$\checkmark$ Helophilus Meigen, Syst. Beschr. Zweifl. Ins., vol. 3, p. 368, 1822. 7 species. Type, Musca pendula Linnæus, the sixth species, by designation of Curris, Brit. Ent., 1832, p. 429. Syn., Lejops Rondani, 1857; Mesembrius Rondani, 1857; Anasimyia SCHINer, 1864; Eurimyia Bigot, 1883.

v [Hemerodromia Mieigen, Syst. Beschr. Zweifl. Ins., vol. 3, p. 61, 1822. 9 species. Type, Empis melanocephala FABricius (as Tachydromia mantispa PAnzer), the fifth species, by designation of Westwood, Intr., vol. 2, Synops., 1840, p. 132. Syn., Chelipoda Macquart, 1823; Phyllodromia Zetterstedt, 1837; Lepidomya Bigot, 1857; Thammodromia MıK, 1886. ' Not American.] (Hemerodromia of authors equals Chelifera Macquart.)

Hemeromyia Coquillett, Journ. N. Y. Ent. Soc., vol. 10, p. 190, 1902. 1 species. Type, Hemeromyia obscura Coquillett.

- Hemiargyru Townsend, Smiths. Misc. Coll., vol. 51, p. 88, 1908. 1 species. Type, Hemiargyra nigra Townsend. Equals Metadoria Brauer and Bergenstamm, 1893.

$\checkmark$ Hemichlora WulP, Biol. Centr.-Amer., Dipt., vol. 2, p. 303, 1896.1 species. Type, Cyrtonerra vittigera Bigot.

Hemipenthes Loww, Berliner Ent. Zeitschr., vol. 13, p. 28, 1869. 1 species (as 2). Type, Musca morio Linn wus. Equals Villa Lioy, 1864.

v Hemithra Desvordy, Hist. Nat. Dipt., vol. 1, p. 318, 1863. 1 species. Type, Tachina erythrostoma Hartig. Equals Phryxe Desvoidy, 1830.

- Hemithrixion Brauer and Bergenstamm, Denkschr. Kais. Akad. Wiss. Wien, vol. 58, p. 357, 1891 . 1 species. Type, Hemithrixion astriforme Brauer and Bergenstamm.

v Hemyda Desvoidy, Essai Myod., p. 226, 1830. 1 species. Type, Hemyda aurata Desvoidy. Syn., Ancylogaster Bigot, 1884.

$\checkmark$ Henicomyia Coquillett, Journ. N. Y. Ent. Soc., vol. 6, p. 187, 1898. 1 species. Type, Henicomyia hubbardii CoQuILleTt.

$\checkmark \quad$ Henops Meigen, Klass. Beschr. Zweifl. Ins., p. 150, 1804. 1 species (as 2). Type, Musca gibbosa Linneus. Equals Ogcodes Latreille, 1796.

$\checkmark \quad$ Herbina Desvordy, Essai Myod., p. 698, 1830. 2 species. Type, Herbina suillioidea Desvoidy. Equals Suillia Desvordy, 1830. 
Herbstia Desvordy, Ann. Soc. Ent. France for 1851, p. 184, 1851. 1 species. Type, Herbstia tibialis Desvordy. Equals Actia Desvordy, 1830.

Hercostomus Loew, Neue Beitr., vol. 5, p. 9, 1857. 3 species. Type, Sybistroma longiventris Loew, the first species, by original designation.

Heringia Rondan1, Dipt. Ital. Prodr., vol. 1, p. 53, 1856. 1 species. Type, Pipiza heringi Zetterstedt. Equals Pipiza Fallen, 1810.

Hermetia Latreille, Hist. Nat. Crust. et Ins., vol. 14, p. 338, 1805.1 species. Type, Musca illucens Linnsus.

Hermione Meigen, Nouv. Class. Mouch., p. 22, 1800.* No species. Oxycera Mrigen, 1803, is a change of name. Type, Musca hypoleon Linneus. Syn., Oxycera Meigen, 1803.

$\checkmark$ Hesperempis Melander, Ent. News, vol. 17, p. 377, 1906. 1 species. Type, Ragas mabeli Melander.

Hesperinus WALKer, List Dipt. Brit. Mus., vol. 1, p. 81, 1848. 1 species. Type, Hesperinus brevifrons WALkER. Syn., Spodius Loew, 1858.

Hesperodes Coquilletr, Ent. News, vol. 11, p. 429, 1900. 1 species. Type, Hesperodes johnsoni CoquILLETT.

Hesperomyia Brauer and Bergenstamm, Denkschr. Kais. Akad. Wiss. Wien, vol. 56, p. 114, 1889. 1 species. Type, Hesperomyia erythrocera Brauer and BergenSTAMM.

Hesyquillia Desvordy, Essai Myod., p. 708, 1830. 2 species. Type, Musca umbrarum FABRIcius (as lugubris, new species), the first species, by present designation. Equals Platystoma Meigen, 1803.

Heteracanthia Macquart, Dipt. Exot., Suppl. 4, p. 43, 1850. 1 species. Type, Heteracanthia ruficornis MACQUART.

$\checkmark$ Heteralonia Rondani, Arch. Zool., vol. 3, p. 57, 1864. 1 species. Type, Exoprosopa occulta Macquart. Equals Mima Meigen, 1820.

Heterocheila Rondani, Dipt. Ital. Prodr., vol. 2, p. 13, 1857. Change of name for Heterostoma Rondani, 1856, preoccupied. Type, Heteromyza buccata Fallen. Equals Heteromyza FaLlex, 1820.

Heteromyia SAY, Amer. Ent., vol. 2, p. 79, 1825, 1 species. Type, Heteromyia fasciata $\mathrm{S}_{\mathbf{A Y}}$.

Heteromyza Fallex, Heter. Sveciæ, p. 1, 1820. 2 species. Type, Heteromyza buccata Fallen, the second species, by Desvoidy, Essai Myod., 1830, p. 655, who took the first species as type of a new genus, Thelida. Syn., Heterostoma Rondani, 1856; Heterocheila Rondani, 1857; Edoparea Loew, 1862; Exocheila Rondani, 1868.

$\checkmark$ Heteroneura Fallex, Spec. Ent. Meth. Exh., p. 7, 1810. 1 species. Type, Heteroneura leptiformis Fallen. Equals Callomyia Meigen, 1804.

- Heteroneura FAllen, Agromyzides Sveciæ, p. 2, 1823. 4 species (as 3 and 1 variety). Type, Heteroneura albimana Meigen (the variety $b$, of the second species), by designation of CurTis, Brit. Ent., 1838, p. 721. Not Heteroneura Falden, 1810. Equals Clusiodes Coquillett, 1904.

$\checkmark$ Heteronycha Arribalzaga, Rev. Mus. La Plata, vol. 1, p. 397, 1891.1 species. Type, Culex ærstuans Windemann (as dolosa, new species). Equals Culex Linneus, 1758.

Heteropogon Loww, Linn. Ent., vol. 2, p. 488, 1847. 3 species. Type, Dasypogon manicatus Meigen, the first species, by designation of BACK, Trans. Amer. Ent. Soc., vol. 35, p. 318, 1909. Syn., Anisopogon Loew, 1874.

$\checkmark$ Heteroptera Macquart, Hist. Nat., Dipt., vol. 2, p. 570, 1835. 1 species. Type, Copromyza pusilla Fallen. Equals Cypsela Meigen, 1800.

Heteropterina Macquart, Ann. Soc. Ent. France for 1854, p. 426; 1854.1 species. Type, Miltogramma heteroneura Meigen. Equals Taxigramma Perris, 1852.

$\checkmark$ Heterostoma Rondani, Dipt. Ital. Prodr., vol. 1, p. 104, 1856. 1 species. Type, Heteromyza buccata Fallen. Not Heterostoma Hartman, 1843. Equals Heteromyza FALLEN; 1820. 
$\checkmark$ Heterostylum Macquart, Dipt. Exot., Suppl. 3, p. 35, 1848. 1 species. Type, Heterostylum flanum Macquart. Syn., Comastes Osten Sacken, 1877.

Hexacantha Meigex, Illiger's Mag., vol. 2, p. 264, 1803. 2 species. Type, Musca chalybeata Forster (as Stratiomys sexdentata Fabricius), the second species, by tautonomy. Equals Beris Latreille, 1802.

$\checkmark \quad$ Hexachæta Loww, Monogr. Dipt. N. Amer., vol. 3, p. 219, 1873. 3 species. Type, Trypeta eximia Wredemann, the first species, by present designation.

$\checkmark$ Hexamitocera BeCKer, Berliner Ent. Zeitschr,, vol. 39, p. 107, 1894. 1 species. Type, Cordilura loxocerata FALLen.

$\checkmark$ Hexatoma Latreille, Gen. Crust. et Ins., vol. 4, p. 260, 1809. 1 species. Type, Hexatoma nigra Latreille. Syn., Nematocera Meigen, 1818; Anisomera Wiedemann, 1818.

- Hiatus Cresson, Trans. Amer. Ent. Soc., vol. 32, p. 280, 1906. 1 species. Type, Hiatus fulvipes Cresson.

$\checkmark$ Hilara MeIgen, Syst. Beschr. Zweifl. Ins., vol. 3, p. 1, 1822. 21 species. Type, Empis maura Fabricius, the second species, by designation of CurTis, Brit. Ent., 1826 , p. 130.

Hilarella Rondani, Dipt. Ital. Prodr., vol. 1, p. 70, 1856. 1 species. Type, Mittogramma hilarella Zetterstedt. Syn., Eumacronycha Townsend, 1892; Gymnoprosopa Townsend, 1892.

$\checkmark$ Hilarimorpha Schiner, Wien. Ent. Monatschr., vol. 4, p. 54, 1860.1 species. Type, Hilarimorpha singulteris Schiner.

- Himantostoma Loew, Berliner Ent. Zeitschr., vol. 7, p. 321, 1863. 1 species. Type, Himantostoma sugens LoEw. Not Himantostoma Agassiz, 1862.

- Himeru Desvoidy, Hist. Nat. Dipt., vol. 1, p.112t, 1863. 1 species (as 4). Type, Guerinia festira Destoidy. Equals Exorista Meigen, 1803.

$\checkmark$ Himerœssa Loew, Monogr. Dipt. N. Amer., vol. 3, p. 85, 1873.1 species. Type, Himercessa pretiosa LoEw.

- Hippelates Loww, Berliner Ent. Zeitschr., vol. 7, p. 36, 1863. 2 species. Type, Hippelates plebejus Loww, the second species, by present designation. Syn., Siphomyia Williston, 1896.

. Hippobosca Linnæus, Syst. Nat., 10th ed., p. 607, 1758. 4 species. Type, Hippobosca equina Linneus, the first species, by designation of Latreille, Consider. Général., 1810, p. 4t4. Syn., Nirmomyia Nitzsch, 1818.

$\checkmark$ Hirmoneura Meigen, Syst. Beschr. Zweifl. Ins., vol. 2, p. 132, 1820. 1 species. Type, Hirmoneura obscura Wiedenann. Syn., Parasymmictus Bigot, 1879.

- Histiodroma Schiner, Verl. Zool.-Bot. Ges. Wien, vol. 17, p. 308, 1867. 1 species. Type, Sargus inermis Wiedemann.

$\checkmark$ Holcocephala J ÆNnicke, Neue Exot. Dipt., p. 51, 1867. Change of name for Discocephala Macquart, 1838, not of Laporte, 1832. Type, Dasypogon abdominalis Say. Syn., Discocephala Macquart, 1838, preoceupied.

$\checkmark$ Holmbergia Arribalzaga, An. Soc. Cient. Argentina, vol. 32, p. 196, Oct., 1891. 1 species. Type, Holmbergia guntiherii Arribalzaga. Equals Rhopalosyrphus Giglio-Tos, Sept. 20, 1891.

$\checkmark$ Holoclera Schiner, Wien. Ent. Monatschr, vol. 4, p. 53, 1860. 1 species. Type, Rhamphomyia umbripennis MeIgen (as pulchra, new species). Equals Dionnæa MeIGEN, 1800.

$\checkmark$ Holoneura Kieffer, Ann. Soc. Ent. France for 1894, p. 312, 1894. 1 species. Type, Holoneura cinctu KiEFFER.

$\checkmark$ Holopogon Loew, Linn. Ent., vol. 2, p. 473, 1847. 7 species. Type, Dasypogon nigripennis Meigen, the third species, by present designation.

$\checkmark$ Holorusia Loew, Berliner Ent. Zeitschr., vol. 7, p. 277, 1863. 1 species. Type, Holorusia grandis Bergroth (as rubiginosa, new species, preoccupied). 
Homalocephala ZetTenstedt, Ins. Lapp., p. 749, 1838. 1 species. Type, Homalocephala albitarsis Zetterstedt. Syn., Psairoptera WAHLberg, 1839.

Homalomyia Bouché, Nat. Ins., p. 89, 183t. 3 species. Type, Musca canicularis Linnæus, the first species, by designation of Westwood, Intr., vol. 2, Synops. 1840, p. 143. Equals Fannia Desvoldy, 1830.

Homogenia WulP, Tijdschr. Ent., vol. 35, p. 184, 1892. 3 species. Type, Homogenia rufipes Wulp, the first species by designation of Brauer and Bergenstamm, Denkschr. Kais. Acad. Wiss. Wien, vol. 61, 1894, p. 623. Equals Trichiopoda LAtreille, 1829.

Hoplomyia Zeller, Isis von Oken for 1842, p. 882, 1842. Change of name for Stratiomys Geofrror; 1762, because the latter name was incorrectly formed. Type, Musca chamaleon Linneus. Equals Stratiomys Geoffroy, 1762.

Hormomyia Loww, Dipt. Beitr., vol. 4, p. 20, 1850. 6 species. Type, Hormomyia crassipes Loew, the second species, by present designation.

Hormopeza Zetrerstedt, Ins. Lapp., p. 540, 1838. 1 species. Type, Hormopeza obliterata ZetTerstedt.

Houghia Coqvillett, Rev. Tach., p. 118, 1897. 1 species. Type, Houghia setipennis CoqulleteT.

Howardina Theobald, Monogr. Culic., vol. 3, p. 287, 1903. 2 species. Type, Culex walkeri Thвовацd, the first species, by designation of Dyar, Proc. Ent. Soc. Washington, vol. 7, 1905, p. 49.

Hubneria Desvoldy, Ann. Soc. Ent. France for 1847, p. 601, 1847. 22 species. Type, Tachina affinis FaLLen (as Carcelia nigripes Desvoidy), the fifth species, by designation of Desvoldy, Hist. Nat. Dipt., vol. 1, 1863, p. 279. Equals Aplomya Desvoldy, 1830.

Hyadesimyia BıGot, Miss. Cape Horn, vol. 6, Zool., Dipt., p. 26, 1888. 2 species. Type, Hyadesimyia clausa Вigot, the first species, by present designation.

Hyadina Haliday, Ann. Nat. Hist., vol. 3, p. 406, 1839. 2 species. Type, Notiphila guttata FALLEN, the first species, by designation of Westwood, Intr., vol. 2, Synops., 1840, p. 1533. Equals Hydrina Desvordy, 1830.

Hyalanthrax Osten Sack EN, Biol. Centr.-Amer., Dipt., vol. 1, p. 134, 1887. 5 species. Type, Anthrax faustina Osten SACKEN, the first species, by present designation. Equals Villa Lioy, 1864.

Hyalomya Desvordy, Essai Myod., p. 298, 1830. 2 species (as 11). Type, Phasia pusilla Meigen, the last species, by designation of Westwood, Intr., vol. 2, Synops., 1840, p. 140. Equals Phasia Latreille, 1805.

Hyalomyodes Townsend, Psyche, vol. 6, p. 429, 1893. 1 species. Type, Hyalomya triangulifera Low (as weedii, new species).

Hyalurgus Brauer and Bergenstam, Denkschr. Kais. Akad. Wiss. Wien, vol. 60, p. 136, 1893. 1 species. Type, Tachina lucida Meigen.

Hybos MeigeN, Illiger's Mag., vol. 2, p. 269, 1803. No species. In his Klass. Beschr. Z weifl. Ins., 1804, pp. 240, 241, 2 species. Type, Irusca grossipes Linnæus (as funebris, new species), the first species, by designation of Curtis, Brit. Ent., 1837, p. 661. Equals Nœza MEIGen, 1800.

Hydrellia Desvorny, Essai Myod., p. 790, 1830. 11 species. Type, Notiphila flaviceps MEIGEN (as aurifacies, 'new species), the second species, by designation of Westwood, Intr., vol. 2, Synops., 1840, p. 153. Syn., Hydropota Rondanl, 1861.

Hydrina Desvordy, Essai Myod., p. 794, 1830. 7 species. Type, Notiphila guttata Fallen (as vernalis, new species), the third species, by present designation. Syn., Hyadina HaLIday, 1839.

$\checkmark$ Hydrochus Fallen, Monogr. Dolich. Sveciæ,p. 5, 1823. 4 species. Type, Hydrochus longicornis FALLEN, the first species, by present designation. Not Hydrochus Germar, 1817. Equals Perithinus Haliday, 1831. 
Hydrodromia Macevart, Hist. Nat., Dipt., vol. 2, p. 658, 1835. 2 species. Type, Heleodromia stagnalis Haldiy, the second species, by designation of Coqulllett, Proc. Ent. Soc. Washington, vol. 5, 1903, p. 251.

$\checkmark \quad$ Hydromyza Fallen, Kongl. Vet. Acad. Handl. for 1813, p. 243, 1813. 1 species. Type, Musca livens Fabricius. Syn., Nupharia Desvoidy, 1830.

$\checkmark \quad$ Hydrophoria Desvoldy, Essai Myod., p. 503, 1830. 6 species (as 9). Type, Musca vespertina FALLEN (as littoralis, new species), the last species, by designation of Westwood, Intr., vol. 2, Synops., 1840, p. 142 (as nigrita FALleN). Syn., Ilebecnema SchNabL, 1889. (Hydrophoria of authors equals Zaphne Desvoldy.)

Hydrophorus Fa LLEN, Monogr. Dolich. Sveciæ, p. 2, 1823. 7 species. Type, Hydrophorus jaculus Fallen, the last species, by designation of MACQUART, Recueil Soc. Sci. Arts Lille for 1827, p. 249. Equals Medetera Fischer, 1819. (Hydrophorus of authors equals A phrozeta Perris.)

- Hydropota Rondani, Dipt. Ital. Prodr., vol. 4, p. 10, 1861. Change of name for Iydrellia Desvoldy under the mistaken impression that it is interfered with by Hydrelia Hübner. Type, Notiphila fluviceps Melgen. Equals Hydrellia Desvoldy, 1830.

$\checkmark \quad$ Hydrotæa Desvoldy, Essai Myod., p. 509, 1830. 12 species (as 17). Type, Musca meteorica Linneus, the eleventh species, by designation of CurTis, Brit. Ent., 1839, p. 768. Syn., Lasiops Melgen, 1838; Onodontha Roxdan1, 1856; Psiloptera Lioy, 1864; Microcera Lioy, 1864.

Hygrella HaLiday, Ann. Nat. Hist., vol. 3, p. 223, 1839. 2 species. Type, Notiphila nitidula FALLEN, the first species, by designation of Westwoon, Intr., vol. 2, Synops., 1840, p. 153. Equals Psilopa Fallen, 1823.

$\checkmark \quad$ Hygroceleuthus Loew, Neue Beitr., vol. 5, p. 10, 1857. 3 species. Type, Dolichopus latipennis FaLLEN, the first species, by present designation. Equals Dolichopus Latreille, 1796.

$\checkmark$ Hygrophila Lior, Atti Inst. Veneto, ser. 3, vol. 9, p. 1102, 1864. 1 species. Type, Ephydra coarctutu FALlen? (as fascipennis MACQUART?, an error for fuscipennis). Equals Napæa Desvoiny, 1830.

Hylemya Desvoldy, Essai Myod., p. 550, 1830. 11 species (as 16). Type, Musca strigosa FABRicius (as strenua and plebeia, new species), the first species, by designation of Rondanı, Dipt. Ital. Prodr., vol. 1, 1856, p. 96. Syn., Delia Desvoldr, 1830; Egeria Desvoidy, 1830; Musciosoma Lioy, 1864; Gastrolepta Lioy, 1864; Nevrota Lioy, 1864; Pachystoma Liov, 1864.

Hylephila Rondanı, Iipt. Ital. Prodr., vol. 6, p. 13, 1877. 1 species. Type, Musca buccata Fallen. Equals Leucophora Desvoldy, 1830.

- Hyperæa Desvoldy, Hist. Nat. Dipt., vol. 2, p. 379, 1863. 1 species. Type, Hyperæa abdominalis Desvoidy. Equals Stevenia Desvoiny, 1830.

$\checkmark$ Hyperalonia Rondani, Arch. Zool., vol. 3, p. 57, 1864. 13 species. Type, Stomoxys morio FABRicius (as Anthrax erythrocephala FABricius), the fifth species, by present designation. Syn., Velocia Coquille rt, 1886.

Hyperdiplosis Felt, 23d Rep. State Ent. N. Y., p. 405, 1908. 1 species. Type, Cecidomyia lobata Feut.

$\checkmark$ Hyperecteina Schiner, Wien. Ent. Monatschr., vol. 5, p. 143, 1861. 1 species. Type, Hyperecteina metopina Schiner. Syn., Admontia Brauer and Bergenstamu, 1889.

1 Hypertrophocera Towssend, Trans. Amer. Ent. Soc., vol. 18, p. 360, 1891. 1 species. Type, Hypertrophocera parvipes Towssend. Syn., Neotractocera Townsend, 1892.

- Hyphantrophaga Townsend, Psyche, vol. 6, p. 247, 1892. 1 species. Type, Meigenia hyphantrix Townsend.

- Hypocera Lioy, Atti Inst. Veneto, ser. 3, vol. 10, p. 78, 1864. 3 species. Type, Trineura mordellaria Fallen, the first species, by designation of Brues, Gen. Ins., Dipt., Phoridæ, 1906, p. 6. Syn., Nemosia Lıoy, 1864; Gymnoptera Lıor, 1864. 
Hypochrta Brauer and Bergenstamm, Denkschr. Kais. Akad. Wiss. Wiell, vol. 56, p. 93, 1889. 1 species. Type, Tachina distincta MeIGeN (as longicornis SchIneR). Equals Campylochæta Rondani, 1859.

Hypocharassus Mıк, Verh. Zool.-Bot. Ges. Wien, vol. 28, p. 627, 1879. 1 species. Type, Hypocharassus gladiator Mik. Syn., Drepanomyia. Wheeler, 1898.

Hypoderma Latreille, Nouv. Dict. Hist. Nat., vol. 23, p. 272, 1818. 1 species. Type, Estrus bovis Linneus.

[Hypostena Meigen, Syst. Beschr. Zweifl. Ins., vol. 7, p. 239, 1838. 1 species. Type, Musca volvulus Fabricius (as procera, new species). Equals Phyllomya Desvoidy, 1830. Not American.] (Hypostena of authors equals Tachinophyto Townsend.)

Hypselura Meigen, Illiger's Mag., vol. 2, p. 273, 1803.1 species. Type, Musca fenestralis Linn wus (as senilis Fabricius). Equals Omphrale Meigen, 1800.

Hyria Desvordy, Hist. Nat. Dipt., vol. 1, p. 1100, 1863. 1 species. Type, Tachina tibialis Fallen. Not Hyria Lamarck, 1819. Equals Pelatachina Meade, 1894.

Hystrichodexia RöDen, Ent. Zeit. Stettin, vol. 47, p. 266, 1886. 1 species. Type, Hystrichodexia armata RöDER.

Hystricia Macquart, Dipt. Exot., vol. 2, pt. 3, p. 43, 1843.4 species. Type, Hystricia amœna MACQUART, the third species, by present designation.

Hystrisyphona Bigot, Rev. Mag. Zool. for 1859, p. 309, 1859. 1 species. Type, Hystrisyphona niger Bigot.

Ibisia Rondani, Dipt. Ital. Prodr., vol. 1, p. 154, 1856. 1 species. Type, Bibio marginata Fabricius. Equals Atherix Meigen, 1803.

Icaria Schiner, Reise Novara, Zool., vol. 2, Dipt., p. 276, 1868. 3 species. Type, Trypeta sparsa Wiedemann, the first species, by original designation. Not Icaria Saussure, 1853. Equals Eutreta Loew, 1873.

Ichneumonops Townsend, Smiths. Misc. Coll., vol. 51, p. 82, 1908.1 species. Type Ichneumonops mirabilis Townsend.

Icterica Loew, Monogr. Dipt. N. Amer., vol. 3, p. 287, 1873. 3 species. Type, Trypeta seriata Loew, the first species, by present designation.

Ida Desvoidy, Hist. Nat. Dipt., vol. 2, p. 389, 1863. 1 species. Type, Musca rustica FABricius (as petiolata, new species). Equals Dexia Meigen, 1826.

Idana Loew, Monogr. Dipt. N. Amer., vol. 3, p. 115, 1873.1 species. Type, Ortalis marginata $\mathrm{SAY}$.

Idioneura Philippi, Verh. Zool.-Bot. Ges. Wien, vol. 15, p. 615, 1865.1 species. Type, Idioneura macroptera PhilipPi. Equals Symplecta Meigen, 1830.

Idioplasta Osten SACKen, Cat. Dipt. N. Amer., p. 222, 1878. Change of name for Protoplasa Osten Sacken, 1860, under the erroneous impression that it interferes with Protoplasta Leidy, 1879. Type, Protoplasa fitchii Osten Sacken. Equals Protoplasa Osten SaCken, 1860.

Idiotypa Loew, Monogr. Dipt. N. Amer., vol. 3, p. 183, 1873. 1 species. Type, Idiotypa appendiculata Loew. Not Idiotypa Ferster, 1856. Equals Neoidiotypa OSTEN SACKeN, 1878.

Illigeria Desvoidy, Essai Myod., p. 273, 1830. 1 species (as 2). Type, Musca roralis Linneus. Equals Melanophora MÉIgen, 1803.

Ilythea Haliday, Ann. Nat. Hist., vol. 3, p. 408, 1839. 1 species. Type, Ephydra spilota Curtis. Syn., Epipela Stenhammer, 1844.

Imatisma Macquart, Dipt. Exot., vol. 2, pt. 2, p. 67, 1842. 2 species. Type, Eristalis posticata Fabricius, the first species, by original designation. Equals Mallota Meigen, 1822.

Iphis Meigen, Nouv. Class. Mouch., p. 27, 1800.* No species. Equals Dolichopus Latreille. Type, Musca ungulata Linnæus. Equals Dolichopus Latreille, 1796.

Ischiodonta Lioy, Atti Inst. Veneto, ser. 3, vol. 9, p. 1328, 1864. 2 species (as 3). Type, Myopa fasciata Meigen, the first species, by present designation. Equals Stomoxoides SCH AFFER, 1766. 
Ischnomyia Loew, Berliner Ent. Zeitschr, vol. 7, p. 325, 1863. 1 species. Type, Diastata? albicosta WA LKER (as vittula, new species).

- Isogasfer Lioy, Atti Inst. Veneto, ser. 3, vol. 9, p. 1114, 1864. 1 species. Type, Borborus nigrifemoratus Macquart. Equals Copromyza Fallen, 1810.

Isoglossa Coquillett, Can. Ent., vol. 27, p. 125, 1895. 1 species. Type, Isoglossa hastata Coquillett. Not Isoglossa Casey, 1893. Equals Eucoronymyia Townsend, 1908.

Isomera Desvoldy, Ann. Soc. Ent. France for 1851, p. 315, 1851. 2 species. Type, Rhedia siculu Desvoidy (as Reaumeriu blondeli I)esvoIny), the first species, hy present designation. Equals Salmacia MeIfien, 1800.

- Isopenthes Osten SAcken, Biol. Centr.-Amer., Dipt., vol. 1, p. 96, 1ss6. 2 species. Type, Isopenthes jirnnickeana Osten SACKEx, the second species, by present designation. Equals Villa Lioy, 1864.

- Isoplastus Skuse, Proc. Linn. Soc. N. S. Wales, ser. 2, vol. 4, p. 279, 1889. 3 species. Type, Isoplastus notabilis Skuse, the first species, by present designation.

- Isostomyia Coqullett, Class. Mosq., p. 16, 1906. 1 species. Type, Aëdes perturbans Wiliston.

- Istoglossa Rondani, Dipt. Ital. Prodr., vol. 1, p. 77, 1856. 1 species. Type, Istoglossa puella Rondani. Equals Clausicella Rondani, 1856.

- Itamus Loew, Linn. Ent., vol. 4, p. 84, 1849. 4 species. Type, Asilus cyanurus Loew, the first species, by present designation. Not Itamus Schмпт-Grebel, 1846. Equals Neoitamus Osten Sacken, 1878.

- Iteaphila Zettenstent, Ins. Lapp., p. 540, 1838. 2 species. Type, Iteaphila macquarti Zetterstedt, the first species, by designation of Coquillett, Proc. Ent. Soc. Washington, vol. 5, 1903, p. 251. Syn., Steleocheta BECKER, 1887.

$\checkmark$ Itonida MeIgen, Nouv. Class. Mouch., p. 19, 1800.* No species. Cecidomyia MeIgen, 1803, is a change of name. Type, Tipula pini De Geer. Syn., Cecidomyiu MeIgex, 1803; Iniplosis Loew, 1851.

$\checkmark$ Janetiella Kiefrer, Syn. Cec. Eur. Alger., p. 23, 1898. 3 species. Type, Cecidomyia thymi KIEFFER, the first species, by present designation.

J Janthinosoma Arribalzhga, Rev. Mus. La Plata, vol. 1, p. 394, 1891. 1 species. Type, Culex discrucians Walker. Syn., Conchyliastes Theobald, 1901.

$\checkmark$ Javetia Desvoldy, Hist. Nat. Dipt., vol. 1, p. 1115,1863. 1 species (as 3). Type, Tachina chalconota Meigen. Equals Macquartia Desvorny, 1830.

- Joannisia Kiefrer, Bull. Soc. Ent. France for 1894, p. 175, 1894. 2 species. Type, Jornnisia aurantiaca KiefFer, the first species, by present designation.

$\checkmark$ Joblotia Blancharn, Compt. Rend. Soc. Biol., vol. 53, p. 1046, Dec. 6, 1901 . Change of name for Trichoprosopon TнвовацD, July 15, 1901, under the mistaken idea that this is preoccupied by Trichoprosopus Macevart, 1843. Type, Trichoprosopon niripes Theobald. Equals Trichoprosopon TheoBald, July 15, 1901.

$\checkmark$ Johannseniella Willistox, Iourn. N. Y. Ent. Soc., vol. 15, p. 1, 1907. Change of name for ('ercatolophus Kieffer, 1899, not of Bocourt, 1873. Type, Ceratopogon femorata Meigen. Equals Serromyia Meigen, 1818.

Johnsonia Coquillett, Proc. Acad. Nat. Sei. Phila. for 1895, p. 316, 1895.1 species. Type, Johnsonia elegans ConvilletT.

Johnsonomyia Felt, 23d Rep. State Ent. N. Y., p. 417, 1908. 3 species. Type, Johnsonomyia rubra FELT, the last species, hy original designation.

Jurinella Brauer and Bergenstamm, Denkschr. Kais. Akad. Wiss. Wien, vol. 56, p. 132, 1889. 1 species. Type, Jurinia creruleonigra Macquart. Syn., Pseudohystricia Bracer and Bergenstama, 1889; Eujurinia Townsexd, 1908.

Jurinia Desvordy, Essai Myod., p. 34, 1830. 10 species (as 12). Type, Jurinia gagatea Desvordy, the fifth species, by present designation.

$\mathcal{J}$ Karshomyia FELT, 23d Rep. State Ent. N. Y., p. 398, 1908. 1 species. Type, Mycodiplosis viburni FeLt. 
Keratocera Desvoldy, Essai Myod., p. 788, 1830. 5 species (as 7). Type, Notiphila cinerea FALLEN (as 1 palustris, 3 trape and 4 fulvicornis, new species), by present desjignation. Equals Notiphila FAllen, 1810.

Keroplatus Bosc, Actes Soc. Hist. Nat. Paris, vol. 1, pt. 1, p. 42, 1792.1 species. Type, Keroplatus tipuloides Bosc. Syn., Cerotelion Rondani, 1856.

Kirbya Desvoiny, Essai Myod., p. 267, 1830. 1 species (as 2). Type, Tachina morens Meigen (as vernalis and hiemalis, new species). Equals khinophora DesvoIDY, 1830.

Klugia Desvordr, Hist. Nat. Dipt., vol. 1, p. 787, 1863: 1 species (as 3). Type, Tachina marginata Meigen. Equals Voria Desvordy, 1830.

Kockia Desvordy, Hist. Nat. Dipt., vol. 1, p. 818, 1863. 2 species. Type, Kockia claripennis Desvoidy, the first species, by present designation. Equals Phyto Desvolory, 1830.

Labidomyia Stephens, Syst. Cat. Brit. Ins., vol. 2, p. 239, 1829.5 species. Type, Tipula bipunctata IanNeus, the first species; by designation of Westwood, Intr., vol. 2, Synops., 1840, p. 126. Equals Culicoides Latreille, 1809.

Labigastera Macquart, Recueil Soc. Sci. Arts Lille for 1834, p. 244, 1834.2 species. Type, Tachina aurifrons Melgex (as Clelia agilis Desvoldy), the second species, by designation of Rondanı, Dipt. Ital. Prodr., vol. 1, 1856, p. 76. Equals Dionæa Desvoidy, 1830.

Laccoprosopa Townsend, Trans. Amer. Ent. Soc., vol. 18, p. 365, 1891.1 species. Type, Laccoprosopa sarcophagina Towssend. Equals Brachicoma Rondani, 1856.

Luchnomma Townsexd, Trans. Amer. Ent. Soc., vol. 19, p. 103, 1892. 1 species. Type, Atrophopoda singularis Townsend (as magnicornis, new species). Equals Paradidyma Brauer and Bergexstamu, 1891.

Lactistomyia Melander, Trans. Amer. Ent. Soc., vol. 28, p. 250, 1902.1 species. Type, Lactistomyia insolita Melander. Equals Noeza Meigen, 1800.

Lambertia Desvoidy, Hist. Nat. Dipt., vol. 2, p. 30, 1863.1 species. Type, Blondelia pallidipalpis Desvoldy. Equals Sturmia Desvordy, 1830.

Lampetia Meigen, Nouv. Class. Mouch., p. 34, 1800.* No species. Merodon MeIgen, 1803, is a change of name. Type, Syrphus clavipes Fabricius. Syn., Merodon Meigen, 1803.

Lamprempis Wheeler and Melander, Biol. Centr.-Amer., Dipt., vol. 1, p. 366, 1901. 6 species. Type, Empis chichimeca WhEeler and MELANDer, the fourth species, by designation of Coquillett, Proc. Ent. Soc. Washington, vol. 5, 1903, p. 252.

Lampria Macquart, Dipt. Exot., vol. 1, pt. 2, p 60, 1838. 2 species. Type, Laphria clavipes Fabricius, the second species, by present designation.

Laphria Meigen, Nouv. Class. Mouch., p. 25, 1800.* No species. In Illiger's Mag., vol. 2, 1803, p. 270, 6 species. Type, Asilus gibbosus Linnæus, the first species, by designation of Latreille, Consider. Général., 1810, p. 443. Syn., Ropalocera Meiger, 1820.

Laphystia Loew, Linn. Ent., vol. 2, p. 538, 1847. 1 species. Type, Laphystia sabulicola Loew.

Larvævora Meigen, Nouv. Class. Mouch., p. 38, 1800.* No species. Tachina Meigen, 1803, is a change of name. Type, Musca grossa Linnæus. Syn., Tachina Meigen, 1803; Echinomya Latreille, 1805; Faunia Đesvoidy, 1830; Cnephaliodes Brauer and Bergenstamm, 1891; Pareudora Wachtl, 1894; Nowickia Wachtl, 1894; Pararchytus Brauer and Bergenstamm, 1894; Eupeleteria Townsend, 1908.

Lasia Wiedemann, Anal. Ent., p. 11, 1824.* 1 species. Type, Lasia splendens Wiedemann.

Lasiargyra MIK, Jahresb. k. k. Akad. Gymnas., p. 5, 1878. No species. Musca diaphana FABricius, being one of the commonest species which possesses the generic characters mentioned, may be taken as the type. Type, Musca diaphana FABricius. Equals Porphyrops Meigen, 1824. 
Lasiomastix Osten Sacken, Proc. Acad. Nat. Sci. Phila. for 1859, p. 233, 1860. 1 species. Type, Limnobia macrocera SAY. Equals Phylidorea Bigot, 1854.

$\checkmark$ Lasiona Wulp, Biol. Centr.-Amer., Dipt., vol. 2, p. 127, 1890. 1 species. Type, Lasiona multisetosa WulP.

Lasioneura Coquillett, Journ. N. Y. Ent. Soc., vol. 3, p. 50, 1895. 2 species. Type, Lasioneura johnsoni Coquilletr, the first species, by present designation.

$\checkmark \quad$ Lasiophthicus Rondanı, Nuovi Ann. Sci. Nat. (Bologna) for 1844, p. 459, 1844. 1 species. Type, Musca pyrastri Linnæus. Equals Scæva Fabricius, 1805.

- Lasiopogon Loew, Linn. Ent., vol. 2, p. 508, 1847. 3 species. Type, Dasypogon pilosellus Loew (as hirtellus Meigen), the second species, by designation of Rosdani, Dipt. Ital. Prodr., vol. 1, 1856, p. 156. Syn., Daulopogon Loew, 1874.

$\checkmark$ Lasiops Melgen, Syst. Beschr. Zweifl. Ins., vol. 7, p. 323, 1838. 5 species. Type, Musca hirticeps Fallen, the fourth species, by designation of Coqulletr, Journ. N. Y. Ent. Soc., vol. 9, 1901, p. 138. Equals Hydrotæa Desvordy, 1830. (Lasiops of anthors equals Opsolasia, new name.)

$\checkmark \quad$ Lasioptera Meigex, Syst. Beschr. Zweifl. Ins., vol. 1, p. 88, 1818. 4 species. Type, Cecidomyia albipennis MElGex, the third species, by present designation. Syn., Diomyza Meigen, 1818.

$v$ Lasiosoma Winnertz, Verh. Zool.-Bot. Ges. Wien, vol. 13, p. 748, 1863.13 species. Type, Sciophila pilosa Meigen, the fourth species, by present designation. Equals Sciophila MEIGEN, 1818.

$\checkmark \quad$ Lastaurus Loew, Bemerk. Asiliden, p. 11, 1851. 2 species. Type, Dasypogon anthracinus Loww, the first species, by present designation. Syn., Morimna WaLker, 1851.

ᄂ Lathyrophthalmus Mıк, Wien. Ent. Zeit., vol, 16, p. 114, 1897. 1 species. Type, Conops æneus Scopoli. Equals Tubifera Meigen, 1800.

- Latreillia Desvoiny, Essai Myod., p. 104, 1830. 5 species (as 10). Type, Musca bifasciata Fabricius, the first species, by present designation. Not Latreillia Roux, 1827. Equals Belvosia Desvordy, 1830.

$\checkmark$ Latreillimyia Townsend, Smiths. Misc. Coll., vol. 51, p. 105, 1908. Change of name for Latreillia Desvordy, 1830, not of Roux, 1827. Type, Musca bifasciata Fabricius. Equals Belvosia Desvordy, 1830.

$\checkmark$ Lauxania Latreille, Hist. Nat. Crust. et Ins., vol. 14, p. 390, 1805, 1 species. Type, Musca cylindricornis Fabricius.

Laveramia Theoвald, Journ. Trop. Med., vol. 5, p. 183, June 16, 1902.1 species. Type, Anopheles argyritarsis Desvoiny. Not Laverania Grassi and Felettr, 1890. Equals Cellia Th вовацd, June 16, 1902.

- Leia Meigex, Syst. Beschr. Zweifl. Ins., vol. 1, p. 253, 1818. 10 species. Type, Leia fascipennis Meigen, the fifth species, by designation of Curtis, Brit. Ent., 1837, p. 645. Syn., Lejomya Rondani, 1856; Lejosoma Rondanı, 1856; Glaphyroptera Winnertz, 1863; Neoglaphyroptera Osten SACK EN, 1878. (Leia of authors equals Rondaniella, Johannsen.)

$\checkmark$ Lejogaster Rondani, Dipt. Ital. Prodr., vol. 2, p. 166, 1857. 1 species. Type, Chrysogaster splendida Meigen (as tarsatus Meigen). Equals Chrysogaster Meigen, 1800.

- Lejomya Rondanı, Dipt. Ital. Prodr., vol. 1, p. 195, 1856. 1 species. Type, Mycetophila bimaculata Meigen. Equals Leia Meigen, 1818.

Lejops Rondani, Dipt. Ital. Prodr., vol. 2, p. 33, 1857. 1 species. Type, Mallota vittata Meigen. Equals Helophilus Meigen, 1822.

$\checkmark$ Lejosoma Rondaxi, Dipt. Ital. Prodr., vol. 1, errata, 1856. Çhange of name of Lejomya Rondani, 1856. Type, Mycetophila bimaculata Meigen. Equals Leia Meigen, 1818.

Lemtopeza Macquart, Ins. Dipt. Nord France, Separata, p. 143, 1827. 1 species. Type, Ocydromia flavipes MeIg Ex (as flavipes, new species). 
Lentiphora Desvoidy, Essai Myod., p. 656, 1830. 1 species. Type, Lentiphora flaveola Desvoidy. Syn., Tephrochlamys Loew, 1862.

Lepidanthrax Osten SACKen, Biol, Centr.-Amer., Dipt., vol 1, p. 107, 1886. 3 species. Type, Anthrax disjuncta Wiedemann, the first species, by present designation.

Lepidomya Bigot, Ann. Soc. Ent. France for 1857, p. 557, 1857. 1 species. Type, Empis melanocephala Fabricius (as Hemerodromia mantispa MeIgev). Equals Hemerodromia MeIgen, 1822.

Lepidomyia LoEw, Berliner Ent. Zeitschr., vol. 8, p. 69, 1864. 1 species. Type, Lepidomyia calopus Loew. Syn., Lepromyia Williston, 1886; Lepidostola Mıк, 1886.

Lepidophora Westwood, Lond. Edinb. Philos. Mag., vol.6, p. 447, 1834.1 species. Type, Ploas ægeriiformis GraY.

Lepidoplatys Coquillett, Science, vol. 23, p. 314, 1906. 1 species. Type, Culex squamiger CoQuilletT.

Lepidosia Coquillett, Science, vol. 23, p. 314, 1906. 1 species. Type, Culex cyanescens Coquillett.

Lepidostola Mıк; Wien. Ent. Zeit., vol. 5, p. 278, Oct., .1886. Change of name for Lepidomyia LoEw, 1864, under the mistaken impression that it is preoccupied by Lepidomya Bıgoт, 1857. Type, Lepidomyia calopus Loew. Equals Lepidomyia LoEw, 1864.

Lepiselaga Macquart, Dipt. Exot., vol. 1, pt. 1, p. 153, 1838. 1 species. Type, Himatopota crassipes Fabricius (as Tabanus lepidota Wiedemann). Equals Hadrus PeRTY, 1834.

Lepopteryx Speiser, Arch. Natur. for 1900, p. 53, 1900. 2 species. Type, Lipoptena phyllostomatis PERTy, the first species, by original designation. Equals Aspidoptera Coquillett, 1899.

Lepromyia Williston, Synops. N. Amer. Syrph., p. 31, 1886. Change of name for Lepidomyia Loww, under the mistaken impression that the latter is preoceupied by Lepidomya Bigot. Type, Lepidomyia calopus LoEw. Equals Lepidomyia LoEw, 1864.

Leptina Meigen, Syst. Beschr. Zweifl. Ins., vol.6, pl. 65, fig 10, 1830.1 species. Type, Tipula albipes Sтroм (as Dolichopeza sylvicola CurTIs). Equals Dolichopeza CurTis, 1825.

Leptis Fabricius, Syst. Antliatorum, p. 69, 1805. Change of name of Rhagi FABRICIUS, 1775, under the mistaken impression that it is preoccupied by Rhagium $\mathrm{F}_{\mathrm{A}-}$ BRIcius, 1775, Type, Musca scolopacea Linneus. Equals Rhagio Fabricius, 1775.

Leptocera Olivier, Mem. Soc. Agric. Dep. Seine, vol. 16, p. 16, 1813.* 1 species. Type, Leptocera nigra Olivier. Syn., Nerea Desvoidy, 1830; Bacchis Desvoidy, 1830; Limosina Macquart, 1835; Gonioneura Rondani, 1880; Elachisoma Rondani, 1880.

Leptochilus Loew, Berliner Ent. Zeitschr., vol.16, p. 254, 1872. 1 species. Type, Leptochilus modestus Loew. Not Leptochilus SAUssure, 1852. Equals Epacmus Osten SACken, 1887.

Leptocorypha ALDRICH, Trans. Ent. Soc. Lond. for 1896, p. 315, 1896. 1 species. Type, Leptocorypha pavo Aldrich.

Leptoda Wulp, Tijdschr. Ent., vol. 28, p. 196, 1885. 2 species. Type, Dexia gracilis Wiedemann, the first species, by designation of Wulp, Biol. Centr.-Amer., Dipt., vol. 2, 1891, p. 250.

Leptodiplosis KIEFFer, Bull. Soc. Ent. France for 1894, p. 28, 1894. 5 manuscript species. Type, Leptodiplosis septemguttata KiefFer (equals Cecidomyia pictipennis Perris, 1870, not of Meigen, 1830), the second species, by designation of Kieffer, Bull. Soc. Ent. France, 1894, page cclxxx. Syn., Coprodiplosis Kiefrer, 1894.

Leptogaster Meigen, Illiger's Mag., vol. 2, p. 269, 1803.1 species. Type, Asilus cylindricus De Geer (as tipuloides Fabricius). Syn., Gonypes Latreille, 1805. 
$\checkmark$ Leptomorphus CurTis, Brit. Ins., p. 365, 1831. 1 species. Type, Leptomorphus walkeri CurTis.

- Lepiomydas Gerstecker, Ent. Zeit. Stettin, vol. 29, p. 81, 1868. 8 species. Type, Mydus lusitanicus Wiedemann, the first species, by present designation.

$\checkmark$ Leptomyza Macquart, Hist. Nat., Dipt., vol. 2, p. 580, 1835. Change of name for Anthomyzu Fallen, 1810, supposed to interfere with Anthomyia Meigen, 1803. Type, Anthomyza gracilis Fatlen. Equa!s Anthomyza Falden, 1810.

$\checkmark$ Leptophora Six, Tijdschr. Ent., vol. 21, p. 185, 1878. 1 species. Type, Phora galeata Haliday (as perpusilla, new species). Equals Metopina Macquart, 1835.

$\checkmark$ Leptopus Fallen, Monogr. Dolich. Sveciæe, p. 23, 1823. 4 species. Type, Dolichopus cortristans Wiedenann, the third species, by present designation. Not Leptopus Latreille, 1809. Equals Sciapus Zeller, 1842.

$\checkmark$ Leptopus Halidax, Zool. Journ., vol. 5, p. 358, 1831. 2 species. Type, Medetera ornata HALIDAY, the second species, by present designation. Not Leptopus Latreille, 1809. Equals Xanthochlorus LoEw, 1857.

$\checkmark$ Leptorhethum Aldrici, Kansas Univ. Quart., vol. 2, p. 50, 1893. 1 species. Type, Leptorhethum angustatum ALDRicH.

- Leptorhina Stephens, Syst. Cat. Brit. Ins., vol. 2, p. 243, 1829. 1 species. Type, Limnobia longirostris Wiedemann. Equals Megarhina St. Fargeau and Serville, 1828.

Leptosceles Halidar, Ent. Mag., vol. 1, p. 160, 1833. 3 species. Type, Leptosceles guttata Haliday, the first species, by designation of Coquillett, Proc. Ent. Soc. Washington, vol. 5, 1903, p. 252. Equals Dolichocephala Macquart, 1823.

- Leria Desvondy, Essai Myod., p. 653, 1830. 7 species. Type, Musca serrata LiNNEUS (as fenestrarum, new species), the fourth species, by designation of RoNDAN1, Dipt. Ital. Prodr., vol. 1, 1856, p. 103. Equals Heleomyza FALlex, 1810.

- Leskia Dessordy, Essai Myod., p. 100, 1830. 1 species. Type, Tachina aurea Fallen (as flarescens, new species). Syn., Myobiu Desvoldy, 1830; Hebia Desvoldy, 1830; Solieria Desvondy, 1848; Orellia Desvoidy, 1848; Pyrrosia Rondani, 1856; Anthoica Rondani, 1861; Chremia Desvoldy, 1863.

- Leskiomima Brauer and Bergenstamm, Denkschr. Kais. Akad. Wiss. Wien, vol. 58 , p. 372, 1891 . 1 species. Type, Stomoxys tenera Wiedemann.

- Lesticocampa DYAR and KNab, Journ. N. Y. Ent. Soc., vol. 14, p. 226, 1906.1 species. Type, Wyeomyia lunata TheовацD.

- Lestomyia Williston, Trans. Amer. Ent. Soc., vol. 11, p. 19, 1883. 2 species. Type, Clavator salulonum Osten SACKen, the first species, by original designation.

$\checkmark$ Lestophonus Williston, Ins. Life, vol. 1, p. 21, 1888. 1 species. Type, Lestophonus iceryie Williston. Equals Cryptochetum Rondani, 1875.

- Lestremia Macquart, Rec. Trav. Soc. Sci. Lille for 1826, p. 173, 1826. 1 species. Type, Lestremia cinerea Macquart. Syn., Mimosciara Rondani; 1840; Cecidogona Loew, 1844; Furcinerva Rondani, 1846; Yposatcea Rondanl, 1856.

$\checkmark$ Leucomelina Macquart, Dipt. Exot., Suppl. 4, p. 261, 1851. 1 species. Type, Leucomelina pica MACQUART.

- Leucophenga $\mathrm{M}_{1 \mathrm{~K}}$, Wien. Ent. Zeit., vol. 5, p. 317, 1886. 1 species. Type, Drosophela maculata Dufour.

$\checkmark$ Leucophora Desvoldy, Essai Myod., p. 562, 1830. 5 species. Type, Leucophora cinerea Desvoldy, the third species, by designation of CoqullLetT, Journ. N. Y. Ent. Soc., vol. 9, 1901, p. 138. Syn., Ocromyia Lior, 1864; Hylephila Rondani, 1877; Hammomyia RoNDANI, 1877.

$\checkmark$ Leucopis Meigex, Syst. Beschr. Zweifl. Ins., vol. 6, p. 133, 1830. 4 species. Type, Leucopis annulipes ZeTterstent (as griseola MEIGeN), the first species, by designation of Blanchard, Hist. Nat. Ins., vol. 3, 1840, p. 627.

$\mathcal{L}$ Leucostola Loew, Neue Beitr., vol. 5, p. 39, 1857. 1 species. Type, Dolichopus vestita WIEDEMANN. 
Leucostoma Meigen, Illiger's Mag., vol. 2, p. 279, 1803. No species. In his Syst. Beschr., vol. 4, 1824, p. 234, 1 species. Type, Ocyptera simplex Fallen. Syn., Clelia Desioidy, 1830; Psalida Rondani, 1856; Calyptidia Desvoidy, 1863.

Leucozona Sch rner, Wien. Ent. Monatschr., vol. 4, p. 214, 1860. 1 species. Type, Musca lucorum Linneus. Equals Syrphus Fabricius, 1775.

Liancalus Loew, Neue Beitr., vol. 5, p. 22, 1857. 2 species. Type, Musca virens Scopoll, the first species, by present designation. Equals Orthoceratium Schrank, 1803.

Lilæa WAlker, Ins. Saund., Dipt., p. 11, 1850. 2 species. Type, Pangonius luridus W W Alker, the second species, by present designation. Equals Pangonius Latreille, 1802.

Limatus Theobald, Monogr. Culic., vol. 2, p. 349, 1901. 1 species. Type, Iimatus durhamii Theobald. Syn., Simondella Laveran, 1902.

Limnobia Meigen, Syst. Beschr. Zweifl. Ins., vol. 1, p. 116, 1818. Change of name for Limonia Meigen, 1803, because the latter name has anothor meaning beside that intended. Type, Tipula tripunctata Fabricius. Equals Amphinome Meigen, 1800.

Limnobiorhynchus Westwood, Ann. Soc. Ent. France for 1835, p. 683, 1835. 2 species. Type, Limnobiorhynchus brasiliensis Westwood, the first species, by present designation. Equals Geranomyia Halidar, 1833.

Limnoea Rondanı, Dipt. Ital. Prodr., vol. 1, p. 181, 1856.1 species. Type, Tipula flavescens Linnæus. Equals Polymeda Meigen, 1800.

Limnoicr Rondan1, Dipt. Ital. Prodr., vol. 4, p. 11, 1861. Change of name for Limnoa Rondani, 1856, on account of Limnica PoLI, 1791. Type, Tipula flarescens Linneus. Equals Polymeda Meigen, 1800.

Limnomyza Rondan1, Dipt. Ital. Prodr., vol. 1. 1. 185, 1856. 1 species. Type, Tipula tripunctata Fabricius. Equals Amphin -

[Limnophila Macquart, Hist. Nat., J:..., vol. 1, p. 95, 1834. 21 species. Type, Limnobia pictipennis Mergex, the first species, by designation of Westwoon, Intr., vol. 2, Synops., 1840, p. 128. Syn., Limnomya Rondani, 1861; Pocilostola Schiner, 1863. Not American.] (Limnophila of authors equals Phylidorea Bıсот.)

Limnophora Desvolny, Essai Myod., p. 517, 1830. 20 species. Type, Limnophora palustris Desvoidy, the second species, by present designation. Syn., Gymnodia Desvoidy, 1863; Melanochelia Rondani, 1866; Brontra Kowarz, 1873; Pseudolimnophora Strobl, 1893; Stroblia Pokorny, 1893; Neolimnophora Schnabl, 1902.

Limnospila Schnabl, Wien. Ent. Zeit., vol. 21, p. 111, 1902. 1 species. Type, Aricia albifrons Zetterstedt. Equals Cœnosia Meigen, 1830.

Limonia MeIgen, Illiger's Mag., vol. 2, p. 262, 1803. 5 species. Type, Tipula tripunctata Fabricius, the first species, by designation of Westwoon, Intr., vol. 2, Synops., 1840, p. 129 . Equals Amphinome Meigen, 1800.

Limosia Desvordy, Essai Myod., p. 535, 1830. 14 species (as 28). Type, Limosia campestris Desvoidy, the sixth species, by designation of Coquillett, Journ. N. Y. Ent. Soc., vol. 9, 1901, p. 138. Equals Cœnosia Meigen, 1830.

Limosina Macquart, Hist. Nat., Dipt., vol. 2, p. 571, 1835.9 species. Type, Borborus sylraticus MeIgen, the first species, by designation of Westwood, Intr., vol. 2, Synops., 1840, p. 145. Equals Leptocera Olivier, 1813.

Linnæmya Desvoldy, Essai Myod., p. 52, 1830. 3 species (as 7). Type, Tachina vulpina FALLEN (as silvestris, new species), the second species, by designation of Desvoidy, Hist. Nat. Dipt., vol. 1, 1863, p. 131. Syn., Bonellia Desvordy, 1830.

Liogma Osten Sacken, Monogr. Dipt. N. Amer., vol. 4, p. 298, 1869.2 species. Type, Triogma nodicornis Osten Sacken, the second species, by present designation.

Lipochæta Coquilletr, Ent. News, vol. 7, p. 220, 1896. 1 species. Type, Lipochæta slossonæ Coquillett.

Proc.N.M.vol.38-10-36 
Lipoptena Niтzsch, Germ. Mag. Ent., vol. 3, p. 310, 1818. 1 species. Type, Pediculus cervi Linneus (as cervina Panzer). Syn., Hrmobora Curtis, 1824; Ornithobia Meigen, 1830; Alcephagus Gimerthal, 1845.

$\checkmark$ Liriope Meigen, Nouv. Class. Mouch., p. 14, 1800.* No species. Ptychoptera Meigen, 1803, is a change of name. Type, Tipula contaminata Linneus. Syn., Ptychoptera Meigen, 1803; Ctenoceria Rondani, 1856.

- Lisella Desvoidy, Essai Myod., p. 649, 1830. 1 species. Type, Musca flava Linnkus (as flara, new species). Equals Chyromya Desvoidy, 1830.

- Lispe Latreille, Precis, p. 169, 1796. No species. In 1802, 1 species. Type, Musca tentaculata DE GEER.

$\checkmark$ Lispidea Coquillett, Journ. N. Y. Ent. Soc., vol. 3, p. 51, 1895.1 species. Type, Lispidea palpiger CǫUilletT.

$\checkmark$ Lissa Melgen, Syst. Beschr. Zweifl. Ins., vol. 5, p. 370, 1826. 1 species. Type, Ocyptera dolium Fabricius. Not Lissa Leach, 1815. Equals Megamerina Rondani, 1861.

$\checkmark$ Listeria Desvordy, Hist. Nat. Dipt., vol. 2, p. 600, 1863. 1 species. Type, Mrusca lineata Fallen (as agrestis, new species). Equals Sarcophaga Meigen, 1826.

$\checkmark$ Listropoda Kolenat, Wien. Ent. Monatschr., vol.1, p. 62, 1857. 4 species. Type, Listropoda blasii Kolenati, the second species, by present designation. Equals Nycteribia LATreille, 1796.

$\checkmark$ Litanomyia Melander, Trans. Amer. Ent. Soe., vol. 28, p. 231, 1902. 2 species. Type, Sciodromia mexicana Wheeler and Melander, the first species, by designation of Coquillett, Proc. Ent. Soc. Wash., vol.-5, 1903, p. 252. Equals Chyromantis RONDANI, 1856.

Litorella Rondani, Dipt. Ital. Prodr., vol. 1, p. 101, 1856. 1 species. Type, Ochtiphila litorella FaLLen. Equals Schœenomyza. HaLiday, 1833.

ᄂ Litorhynchus MAcquart, Iipt. Exot., vol. 2, pt. 1, p. 78, 1840. 4 species. Type, Litorhynchus hamatus MACQUART, the second species, by present designation. Equals Mima MeIGen, 1820.

- Lixophaga Townsend, Smiths. Misc. Coll., vol. 51, p. 86, 1908. 1 species. Type, Lixophage parva Townsend. Equals Tachinophyto Townsend, 1892.

- Lobodiplosis FeLt, 23d Rep. State Ent. N. Y., p. 397, 1908. 2 species. Type, Micodiplosis acerina FELT, the first species, by original designation.

Lobopteromyia Felt, 23d Rep. State Ent. N. Y., p. 389, 1908. 8 species. Type, Cecidomyici filicis FELT, the first species, by original designation.

$\checkmark$ Loewiella Williston, Man. N. Amer. Dipt., p. 57, 1896. Change of name for Blacodes Loew, 1874, not of Dejean, 1859. Type, Blax belhus Loew. Equals Cophura Osten SACKEN, 1887.

$\checkmark$ Lonchæa FAllen, Ortalides Sveciæ, p. 25, 1820. 3 species. Type, Musca chorea Fabricius, the second species (as vaginalis, new species), by designation of Westwoon, Intr., vol. 2, Synops., 1840, p. 150. Syn., Teremyia MIACQUART, 1835; Earomyia Zetterstedt, 1842; Dasiops Rondani, 1856.

$\checkmark$ Lonchopalpus Desvordy, Dipt. Env. Paris, Myop., p. 35, 1853.* 1 species. Type, Myopa dorsalis Fabricius. Equals Stomoxoides Schaeffer, 1766.

Lonchoptera Meigen, Illiger's Mag., vol. 2, p. 272, 1803. No species. In 1824, 10 supposed species. Type, Lonchoptera lutea PANzer, the first species, by designation of Cuntis, Brit. Ent., 1839, p. 761. Syn., Dipsa Fallen, 1810.

Longurio Loew, Berliner Ent. Zeitschr., vol. 13, p. 3, 1869. 1 species. Type, Longurio testaceus LoEw.

$\checkmark \quad$ Lophonotus Macquart, Dipt. Exot., vol. 1, pt. 2, p. 125, 1838. 12 species. Type, Asihus chalcogaster WIEDEMANN (as auribarbis, new species), the first species, by present designation. Not Lophonotus Stephens, 1829. Equals Dysmachus Loew, 1860.

Lophoteles LoEw, Berliner Ent. Zeitschr., vol. 2, p. 110, 1858. 1 species. Type, Lophoteles plumula LoEw 
Lordatia Desvordy, Essai Myod., p. 808, 1830. 2 species (as 5). Type, Lordatia coprina Desvordy, the second (fourth supposed) species, by present designation. Equals Cypsela Meigen, 1800.

Lordotus Loww, Berliner Ent. Zeitschr., vol. 7, p. 303, 1863. 1 species. Type, Lordotus gibbus LoEw.

Lotobia Lrox, Atti Inst. Veneto, ser. 3, vol. 9, p. 1114, 1864. 1 species. Type, Borborus pallidicentris Meigen. Equals Cypsela Meigen, 1800.

toxocera Meigen, Illiger's Mag., vol. 2, p. 275, 1803. 1 species (as 2). Type, Musca ichneumonea Linnæus.

Lucilia Desvoidy, Essai Myod., p. 452, 1830. 17 species (as 37). Type, Musca cxsar LinNxus, the first species, by designation of Macquart, Recueil Soc. Sci. Arts Lille for 1833-34, p. 162. Syn., Mya Rondani, 1850; Somomya Rondani, 1861; Phenicia Desvorny, 1863.

Lutzia Theoвald, Monogr. Culic., vol. 3, p. 155, 1903. 1 species. Type, Culex bigotii BELLARDi.

Lycastrirhyncha BIGot, Rev. Mag. Zool. for 1859, p. 3, 1859. 1 species. Type, Lycastrirhyncha nitens Вı̈от.

Lycia Desvoldy, Essai Myod., p. 637, 1830. 10 species. Type, Sapromyza rorida FALLEN (as flava, new species), the first species, by present designation. Equals Sapromyza FALLEN, 1810.

Lycoria Meigen, Nouv. Class. Mouch., p. 17, 1800.* No species. Sciara Meigen, 1803, is a change of name. Type, Tipula thomæ Linneus. Syn., Sciara Meigen, 1803; Molobrus Latreille, 1805.

Lydella Desvoiny, Essai Myod., p. 112, 1830. 7 species (as 12). Type, Tachina nigripes FALLEN (as grisescens, new species), the first species, by designation of Desvordy, Hist. Nat. Dipt., vol. 1, 1863, p. 855. Syn., Blondelia Desvoidy, 1830; Anetia Desvoiny, 1863; Cyrillia Desvoldy, 1863; Picconia Desvorny, 1863; Gerraisia Desvoiny, 1863; Dexodes Brauer and Bergenstamm, 1889; Aporoluchina Meade, 1894; Allophorocera Hendel, 1901; Paradegodes Townsend, 1908.

Lydina Desvoldy, Essai Myod., p. 124, 1830. 1 species (as 3). Type, Tachina ænea Meigen. Syn., Harrisia Meigen, 1838; Polidea Macquart, 1848; Somoleja Rondani, 1865.

Lynchia Weyenberg, Anal. Soc. Cient. Argentiph. for 1881, p. 195, 1881. 1 species. Type, Lynchia penelopes Weyenberg.

Lynchiella Lahille, Act. Trab. Congr. Med. Lat.-Amer., vol. 2, p. 13, 1904. Change of name of Megarhinus Desvoidy, 1827, under the mistaken idea that the latter is preoccupied by Megarhina St. Fargeau and Serville, 1828. Type, Culex hrmorrhoidalis Fabricius. Equals Megarhinus Desvoidy, 1827.

Lyperosia Rondanı, Dipt. Ital. Prodr., vol. 1, p. 93, 1856. 1 species. Type, Conops irritans Linneus. Equals Hæma:obia St. Fargeau and Serville, 1828.

Lyphe Desvoidy, Essai Myod., p. 141, 1830. 1 species (as 2). Type, Tachina dubia Fallen. Syn., Aporomya Rondni, 1859; Enthenis Desvoidy, 1863.

Lyroneurus Loew, Wien. Ent. Míonatschr., vol. 1, p. 38, 1857. 2 species. Type, Lyroneurus cœrulescens LoEw, the first species, by present designation. Equals Diaphorus Meigen, 1824.

Lythia Desvoldy, Hist. Nat. Dipt., vol. 1, p. 707, 1863. 1 species. Type, Lythia flavicornis Desvoidy. Equals Ceranthia Desvoidy, 1830.

Lytogaster Bескек, Berliner Lnt. Zeitschr., vol. 41, p. 202, 1896. 1 species. Type, Philygria abdominalis Stenhammer.

V Macellocerus Mıк, Jahresb. k.-k. Akad. Gymas., p. 3, 1878. 1 species. Type, Tachytrechus mœchus LoEw. Equals Tachytrechus HaLIDAy, 1851.

Machrræa Rondani, Dipt. Ital. Prodr., vol. 3, p. 159, 1859. 1 species. Type, Tachina concinnata Meigen (as serriventris, new species). Equals Compsilura Bouché, 1834. 
Machimus Loew, Linn. Ent., vol. 4, p. 1, 1849. 15 species. Type, Asilus chrysitis MeIGEN, the second species, by present designation.

Macquartia Desvoldr, Essai Myod., p. 204, 1830. 5 species (as 8). Type, Tachina chalconota MEIGEN (as germanica and viridescens, new species), the fourth and fifth supposed species, by designation of Rondani, Dipt. Ital. Prodr., vol. 1, 1856, p. 65. Syn., Amedea Desvoidy, 1830; Albinia Desvoidy, 1830; Gymnopsis Rondavi, 1859; Cleonice Desvond, 1863; Bebriria Desvonny, 1863; Javetiu Desvoldy, 1863; Pherecida Desvoldy, 1863; Rondanimyia Townsend, 1908.

- Marrocera M EIG en, Illiger's Mag., vol. 2, p. 261, 1803. No species. In his Klass. Beschr. Zweifl. Ins., 1804, pp. 46, 47, 3 species. Type, Macrocera luten MEIgen, the first species, by designation of CurTis, Brit. Ent., 1837, p. 637. Equals Euphrosyne Meigen, 1800.

- Macroceromys Bigot, Bull.Soc. Ent. France for 1877, p. 73, 1877. 1 species. Type, Macroceromys fulvirentris Bigot. Equals Solva WALKER, 1860.

- Macrochetum Rondani, Dipt. Ital. Prodr., vol. 1, p. 127, 1856. 1 species. Type, Oscinis cornuta Fallen. Equals Crassiseta von Roser, 1840.

- Macrochira Meigen, Illiger's Mag., vol. 2, p. 278, 1803. 1 species. Type, Musca mantis De Geer (as manicata Fabricius). Equals Ochthera Latreille, 1802.

- Mucrochira ZetTerstedt, Ins. Lapp., p. 784, 1838. 1 species. Type, Heteromyza fava Meigen (as flava, new species). Not Machrochira Meigen, 1803. Equals Clusia HALIDAY, 1838.

Macromeigenia Brauer and Bergenstamm, Denkschr. Kais. Akarl. Wiss. Wien, vol. 58, p. $311 ; 1891.1$ species. Type, Tachina chrysoproctu Wiedemann.

Macrometopa Brauer and Bergenstami, Denkschr. Kais. Akar. Wiss. Wien, vol. 56, p. 117, 1889 . 1 species. Type, Microphthalma calogaster Biciot (as mexicana, new species).

$\checkmark$ Macronevra Macquart, Hist. Nat., Dipt., vol. 1, p. 146, 1834. 1 species. Type, Mycetobia ferruginosa Meirien (as winthemi, new species). Equals Diadocidia Ruthe, 1831.

$\checkmark$ Mucronichic Rondani, Dipt. Ital. Prodr., vol. 3, p. 229, 1859. 1 species. Type, Millogramme ungulans PANDELle (as Tachina agrestis FAlLex). Equals-Amobia Desvoldy, 1830.

$\checkmark$ Macronix Bigot, Ann. Soc. Ent. France for 1857, p. 549, 1857. 1 species. Type, Dasypogon lomginngulatus MACQUart. Equals Dicranus Loew, 1851.

Macroptera Lioy, Atti Inst. Veneto, ser. 3, vol. 9, p. 224, 1864. 1 species. Type, Limnobia macroptera Macquart. Equals Ula Haliday, 1833.

2 Macrorrhyncha Winnertz, Ent. Zeit. Stettin, vol. 7, p. 16, 1846. 1 species. Type, Macrorrhyncha flava Winnertz. Equals Asindulum Latreille, 1805.

- Macrosargus Bıgot, Ann. Soc. Ent. France for 1879, p. 187, 1879. Change of name of Pedicella Bigot, 1856. Type, Macrosargus tenuiventris Bıяот. Equals Pedicella Bigot, 1856.

$\checkmark$ Macrostenomyia Hendel, Wien. Ent. Zeit., vol. 26, p. 98, 1907. Change of name for Stenomacra Loew, 1873, not of Stal, 1870. Type, Sepsis guerini Bigot. Syn., Stenomacra LoEw, 1873, preoccupied.

$\checkmark$ Macrostomus Wiedemann, Zool. Mag., vol. 1, pt. 1, p. 60, 1817. 1 species. Type, Hylos ferrugineus Fabricius. Equals Dionnæa Meigen, 1800.

$\checkmark \quad$ Macrostyla Liox, Atti Inst. Veneto, ser. 3, vol. 9, p. 1126, 1864. 1 species. Type, Chlorops plumiger Meigen. Equals Botanobia Lıoy, 1864.

$\checkmark$ Macrurus Lioy, Atti Inst. Veneto, ser. 3, vol. 9, p. 1313, 1864. 1 species. Type, Agromyza latipes Meigen. Not Macrumes Bonaparte, 1841. Equals Desmometopa LoEw, 1865.

$\checkmark$ Madiza FAllen, Spec. Ent. Meth. Exh., p. 19, 1810. No species. In 1820, 5 species. Type, Madiza uscinina Fallen, the second species, by designation of Rondani, Dipt. 
Ital. Prodr., vol. 1, p. 128, 1856. Syn., Siphonella Macquart, 1835; Siphunculina Rondani, 1856; Eurinella Meunier, 1893.

Mækistocera Wiedemann, Dipt. Exot., p. 41, 1821. 2 species. Type, Tipula filipes Fabricius, the second species, by designation of Macquart, Dipt. Exot., vol. 1, pt. 1,1838 , p. 59.

Mallophora Macquart, Hist. Nat., Dipt., vol. 1, p. 300, 1834. 5 species. Type, Asilus bomboides Wiedemann, the second species, by present designation.

Mallota Meigen, Syst. Beschr. Zweifl. Ins., vol. 3, p. 377, 1822. 3 species. Type, Syrphus fuciformis Fabricius, the third species, by designation of Rondani, Nuovi Ann. Sci. Nat. (Bologna), 1844, p. 452. Syn., Imatisma MACQuart, 1842; Zetterstertia Rondani, 1844.

Mancia Coqulletet, Can. Ent., vol. 18, p. 159, 1886. 1 species. Type, Mancia nana Coquillett.

Manota Williston, Trans. Ent. Soc. Lond. for 1896, p. 260, 1896.1 species. Type Manota defecta Williston.

Mansonia Blanchard, Comp. Rend. Soc. Biol. for 1901, p. 1046, 1901. Change of name for Panoplites Theobald, 1900, not of Govld, 1853. Type, Culex titillans Walker. Equals Tæniorhynchus A.rRibalzaga, 1891.

- Mantipeza Rondani, Dipt. Ital. Prodr., vol. 1, p. 148, 1856.1 species. Type, Tachydromia prxcatoria FALLEN (as Hemerodromia monostigma MeIgen). Equals Chelifera Macquart, 1823.

- Maravigna Lioy, Atti Inst. Veneto, ser. 3, vol. 9, p. 891, 1864. 1 species. Type, Tachina obsoleta Meigen (as clausa Macquart). Equals Onesia Desvoidy, 1830.

Marginomyia Meigen, Syst. Beschr. Zweifl. Ins., vol. 1, p. 147, 1818. 1 species. Type, Limnobia cinerascens Meigen. Syn., Dicranoptycha Osten Sack en, 1860.

Marshamia Desvoidy, Essai Myod., p. 57, 1830. 1 species (as 2). Type, Tachina comta Fallen. Equals Bonnetia Desvoidy, 1830.

- Masicera Macquart, Rec. Trav. Soc. Sci. Lille for 1834, p. 285, 1834.10 species.

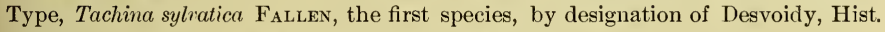
Nat. Dipt., 1863, p. 872 (as Phryxe paronix Desvoldy).

Masiphya Braver and Bergenstamm, Denkschr. Kais. Akad. Wiss. Wien, vol. 58, p. 313, 1891. 1 species. Type, Masiphya brasiliana Brauer and Bergenstamm. Equals Biomya RoNdani, 1856.

Masipoda Brauerand Bergenstamm, Denkschr. Kais. Akad. Wiss. Wien, vol. 56, p. 162, 1889. 1 species. Type Exorista rufilatera Rondani (as geminata, new species). Equals Winthemia Desvoldy, 1830.

- Masistylum Brauer and Bergenstama, Denkschr. Kais. Akad. Wiss. Wien, vol. 60, p. 125. 1893. 1 species. Type, Pachystylum arcuatum Miк.

Mauromyia Coquilletr, Rev. Tach., p. 51, 1897. 1 species. Type, Mauromyia pulla Coqulletet.

Mayetia Kieffer, Misc. Ent., vol. 4, p. 5, 1896. 1 species. Type, Cecidomyia destructor Say. Equals Phytophaga Rondani, 1840.

Meckelia Desvoidy, Essai Myod., p. 714, 1830. 2 species. Type, Musca hortulana Rossi (as Oscinis elegans Desvoldr), the first species, by present designation. Not Meckelia Leuckart, 1828. Equals Ceroxys Macquart, 1835.

Medetera Fischer, Programme Not. Mouche Carn., p. 11, 1819.* 1 species. Type, Musca diadema Linneus (as carnivorus, new species). Syn., Hydrophorus Fallen, 1823; Trchobates Haliday, 1831; Orthobates WahlberG, 1844; Anort'lus LoEw, 1850; Oligochrtus Mik, 1878.

Medina Desvoidy, Essai Myod., p. 138, 1830. 4 species (as 6). Type, Tachina collaris FALLEN (as cylindrica, new species), the second species, by present designation. Syn., Degeeria Meigen, 1838; Amedoria Brauer and Bergenstamm, 1889. 
Megacyttarus Bigot, Bull. Soc. Ent. France for 1880 , p. 47, 1880. 1 species. Type, Rhamphomyia limbata Loew (as argenteus, new species). Equals Dionnæa Meigen, 1800.

Megaglossa Rondan, Bull. Soc. Ent. Ital., vol. 1, p. 32, 1869. Change of name for Platystoma MElgen, 1803, not of KLein, 1753 (pre-Linnean and not since used in the Mollusca). Type, Musca seminationis Linners. Equals Platystoma Meigen, 1803.

Megalomyia Bigot, Bull. Soc. Ent. France for 1880, p. 5, 1880. 2 species. Type, Acanthomera seticornis Wiedemann, the first species, by present designation. Equals Pantophthalmus ThunberG, 1819.

- Megamerina Rondani, Dipt. Ital. Prodr., vol. 4, p. 10, 1861. ('hange of name for Lissa Meigen, 1826, not of Leach, 1815. Type, Ocyptera dolium Fabricils. Syn., Lissa Meiges, 1826, preoccupied.

$\checkmark$ Megametopon Giglio-Tos, Boll. Mus Zool. Univ. Torino, vol. 6, No. 108, p. 5, Sept. 20, 1891. No species. In Mem. Acad. Sci. Torino for 1892, 1 species. Type, Ophromyia nasica Williston. Syn., Ophromyia Williston, December, 1891.

$\checkmark$ Megaparia WulP, Biol. Centr.-Amer., Dept., vol.2, p. 240,1891. 1 species. Type, Megaparia venosa WulP.

- Megaphthalma Becker, Berliner Ent. Zeitschr., vol. 39, p. 105, 1894. 2 species. Type, Cordilura pallida FALLEN, the first species, by original designation.

- Megapollyon W ALKer, List Dipt. Brit. Mus., vol. 6, p. 452,1854. 13 species. Type, Microstylum acutirostre Loew, the first species, by present designation. Equals Microstylum Macquart, 1838.

$\checkmark$ Megaprosopus Macquart, Dipt. Exot., vol.2, pt. 3, p. 83, 1843. 1 species. Type, Megaprosopus rufiventris Macquart.

- Megarhina St. Fargeau and Serville, Eneycl. Meth., vol. 10, pt. 2, p. 585, 1828. 1 species. Type, Limnobiu longirostris Meigen. Syn., Helius St. Fargeau and Serville, 1828; Leptorhina Stephens, 1829; Rhamphidia Meigen, 1830.

- Megarhinus Desvoidy, Mem. Soc. Hist. Nat. Paris, vol. 3, p. 412, 1827. 1 species. Type, Culex hamorihoidalis Fabricus. Syn., Lynchiella Lanille, 1904.

Megaselia Ron1danı, Dipt. Ital. Prodr., vol. 1, p. 137, 1856. 1 species. Type, Phora costalis von Roser (as crassineura, new species). Syn., Trisometopia LIOY, 1864; Aphiochatu Brues, 1903.

Meghyperus LoEw, Ent. Zeit. Stettin, vol. 11, p. 303, 1850. 1 species. Type, Meghyperus sudeticus LoEw.

$\checkmark \quad$ Megistopoda Macquart, Ann. Soc. Ent. France for 1852, p. 332, 1852. 1 species. Type, Megistopoda pilatei Macevart.

$\checkmark$ Megistostylus Bigot, Ann. Soc. Ent. France for 1859, p. 215, 1859. 1 species. Type, Psilopus crinicornis Wiedemans.

Meigenia Desvorny, Essai Myod., p. 198, 1830. 2 species (as 11). Type, Tachina foralis Fallen (equals the last four supposed species), by designation of Desvoiny, Hist. Nat. Dipt., vol. 1, 1863, p. 1065. Syn., Spylosia Ronidani, 1856; Diplomera Lioy, 1864; Tenuicera PANdelle, 1896.

Meigeniella Coqullett, Proc. U. S. Nat. Mus., vol. 25, p. 104, 1902. 1 species. Type, Meigeniella hinei Cocoullettr.

Melaleuca WulP, Biol. Centr.-Amer., Dept., vol. 2, p. 247, 1891. 1 species. Type, Melaleuca spectabilis WULP.

$\checkmark$ Melanochelia Rondani, Atti Soc. Ital. Sci. Nat., vol.9, p. 5, 1866. 1 species. Type, Limnophora exsurla Pandelle (as Aricia surda Zetterstedt). Equals Limnophora Desvoidy, 1830.

- Melanoconion Theobali, Monogr. Culic., vol. 3, p. 238, 1903. 6 species. Type, Culex atratus Theobald, the first species, by designation of Dyar, Proc. Ent. Soc. Washington, vol. 7, 1905, p. 49. Syn., Mochlostyrax Dyar and KNaB, April 15, 1906.

$\checkmark$ Melanodexia Williston, N. Amer. Fanna, No. 7, p. 256, 1893.1 species. Type, Melanodexia tristis WiLliston. 
Melanogaster Rondan1, Dipt. Ital. Prodr., vol. 2, p. 166, 1857. 4 species. Type, Melanogaster nubilis RonDan1, the first species, by present designation. Equals Chrysogaster Meigen, 1800.

Melanophora MeIgen, Illiger's Mag., vol. 2, p. 279, 1803. 1 species. Type, Musca

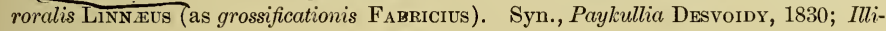
geria Desvoldy, 1830.

Melanophrys Williston, Trans. Amer. Ent. Soc., vol. 13, p. 305, 1886. 1 species. 'Type, Tachina insolita WALKER (as flavipennis, new species). Syn., Atropharista TownSEND, 1892.

Melanostoma Schiner, Wien. Ent. Monatschr., vol. 4, p. 213, 1860. 1 species. Type, Musca mellina Linneus.

- Melieria Desvoidy, Essai Myod., p. 715, 1830. 5 species. Type, Musca crassipennis Fabricius (as gangrenosa Desvordy), the first species, by designation of Rondani, Dipt. Ital. Prodr., vol. 1, 1856, p. 108.

- Melina Desvordy, Essai. Myod., p. 695, 1830. 1 species. Type, Melina riparia Desvoidy. Equals Chetocera Desvordy, 1830.

$\checkmark$ Melinda Desvoidy, Essai Myod., p. 439, 1830. 2 species (as 7). Type, Musca cognata Meigen (equals six of the supposed species), by present designation. Equals Onesia Desvordy, 1830.

Melithreptus LoEw, Isis von Oken for 1840, p. 577, 1840. Change of name for Sphrrophoria St. FARgeau and Serville, 1828, because of nearly identical older names. Type, Musca scripta Linndus. Equals Sphærophoria St. Fargeau and SERVILLE, 1828.

$\checkmark$ Melitrophus WaLker, Ins. Britt., Dipt., vol. 3, p. xxi, 1856. Change of name for Melithreptus LoEw, 1840, preoccupied by a genus of birds. Type, Musca scripta LiNnæus. Equals Sphærophoria St. Fargeau and Serville, 1828.

- Melophagus Latreille, Hist. Nat. Crust. et Ins., vol. 3, p. 466, 1802. 1 species. Type, Hippobosca ovina Linn xus. Syn., Melophila Nitzsch, 1818.

- Melophila Nitzsch, Germ. Mag. Ent., vol. 3, p. 311, 1818. 1 species. Type, Hippobosca ovina Linnæus. Equals Melophagus Latreille, 1802.

- Melpia W Alker, Ins. Saund., Dipt., p. 8, 1850. 9 species. Type, Pangonius fulvithorax Wiedemann, the third species, by present designation. Equals Pangonius Latreille, 1802.

Melusina Meigen, Nouv. Class. Mouch., p. 19, 1800.* No species. Atractocera Meigen, 1803, is a change of name. Type, Simulium ornatum Meigen. Syn., Simulium Latreille, 1802; Atractocera Meigen, 1803.

Merapioidus Віяот, Bull. Soc. Ent. France for 1879, p. 50, 1879.1 species. Type, Merapioidus villosus BıGot.

Meriania Desvoldy, Essai Myod., p. 69, 1830. 2 species (as 3). Type, Musca puparum Fabricius (as silvatica, new species), the first species, by designation of Desvoidy, Hist. Nat. Dipt., vol. 1, p. 169, 1863. Syrr., Platychira Rondani, 1856.

Mericia Desvordy, Essai Myod., p. 64, 1830. 1 species. Type, Mericia erigonea Desvoidy. Equals Ernestia Desvoldy, 1830.

Merodina Macquart, Hist. Nat., Dipt., vol. 2, p. 378, 1835. 1 species. Type, Dacus podagrica Fabricius. Equals Richardia Desvoidy, 1830.

Merodon Meigen, Illiger's Mag., vol. 2, p. 274, 1803. 2 species. Type, Syrphus clavipes FABricius (as curvipes Fabricius), the second species, by designation of Westwoon, Intr., vol. 2, Synops., 1840, p. 137. Equals Lampetia Meigen, 1800.

Meromacrus Rondan1, Studi Ent., vol. 1, p. 70, 1848. 1 species. Type, Meromacrus ghilianii Rondanı. Syn., Plagiocera Macquart, 1842, preoccupied; Pteroptila LoEw, 1865.

Meromyza Meigen, Syst. Beschr. Zweifl. Ins., vol. 6, p. 163, 1830. 3 species. Type, Musca saltatrix Linnæus, the third species, by designation of Westwoon, Intr., vol. 2, Synops., 1840, p. 147. 
V Meroplius Rondanı, Bull. Soc. Ent. Ital., vol. 6, p. 170, 1874. 1 species. Type, Nemopoda stercoraria Desvordy. Equals Nemopoda Desvordy, 1830.

$\checkmark$ Merosargus Loew, Verh. Zool.-Bot. Ges. Wien, vol. 5, p. 144, 1855. 4 species. Type, Sargus obscurus Wiedemans, the third species, by original designation.

$\checkmark$ Mesembrina Melgen, Syst. Beschr. Zweifl. Ins., vol. 5, p. 10, 1826.2 species. Type, Musca meridiana Liswæus, the first species, by designation of Westwood, Intr., vol. 2, Synops., 1840, p. 141. Syn., Metamesembrina Townsenv, 1908.

$\checkmark$ Mesembrinella Giglio-Tos, Boll. Mus. Zool. Univ. Torino, vol. 8, No. 147, p. 4, 1893. 1 species. Type, Mesembrinella bicolor (Higlio-Tos.

$\checkmark$ Mesembrius Rondani, Dipt. Ital. Prodr., vol. 2, p. 50, 1857. 1 species. Type, Helophilus peregrinus Loew. Equals Helophilus Meneien, 1822.

$\checkmark$ Mesogramma Loew, Berliner Ent. Zeitschr., vol. 9, p. 157, 1865. 12 species. Type, Syrphus gurgus WALKER (as parula, new species), the first species, by designation of Williston, Synops. N. Amer. Syrph., 1886, p. 98. Equals Toxomerus Macquart, 1855.

Mesograpta Loew, Berliner Ent. Zeitschr., vol. 16, p. 290, 1872. Change of name for Mesogramma LoEw, 1865, under the mistaken idea that the latter is preoccupied by a genus of plants. Type, Syrphus gurges WaLker. Equals Toxomerus Macquart, 1855.

$\checkmark$ Mesophila Walker, List Dipt. Brit. Mus., vol. 4, p. 1157, 1849. 1 species. Type, Ceratophya fuscipennis Macevart. Equals Microdon Meigen, 1803.

$\checkmark$ Mesorhaga Schiner, Reise Novara, Zool., vol. 2, Dipt., p. 217, 1868. 1 species. Type, Mesorhaga tristis Schiner. Syn., Aptorthus Aldrich, 1893.

$\checkmark$ Messala Curtis, Brit. Ent., p. 581, 1836. 1 species. Type, Macrocera hybrida Meigen (as saundersii, new species). Equals Bolitophila Meigen, 1818.

$\checkmark$ Metachæta Coqulllett, Journ. N. Y. Ent. Soc., vol. 3, p. 98, 1895. 1 species. Type, Tachina helymus WALKER (as atra, new species).

$\checkmark \quad$ Metachela Coquilletr, Proc. Ent. Soc. Washington, vol. 5, p. 253, 1903. 1 species. Type, Hemerodromia collusor Mela NDER.

Metacosmus Coqullett, West. Amer. Sci., vol. 7, p. 220, 1891. 1 species. Type, Metacosmus exilis Conunletetr.

C Metadexia Coquillett, Journ. N. Y. Ent. Soc., vol. 7, p. 220, 1899. 1 species. Type, Metarlexia tricolor Convillett.

Metadiplosis Felt, 23d Rep. State Ent. N. Y., p. 406, 1908.. 1 species. Type, Metadiplosis spinosu FELT.

Metadoria Brauer and Bergenstam, Denkschr. Kais. Akad. Wiss. Wien, vol. 60, p. 117, 1893. 1 species. Type, Phorocera barbata Bigot (as mexicana, new species).

Syn., Hemiargyru Tow veve, 1908.

Metamesembrina Townsend, Smiths. Misc. Coll., vol. 51, p. 50, 1908.1 species. Type, Musca meridiana Linveus. Equals Mesembrina Meigen, 1826.

Metapelastoneurus Aldrich, Kansas Univ. Quart., vol. 2, p. 152, 1894. 1 species. Type, Metapelastoneurus kansensis Aldrich. Equals Pelastoneurus Loew, 1861.

$\checkmark$ Metaphragma Coquillett, Journ. N. Y. Ent. Soc., vol. 2, p. 97, 1894. 1 species. Type, Yestomyza planiceps Loew.

Metaphyto Coquilletr, Rev. Tach., p. 36, 1897. 1 species. Type, Metaphyto genalis Coquillett.

Metaplagia Coquilletr, Journ. N. Y. Ent. Soc., vol. 3, p. 102, 1895. 1 species. Type, Metaplagia occiclentalis Coqullettr.

Metapogon Coquillett, Proc. Ent. Soc. Washington, vol. 6, p. 181, 1904. 2 species. Type, Metapogon gilvipes Coqullett, the first species, by original designation.

Metatrichia Coquillett, Fnt. News., vol. 11, p. 500, 1900. 1 species. Type, Scenopinus bulbosus Osten Sacken.

Methypostenu Townsend, Smiths. Misc. Coll., vol. 51, p. 67, 1908. 1 species. Type, Hypostena barbata Coquilletт. Equals Tachinophyto Townsend, 1892. 
Metonia Meigen, Illiger's Mag., vol. 2, p. 280, 1803. 1 species. Type, Musca lencocephala Rossi. Syn., Ophelia Desvoidy, 1830; Araba Desvordy, 1830; Argyria Desvoldy, 1863; Argyrella Desvoidy, 1863; Arabella Desvoidy, 1863; Anicia Desvordy, 1863.

Metopilla Rondani, Dipt. Ital. Prodr., vol. 3, p. 210, 1859. Originally published as a synonym of Heteropterina MArquart, with 1 species. Type, Heteropterina multipunctata Rondani. Equals Taxigramma Perris, 1852.

Metopina Macquart, Hist. Nat., Dipt., vol. 2, p. 666, 1835.1 species. Type, Phora galeata Haliday. Syn., Leptophora Six, 1878; Drepanophora Strobl, 1880.

Metriocnemus Wulp, Tijdschr. Ent., vol. 17, p. 136, 1874. 6 species. Type, Chironomus albolineatus MEIGEN, the first species, by present designation.

Miastor Meinert, Nat. Tidsskr. for 1864, p. 42, 1864.* 1 species. Type, Miastor metraloas MEINERT.

Micetoica Rondani, Dipt. Ital. Prodr., vol. 4, p. 12, 1861. Change of name for Nycetobia Meigen, 1818, under the mistaken idea that it is preoccupied by Mycetobia Dumeril, 1806, used for a section in Coleoptera. Type, Mycetobia pallipes Meigen. Equals Mycetobia Meigen, 1818.

Michogaster Macquart, Hist. Nat., Dipt., vol. 2, p. 483, 1835. 3 species. Type, Cephalia femoralis Wiedemann, the first species, by designation of Schiner, Reise Novara, Zool., vol. 2, 1868, p. 255. Syn., Conopsida Maceuart, 1851.

Micraëdes Coquillett, Proc. Ent. Soc. Washington, vol. 7, p. 185, 1906.1 species. Ty pe, Micraëdes bisulcatus Coquillett.

Micraptoma Westwood, Intr., vol. 2, Synops., p. 136, 1840. 1 species. Type, Musca segnis Linneus. Equals Zelima Meigen, 1800.

Mirrocera Meigen, Illiger's Mag., vol. 2, p. 273, 1803. No species. In his Syst. Beschr., vol. 4, 1824, p. 19, he states that it equals Pipunculus Latreille, 1802. Type, Pipunculus campestris Latreille. Equals Dorilas Meigen, 1800.

Microcera ZetTenstedt, Ins. Lapp., p. 572, 1838. 1 species. Type, IIeleodromia immaculata Haliday (as rostrata, new species). Not Ficrocera Meigen, 1803. Equals Heleodromia HaLinar, 1833.

Microcera Lioy, Atti Inst. Veneto, ser. 3, vol. 9, p. 906, 1864. 1 species (as 2). Type, Musca ciliata Fabricius. Equals Hydrotæa Desvoidy, 1830.

Microchætina Wulp, Biol. Centr.-Amer., Dipt., vol. 2, p. 240, 1891. 1 species. Type, Microchrtina cinerea WULP.

Microcheilosia Macquart, Ann. Soc. Ent. France for 1855, p. 183, 1855.1 species. Type, Microcheilosia nitida Macquart. Equals Rhinophora Desvoidy, 1830.

Microchira Brauer and Bergenstamm, Denkschr. Kais. Akad. Wiss. Wien, vol. 60, p. 128, 1893.1 species. Type, Microchira mexicana Brauer and Bergenstamm. Equals Paradidyma Brauer and Bergenstamm, 1891.

Microchrysa Loew, Verh. Zool.-Bot. Ges. Wien, vol. 5, p. 146, 1855. 1 species. Type, Musca polita Linneus. Equals Chrysomyia Macquart, 1834.

Microdon Meigen, Illiger's Mag., vol. 2, p. 275, 1803. 1 species. Type, Musca mutabilis Linneus. Syn., Aphritis Latreille, 1805; Ceratophya Wiedemann, 1824; Chymophila Macquart, 1834; Dimeraspis Newman, 1838; Mesophila Walker, 1849; Ubristes WALKER, 1852.

Microdromya B1Got, Ann. Soc. Ent. France for 1857, p. 557, 1857. No species. The characters given apply to Tachydromia oratorio FALLEN, which may be taken as the type. Type Tachydromia oratorio FALLEN.

Micropalpis Macquart, Rec. Tràv. Soc. Sci. Lille for 1834, p. 316, 1834. 6 supposed species. Type, Tachina comta FALLEN (the first, third, and fourth supposed species), by designation of WEstwoon, Intr., vol. 2, Synops., 1840, p. 138 (as fulgens Meigen). Equals Bonnetia Desvoidy, 1830.

Micropeza Meigen, Illiger's Mag., vol. 2, p. 276,.1803. 1. species. Type, Musca corrigiolata Linnæus. Equals Tylos Meigen, 1800. 
Microphorus Macquar', Ins. Dipt. Nord France, separata, p. 139, 1827. 3 supposed species. Type, Microphorus velutimus Macquart, the second species, by designation of Rondan1, Dipt. Ital. Prodr., vol. 1, 1856, p. 151.

Microphthalma MIACQuar', Dipt. Exot., vol. 2, pt. 3, p. 84, 1843.1 species. Type, Tachina disjuncta Wiedemann (as nigra, new species). Syn., Amesia Desvordy, 1863.

$\checkmark$ Microprosopa Becker, Berliner Ent. Zeitschr., vol. 39, p. 147, 1894. 9 species. Type, Cordilura hrmorrhoidalis MeIgen, the eighth species, by original designation.

$\checkmark$ Microstylum Macquar', Dipt. Exot., vol. 1, pt. 2, p. 26, 1838.10 species. Type, Dasypogon venosus Wiedemann, the first species, by designation of Back, Trans. Amer. Ent. Soc., vol 35, p. 213, 1909. Syn., Megapollyon W Alker, 1854.

- Microtachina Мı, Wien. Ent. Zeit., vol. 11, p. 116, 1892. 1 species. Type, Tachina nympharum Rondani. Equals Exorista Meigen, 1803.

V Microtrichomma Giglio-Tos, Boll. Mus. Zool. Univ. Torino, vol. 8, no. 158, p. 1, 1893. 3 species. Type, Nemoræa forreri Wulp, the first species, by present designation. Equals Arthrochæta Brauer and Bergenstamu, 1889.

- Mikimyia Bigot, Bull. Soc. Ent. France for 1884, p. 29, 1884. 1 species. Type, Toxotrypana curvicauda Gerstacker (as furcifera, new species). Equals Toxotrypana Gerstæcker, 1860.

- Milesia Latreille, Hist. Nat. Crust.' et Ins., vol. 14, p. 361, 1805. 2 species. Type, Musca diophthalma Linneus, the first species, by designation of Rondani, Nuovi Ann. Sci. Nat. (Bologna), 1844, p. 455. Equals Spilomyia Meigen, 1803. (Milesia of authors equals Sphixea Rondani).

- Milesiformis Rondani, Nuovi Ann. Sci. Nat. (Bologna) for 1844, p. 454, 1844. 1 species. Type, Musca scita Harris (as milesiformis Fallen). Equals Tropidia Meigen, 1822 .

- [Milichia Meigen, Syst. Beschr. Zweifl. Ins., vol. 6, p. 131, 1830. 2 species. Type, Milichia speciosa Meigen, the first species, by designation of Westwood, Intr., vol. 2, 1840, Synops. p. 151. Not American.] (The American species belong to Rhynchomilichia Hendel.)

Milichiella Giglio-Tos, Ann. Soc. Ent. France for 1895, p. 367, 1895. 1 species. Type, Milichiella tosi Becker (as Tephritis argentea FABricius). Syn., Ophthalmomyia Williston, 1896; Stenoporomyia Hendel, 1903.

$\checkmark$ Mima Meigen, Syst. Beschr. Zweifl. Ins., vol. 2, p. 175, 1820. 1 species. Type, Anthrax phxoptera Meigen. Syn., Exoprosopa Macquart, 1840; Litorhynchus MaCQUarT, 1840; Trinaria Mulsant, 1852; Argyrospyla Rondanı, 1856; Heteralonia RonDANI, 1864; Defilippia Lioy, 1864.

$\checkmark$ Mimosciara Rondani, Mem. Sec. Ditt. Ital., p. 25, 1840**2 species. Type, Mimosciara molobrina Rondani, the first species, by present designation. Equals Lestremia MACQUART, 1826.

Q Minettia Desvoidy, Essai Myod., p. 646, 1830. 7 species. Type, Musca longipennis Fabriscrits (as luctuosa, new species), the first species, by present designation. Equals Sapromyza FaLlen, 1810.

- Misgomyia Coquillett, Proc. Ent. Soc. Washington, vol. 9, p. 145, 1908. 1 species. Type, Misgomyia obscura Coqullett.

$\checkmark$ Mixogaster M.Acquart, Dipt. Exot., vol. 2, pt. 2, p. 14, 1842. 1 species. Type, Mixogaster conopsoides MaceUar'T.

$\checkmark$ Mixtemyia Macquart, Hist. Nat., Dipt., vol. 1, p. 491, 1834. 1 species. Type, Paragus quảdrifasciatus SAY. Equals Spilomyia MIEIGen, 1803.

$\checkmark \quad$ Mochlonyx Loew, Ent. Zeit. Stettin, vol. 5, p. 121, 1844. 1 species. Type, Corethra velutina Ruthe. Equals Corethra MEIGEN, 1803.

Mochlosoma Brauer and Bergenstamm, Denkschr. Kais. Akad. Wiss. Wien, vol. 56, p. 126, 1889.1 species. Type, Mochlosona validum Brader and Bergexstami.

$\checkmark$ Mochlostyrax Drar and KNaB, Journ. N. Y. Ent. Soc., vol. 14, p. 223, 1906. 6 species. Type, Mochlostyrax caudelli Dyar and KNaB, the fifth species, by original designation. Equals Melanoconion TheовaLd, July 25, 1903. 
Mochtherus Loww, Linn. Ent., vol. 4, p. 58, 1849 . 7 species. Type, Asilus pallipes Meigen, the fifth species, by present designation. Not Mochtherus Schmidt-Goebel, 1846. Equals Heligmonevra Bigot, 1858.

Molobrus Latreille, Hist. Nat. Crust. et Ins., vol. 14, p. 288, 1805. 1 species. Type, Tipula thomæ Linneus (as the "Tipule de Thomas"). Equals Lycoria MeIGeN, 1800.

Molophilus Curtis, Brit. Ent., p. 444, 1833. 1 species. Type, Erioptera atra Meigen (as brevipennis, new species).

Molynocœlia Giglio-Tos, Boll. Mus. Zool. Univ. Torino, vol. 8, No. 158, p. 11, 1893. 1 species. Type, Molynoccelia lutea Giglio-Tos.

Mongoma Westwood, Trans. Ent. Soc. Lond. for 1881, p. 364, 1881. 1 species. Type, Mongoma fragillima Westwood.

Morellia Desvoldy, Essai Myod., p. 405, 1830. 3 species (as 4). Type, Musca hortorum FALLEN (as agilis and horticola, new species), the first species, by present designation. Syn., Alina Desvoidy, 1863; Camilla Desvoidy, 1863.

Morimna Walker, Ins. Saund., Dipt., p. 104, 1851. 1 species. Type, Morimna mallophorides WALKer. Equals Lastaurus Loew, 1851.

Morinia Desvoldy, Essai Myod., p. 264, 1830. 2 species (as 5). Type, Musca melanoptera FALLen (as velox, new species), the first species, by designation of RoNDani, Dipt. Ital. Prodr., vol. 5, 1862, p. 159. Syn., Anthracomya Rondan1, 1856.

- Morphomya Rondanı, Dipt. Ital. Prodr., vol. 1, p. 83, 1856. 1 species. Type, Musca tachinoides FALLEN.

- Moschusa Desvordy, Hist. Nat. Dipt., vol. 2, p. 139, 1863. 1 species. Type, Tachina polyodon Meigen. Equals Amobia Desvoidr, 1830.

Mosillus Latreille, Hist. Nat. Crust. et Ins., vol. 14, p. 389, 1805. 1 species. Type, Syrphus subsultans FABricius (as arcuatus, new species). Syn., Gymnopa Fallen, 1820; Glabrinus Rondan1, 1856.

- Mosina Desvoldy, Essai Myod., p. 670, 1830. 7 species. Type, Musca pubera Linnesus, the first species, by present designation. Equals Cordilura Fallen, 1810.

- Mufetia Desvoiny, Essai Myod., p. 431, 1830. 1 species. Type, Mufetia autissiodorensis Desvoidy. Equals Calliphora Desvoidy, 1830.

$\checkmark$ Mulsantia Desvoidy, Hist. Nat. Dipt., vol. 2, p. 557, 1863. 4 species (as 30). Type, Sarcophaga hrmorrhoa Meigen (as campestris, new species), the first species, by present designation. Equals Hartigia Desvoldy, 1863.

Murana Meigen, Syst. Beschr. Zweifl. Ins., vol. 4, p. 223, 1824. 1 species. Type, Trixa alpina Meigen. Equals Trixa Meigen, 1824.

Musca Linnæus, Syst. Nat., 10th ed., p. 589, 1758. 100 species. Type, Musca domestica Linnæus, the fifty-fourth species, by designation of Macquart, Ins. Dipt. Nord France, Ather., 1834, p. 20. Syn., Plaxemya Desvoidy, 1830; Byomya DesvoIDY, 1830.

Muscina Desvordy, Essai Myod., p. 406, 1830. 4 species (as 6). Type, Musca stabulans F ALLEN, the second species, by present designation. Syn , Blissonia Desvoidy, 1863; Pararicia Brauer and Bergenstamm, 1891.

$\checkmark$ Musciosoma Lioy, Atti Inst. Veneto, ser. 3, vol. 9, p. 908, 1864. Change of name for Hylemya Desvoidy, 1830, under the mistaken impression that it is too near Hyalomya Desvoidy, 1830. Type, Musca strigosa Fabricius. Equals Hylemya Desvoidy, 1830.

Muscopteryx Townsend, Can. Ent., vol. 24, p. 170, 1892. 1 species. Type, Muscopteryx chrtosula Townsend.

Mutiloptera Coquilletт, Proc. Ent. Soc. Washington, vol. 9, p. 147, 1908. 1 species. Type, Mutiloptera apicalis Coquillett.

- Mya Rondan1, Nuovi Ann. Sci. Nat. (Bologna) for 1850, p. 175, 1850. 3 species. Type, Musca segmentaria Fabricius, the third species, by present designation. Equals Lucilia Desvoldy, 1830. 
Myantha Rondan, Dipt. Ital. Prodr., vol. 1, p. 95, 1856. 1 species. Type, Musca canicularis Linneus. Equals Fannia Desvoidy, 1830.

4. Mycetaulus Loew, Dipt. Beitr., vol. 1, p. 37, 1845. 1 species. Type, Geomyza bipunctata FALLen (as hoffmeisteri, new species). Syn., Brachygaster MElGEN, 1826, preoccupied.

$\checkmark$ Mycetia Desvoldy, Essai Myod., p. 805, 1830. 5 species. Type, Copromyza equina FaLlen (as vulgaris, new species), the fourth species, by present designation. Equals Copromyza FALLen, 1810.

- Mycetina Rondan, Dipt. Ital. Prodr., vol. 1, p. 195, 1856.1 species. Type, Mycetophila flavipes Macquart. Not Mycetina Mulsant, 1846. Equals Fungivora Melicen, 1800.

$\checkmark /$ Mycetobia Meigen, Syst. Beschr. Zweifl. Ins., vol. 1, p. 229, 1818. 2 species. Type, Mycetobia pallipes Meigen, the first species, by designation of Westwood, Intr., vol. 2, Synops., 1840, p. 127. Syn., Micetoica Roxnaxi, 1861.

$\checkmark$ Mycetophila Melgen, Illiger's Mag., vol. 2, p. 263, 1803. 2 species. Type, Mycetophila agarici OLıvier, the second species, by designation of Johannsen, Gen. Ins., Dipt., Mycet., p. 116, 1909. Equals Fungivora Meigen, 1800.

- Mycodiplosis RÜbsiamen, Ent. Nach., vol. 21, p. 186, 1895. 1 species. Type, Diplosis coniophaga W1NNERTz.

$\checkmark$ Mycomya Rondanı, Dipt. Ital. Prodr., vol. 1, p. 194, 1856. 1 species. Type, Sciophila marginata MEIGEN.

$\checkmark$ Mycothera Winnertz, Verh. Zool.-Bot. Ges. Wien, vol. 13, p. 913, 1863. 2 species. Type, Mycetophila dimidiata STEGer, the first species, by designation of JoHannsen, Gen. Ins., Dipt., Mrcet., 1909, p. 111.

$\checkmark$ Mycozetza Ronisixı, Dipt. Ital. Prodr., vol. 4, p. 12, 1861. Change of name for Mycetina Rondani, 1856, not of Mulsant, 1846. Type, Mycetophila flaripes Macevart. Equals Fungivora Meigen, 1800.

$\checkmark \quad$ Mydæa Desvoiny, Essai Myod., p. 479, 1830. 7 species (as 9). Type, Musca pagana Fabricius (as scutellaris, new species), the fifth species, by designation of CoquilletT, Journ. N. Y. Ent. Soc., vol. 9, 1901, p. 139. Syn., Aricia Desvolny, 1830; IIelina Desvoldy, 1830; Mydina Desvordy, 1830; Spilogaster Maceuart, 1835; Myospila Rondan1, 1856; Yetodesiu Rondanı, 1861; Aspilia Rondan1, 1866.

Mydas F Frricius, Ent. Syst., vol. 4, p. 252, 1794. 3 species. Type, Musca clarata Drury, the first species, by designation of Latreille, Consid. Général., 1810, p. 443. Syn., Phyllomydas Bigot, 1880.

- Mydina Destoldy, Essai Myod., p. 495, 1830. 16 species (as 22). Type, Musca quadrum Fabricius (as dispar, new species), the fourth species, by designation of Coquillett, Journ. N.Y. Ent. Soc., vol. 9, 1901, p. 139. Equals Mydæa Desrordr, 1830.

$\checkmark$ Myelaphus Bıяот, Bull. Soc. Ent. France for 1882, p. 91, 1882. 1 species. Type, Myelaphus melas Bigot.

Myennis Desvordy, Essai Myod.,p. 717, 1830. 1 species. Type, 1/usca octopunctata Coquebert (as fasciata, new species).

$\checkmark \quad$ Myiomima Brauer and Bergenstamu, Denkschr. Kais. Akad. Wiss. Wien, vol. 56, p. 119, 1889.1 species. Type, Myiomima sarcophagina Brauer and Bergenstamu.

$\checkmark \quad$ Myiopharus Brauer and Bergenstanin, Denkschr. Kais. Akad. Wiss. Wien, vol. 56, p. 161, 1889. 1 species. Type, Myiopharus metopia Brauer and Bergenstamm.

Myiophasia Brauer and Bergenstama, Denkschr. Kais. A kad. Wiss. Wien, vol. 58, p. 362, 1891. 1 species. Type, Clytia atra Desvoldy (as Tachina anea Wienemans, 1830, not of Meigen, 1824). Syn., Phasioclista Townsend, 1891; Ennyomma Townsend, 1891.

Myioscotiptera Giglio-Tos, Boll. Mus. Zool. Univ. Torino, vol. 8, No. 147, p. 2, 1893. 1 species. Type, Myioscotiptera cincta Giglio-Tos.

Hyobia. Desvoldy, Essai Myod., p. 98, 1830. 2 species (as 7). Type, Tachina inanis FaLlen (equals the first three and last two supposed species), by designation of 
Westwood, Intr., vol. 2, 1840, Synops., p. 139. Not Nyobia Heyden, 1826. Equals Leskia Destordy, 1830.

Myocera Desvordy, Essai Myod., p. 328, 1830. 2 species (as 6). Type, Musca ferina Fallen, the first two supposed species, by designation of BraUer, Verh. Zool.Bot. Ges. Wien, vol. 43, 1893, p. 507. Equals Phorostoma Desvoidy, 1830.

Myochrysa Rondani, Dipt. Ital. Prodr., vol. 4, p. 11, 1861. Change of name for Chrysomyia MaceUart, 1834, under the mistaken impression that it is preoceupied by Chrysomya Desvomy, 1830. Type, Musca polita Linneus. Equals Chrysomyia MACQUART, 1834 .

Myodina Desvordy, Essai Myod., p. 727, 1830. 1 species. Type, Musca vibrans Linneus (as urticx Fabricius). Equals Ortalis Fallen, 1810.

Myoleja Rondani, Dipt. Ital. Prodr., vol. 1, p. 112, 1856. 1 species. Type, Tephritis lucida Fallen. Equals Acidia Desvoidr, 1830.

Myolepta Newman, Ent. Mag., vol. 5, p. 373, 1838. 1 species. Type, Nusca luteola Gmelin. Syn. Xylotreja Rondani, 1844.

Myopa Fabricius, Syst. Ent., p. 798, 1775. 4 species. Type, Conops buccata LiNN Stomoxoides SCH FFFER, 1766.

Myopella Desvordy, Dipt. Env. Paris, Myop., p. 19, 1853.* 3 species (as9). Type, Myopa stigma Meigen (equals the last three supposed species), by present designation. Equals Stomoxoides SCH ÆFFER, 1766.

- Myophora Destoiny, Essai Myod., p. 337, 1830. Change of name for Sarcophaga MEIGEN, 1826, because all of the species are not flesh-eaters. Type, Nusca carnaria Linneus. Equals Sarcophaga Meigen, 1826.

$\checkmark$ Myopina Desvordy, Dipt. Env. Paris, Myop., p. 39, 1853.* 1 species. Type, Мyopa variegata MeIgen. Equals Stomoxoides SCH ÆFFer, 1766.

Myospila Rondani, Dipt. Ital. Prodr., vol. 1, p. 91, 1856. 1 species. Type, Musca meditabunda Fabricius. Equals Mydæa Desvoidy, 1830.

Myothyria Wulp, Biol. Centr.-Amer., Dipt., vol. 2, p. 208, 1890. 3 species. Type, Myothyria majorina WuLP, the second species, by present designation.

Myrmecomya Desvoidy, Essai Myod., p. 721, 1830. 2 species. Type, Cephalia rufipes MEIGEN (as formicaria, new species), the first species, by present designation. Equals Cephalia Meigen, 1826.

Mystacella Wulp, Biol. Centr.-Amer., Dipt., vol. 2, p. 51, 1890. 12 species. Type, Mystacella solitg WULP, the fifth species, by present designation.

Mystacomyia Giglio-Tos, Boll. Mus. Zool. Univ. Torino, vol. 8, No. 158, p. 4, 1893. 1 species. Type, Mystacella rubriventris Wulp.

Mythicomyia Coquilletr, Ent. News, vol. 4, p. 208, 1893. 1 species. Type, Mythicomyia rileyi Coqu ILLETT.

Myxexorista Brauer and Bergenstami, Denkschr. Kais. Akad. Wiss. Wien, vol. 58, p. 331, 1891. 9 species. Type, Nusca libatrix Panzer, the eighth species, by designation of Brauer and Bergenstamm, Verh. Zool.-Bot. Ges. Wien, vol. 43, 1893, p. 479. Equals Zenilla Desvordy, 1830.

Myxosargus Brauer, Denkschr. Kais. Akad. Wiss. Wien, vol. 44, p. 77, 1882. 1 species. Type, Myxosargus fasciatus Brauer.

Myzomyia Blanchard, Compt. Rend. Soc. Biol., vol. 54, p. 795, July 4, 1902. Change of name for Grassia Theobald, June 16, 1902, not of Fisch, 1885. Type, Anopheles rossii Giles. Syn., Grassia Theobald, June 16, 1902, preoccupied.

Myzorhynchella Theobald, Monogr. Culic., vol. 4, p. 78, 1907. 1 species. Type, Myzorhynchella nigra Theoвald. Equals Anopheles Meigen, 1818.

Napæa Desvoiny, Essai Myod., p. 799, 1830. 2 species. Type, Ephydra coarctata FALLEN (as stagnicola, new species), the first species, by designation of Westwood, Intr., vol. 2, Synops., 1840, p. 153. Syn., Parydra Stenhammar, 1843; Hygrophila Lioy, 1864; Falosoma Lioy, 1864; Ephydrosoma Lioy, 1864. 
Napomyza Haliday, in Westwood's Intr., vol. 2, Synops., p. 152, 1840. 1 species. Type, Phytomyza elegans Meigen (as festina Meigex). Syn., Dinevra Lioy, 1864.

Nausigaster Williston, Trans. Amer. Ent. Soc., vol. 11, p. 33, 1883. 1 species. Type, Nausigaster punctulata Williston.

$\checkmark$ Neæra Desvoidy, Essai Myod., p. 84, 1830. 1 species. Type, Tachinu albicollis Meigen (as immaculata, new species). Syn., Thapsia Desvoidy, 1863; Glaucophana Brauer and Bergenstami, 1891.

Neaspilota Osten Sacken, Cat. Dipt. N. Amer.,p. 192, 1878. Change of name for Aspilota Loew, 1873, not of Ferster, 1862. Type, Trypeta alba Loew. Syn., Aspilota LOEW, 1873, preoceupied.

$\checkmark$ Nebritus Coquilletr, Journ. N. Y. Ent. Soc., vol. 2, p. 98, 1894. 1 species. Type, Nebritus pellucidus CoquilletT.

- Nematocera Meigen, Syst. Beschr. Zweifl. Ins., vol. 1, p. 209, 1818. 1 species. Type, Nematocera bicolor Meigen. Equals Hexatoma Latreille, 1809.

- Nematoproctus Loew, Neue Beitr., vol. 5, p. 40, 1857. 2 species. Type, Chrysotus distendens MElGen (as Porphyrops annulatus MACQUART), the first species, by present designation.

- Nemochata Wulp, Biol. Centr.-Amer., Dipt., vol. 2, p. 38, 1888. 1 species. Type, Nemochrta dissimilis Wulp. Equals Archytas Jænnicke, 1867.

$\checkmark \quad$ Nemopoda Desvoldr, Essai Myod.,p. 743, 1830. 5 species. Type, Musca cylindrira FABR1c1us (as putris, new species), the first species, by designation of WEsTwoon, Intr., vol. 2, Synops., 1840, p. 148. Syn., Meroplius Rondan1, 1874.

- Nemoræa Desvoldy, Essai Myod., p. 71, 1830. 2 species (as 6). Type, Tachina pellucida MEIGEN (equaling the first three supposed species), by designation of Rondani, Dipt. Ital. Prodr., vol. 1, 1856, p. 64.

$\checkmark$ Nemosia Lioy, Atti Inst. Veneto, ser. 3, vol. 10, p. 78, 1864. 1 species. Type, Phora incrassata Meigen. Equals Hypocera Lioy, 1864.

v Nemotelus Geoffroy, Hist. Abrégée Ins., vol. 2, p. 542, 1762. 2 species (without names). Type, Musca pantherinu Lisneus, the first species, by designation of Latreille, Consid. General., 1810, p. 442 (as uliginosus and marginatus).

Neoascia Williston, Synops. N. Amer. Syrph., p. 111, 1886 . Change of name for Ascir Meigen, 1822, not of Scopoli, 1777. Type, Ascia floralis Meigen. Syn., Ascia MElGEN, 1822, preoceupied.

Neocerata Coquillett, Bull. 22, n. ser., Div. Ent., U. S. Dept. Agr., p. 47, 1900. 1 species. Type, Neocerata rhodophaga CoquilletT.

$\checkmark$ Neochauna Williston, Man. N. Amer., Dipt., p. 48, 1896. Change of name for Chauna Loew, 1847, not of Illiger, 1811. Type, Chauna variabilis Loew. Syn., Chauna Loew, 18+7, preoccupied.

Neocota Coquillett, Proc. U. S. Nat. Mus., vol. 18, p. 434, 1896.1 species. Type, Neocota weedi Coquillett.

$\checkmark \quad$ Neoculex Dyar, Proc. Ent. Soc. Washington, vol. 7, p. 45, 1905. 1 species. Type, Culex territans Whaker. Equals Culex Linneus, 1758.

Neoëmpheria Osten SAcken, Cat. Dipt. N. Amer., p. 9, 1878. Change of name for Empheria Winnertz, 1863, not of Hagen, 1856. Type, Sciophila striata Meigen. Syn., Empheria Winnertz, 1863, preoccupied.

Neö̈risticus Osten Sacken, Cat. Dipt. N. Amer., p. 81, 1878. Change of name for Eristicus Loew, 1848, not of Wesmel, 1844. Type, Asilus intermptus Macquart. Equals Eicherax Bigot, 1857.

$\checkmark \quad$ Neoëxaireta Osten Sacken, Cat. Dipt. N. Amer., p. 44, 1878. Change of name for Exaireta ScH1NER, 1867, under the mistaken impression that the latter is preoceupied. Type, Xylophagus spiniger W1EDEMAnN. Equals Exaireta Sch1Ner, 1867.

Neofischeria Townsend, Smiths. Misc. Coll., vol. 51, p. 74, 1908. 1 species. Type, Demoticus venatoris Coquillett (as flava, new species). Equals Demoticus Macquart, 1854. 
Neoglaphyroptera Osten SAcken, Cat. Dipt. N. Amer., p. 10, 1878. Change of name of Glaphyroptera Winnertz, 1863, not of Heer, 1852. Type, Leia fascipennis Meigen. Equals Leia MeIgeN, 1818.

Neoidiotypa Osten Sacken, Cat. Dipt. N. Amer., p. 187, 1878. Change of name for Idiotypa LoEw, 1873, not of FErster, 1856. Type, Idiotypa appendiculata LoEw. Syn., Idiotypa Loew, 1873, preoceupied.

Neoitamus Osten Sacken, Cat. Dipt. N. Amer., p. 82, 1878. Change of name for Itamus Loew, 1849, not of Schmidt-Gerel, 1846. Type, Asilus cyanurus Loew. Syn., Itamus Loew, 1849, preoccupied.

Neolasioptera FeLt, 23d Rep. State Ent. N. Y., p. 330, 1908. 32 species. Type, Lasioptera vitinea FELT, the seventh species, by present designation.

Neolimnophora Schnabl, Zeitschr. Hym. Dipt., vol. 2, p. 94, 1902.1 species. Type, Melanochelia maritima RöDER. Equals Limnophora DesvoIdy, 1830.

Neomochtherus Osten Sacken, Cat. Dipt. N. Amer., p. 82, 1878. Change of name for Mochtherus Loew, 1849, not of Schmidt-Geber, 1846. Type, Asilus pallipes Meigen. Equals Heligmonevra Bigot, 1858.

Neopachygaster Austen, Ent. Monthly Mag., vol. 12, p. 245, 1901. 1 species. Type, Sargus pachygaster Fallen (as Pachygaster meromelas Dufour). Equals Pachygaster Meigex, 1803.

Neopales Coquillett. New name for Pales Desvoidy, 1830, not of Meigen, 1800. Type, Tachina processionex Ratzeburg. Syn., Pales Desvoldy, 1830, preoceupied.

Neophoneus Williston, Psyche, vol. 5, p. 255, 1889. Change of name for Phoneus Macquart, 1838, not of Kaup, 1829. Type, Phoneus servillei Macquart. Syn., Phoneus Macquart, 1838, preoceupied.

Neophyto Townsend, Smiths. Misc. Coll., vol. 51, p. 55, 1908.1 species (as 2). Type, Phyto setosa Coquillett.

Neoplasta Coquillett, Proc. U. S. Nat. Mus., vol. 18, p. 392, 1896. 1 species. Type, Hemerodromia scapularis LoEw.

Neoptera Wulp, Biol. Centr.-Amer., Dipt., vol. 2, p. 165, 1890. 1 species. Type, Eistrophasia bilimekii Brauer and Bergenstamm (as rufa, new species). Equals Qrmia Desvoldy, 1830.

Neorondania Osten Sacken, Cat. Dipt. N. Amer., p. 50, 1878. Change of name for Rondania J NICkE. Syn., Rondania J ENNıcke, 1867, preoccupied.

Neotractocera Townsexd, Trans. Amer. Ent. Soc., vol. 19, p. 105, 1892.1 species. Type, Neotractocera anomala Townsend. Equals Hypertrophocera Townsend, 1891.

Nephrocerus Zetterstedt, Ins. Lapp., p. 578, 1838. 1 species. Type, Nephrocerus lapponicus ZETTERSTEDT.

Nerea Desvoldy, Essai Myod., p. 802, 1830. 3 species. Type, Copromyza limosa Fallen (as riparia, new species), the first species, by present designation. Equals Leptocera OLivier, 1813.

Neria Desvordy, Essai Myod., p. 736, 1830. 9 species. Type, Musca ephippium FAbricius (as inulx, new species), the eighth species, by present designation. Equals Trepidaria MeIGEN, 1800.

Nerina Desvoldy, Essai Myod., p. 557, 1830. 5 species. Type, Nerina albipennis FAILEN, the second species, by present designation. Equals Pegomya Desvoidy, 1830.

Nerius Fabricius, Syst. Antliatorum, p. 264, 1805. 3 species. Type, Nerius pilifer , FABricius, the first species, by present designation.

Neurigona Rondan1, Dipt. Ital. Prodr., vol. 1, p. 142, 1856.1 species. Type, Musca quadrifasciata Fabricius. Syn., Saucropus Loww, 1857; Dactylomyia AldRICH, 1894.

Neuroctena Rondani, Atti Soc. Ital. Sci., vol. 11, p. 56, 1868. 1 species. Type, Dryomyza anilis FALLEN. 
Nevrorta Lioy, Atti Inst. Veneto, ser. 3, vol. 9, p. 910, 1864. 1 species. Type, Musca grisea Fallen. Equals Hylemya Desvoldy, 1830.

$\checkmark \quad$ Nicocles Jenvicke, Neue Exot. Dipt., p. 47, 1867. 1 species. Type, Nicocles analis J «xicke. Syn., Pygostolus Loew, 1866 , preoceupied.

- Nigrasilus Hive, Can. Ent., vol. 40, p. 203, 1908. 1 species. Type, Nigrasilus nitidifacies Hine.

$\checkmark \quad$ Nilea Desvony, Hist. Nat. Dipt., vol. 1, p. 275, 1863. 1 species. Type, Tachina fauna Meigen (as innoxia, new species). Equals Zenilla Desvoidy, 1830.

- Ninguis W Allengrex, Ent. Tijdschr., vol. 2, p. 183, 1881. 3 species. Type, Limnobia virgo ZеттеніsтеDт, the last species, by present designation. Equals Orimarga Osten SACKen, 1869.

$\checkmark$ Nirmomyia Nıтzsch, Germ. Mag. Ent., vol. 3, p. 309, 1818. 1 species. Type, Hippobosca equina Linneus. Equals Hippobosca Linn.eus, 1758.

$\checkmark \quad$ Nitellia Desvoidy, Essai Myod., p. 417, 1830. 2 species. Type, Musca vespillo Fabricius, the first species, by present esignation. Equals Pollenia Destoidy, 1830.

$\checkmark$ Noda Schellenberg, Gen. Mouch. Itipt., pl. 12, fig. 1, 1803. 2 species (without names). Type, Musca aterrim Fabricius, the first species, by present designation. Equals Phora Latreille, 1796.

` Nodutis Meigen, Syst. Beschr. Zweifl. Ins., vol. 2, p. 106, 1820. 1 species. Type, Sylvicolæ melancholia "Härris (as maculatu, new species). Equals Atherix Meigen, 1803.

C Nœza Meigen, Nouv. Class. Mouch., p. 27, 1800.* No species. Hybos Meigen, 1803 , is a change of name. Type, Musca grossipes Linnecs. Syn., Hybos Meigen, 1803; Lactistommia Melander, 1902.

ᄂ Nostima Coqullett, Can. Ent., vol. 32, p. 35, 1900. 1 species. Type, Nostima slossonic Coqulletet.

$\checkmark$ Nothomyia Loew, Berliner Ent. Zeitschr., vol. 13, p. 4, 1869. 2 species. Type, Nothomyia scutellata Loew, the first species, by designation of Brauer, Denkschr. Kais. Akad. Wiss. Wien, vol. 44, 1882, p. 88.

$\checkmark$ Nothosympycnus Wheelel, Proc. Cal. Acad. Sci., vol. 2, p. 51, 1899. 6 species. Type, Nothosympycnus regetus WhenLer, the second species, by present designation.

$\checkmark$ Nothra Westwood, Trans. Ent. Soc. Lond. for 1876, p. 514, 1876. 1 species. Type, Nothra bicolor Westwoon.

$\checkmark$ Notiphila F allen, Spee. Ent. Meth. Exb., p. 22, 1810. No species. In the Kongl. Vet. Acad. Handl. for 1813 , pP. 249 to 255,15 species. Type, Notiphila cinerea Falles, the second species, by designation of Westwoon, Intr., vol. 2, 1840, Synops., p. 153. Syn., Keratocera Desvoidy, 1830.

- Notogramma Loew, Berliner Ent. Zeitschr., vol. 11, p. 289, 1868. 1 species. Type, Musca stigma FABricius (as cimiciformis, new species).

$\checkmark$ Nototricha Coquillett, Class. Mosq. N. Amer., p. 12, 1906.1 species. Type, Cyrlolepteron mediopunctatus THEoвaLD.

$\checkmark \quad$ Noria Desvomy, Essai Myod., p. 210, 1830. 1 species. Type, Tachina melanocephala Meigen (as cylindrica, new species). Equals Phyto Desrordy, 1830.

$\checkmark$ Nowickia Wacht, Wien. Ent. Zeit.; vol. 13, p. 142, 1894. 1 species. Type, Tuchina marklini Zetrenstedt (as regalis Rondan1). Equals Larvævora Meigen, 1800 .

Nuceria Walker, Ins. Saund., Dipt., p. 7, 1850. 5 species. Type, Pangonius longirostris HARDWlckE, the first species, by present designation. Equals Pangonius LATreille, 1802.

$\checkmark$ Numantic Bigot, Anu. Soc. Ent. France for 1854, p. 470, 1854 20 species. Type, Limonia fusca Meigen, the first species, by present designation. Equals Furcomyia Meigen, 1818.

$\checkmark \quad$ Nupharia Desvoiny, Essai Myod., p. 785, 1830. 1 species. Type, IIusca livens Fabricius (as rimularis, new species). Equals Hydromyza Fallen, 1813. 
Nycteribia Latreille, Precis, p. 176, 1796. 1 species. Type, Pediculus vespertilionis Linneus. Syn., Phthiridium Hermann, 1804; Celeripes Montagu, 1815; Stylidia Westwood, 1840; Acrocholidia Kolenati, 1857; Listropoda Kolenati, 1857.

Nyssorhynchus Blancha RD, Compt. Rend. Soc. Biol., p. 795, July 4, $1902 . \quad$ Change of name for Laverania -Theobald, June 16, 1902, not of Grassi and Feletti, 1890. Type, Anopheles argyritarsis Desvoidy. Equals Cellia Theobald, June 16, 1902.

Oblicia Desvoldy, Essai Myod., p. 620, 1830. 1 species. Type, Musca fimetaria Linneus (as testacea, new species). Equals Psila Meigen, 1803.

$\checkmark$ Obolodiplosis Felt, 23d Rep. State Ent. N. Y., p. 410, 1908. 1 species. Type, Cecidomyia orbiculata FeLt.

Ocalea Desvoidy, Hist. Nat. Dipt., vol. 1, p. 8f0, 1863. 1 species. Type, Ocyptera costata Fallen (as heterocera, new species). Equals Wagneria Desvoldy, 1830.

Occemya Desvoldy, Dipt. Env. Paris, Myop., p. 50, 1853.* 2 species. Type, Myopa atra FABricius, the second species, by present designation. Equals Thecophora RONDANI, 1845.

Ochlerotatus Arribalzaga, Rev. Mus. La Plata, vol. 1, p. 385, 1891. 2 species. Type, Ochlerotatus confirmatus ARRIBALZAGA, the second species, by present designation. Syn., Culicada Felt, 1904; Culicelsa Felt, 1904; Ecculex Felt, 1904; Protoculex Felt, 1904; Pseudoculex Dyar, 1905; Pseudohowardina Theobald, 1907; Protomacleaya Theовацd, 1907.

Ochthera Latreille, Hist. Nat. Crust. et Ins., vol. 3, p. 462, 1802. 1 species (as 2). Type, Musca mantis De Geer. Syn., Macrochira Meigen, 1803.

Ochtheroidea Williston, Trans. Ent. Soc. Lond. for 1896, p. 401, 1896. 1 species. Type, Ochtheroidea atra Williston.

Ochtiphila Fallen, Phytom. Ocht. Sveciæ, p. 9, 1823. 3 species. Type, Ochtiphila aridella FALLEN, the second species, by designation of Westwood, Intr., vol. 2, Synops., 1840, p. 151. Equals Chamæmyia Meigen, 1803.

Ocnæa Erichson, Entomographien, p. 155, 1840. 3 species. Type, Ocnæa micans Erichson, the first species, by present designation.

Ocneros Costa, Atti Acad. Sci., vol. 5, pt. 2, p. 102, 1844. 1 species. Type, Musca pulchella Rossi. Equals Palloptera Fallen, 1820.

Ocromyia Lioy, Atti Inst. Veneto, ser. 3, vol. 9, p. 910, 1864. 1 species. Type, Leucophora cinerea Desvoidy (as pallida MacQuart). Equals Leucophora DesvoIDr, 1830.

$\checkmark$ Octacantha Lioy, Atti Inst. Veneto, ser. 3, vol. 9, p. 586, 1864. 2 species. Type, Beris fuscipes MeIgen, the first species, by present designation. Equals Beris LATREILle, 1802.

Octavia Bigot, Ann. Soc. Ent. France for 1854 , p. 474, 1854. 12 species. Type, Erioptera tænionota Meigen, the second species, by present designation. Equals Polymeda Meigen, 1800.

Ocydromia Meigen, Syst. Beschr. Zweifl. Ins., vol. 2, p. 351, 1820. 5 species. Type, Empis glabricula FALLEN, the first species, by designation of Westwood, Intr., vol. 2, Synops., 1840, p. 133.

Ocyptamus Macquart, Hist. Nat., Dipt., vol. 1, p. 554, 1834. 2 species. Type, Baccha fuscipennis $\mathrm{SAY}$ (as fascipennis, new species), the second species, by present designation.

Ocyptera Latreille, Hist. Nat. Crust. et Ins., vol. 14, p. 378, 1805. 3 species. Type, Musca brassicaria FABRIcius, the second species, by designation of CurTis, Brit. Ent., 1837, p. 629. Equals Cylindromyia MeIgeN, 1803.

Ocypterosipha Townsend, Journ. N. Y. Ent. Soc., vol. 2, p. 79, 1894. 1 species. Type, Tachina rlops WALKer (as willistonii, new species). Equals Beskia Brauer and Bergenstamm, 1889.

Proc.N.M.vol.37-10-37 
$\checkmark$ Odinia Desvordy, Essai Myod., p. 648, 1830. 2 species. Type, Milichia maculate Meigen (as trinotata, new species), the first species, by designation of Rondani, Bull. Soc. Ent. Ital., vol. 7,1875 , p. 2. Syn., Alticomerus Rondani, 1856.

$\checkmark$ Odontocera Macquart, Hist. Nat., Dipt., vol. 2, p. 614, 1835. 5 species. Type, Chlorops denticornis PANZER, the first species, by original designation. Not Odontocera Serville, 1833. Equals Cerodontha Rondani, 1861.

$\checkmark$ Odontodiplosis FeLt, 23d Rep. State Ent. N. Y., p. 404, 1908. 1 species. Type, Cecidomyia karnerensis Felt.

Odontomera MAcQuart, Dipt. Exot., vol. 2, pt. 3, p. 372, 1843.1 species. Type, Odontomera ferruginea Macquart. Syn., Cyrtometopa Loew, 1873.

- Odontomyia Meigen, Illiger's Mag., vol. 2, p. 265, 1803. 3 species. Type, Musca hydroleon Linneus, the third species, by designation of Westwoon, Intr., vol. 2, Synops., 1840, p. 130 . Equals Eulalia Meigen, 1800.

Odontonyx RubsaAmen, Berliner Ent. Zeitschr., vol. 39, p. 25, 1894. 3 species. Type, Sciara nigra Wiedemann, the second species, by present designation. Not Odontonyx Stephens, 1828. Equals Phorodonta, new name.

$\checkmark$ Odontopoda Aldrich, 21st Ann. Rep. Geol. Ind., p. 187, 1897. 1 species. Type, Odontopoda sayi A LDRICH.

$\checkmark \quad$ Ecacta Poey, Mem. Hist. Nat. Cuba, vol. 1, p. 236, 1851. 1 species. Type, Ecacta furens Poey. Equals Culicoides Latreille, 1809.

- Edalea Meigen, Syst. Beschr. Zweifl. Ins., vol. 2, p. 355, 1820. 2 species. Type, Empis hybotina Fallen, the first species, by designation of Westwood, Intr., vol. 2, Synops., 1840, p. 133. Syn., Xiphidicera Macquart, 1834.

$\checkmark$ Edaspis Loew, Monogr. Trypet., p. 46, 1862.* 4 species. Type, Trypeta multifasciata Loew, the second species, by present designation. Equals Orellia Desvoidy, 1830.

- Edemagena Latreille, Nouv. Dict. Hist. Nat., vol. 23, p. 272, 1818. 1 species. Type, Estrus tarandi Linn eus.

- Tdemapeza Townsend, Smiths. Misc. Coll., vol. 51, p. 66, 1908. 1 species. Type, Atrophopoda townsendi Williston. Equals Paradidyma Brauer and Bergenstamm, 1891 .

- Edemasoma Townsend, Smiths. Misc. Coll., vol. 51, p. 80, 1908. 1 species. Type, (Edemasoma nuda Townsend.

๔dicarena Loew, Monogr. Dipt. N. Amer., vol. 3, p. 247, 1873.1 species. Type, Trypeta tetanops Loew.

$\checkmark$ Edopa Loew, Berliner Ent. Zeitschr., vol. 11, p. 287, 1868. 1 species. Type, Edopa capito Loew.

Edoparea Loew, Zeitschr. Ent. Breslau, vol. 13, p. 10, 1862. 1 species. Type, Heteromyza buccata Fallen. Equals Heteromyza FAllen, 1820.

Estrophasia Brauer and Bergenstamm, Denkschr. Kais. A kad. Wiss. Wien, vol. 56, p. $145,1889.2$ species. Type, Estrophasia clausa Braver and Bergenstani, the first species, by designation of Townsend, Trans. Amer. Ent. Soc., vol. 19, 1892, p. 133. Equals Ormia Desvoidy, 1830.

$\checkmark$ Estrus Linnwus, Syst. Nat., 10th ed., p. 584, 1758. 5 species. Type, (Estrus oris Linneus, the fifth species, by designation of CurTis, Brit. Ent., 1826, p. 106. Syni, Cephalemyia Latreille, 1818.

Ogcodes Latreille, Precis, p. 154, 1796. No species. In his Hist. Nat. Crust. et Ins., vol. 3, 1802, p. 432, 1 species. Type, Ogcodes zonatus Erichson (as Syrphus gibbosus Fabricius). Syn., Henops Meigen, 1804.

Su Ogcodocera Macquart, Dipt. Exot., vol. 2, pt. 1, p. 83, 1840. 1 species. Type, Mulio leucoprocta Wiedenann (as dimidiata, new species).

Okenia Zetterstedt, Ins. Lapp., p. 734, 1838. 2 species. Type, Cordilura caudata ZetTerstedt, the first species, by Becker, who, in the Berliner Ent. 
Zeitschr., vol. 39,1894 , p. 141, selected the second species as type of a new genus, Bostrichopyga. Not Okenia Levckart, 1826. Equals Okeniella Hendel, 1907.

$\checkmark$ Okeniella Hendel, Wien. Ent. Zeit., vol. 26, p. 98, 1907. Change of name for Okenia Zetterstedt, 1838, not of Leuckart, 1826. Type, Cordilura caudatu ZetterSTEDT. Syn., Okenia ZETTERstedt, 1838, preoccupied.

Olbiogaster Osten Sacken, Biol. Centr.-Amer., Dipt., vol. 1, p. 20, 1886.2 species. Type, Rhyphus treniatus BeLlardi, the second species, by present designation.

Olenochaeta Townsend, Trans. Amer. Ent. Soc., vol. 19, p. 114, 1892.1 species. Type, Distichona varia WulP (as kansensis, new species). Equals Distichona Wulp, 1890.

Olfersia Wiedemann, Auss. Zweifl. Ins., vol. 2, p. 605, 1830. Change of name for Feronia Leach, 1817, not of Latreille, 1817. Type, Feronia spinifera Leach. Syn., Feronia LeACH, 1817, preoccupied.

Oligarces Meinert, Nat. Tidsskr. for 1865 , p. 237, 1865.* 1 species. Type, Oligarces paradoxus MEINERT.

Oligochætus Mıк, Jahresb. k. k. Akad. Gymnas., p. 7, 1878. 5 species. Type, Medetera plumbella Melgen, the first species, by original designation. Equals Medetera Fischer, 1819.

Oligomera Doleschall, Nat. Tijdschr. Ned. Indie, vol. 14, p. 387, 1857. 1 species. Type, Limnobia acrostacta Wiedemann (as javensis, new species). Equals Caloptera Guerin, 1829.

Oligotrophus Latreille, Hist. Nat. Crust. et Ins., vol. 14, p. 288, 1805. 1 species. Type, Tipula juniperina Linn.eus (as "Tipule des galles du genevrier" of De GeER)

Olina Desvordy, Essai Myod., p. 812, 1830. 5 species. Type, Olina hirtipes Desvoiny, the third species, by present designation.

Olivieria Meiges, Syst. Beschr. Zweifl. Ins., vol. 7, p. 266, 1838. 1 species. Type, Tachina longirostris Meigen. Equals Aphria Desvoidy, 1830.

Omalocephala Macquart, Dipt. Exot., vol. 2, pt. 3, p. 231, 1843.1 species. Type, Omalocephala fusca Macquart. Not Omalocephala Spivola, 1839. Equals Tylemyia Giglio-Tos, 1893.

Omegasyrphus Giglio-Tos, Boll. Mus. Zool. Univ. Torino, vol. 6, no. 108, p. 4, 1891. No species. In 1892, 1 species. Type, Microdon coarctatus LoEw.

Ommatius. Wiedemann, Dipt. Exot., p. 213, 1821. 3 species. Type, Asilus marginellus $\mathrm{F}_{\mathrm{ABRICIUS}}$, the first species, by present designation.

Omomyia Coquilletr, Can. Ent., vol. 39, p. 76, 1907. 1 species. Type, Omomyia hirsuta COQUILletT.

Omphrale Meigen, Nouv. Class. Mouch., p. 29, 1800.* No species. Hypselura Meigex, 1803, is a change of name. Type, Musca fenestralis Linneus. Syn., Scenopinus Latreille, 1802; Hypseluta Meigen, 1803; Atrichia Schrank, 1803; Cona Schellenberg, 1803; Astoma Lioy, 1864.

Onesia Desvoldy, Essai Myod., p. 365, 1830. 2 species (as 16). Type, Musca sepulchralis Meigen, (equalling 15 of the supposed species), by designation of RonDANi, Dipt. Ital. Prodr., vol. 1, 1856, p. 90 (as vespillo Fabricius). Syn., Melinda Desvoidy, 1830; Bellardia Desvoidy, 1863; Maravigna Lioy, 1864.

Onodontha Rondani, Dipt. Ital. Prodr., vol. 1, p. 94, 1856. 1 species. Type, Onodonthá penicillata Rondani (as Hydrotra floccosa Macquart). Equals Hydrotæa DesvoIDy, 1830.

Onychogonia Brauer and Bergenstamm, Denkschr. Kais. Akad. Wiss. Wien, vol. 56, p. 100, 1889. 1 species. Type, Gonia flaviceps Zetтerstedt. Equals Salmacia MeIgen, 1800.

Ophelia Desvordy, Essai Myod., p. 120, 1830. 2 species (as 5). Type, Tachina campestris FALLEN (equaling the last 3 supposed species), by present designation. Equals Metopia MeIGen, 1803. 
Ophromyia Williston, Biol. Centr.-Amer., Dipt., vol. 3, p. 55, Dec., 1891. 1 species. Type, Ophromyia nasica Williston. Equals Megametopon Giglo-Tos, September, 1891.

$\checkmark \quad$ Ophthalmomyia Williston, Trans. Ent. Soc. Lond. for 1896, p. 426, 1896. 1 species. Type, Lobioptera lacteipennis Loew. Equals Milichiella Giglio-Tos, 1895.

$\checkmark$ Ophyra Desvoidy, Essai Myod., p. 516, 1830. 3 species (as 4). Type, Anthomyia leucostoma WiedemanN (equaling the first 2 supposed species), by designation of Westwood, Intr., vol. 2, Synops., 1840, p. 142.

$\checkmark$ Oplachantha Rondani, Arch. Zool., vol. 3, p. 87, 1864. 2 species. Type, Beris mexicana Bellardi, the first species, by original designation. Equals Beris Latreille, 1802.

$\checkmark$ Oplogaster Rondani, Dipt. Ital. Prodr., vol. 1, p. 98, 1856. 1 species. Type, Musca mollicula Fallen.

$\checkmark$ Opomyza Fallen, Opom. Sveciæ, p. 10, 1820. 3 species. Type, Musca germinationis Linsaus, the first species, by designation of Westwood, Intr., vol. 2, Synops., 1840, p. 152.

- Oppia Desvordy, Hist. Nat. Dipt., vol. 2, p. 404, 1863. 1 species. Type, Tachina devia Fallex (as ciligera, new species). Equals Brachicoma Rondan1, 1856.

$\checkmark$ Opsebius Costa, Rend. Soc. Borb. Accad. Sci., new ser., vol. 5, p. 20, 1856. 1 species. Type, Opsebius perspicillatus Costa. Syn., Pithogaster Loew, 1857.

$\checkmark$ Opsidia Coquillett, Journ. N. Y. Ent. Soc., vol. 3, p. 102, 1895. 1 species. Type, Opsidia gonioides CoquilletT.

$\checkmark$ Opsiomyia Coquillett, Journ. N. Y. Ent. Soc., vol. 6, p. 162, 1898. 1 species. Type, Opsiomyia palpalis Coquillett.

$\checkmark$ Opsolasia, new genus. Type, Lasiops calvicrura Coquilletт. (This is Lasiops of authors, not of MEIGEN.)

Orbellia Desvordy, Essai Myod., p. 656, 1830. 2 species. Type, Orbellia myopiformis Desvoldy, the first species, by present designation. Equals Heleomyza FALLEN, 1810.

$\checkmark \quad$ Orellia DesvoIdy, Essai Myod., p. 765, 1830. 1 species. Type, Trypeta wiedemanni Melgen (as flavicans, new species). Syn., Goniglossum Rondani, 1856; Edaspis LoEw, 1862.

$\checkmark$ Oreogeton Schiner, Wien. Ent. Monatschr., vol. 4, p. 53, 1860. 1 species. Type, Gloma basalis LoEw.

$\checkmark \quad$ Oreomyza Pokonxy, Wien. Ent. Zeit., vol. 6, p. 50, 1887. 3 species. Type, Oreomyza glacialis Pokonny, the first species, by present designation. Equals Tipula Linneus, 1758.

- Oreothalia Melander, Trans. Amer. Ent. Soc., vol. 28, p. 232, 1902. 1 species. Type, Oreothalia pelops Melander.

Orillia Desvordy, Ann. Soc. Ent. France for 1848, p. 474, 1848. 2 species (as 3). Type, Orillia rectinervis Desvordy, the second species, by present designation. Equals Leskia DesvoIdy, 1830.

$\checkmark$ Orimarga Osten SAcken, Monogr. Dipt. N. Amer., vol. 4, p. 120, 1869. 1 species. Type, Limnobia alpina Zetterstedt. Syn., Ninguis Wallengren, 1881.

Orimargula Mıк, Wien. Ent. Zeit., vol. 2, p. 198, 1883. 2 species. Type, Orimargula alpigena Mrk, the first species, by original designation. Equals Antocha Osten SACKen, 1860.

Orizia Desvoldy, Hist. Nat. Dipt., vol. 2, p. 678, 1863. 2 species (as 6). Type, Musca rudis FABRicius (equaling the first 5 supposed species), by present designation. Equals Pollenia Desvoldr, 1830.

Ormia Desvordy, Essai Myod., p. 428, 1830. 1 species. Type, Ormia punctata Destordy. Syn., Estrophasia Brauer and Bergenstama, 1889; Phasiopteryx Brauer and Bergenstamm, 1889; Cenosoma Wulp, 1890; Neoptera WulP, 1890; Eurestrophasia Townsend, 1892. 
Ormosia Rondani, Dipt. Ital. Prodr., vol. 1, p. 180, 1856.1 species. Type, Erioptera nodulosa Macquart. Syn., Rypholophus Kolenati, 1860; Dasyptera SCHINER, 1863.

$\checkmark$ Ornidia St. FARgeau and Serville, Encycl. Meth., vol. 10, pt. 2, p. 786, 1828. 1 species. Type, Syrphus obesus Fabricius. Equals Volucella Geofrroy, 1762.

[Ornitheza Speiser, Term. Fuzetek, vol. 25, p. 329, 1902. 3 species. Type, Ornithomyia gestroi Rondani, the first species by original designation. Not American.]

Ornithobia Meigen, Syst. Beschr. Zweifl. Ins., vol. 6, p. 229, 1830. 1 species. Type,

Pediculus cervi Linn sus (as pallida, new species). Equals Lipoptena Nitzsch, 1818.

Ornithoctona Speiser, Term. Fuzetek, vol. 25, p. 328, 1902. 10 species. Type, Ornithomyia erythrocephala LEACH, the first species, by original designation.

Ornithodes Coquillett, Proc. Wash. Acad. Sci., vol. 2, p. 400, 1900. 1 species. Type, Ornithodes harrimani Coquilletr.

Ornithoica Rondani, Ann. Mus. Civ. Genova, vol. 12, p. 159, 1878. 1 species. Type, Ornithoica beccariina Rondan!.

Ornithomyia Latreille, Hist. Nat. Crust. et Ins., vol. 3, p. 466, 1802. 1 species. Type, Hippobosca avicularia Linn eus. mut.

Ornithopertha SPEISER, Zeitschr. Hym. Dipt., vol. 1, p. 167, 1902.1 species. Type, Ornithomyia nitens Brgot. tod. 166

Oropeza NeEDham, in 23d Rep. State Ent. N. Y., p. 211, 1908. 1 species. Type, Oropeza americana NEEDHAM.

Orphnephila Haliday, Zool. Journ., vol. 5, p. 350, Sept., 1831. 1 species. Type, Orphnephila devic Haliday. Syn., Thaumalea Ruthe, November, 1831.

Ortalis Falden, Spec. Ent. Meth. Exh., p. 17, 1810. 3 species. Type, Musca vibrans Linnæus, the second species, by designation of Westwood, Intr., vol. 2, Synops., 1840, p. 149. Syn., Seioptera Kirby, 1817; Myodina Desvoldy, 1830.

Orthacheta Becker, Berliner Ent. Zeitschr., vol. 39, p. 101, 1894. 1 species. Type, Cordilura pilosa Zetterstedt.

Orthellia Desvordy, Hist. Nat. Dipt., vol. 2, p. 837, 1863. 3 species (as 4). Type, Musca cornicina FABRICIUs, equalling the first two supposed species, by present designation. Syn., Euphoria Desvoidy, 1863; Pseudopyrellia Grrschner, 1893.

Orthobates WAHLBERG, Öfv. Kongl. Vet. Akad. Förh., vol. 1, p. 110, 1844. 2 species. Type, Hydrophorus jaculus FaLLEN, the first species, by present designation. Equals Medetera Fischer, 1819.

Orthoceratium Schrank, Fauna Boica, vol. 3, p. 151, 1803.* 1 species. Type, Musca lacustris Scopoli. Syn., Liancalus Loew, 1857; Allooneurus Miк, 1878.

Orthocladius Wulp, Tijdschr. Ent., vol. 17, p. 132, 1874. 10 species. Type, Tipula stercoraria De GEER, the first species, by present designation. Syn., Psectrocladius KIEFFer, 1906; Dactylocladius KiefFer, 1906.

Orthoneuromyia Williston, Kansas Univ. Quart., vol. 2, p. 67, 1893.1 species. Type, Orthoneuromyia modesta Williston. Equals Psilocurus Loew, 1874.

Orthonevra Macquart, Rec. Trav. Soc. Sci. Lille for 1829, p. 188, 1829. 1 species. Type, Chrysogaster elegans Meigen. Equals Chrysogaster Meigen, 1800.

Ortochile Latreille, Gen. Crust. Ins., vol. 4, p. 289, 1809. 1 species. Type Ortochile nigroccruleus LATREILLE.

Osca W ALker, Ins. Saund., Dipt., p. 10, 1850. 2 species. Type, Pangonius depressus Macquart, the first species, by present designation. Equals Pangonius Latreille, 1802.

Oscinimorpha Lioy, Atti Inst. Veneto, ser. 3, vol. 9, p. 1126, 1864.1 species. Type, Oscinis obliqua Macquart. Equals Botanobia Lioy, 1864.

Oscinis Latreille, Hist. Nat. Crust. et Ins., vol. 14, p. 383, 1805. 2 species. Type, Musca lineata Fabricius, the first species, by present designation. Equals Titania Meigen, 1800. (Oscinis of authors equals Botanobia Lioy.) 
Oscinisoma Lroy, Atti Inst. Veneto, ser. 3, vol. 9, p. 1125, 1864. 2 species. Type, Chlorops vitripennis MEIGEN, the first species, by present designation. Equals Botanobia Lioy, 1864.

- Osmra Desvoiny, Essai Myod., p. 8t, 1830. 1 species. Type, Osmæa grisea Desvorny. Equals Actia Desvordy, 1830.

$\checkmark$ Ospriocerus Loew, Berliner Ent. Zeitschr., vol. 10, p. 29, 1866. 2 species. Type, Dasypogon zacus WiedemanN (as æacides, new species), the first species, by designation of BAck, Trans. Amer. Ent. Soc., vol. 35, p. 184, 1909.

Ostracocœlia Giglio-Tos, Boll. Mus. Zool. Univ. Torino, vol. 8, no. 158, p. 10, 1893. 1 species. Type, Ostracoccelia mirabilis Giglio-Tos.

Orycephala Macquart, Dipt. Exot., vol. 2, pt. 3, p. 197, 1843. 1 species. Type, Pyrgota undata Wiedemann (as fuscipennis, new species). Equals Pyrgota WiedeMANN, 1830.

$\checkmark \quad$ Oxycera Meigen, Illiger's Mag., vol. 2, p. 265, 1803. 2 species. Type, Musca hypoleon Linnaus (as trilineata FABricius), the second species, by designation of CurTis, Brit. Ent., 1833, p. 441. Equals Hermione Meigen, 1800.

- Oxyrhina Meigen, Syst. Beschr. Zweifl. Ins., vol. 7, p. 366, 1838. Change of name for Trigonometopus MACQUART, 1835, on the false ground that this name is not appropriate. Type, Tetanocera frontalis Meigen. Equals Trigonometopus Macquart, 1835 .

Ozodiceromyia Bigot, Ann. Soc. Ent. France for 1889, p. 321, 1889. 1 species. Type, Ozodiceromyia mexicana Bigot.

$\checkmark$ Pachycephala Liox, Atti Inst. Veneto, ser. 3, vol. 9, p. 13ł3, 1864. 1 species. Type, Tachina gonixformis Meigen. Equals Baumhaueria Meigen, 1838.

$\checkmark$ Pachycerina Macquart, Hist. Nat., Dipt., vol. 2, p. 511, 1835. 1 species. Type, Lauxania seticornis Fallen.

$\checkmark$ Pachygaster Meigen, Illiger's Mag., vol. 2, p. 266, 1803. 1 species. 'Type, Nemotelus ater Panzer. Syn., Tappo Latreille, 1805; Neopachygaster Austen, 1901.

$\checkmark \quad$ Pachymeria Stephens, Syst. Cat. Brit. Ins., vol. 2, p. 262, 1829. 1 species. Type, Empis femorata Fabricius (as ruralis Meigen). Equals Empis Linnees, 1758.

r Pachymerina Macquart, Hist. Nat., Dipt., vol. 1, p. 333, 1834. 4 species. 'Тype, Empis femorata Fabricius, the first species, by designation of Coquillett, Proc. Ent. Sac. Washington, vol. 5, 1903, p. 25t. Equals Empis Linsæus, 1758.

$\checkmark$ Pachyneurella Brues, Trans. Amer. Ent. Soc., vol. 29, p. 382, 1903. 1 species. Type, Phora venata AlDRich. Equals Puliciphora DAHL, 1897.

$\checkmark$ Pachyophthalmus Brauer and Bergenstamm, Denkschr. Kais. Akad. Wiss. Wien, vol. 56 , p. 117, 1889. 1 species. Type, Tachina signata MeIgen. Syn., Sarcomacronychia Tow NSEND, 1892.

$\checkmark$ Pachyrhina Maceunrt, Hist. Nat., Dipt., vol. 1, p. 88, 1834. 8 species. Type, Tipula crocata Linn.eus, the first species, by designation of WEsTwoon, Intr., vol. 2, Synops., 1840, p. 128.

$\checkmark$ Pachystoma Liox, Atti Inst. Veneto, ser. 3, vol. 9, p. 910, 1864. 1 species. Type, Musca flavipennis Falden (as crussirostris MeIgen). Equals Hylemya Desroidy, 1830.

- Pachystomus Latreille, Gen. Crust. Ins., vol. 4, p. 286, 1809. 1 species. Type, Nemotelus cinctus De Geer (as Rhagio syrphoides PAnzer). Equals Erinna Meigen, 1800 .

Pales Desvordy, Essai Myod., p. 154, 1830. 2 species (as 6). Type, Tachina processioner Ratzebirg (as florea, new species), the first species, by designation of Desvorny, Hist. Nat. Dipt., vol. 1, 1863, p. 519 (as strenua Desvordy). Not Pales Meigen, 1800. Equals Neopales, new name.

$\checkmark \quad$ Pallasia Desvoidy, Essai Myod., p. 239, 1830. 1 species (as 2). Type, Murca globosa Fabricius. Equals Cistogaster Latreille, 1829. 
Palloptera Fallen, Ortal. Sveciæ, p. 23, 1820. 4 species. Type, Nusca umbellatarum Fabricius, the first species, by designation of Westwood, Intr., vol. 2, Synops., 1840, p. 150. Syn., Toxonevra MacQuart, 1835; Ocneros Costa, 1844.

Palpibraca Rondani, Ann. Nat. Napoli for 1845, p. 22, 1846** 1 species. Type, Micropalpus muficornis MacquaRT (as hæmorrhoa, new species). Equals Cuphocera Macquart, 1845.

Palpomyia Meigen, Syst. Beschr. Zweifl. Ins., vol. 1, p. 82, 1818.1 species. Type, Ceratopogon faripes Meigen. Equals Helea Meigen, 1800.

Paltostoma Schiner, Verh. Zool.-Bot. Ges. Wien, vol. 16, p. 951, 1866. 1 species. Type, Paltostoma superbiens Schuner.

Palusia Desvordy, Essai Myod., p. 542, 1830. 10 species. Type, Palusia testacea Desvoidy, the eighth species, by designation of Coquillett, Journ. N. Y. Ent. Soc., vol. 9, 1901, p. $139 . \quad$ Equals Cœnosia Meigen, 1826.

Panacris Gerstecker, Linn. Ent., vol. 11, p. 346, 1857. 1 species. Type, Panacris lucida Gerst æCKER.

Paneryma Wulp, Biol. Centr.-Amer., Dipt., vol. 2, p. 387, 1899. 1 species. Type, Paneryma elongata WuLP.

Pangonius -Latreille, Hist. Nat. Crust. et Ins., vol. 3, p. 437, 1802. 3 species. Type, Tabanus proboscideus $\mathrm{F}_{\mathrm{ABR}}$ icius, the third species, by designation of Latreille, Consider. Général., 1810, p. 443 . Syn., Tanyglossa Meigen, 1803; Philoliche WiedeManN, 1828; Nuceria Walker, 1850; Fidena Walker, 1850; Melpia Walker, 1850; Scaptia Walker, 1850; Tacina WALKer, 1850; Phara WALker, 1850; Clanis WALKer, 1850; Osca. WALker, 1850; Plinthina Walker, 1850; Scarphia Walker, 1850; Lilaa Walker, 1850; Diatomineura Rondani, 1864; Erephopsis Rondani, 1864; Corizoneura RoNDANI, 1864.

Panoplites Theobald, Rep. Coll. Mosq. Brit. Mus., p. 5, 1900. 2 species (as 3). Type, Culex titillans Walker (as Triorhynchus trniorhynchus Arribalzaga), the third species, by designation of Nevev-Lemaire, Mem. Soc. Zool. France, vol. 15, 1902, p. 214. Not Panoplites Gould, 1853. Equals Tæniorhynchus ArribalzaGa, 1891.

Pantarbes Osten Sacken, Bull. 3, U. S. Geol.Surv., p. 254, 1877. 1 species. Type, Pantarbes capito Osten Sacken.

Pantophthalmus Thunberg, Mem. Soc. Sei. Goth., vol. 3, p. 7, 1819.* 1 species. Type, Pantophthalmus tabaninus Thunberg. Syn., Acanthomera Wiedemann, 1821; Megalomyia Вigot, 1880.

Panzeria Desvoidy, Essai Myod., p. 68, 1830. 1 species. Type, Tachina rudis Fallen (as lateralis, new species). Equals Ernestia Desvoidy, 1830.

Parabombylius Williston, Journ. N. Y. Ent. Soc., vol. 15, p. 1, 1907. 4 species. Type, Thlipsogaster ater Coquilletr, the first species, by present designation.

Paracantha Coquillett, Journ. N. Y. Ent. Soc., vol. 7, p. 264, 1899. 1 species. Type, Trypeta culta Wiedemann.

Parachæta Coquilletr, Rev. Tach., p. 37, 1897. 1 species. Type, Blepharipeza inermis Bigot.

Paraclius Losw, Monogr. Dipt. N. Amer., vol. 2, p. 97, 1864. 2 species. Type, Pelastoneurus arcuatus Loew, the first species, by present designation.

[Paracompsomyia HougH, Proc. Acad. Nat. Sci. Phila. for 1898, p. 184, 1898. 1 species. Type, Chrysomya regalis Desvordy (as nigripennis, new species). Equals Chrysomya Desvordy, 1830. Not American.]

Paracosmus Osten SACKen, Bull. 3, U. S. Geol. Surv., p. 262, 1877. Change of name for Allocotus Loew, 1872, not of MAYr, 1864. Type, Allocotus edwardsï Loew. Syn., Allocotus Loew, 1872, preoccupied.

Paracrocera Mıк, Wien. Ent. Zeit., vol. 5, p. 276, 1886. 2 species. Type, Acrocera tumida Erichson, the second species, by present designation. Equals Acrocera Meigen, 1803. 
$\checkmark$ Paradejeania Brauer and Bergenstamm, Denkschr. Kais. Akad. Wiss. Wien, vol. 60, p. 147,-1893. 1 species. Type, Dejeania rutilioides J «NNick E.

$\checkmark \quad$ Paradexodes Townsend, Smiths. Misc. Coll., vol. 51, p. 101, 1908. 2 species. Type, Paradexodes aurifrons Townsend, the first species, by original designation. Equals Lydella Desvoidy, 1830.

$\checkmark$ Paradidyma Brauer and Bergenstamm, Denkschr. Kais. Akad. Wiss. Wien, vol. 58, p. 382, 1891. 1 species. Type, Didyma validinervis WulP. Syn., Atrophopoda Townsend, 1891; Ceratomyiella Townsend, 1891; Lachnomma Townsend, 1892; Microchira Brauer and Bergenstamm, 1893; Edemapeza Townsend, 1908; Diaphoropeza Townsend, 1908.

$\checkmark \quad$ Paradiplosis Felt, 23d Rep. State Ent. N. Y., p. 410, 1908. 1 species. Type, Cecidomyia obesa FeLt.

$\checkmark$ Paradmontia Coquilletr, Proc. U. S. Nat. Mus., vol. 25, p. 106, 1902. 1 species. Type, Paradmontia brevis Coquillett.

$\checkmark$ Paraëxorista Brauer and Bergenstamm, Denkschr. Kais. Akad. Wiss. Wien, vol. 56, p. 87, 1889.1 species. Type, Exorista chelonix Rondani. Equals Carcelia DesvoIDr, 1830.

- Parafabricia Brauer and Bergenstamm, Denkschr. Kais. Akad. Wiss. Wien, vol. 61, p. 612, 1894. 2 species. Type, Tachina bicolor W1edemann, the second species, by present designation. Equals Archytas J envicke, 1867.

Parafischeria Townsend, Smiths. Misc. Coll., vol. 51, p. 74, 1908. 1 species. Type, Inemoticus venatoris Coquillett. Equals Demoticus Macquart, 1854.

- Parafrontina Brauer and Bergenstamm, Denkschr. Kais. Akad. Wiss. Wien, vol. 60, p. 115, 1893.1 species. Type, Parafrontina apicalis Brauer and Bergenstamm. Equals Frontina MeIGeN, 1838.

Paragædia Brauer and Bergenstamm, Denkschr. Kais. Akad. Wiss. Wien, vol. 58, p. 350, 1891. 1 species. Type, Blepharipeza cyaneiventris Macquart (as hedemanni, new species).

$\checkmark$ Paragorgopis Giglıo-Tos, Boll. Mus. Zool. Univ. Torino, vol. 8, No. 158, p. 12, 1893. 1 species. Type, Paragorgopis maculata GigL1o-Tos.

$\checkmark$ Paragus Latreille, Hist. Nat. Crust. et Ins., vol. 14, p. 359, 1805. 1 species. Type, Syrphus bicolor Fabricius.

$\checkmark$ Paragymnomma Brauer and Bergenstamm, Denkschr. Kais. Akad. Wiss. Wien, vol. 58, p. 384, 1891. 2 species. Type, Trichophora nigra MacquaRT (as hystrix, new species), the first species, by present designation. Equals Trichophora MacquarT, 1847.

$\checkmark$ Parahypochæta Brauer and Bergenstamm, Denkschr. Kais. Akad. Wiss. Wien, vol. 58, p. 337, 1891. 1 species. Type, Parahypocheta heteroneura Brauer and Bergenstamm.

- Paralimna Loew, Monogr. Dipt. N. Amer., vol. 2, p. 138, 1862. 1 species. Type, Paralimna appendiculata Loew.

- Parallelomma Beck ER, Berliner Ent. Zeitschr., vol. 39, p. 94, 1894. 5 species. Type, Cordilura albipes FALLen, the second species, by original designation. Equals Mosina Desvoldy, 1830.

- Paralucilia Brauer and Bergenstamm, Denkschr. Kais. Akad. Wiss. Wien, vol. 58, p. 391, 1891. 1 species. Type, Musca macellaria Fabricius (as fulvipes Macquart). Paramesia Macquart, Hist. Nat., Dipt., vol. 2, p. 656, 1835. 2 species. Type, Paramesia wesmrlii Macquart, the first species, by designation of Coquillett, Proc. Ent. Soc. Washington, vol. 5, 1903, p. 255. Equals Atalanta Meigen, 1800.

$\checkmark$ Paramintho Brauer and Bergenstamm, Denkschr. Kais. Akad. Wiss. Wien, vol. 58, p. 366, 1891. No species. Wulp, in Biol. Centr.-Amer., Dipt., vol. 2, 1895, p. 266, referred one species to it, as new. Type, Paramintho modulata WulP.

$\checkmark$ Paramyia Williston, Kansas Univ. Quart., vol. 6, p. 1, 1897. 1 species. Type, Paramyia nigra WILLIston. 
Paranaphora Townsend, Smiths. Misc. Coll., vol. 51, p. 72, 1908. 1 species. Type, Ocyptera triquetra Olivier (as diademoides, new species). Equals Ervia Desvoidy, 1830.

$\checkmark$ Paraphyto Coquillett, Journ. N. Y. Ent. Soc., vol. 3, p. 105, 1895. 1 species. Type, Paraphyto chittendeni Coquillett.

Paraplagia Brauer and Bergenstamm, Denkschr. Kais. Akad. Wiss. Wien, vol. 58, p. 354, 1891. 1 species. Type, Tachina trepida Meigen. Equals Voria Desvoidy, 1830.

Paraprosena Brauer and Bergenstamm, Denkschr. Kais. Akad. Wiss. Wien, vol. 56, p. 127, 1889.1 species. Type, Paraprosena waltii Brauer and Bergenstamm.

Pararchytas Brauer and Bergenstamm, Denkschr. Kais. Akad. Wiss. Wien, vol.61, p. 614, 1894. 1 species. Type, Tachina decisa Walker. Equals Larvævora Meigen, 1800.

Pararicia Brauer and Bergenstamm, Denkschr. Kais. Akad. Wiss. Wien, vol. 58, p. 391, 1891. 6 species. Type, Musca pascuorum Meigen, the first species, by designation of Brauer, Verh. Zool.-Bot. Ges. Wien, vol. 43, 1893, p. 508. Equals Muscina Desvoidy, 1830.

Parasetigena Brauer and Bergenstamm, Denkschr. Kais. Akad. Wiss. Wien, vol. 58, p. 339, 1891. 1 species. Type, Chetogena segregata Rondani. Syn., Duponchelia Desvoidy, 1863, preoccupied.

Parasymmictus Bigot, Bull. Soc. Ent. France for 1879, p. 67, 1879. 1 species. Type, Hirmoneura clausa Osten Sacken. Equals Hirmoneura Meigen, 1820.

Parasyntormon Wheeler, Prol. Cal. Acad. Sci., vol. 2, p. 41, 1899. 6 species. Type, Parasyntormon asellus Wheeler, the first species, by present designation.

Parathalassius Mik, Wien. Ent. Zeit., vol. 10, p. 217, 1891. 1 species. Type, Parathalassius blasigii Мıк.

Paratissa Coquillett, Can. Ent., vol. 32, p. 36, 1900. 1 species. Type, Drosophila pollinosa Williston.

Paratropesa Schiner, Verh. Zool.-Bot. Ges. Wien, vol. 16, p. 932, 1866. 1 species. Type, Paratropesa singularis Schiner.

Parepalpus Coquillett, Proc. U. S. Nat. Mus., vol. 25, p. 120, 1902.1 species. Type, Parepalpus favidus Coquillett.

Parephydra Coquillett, Journ. N. Y. Ent. Soc., vol. 10, p. 183, 1902.1 species. Type, Parephydra humilis Coquillett.

Pareudora WAchtL, Wien. Ent. Zeit., vol. 13, p. 141, 1894. 1 species. Type, Tachina præceps Meigen. Equals Larvævora Meigen, 1800.

Parhydrophorus Wheeler, Ent. News, vol. 7, p. 185, 1896. 1 species. Type, Parhydrophorus canescens Wheeler. Equals Aphrozeta Perris, 1847.

Parodinia Coquillett, Journ. N. Y. Ent. Soc., vol. 10, p. 186, 1902. 2 species. Type, Parodinia cinerea Coquillett, the first species, by original designation.

Parœdopa Coquillett, Journ. N. Y. Ent. Soc., vol. 8, p. 22, 1900. 1 species. Type, Parodopa punctigera Coquillett.

Parthenia Desvoidy, Essai Myod., p. 231, 1830. 2 species (as 3). Type, Parthenia boscii Desvordy, the last species, by present designation. Equals Cylindromyia Meigen, 1803.

Parydra Stenhammer, Kongl. Vet. Akad. Handl. for 1843, p. 144, 1844. 2 species. Type, Ephydra aquila FalleN, the second species, by present designation. Equals Napæa DesvoIdy, 1830.

Paykullia Desvoidy, Essai Myod., p. 270, 1830. 1 species (as 3). Type, Paykullia rubricornis Desvoldy. Equals Melanophora MeIgen, 1803.

Peckia Desvordy, Essai Myod., p. 335, 1830. 5 species. Type, Peckia imperialis Desvoidy, the first species, by present designation. Syn., Phrissopodia Macquart, 1835.

Pedicella Bigot, Ann. Soc. Ent. France for 1856, p. 85, 1856. No species. In 1879, 6 species. Type, Macrosargus temuiventris Bigot, the first of the new species, by 


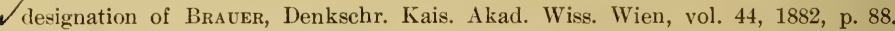
Syn., Macrosargus Bigot, 1879.

$\checkmark$ Pedicia Latreille, Hist. Nat. Crust. et Ins., vol. 4, p. 255, 1809. 1 species. Type, Tipula rivosa Linneus.

$\checkmark \quad$ Pegomya Desvordy, Essai Myod., p. 598, 1830. 6 species. Type, Musca hyoscyami PAnzer, the first species, by designation of Coquillett, Journ. N. Y. Ent. Soc., vol. 9, 1901, p. 140 . Syn., Nerina Desvordy, 1830; Adia Desvoidy, 1830; Phorbia Desvoidy, 1830; Egle Desvoidy, 1830; Phoræa Desvoidy, 1830; Chlorina Desvoidy, 1830; Chortophila MacQuart, 1835; Gymnogaster Lioy, 1864; Botanoplila Lioy, 1864; Trigonostoma Lioy, 1864; Psilometopia Lioy, 1864; Erioischia Lroy, 1864; Stenogaster Lioy, 1864.

$\checkmark \quad$ Pelagomyia Williston, Man. N. Amer. Dipt., p. 48, Aug., 1896. No species. In Trans. Ent. Soc. Lond. for 1896 (Sept.), p. 299, 1 species. Type, Pelagomyiu albitalus Williston.

$\checkmark \quad$ Pelastoneurus Loew, Neue Beitr., vol. 8, p. 36, 1861. 10 species. Type, Pelastoneurus vagans Loew, the fifth new species, by present designation. Syn., Metapelastoneurus ALDRich, 1894.

$\checkmark$ Pelatachina MEADE, Ent. Monthly Mag., vol. 30, p. 109, 1894. Change of name for Hyria Desvoidy, preoccupied. Type, Tachina tibialis Falden. Syn., Hyria DesvoIDY, 1863, preoccupied.

$\checkmark$ Pelecocera Meigen, Syst. Beschr. Zweifl. Ins., vol. 3, p. 340, 1822. 1 species. Type, Pelecocera tricincta Meigen. Syn., Euceratomyia Williston, 1884.

$\checkmark \quad$ Peleteria Desvordy, Essai Myod., p. 39, 1830. 4 species. Type, Peleteria abdominalis Desvoidy, the last species, by present designation. Syn., Faurella Desvordy, 1830; Echinogaster Lioy, 1864; Sphyricera Lioy, 1864; Sphyromyia Brgot, 1883; Tetrachata Brauer and Bergenstamm, 1894; Chrtopeleteria Miк, 1894; Popelia Bezzi, 1894; Tessarochxta BEZzI, 1906.

$\checkmark$ Pelethophila Hagenbach, Sym. Faunæ Inst. Helvetiæ, p. 48, 1822.* 1 species. Type, Musca fimetaria Linneus (as flava Schellenberg). Equals Psila Migigen, 1803.

- Pelina Haliday, Ann. Nat. Hist., vol. 3, p. 407, 1839. 1 species. Type, Notiphila renea Fallen. Syn., Telmatobia Stenhammer, 1844.

$\checkmark$ Pelomyia Williston, N. Amer. Fauna, No. 7, p. 256, 1893. 1 species. Type, Pelomyia occidentalis Williston.

2 Pelopia Meigen, Nouv. Class. Mouch., p. 18, 1800* No species. Tanypus Meigen, 1803, is a change of name. Type, Tipula cincta Fabricius. Syn., Tanypus Meigen, 1803; Protenthes Johannsen, 1907.

- Pelorempis Johannsen, Bull. 295, N. Y. State Mus., p. 402, Aug. 11, 1903.1 species. Type, Eucorethra underwoodi UNDERwood (as americana, new species). Equals Eucorethra Underwood, August 7, 1903.

$\checkmark$ Peloropeodes Wheeler, Psyche, vol. 5, p. 373, 1890. 1 species. Type, Peloropeodes salax WHEELER.

$\checkmark$ Penicillidia Kolenat, Hor. Soc. Ent. Ross., vol. 2, p. 69, 1863. 2 species (as 3). Type, Nycteribia westwoodi Guerin-Meneville (as dufourii Westwood), the second species, by present designation.

$\checkmark$ Pennapoda Townsend, Ann. Mag. Nat. Hist., vol. 20, p. 282, 1897. 1 species. Type, Trichiopoda phasiana Townsend. Equals Trichiopoda Latreille, 1829.

Pentacricia Ste1n, Berliner Ent. Zeitschr., vol. 42, p. 249, 1898. 1 species. Type, Pentacricia aldrichii STEIN.

$\checkmark$ Penthesilia Meigen, Nouv. Class. Mouch., p. 35, 1800.* No species. Criorhina Meigen, 1822, is a change of name. Type, Syrplus asilicus Fallen. Syn., Criorhina Meigen, 1822; Somula Maceuart, 1847; Eriophora Philippl, 1865; Brachymyia WiLListon, 1882; Eurhinomallota Bigot, 1882; Cynorhina Williston, 1886.

$\mathcal{L}$ Penthoptera Schiner, Wien. Ent. Monatschr., vol. 7, p. 220, 1863. 1 species. Type, Tipula chirothecata ScopoLI. 
Penthosia WulP, Tijdschr. Ent., vol. 35, p. 189, 1892. 1 species. Type, Scopolia satanica Bigot.

Peratochetus Rondani, Dipt. Ital. Prodr., vol. 1, p. 119, 1856. 1 species. Type, Heteromyza flava Meigen (as lutescens, new species). Equals Clusia Haliday, 1838. $\checkmark$ Peribra Desvoidy, Hist. Nat. Dipt., vol. 1, p. 720, 1863. 1 species (as 3). Type, Peribra apicalis Desvordy. Equals Actia Desvordy, 1830.

Pericoma Halid AY, in Walker's Ins. Britt., Dipt., vol. 3, p. 256, 1856. 11 species. Type, Trichoptera trifasciata MeIgen, the seventh species, by present designation.

Perithinus Halidar, Zool. Journ., vol. 5, p. 353, 1831. 4 species. Type, Porphyrops riparius Meigen, the first species, by designation of Westwood, Intr., vol. 2, Synops., 1840, p. 134. Syn., Hydrochus Fallen, 1823, preoceupied; Anglearia Carlier, 1835.

Peronyma LoEw, Monogr. Dipt. N. Amer., vol. 3, p. 250, 1873.1 species. Type, Trypeta sarcinata Loew.

Perrisia Rondani, Nuovi Ann. Sci. Nat. (Bologna), ser. 2, vol. 6, p. 371, 1846. 1 species. Type, Cecidomyia urticæ Perris. Equals Dasineura Rondani, 1840.

Petalophora Macquart, Hist. Nat., Dipt., vol. 2, p. 454, 1835. 1 species. Type, Trypeta capitala Wiedemann. Equals Ceratitis Mac Leay, 1829.

Petaurista Meigen, Nouv. Class. Mouch., p. 15, 1800* No species. Trichocera MeIgen, 1803, is a change of name. Type, Tipula hiemalis De Geer. Syn., Trichocera Meigen, 1803.

$\checkmark$ Peteina Meigen, Syst. Beschr. Zweifl. Ins., vol. 7, p. 214, 1838. 1 species. Type, Musca erinacea FABricius.

Petinops Brauer and Bergenstamm, Denkschr. Kais. Akad. Wiss. Wien, vol. 58, p. 356, 1891. 1 species. Type, Petinops schnablii Brauer and Bergenstamm. Equals Wagneria DesvoIdy, 1830.

Phalacrocera Schiner, Wien. Ent. Monatschr., vol. 7, p. 224, 1863. 1 species. Type, Tipula replicata Linnweus (as nudicomis SchummeL).

Phalacromya Rondani, Studi Ent., vol. 1, p. 67, 1848. 2 species. Type, Phalacromya submetallica Rondani, the first species, by present designation. Syn., Glaurotyicha Tномson, 1868.

Phalæenula Meigen, Nouv. Class. Mouch., p. 18, 1800** No species. Trichoptera Meigen, 1803, is a change of name. Type, Trichoptera ocellatis Meigen. Equals Psychoda Latreille, 1796.

Phalangus Meigen, Syst. Beschr. Zweifl. Ins., vol. 3, p. 253, 1822.1 species. Type, Mulio virens Fabricius. Equals Pipiza Fallen, 1810.

Phanigaster Lioy, Atti Inst. Veneto, ser. 3, vol. 10, p. 61, 1864. 1 species. Type, Musca helluo Fabricius. Equals Eliozeta Rondani, 1856.

Phantasma Desvoidy, Essai Myod., p. 739, 1830. 2 species. Type, Musca corrigiolata Linnseus (as filiformis $\mathrm{F}_{\mathrm{ABRI}} \mathrm{CIUS}$ ), the first species, by present designation. Equals Tylos Meigen, 1800.

Phaonia Desvoidy, Essai Myod., p. 482, 1830. 5 species. Type, Musca erratica FALLEN (as viarum, new species), the first species, by designation of Coquillett, Journ. N. Y. Ent. Soc., vol. 9, 1901, p. 140. Syn., Fallæa DesvoIdy, 1830; Trennia Desvoidy, 1830; Euphemia Desvoidy, 1830; Rohrella Desvoidy, 1830; Quadrula Pandelle, 1898.

Phara WAlker, Ins. Saund., Dipt., p. 9, 1850. 18 species. Type, Pangonius melanopyga Wiedemans, the first species, by present designation. Equals Pangonius Latreille, 1802.

Phasia Latreille, Hist. Nat. Crust. et Ins., vol. 14, p. 379, 1805. 1 species. Type, Syrphus hemipterus Fabricius (as Thereva coleoptrata FABricius). Syn., Alophora Desvoidy, 1830; Hyalomya Desvoidy, 1830; Phorantha Rondan1, 1862.

Phasioclista Townsend, Trans. Amer. Ent. Soc., vol. 18, p. 369, 1891.1 species. Type, Clytia atra Desvoidy (as metallica, new species). Equals Myiophasia Brauer and Bergenstamm, 1891. 
$\checkmark$ Phasiops Coquillett, Journ. N. Y. Ent. Soc., vol. 7, p. 219, 1899. 1 species. Type, Phasiops flava CoquilletT.

$\checkmark$ Phasiopteryx Braver and Bergenstamm, Denkschr. Kais. Akad. Wiss. Wien, vol. 56, p. 146, 1889. 2 species. Type, Phasiopteryx bilimekii Brauer and Bergenstamu, the first species, by present designation. Equals Ormia Desvordy, 1830.

$\checkmark$ Pheneus Walker, Ins. Saund., Dipt., p. 155, 1851. 1 species. Type, Pheneus tibialis Walker. Syn., Arthrostylum Williston, 1895.

$\checkmark \quad$ Phenicia Desvordy, Hist. Nat. Dipt., vol. 2, p. 750, 1863. 1 species (as 87). Type, Musca sericata Meigen. Equals Lucilia Desvordy, 1830.

$\checkmark$ Pherbina Desvoidy, Essai Myod., p. 687, 1830. 11 species. Type, Musca coryleti Scopoli (as Tetanocera reticulata DumeriL), the third species, by present designation. Equals Tetanocera Latreille, 1805.

$\checkmark$ Pherecida Desvoldy, Hist. Nat. Dipt., vol. 1, p. 1118, 1863. 1 species (as 2). Type, Tachina grisea Fallen. Equals Macquartia Desvordy, 1830.

$\checkmark$ Philia Meigen, Nouv. Class. Mouch., p. 20, 1800.* No species. Dilophus Meigen, 1803, is a change of name. Type, Tipula febrilis Linnæus. Syn., Dilophus Meigen, 1803.

$\checkmark$ Philinta Desvoldy, Essai Myod., p. 568, 1830. 3 species (as 4). Type, Musca canicularis Linveus, the first species, by designation of Coquillett, Journ. N. Y. Ent. Soc., vol. 9, 1901, p. 140. Equals Fannia Desvordy, 1830.

$\checkmark \quad$ Philodendria Rondani, Dipt. Ital. Prodr., vol. 1, p. 136, 1856. 1 species. Type, Musca aterrima Fabricius. Equals Phora Latreille, 1796.

$\checkmark$ Philoliche Wiedemann, Auss. Zweifl. Ins., vol. 1, p. 95, 1828. 14 species. Type, Tabanus rostratus Linn.eus, the second species, by present designation. Equals Pangonius Latreille, 1802.

$\checkmark$ Philonicus Loew,.Linn. Ent., vol. 4, p.144, 1849. 1 species. Type, Philonicus albiceps Meigen.

$\checkmark \quad$ Philophylla Rondaxı, Bull. Soc. Ent. Ital., vol. 2, p. 9, 1870. 1 species. Type, Musra crsio Harris. Equals Acidia Desvoidy, 1830.

2 Philopota Wiedemann, Auss. Zweifl., Ins. vol. 2, p. 17, 1830. 1 species. Type, Philopota conica Wiedemann.

$\checkmark$ Philorus Kellogg, Proc. Cal. Acad. Sci., ser. 3, vol. 3, p. 199, 1903. 3 species. Type, Blepharicera yosemite Osten SACKen, the second species, by present designation.

$\checkmark$ Philygria Stenhammar, Kongl. Vet. Akad. Handl. for 1843, p. 154, 1844. 3 species. Type, Notiphila flavipes $\mathrm{F}_{\text {Allex, }}$ the second species, by present designation.

$\checkmark$ Pholeomyia Bilimek, Verh. Zool.-Bot. Ges. Wien, vol. 17, p. 903, 1867. 1 species. Type, Pholeomyia leucozona BiLiмek.

$\checkmark$ Phoneus Macquart, Dipt. Exot., vol. 1, pt. 2, p. 79, 1838. 1 species. Type, Phoneus servillei Macquart. Not Phoneus Kaup, 1829. Equals Neophoneus WrLListon, 1889.

Phoneutisca Loew, Berliner Ent. Zeitschr., vol. 7, p. 19, 1863. 1 species. Type, Tachydromia maculipennis W ALKER (as bimaculata, new species.)

$\checkmark$ Phoniomyia Theobald, Monogr. Culic., vol. 3, p. 311, 1903. 2 species. Type, Wyeomyia longirostris ThеоваLd, the first species, by designation of Dyar, Proc. Ent. Soc. Washington, vol. 7, 1905, p. 49.

Phora Latreille, Précis, p. 169, 1796. No species. In his Hist. Nat. Crust. et Ins., vol. 3, 1802, p. 464, 1 species. Type, Musca aterrima FABricius. Syn., Trineura Meigen, 1803; Noda Schellenberg, 1803; Philodendria Rondani, 1856. (Phora of authors equals Anevrina Lıy.)

$\checkmark \quad$ Phorra Desvoidy, Essai Myod., p. 600, 1830. 4 species. Type, Phoræa flavescens Desvordy, the first species, by present designation. Equals Pegomya Desvordy, 1830. 
Phorantha Rondani, Dipt. Ital. Prodr., vol. 5, p. 21, 1862. 1 species. Type, Conops subcoleoptrata Linnæus (as musciformis, new species). Equals Phasia LATREILLE, 1805.

Phorbia Desvoidy, Essai Myod., p. 559, 1830. 5 species. Type, Phorbia musca Desvordy, the first species, by present designation. Equals Pegomya Desvoidy, 1830.

Phorella Desvoidy, Essai Myod., p. 362, 1830. 2 species (as 6). Type, Musca carnaria Linnæus (as arvensis, new species), the first species, by designation of Desvoidy, Hist. Nat. Dipt., vol. 2, 1863, p. 530. Equals Sarcophaga Meigen, 1826.

Phoricheta Rondani, Dipt. Ital. Prodr., vol. 4, p. 8, 1861. Change of name for Scopolia Desvoidy, 1830, not of HüBner, 1816. Type, Ocyptera costata Fallen. Equals Wagneria Desvoldy, 1830.

Phormia Desvoidy, Essai Myod., p. 465, 1830. 3 species (as 8). Type, Musca regina Meigen, the second species, by designation of Desvoidy, Bull. Soc. Ent. France for 1849, p. 5. Syn., Protophormia Townsend, 1908.

Phorocera Desvoldy, Essai Myod., p. 131, 1830. 4 species (as 22). Type, Tachina assimilis FALLEN (equaling the supposed species 1, 2, 3, 5, and 17 to 20 ), by designation of Desvordy, Hist. Nat. Dipt., vol. 1, 1863, p. 509. Syn., Salia Desvordy, 1830; Chetogena Rondani, 1856; Spoggosia Rondani, 1859; Eggeria Schiner, 1861; Euphorocera TownsEnd, 1892.

Phoroctenia, new genus. Type, Ctenophora angustipennis Loww. (This is Ctenophora of authors, not of MEIGEN.)

Phorodonta, new name. For Odontonyx Rubsadmen, 1894, not of Stephens, 1828. Type, Sciara nigra Wiedemann. Syn., Odontonyx Rubsaamen, 1894, preoccupied.

Phorosia Desvoidy, Dipt. Env. Paris, Myop , p. 29, 1853.* 1 species. Type, Conops testacea Linnaus. Equals Stomoxoides Sch

Phorostoma Desvoidy, Essai Myod. p. 326, 1830. 1 species. Type, Musca ferina Fallen (as subrotunda, new species). Syn., Myocera Desvoidy, 1830; Amyclra Desvoidy, 1863; Rhamphinina Bigot, 1885; Clinoneura Brauer and Bergenstamm, 1889; Ptilodexia Brauer and Bergenstamm, 1889.

Phortica Schiner, Wien. Ent. Monatschr., vol. 6, p. 433, December, 1862.1 species. Type, Drosophila variegata Fallen. Equals Amiota Loew, May, 1862.

Phosococephala Townsend, Smiths. Misc. Coll., vol. 51, p. 69, 1908. 1 species. Type, Phosococephala metallica Townsend.

Phrissopodia Macquart, Hist. Nat., Dipt., vol. 2, p. 222, 1835. 2 species. Type, Peckia imperialis Desvordy, the first species, by original designation. Equals Peckia Desvoidy, 1830.

$\checkmark$ Phrissopolia Townsend, Smiths. Misc. Coll., vol. 51, p. 93, 1908. 1 species (as 2). Type, Prospherysa crebra Wulp. Equals Chætogædia Brauer and Bergenstamm, 1891. Phronia Winnertz, Verh. Zool.-Bot. Ges. Wien, vol. 13, p. 857, 1863.28 species. Type, Phronia rustica Winnertz, the 22d species, by designation of Johannsen, Gen. Ins., Dipt., Mycet., 1909, p. 96.

Phryne Meigen, Nouv. Class. Mouch., p. 16, 1800* No species. Anisopus Meigen, 1803 , is a change of name. Type, Tipula fuscata Fabricius. Equals Sylvicolæ HARRIS, 1776.

Phryxe Desvoidy, Essai Myod., p. 158, 1830. 8 species (as 36). Type, Tachina vulgaris FALLeN (equaling 22 of the supposed species), by designation of Desvoidy, Hist. Nat. Dipt., vol. 1, 1863, p. 329. Syn., Blepharidea Rondani, 1856; Hemithæa Desvoidy, 1863; Erinia Desvordy, 1863; Blumia, Desvoidy, 1863; Eurigastrina Lioy, 1864; Anoxycampta Bigot, 1880; Ceratoch $x$ ta Brauer and Bergenstam m, 1889; Pseudophorocera Brauer and Bergenstamm, 1889; Pseudoperichrta Brauer and Bergenstamm, 1889; Blepharidopsis Brauer and Bergenstamm, 1891; Catachrta Brauer and BERGENSTAMM, 1891. 
- Phthinia Winnertz, Verh. Zool.-Bot. Ges. Wien, vol. 13, p. 779, 1863. 3 species. Type, Phthinia humilis Winnertz, the first species, by designation of JoHannsen, Gen. Ins., Dipt., Mycet., 1909, p. 83.

$\checkmark$ Phthiria Meigen, Illiger's Mag., vol. 2, p. 268, 1803. 1 species. Type, Bombylius pulicarius Mikax. Syn., Cyclorliynchus Macquart, 1840; Pxcilognathus Jennicke, 1867.

$\checkmark$ Phthiridium Hermann, Mem. Apter., pp. 12 and 15, 1804. 2 species. Type, Phthiridium biarticulatum Hermans, the second species, by present designation. Equals Nycteribia LATREILLE, 1796.

$\checkmark \quad$ Phylarchus Aldrich, Biol. Centr.-Amer., Dipt., vol. 1, p. 342, 1901.1 species. Type, Phylarchus tripartitus ALDRich

- Phylidorea Вigot, Ann. Soc. Ent. France for 1854, p. 456, 1854. 19 species. Type, Limnobia ferruginea Meigen, the ninth species, by present designation. Syn., Pilarid Sintensis, 1889; Lasiomastix Ostex Sackex, 1860; Prionolabis Ostex Sackex, 1860; Dactylolabis Osten SAcken, 1860; Dicranophragma Osten SACken, 1860.

Phyllodromia Zettenstedt, Isis von Oken for 1837 , p. 31, 1837. 3 species. Ty pe, Empis melanocephala Fabricius, the first species, by designation of RoNDAxi, Dipt. Ital. Prodr., vol. 1, 1856, p. 148. Equals Hemerodromia MeIgex, 1822.

$\checkmark \quad$ Phyllogaster Ste1n, Berliner Ent. Zeitschr., vol. 42, p. 256, 1898. 1 species. Type, Phyllogaster cordyluroides STEIN.

$\checkmark$ Phyllolabis Osten Sacken, Bull. 3, U. S. Geol. Surv., p. 202, 1877. 2 species. Type, Phyllolabis claviger Osten SACKen, the first species, by present designation.

$\checkmark$ Phyllomydas B1got, Bull. Soc. Ent. France for 1880 , p. 46, 1880.1 species. Type, Phyllomydas phyllocerus Bigot. Equals Mydas Fabricius, 1794.

$\checkmark$ Phyllomyza Fallen, Spec. Ent. Meth. Exh., p. 20, 1810. No species. In his Phytom. et Ocht. Sveciæ, 1823, p. 8, 1 species. Type, Phyllomyza securicornis FALLEN.

$\checkmark \quad$ Phyllophaga Rondani, Dipt. Ital. Prodr., vol. 1, p. 199, 1856. 1 species. Type, Cecidomyia fusca Meigen. Equals Asphondylia Loew, 1850.

$\checkmark \quad$ Phyllophila Lıoy, Atti Inst. Veneto, ser. 3, vol. 9, p. 1313, 1864. 1 species. Type, Agromyza pallipes Macquart. Equals Agromyza Fallen, 1810.

$\checkmark$ Physecrania Bıgot, Ann. Soc. Ent. France for 1859, p. 123, 1859. 1 species. Type, Physecrania obscura Bigot. Equals Caloptera Greniv, 1829.

$\checkmark$ Physegenua Macquart,. Dipt. Exot., Suppl. 3, p. 60, 1848. 1 species. Type, Physegenua vittata Macquart.

$\checkmark \quad$ Physiphora Falden, Spec. Ent. Meth. Exh., p. 11, 1810. No species. In his Scen. et Con. Sveciæ, 1817, p. 3, 1 species. Type, Musca demandata FAbricius (as splendida, new species). Syn., Chrysomyza Fallen, 1817; Ulidia Meigen, 1826; Chloria Schiner, 1862.

$\checkmark$ Physocephala Schiner, Wien. Ent. Monatschr., vol. 5, p. 137, 1861. 1 species. Type, Conops rufipes FABRICius.

Phyto Desvoldy, Essai Myod., p. 218, 1830. 1 species (as 4). Type, Tachina melanocephala Meigen. Syn., Novia Desvoldy, 1830; Savia Rondaxi, 1861; Kockia Desvoldy, 1863.

Phytobia Lroy, Atti Inst. Veneto, ser. 3, vol. 9, p. 1313, 1864. 1 species. Type, Agromyza errans Meigex. Equals Agromyza Fallen, 1810.

$\checkmark$ Phytomyza Fallen, Spec. Ent. Meth. Exh., p. 21, 1810. 1 species. Type, Phytomyza flaveola Fallen. Syn., Chiromatomyia Hardy, 1849.

$\checkmark$ Phytophaga Rondani, Sopra Nuov. Gen. Ins. Dipt., p. 12, 1840** 1 species. Type, Cecidomyia destructor $\mathrm{S}_{\mathrm{AY}}$ (as cerealis, new species). Syn., Mayetia Kieffer, 1896.

$\checkmark \quad$ Pialoidea Westwoon, Trans. Ent. Soc. Lond. for 1876, p. 514, 1876.1 species. Type, Cyrtus magnus WALKER. 
Picconia Desvordy, Hist. Nat. Dipt., vol. 2, p. 33, 1863. 1 species. Type, Tachina nigripes Fallen (as bipartita, new species). Equals Lydella Desvordy, 1830.

Pictinia Desvoldy, Dipt. Env. Paris, Myop., p. 15, 1853.* 1 species. Type, Pictinia fulvipalpis Desvoidy. Equals Stomoxoides SCH aFFER, 1766.

Pierretia Desvoidy, Hist. Nat. Dipt., vol. 2, p. 422, 1863.14 supposed species. Type, Sarcophaga nigriventris Meigen, equaling supposed species 2, 3, 4, 5, 6, and 12, by present designation. Equals Sarcophaga Meigen, 1826.

Pilaria Sintensis, Sitzb. Nat.-Ges. Dorpat, vol. 8, p. 398, 1889. 2 species. Type, Limnobia pilicornis ZETTERstedT, the second species, by present designation. Equals Phylidorea Bigot, 1854.

$\checkmark$ Piophila Fallen, Spec. Ent. Meth. Exh., p. 20, 1810. 1 species. Type, Musca casei Linn eus. Syn., Tyrophaga KIRby, 1817; Stearibia Lroy, 1864.

Pipiza Fallen, Spec. Ent. Meth. Exh., p. 11, 1810. No species. In his Syrphici Sveciæ, 1817, pp. 58 to 61,8 species. Type, Musca noctiluca Linnded, the second species, by designation of Curtis, Brit. Ent., 1837, p. 669. Syn., Phalangus Meigen, 1822; Heringia Rondani, 1856; Pipizella Rondani, 1856.

$\checkmark$ Pipizella Rondani, Dipt. Ital. Prodr., vol. 1, p. 54, 1856. 1 species. Type, Mulio virens Fabricius. Equals Pipiza Fallen, 1810.

Pipunculus, Latreille, Hist. Nat. Crust. et Ins., vol. 3, p. 463, 1802. 1 species. Type, Pipunculus campestris Latreille. Equals Dorilas Meigen, 1800.

Pissemya Desvordy, Ann. Soc. Ent. France for 1851, p. 318, 1851. 1 species. Type, Gonia fasciata Wiedemann (as atra Meigen). Equals Spallanzania Desvoidy, 1830.

Pithogaster Loew, Wien. Ent. Monatschr., vol. 1, p. 33, 1857. 1 species. Type, Pithogaster inflatus Loew. Equals Opsebius Costa, 1856.

Pityocera Giglio-Tos, Boll. Mus. Zool. Univ. Torino, vol. 11, No. 224, p. 3, 1896. 1 species. Type, Pityocera festro G̀iglio-Tos.

Plagia Meigen, Syst. Beschr. Zweifl. Ins., vol. 7, p. 201, 1838. 6 species. Type, Tachina ruralis FALLEN (as verticalis MEIGEN), the second species, by designation of Rondani, Dipt. Ital. Prodr., vol. 1, 1856, p. 69. Equals Voria Desvoidy, 1830. (Plagia of authors equals Siphoplagia Townsend.)

Plagiocera Macquart, Dipt. Exot., vol. 2, pt. 2, p. 59, 1842. 2 species. Type, Milesia acuta FABRicius (as cruciger IVIEDEMANN), the first species, by present designation. Not Plagiocera Klug, 1834. Equals Meromacrus Rondani, 1848.

Plagiomima Brauer and Bergenstamm, Denkschr. Kais. Akad. Wiss. Wien, vol. 58, p. 384,1891 . 1 species. Type, Plagiomima disparata Brauer and Bergenstamm.

Plagioneurus Loew, Wien. Ent. Monatschr, vol. 1, p. 43, 1857. 1 species. Type, Plagionerurus univittatus LoEw.

Plagiotoma Loew, Monogr. Dipt. N. Amer., vol. 3, p. 252, 1873. 3species. Type, Trypeta obliqua $\mathrm{SAx}$, the second species, by present designation. Not Plagiotoma Claparède and Lachimann, 1858. Equals Tomoplagia, new name.

Plagiprospherysa Townsend, Trans. Amer. Ent. Soc., vol. 19, p. 113, 1892. 2 species. Type, Prospherysa parvipalpis WulP (as valida, new species), the first species, by original designation.

Planes Rondani, Arch. Zool., vol. 3, p. 9, 1864. 1 species. Type, Xylota vagans Wiedemann. Equals Syritta St. Fargeau and Serville, 1828.

Planetolestes Arribalzaga, Anal. Soc. Cient. Argentina, vol. 8, p. 145, 1879.* 1 species. Type, Laphria coarctata Perty. Equals Blepharepium Rondani, 1848.

Platophryma Williston, Trans. Ent. Soc. Lond. for 1896, p. 426, 1896. 1 species. Type, Platophryma nigra Williston.

Platycheirus St. Fargeau and Serville, Encycl. Meth., vol. 10, pt. 2, p. 513, 1828. 6 species. Type, Syrphus scutatus MeIGeN, the first species, by designation of Westrwood, Intr., vol. 2, Synops., 1840, p. 137, 
$\checkmark$ Platychira Rondani, Dipt. Ital. Prodr., vol. 1, p. 64, 1856. 1 species. Type, Musca puparum Fabricius. Equals Meriania Desvolivy, 1830.

Platyenema ZetTenstedt, Ins. Lapp., p. 334, 1838. 1 species. Type, Empis pulicaria Fallen. Equals Atelestus Walker, 1837.

Platycœnosia Strobl, Wien. Ent. Zeit., vol. 13, p. 72, 1894. 1 species. Type, Platycœnosia mikii Strobl. Syn., Choristomma Stein, 1895.

Platynochætus Wiedemann, Auss. Zweifl. Ins., vol. 2, p. 147, 1830.1 species. Type, Syrphus setosus Fabricius.

$\checkmark \quad$ Platypalpus Macquart, Ins. Dipt. Nord France, Separata, p. 92, 1827. 18 species. Type, Musca cursitans Fabricius, the fourteenth species, by designation of Westwood, Intr., vol. 2, Synops., 1840, p. 132.

$\checkmark \quad$ Platupeza Meigen, Illiger's Mag., vol. 2, p. 272, 1803 . No species. In his Klass. Beschr. Zweitr. Ins., 1804, p. 310, 3 species. Type, Platypeza fasciata Meiges, the first species, by designation of Rondani, Dipt. Ital.- Prodr., vol. 1, 1856, p. 139. Equals Clythia Meigen, 1800.

$\checkmark \quad$ Platyptera Meigen, Illiger's Mag., vol. 2, p. 269, 1803. 2 species. Type, Empis platyptera PAnzer, the second species, by tautonymy. Equals Dionnæa Meigex, 1800.

Platypterygia Stephens, Syst. Cat. Brit. Ins., vol. 2, p. 263, 1829. 1 species. Type, Empis borealis Linneus. Equals Empis Linnæus, 1758.

$\checkmark \quad$ Platystoma Meigen, Illiger's Mag., vol. 2, p. 277, 1803. 1 species. Type, Musca seminationis Linn eus. Syn., Hesyquillia Desvoidy, 1830; Megaloglossa Rondaxi, 1869.

Platmura Meigex, Illiger's Mag., vol. 2, p. 264, 1803: No species. In his Klass. Beschr. Zweifl. Ins., 1804, pp. 101, 102, 5 species. Type, Platyura fasciatu Meigen, the second species, by designation of ZtтTerstedt, Dipt. Scand., vol. 10, 1851, p. 4077. Equals Zelmira Meigen, 1800.

Plaxemya Desvoidy, Essai Myod., p. 392, 1830. 1 species. Type, M sca vitripennis Meigen (as sugillatrix, new species). Equals Musca Linneus, 1758.

Plecia Wiedemans, Auss. Zweifl. Ins., vol. 1, p. 72, 1828. 6 species. Type, Hirtea fulvicollis Fabricius, the second species, by designation of Blanchard, Hist. Nat. Ins., vol. 3, 1840, p. 576. Syn., Rhinoplecia Bellardi, 1859.

Plectops Coquilletr, Rev. Tach., p. 57, 1897. 1 species. Type, Plectops melissopodis CoquilletT.

$\checkmark \quad$ Plectromyia Osten SAC̣ken, Monogr. Dipt. N. Amer., vol. 4, p. 282, 1869. 1 species. Type, Plectromyia modesta Osten Sacken.

Plectropus Haliday, Zool. Journ., vol. 5, p. 353, 1831. 3 species. Type, Мusca pallipes FABricius, the first species, by designation of Westwood, Intr., vol. 2, Synops., 1840, p. 134 (as pallidus Meigen). Not Piectropus Kirby, 1826. Equals Syntormon LoEw, 1857.

$\checkmark \quad$ Plesiastina Winnertz, Ent. Zeit. Stettin, vol. 13, p. 55, 1852. 1 species (as 2). Type, Mycetobia annulata Meigen. Equals Symmerus W ALKer, 1848.

$\checkmark \quad$ Plesiomma Macquart, Dipt. Exot., vol. 1, pt. 2, p. 54; 1838. 2 species. Type, Plesiomma testacea MACQUART, the first species, by designation of $\mathrm{BACK}$, Trans. Amer. Ent. Soc., vol. 35, p. 306, 1909.

Plethochæta Coquillett, Proc. U. S. Nat. Mus., vol. 23, p. 613, 1901. 1 species. Type, Plethochata varicolor Coquilletт.

Plettusa Philippi, Verh. Zool.-Bot. Ges. Wien, vol. 15, p. 597, 1865. 4 species. Type, Plettusa virescens Philippi, the first species, by present designation. Equals Geranomyia Haliday, 1833.

Plinthina Walker, Ins. Saund., Dipt., p. 10, 1850. 1 species. Type, Pangonius macroporum Macquart. Equals Pangonius Latreille, 1802.

Ploas Latreille, Hist. Nat. Crust. et Ins., vol. 14, p. 299, 1805. 1 species. Type, Bombylius virescens FABRICIUs (as hirticornis, new species). Equals Conophorus MeI$\mathrm{GEN}, 1803$. 
Pneumaculex Dyar, Proc. Ent. Soc. Washington, vol. 7, p. 45, 1905.1 species. Type, Culex signifer Coquillett.

Pocota St. Fargeau and Serville, Encycl. Meth., vol. 10, pt. 2, p. 518, 1828. 1 species. Type, Musca apiformis Schrank (as Milesia apicata Meigen). Syn., Dasymyia EqGaer, 1858; Hadromyia WiLliston, 1882.a

Pcecilanthrax Osten SAcken, Biol. Centr.-Amer., Dipt., vol. 1, p. 119, 1886.7 species. Type, Anthrax alcyon $\mathrm{S}_{A Y}$, the first species, by present designation. Equals Villa Lioy, 1864.

Precilognathus J ennicke, Neue Exot. Dipt., p. 42, 1867. 1 species. Type, Pocilognathus thlipsomyzoides J אNNicke. Equals Phthiria Meigen, 1803.

Pogonomyia Rondani, Bull. Soc. Ent. Ital., vol. 2, p. 336, 1870. 1 species. Type, Pogonomyia alpicola Rondan.

Pogonosoma Rondanl, Dipt. Ital. Prodr., vol. 1, p. 160, 1856. 1 species. Type, Asilus maroccanus FABRicius.

Polidea Macquart, Ann. Soc. Ent. France for 1848, p. 92, 1848. 2 species. Type, Tachina ænea Melgen, the first species, by present designation. Equals Lydina Desvoldy, 1830.

Polionoma Rondan1, Dipt. Ital. Prodr., vol. 1, p. 107, 1856. No species. In Atti Soc. Ital. Sci. Nat., vol. 11, 1868, p. 36, he placed it as a synonym of Sapromyza Fallen, 1810. Type, Sapromyza obsoleta Falden. Equals Sapromyza Fallen, 1810.

Polionota WulP, Biol. Centr.-Amer., vol. 2, p. 409, 1899. 2 species. Type, Acrotoxa mucida Giglio-Tos, the first species, by original designation.

Poliophrys Townsend, Smiths. Misc. Coll., vol. 51, p. 90, 1908. 1 species (as 2). Type, Gaediopsis mexicana Brauer and Bergenstamm. Equals Gædiopsis Brauer and Bergenstami, 1891.

Polistomyia Townsend, Smiths. Misc. Coll., vol. 51, p. 132, 1908. 1 species (as 6). Type, Thereva plumipes Fabricius. Equals Trichiopoda Latreille, 1829.

Pollenia Desroldy, Essai Myod., p. 412, 1830. 5 species (as 16). Type, Musca ruats FAmRcius, the fourth species, by orignal designation. Syn., Nitellia Desvoidy, 1830 ; Cephysa Desvoidy, 1863 ; Orizia Desvoldy, 1863.

Polyangæus Doane, Journ. N. Y. Ent. Soc., vol. 8, p. 196, 1900. 1 species. Type, Polyangæus maculatus DoAne.

Polydonta Macquart, Dipt. Exot., Suppl. 4, p. 144, 1850. 1 species. Type, Merodon curvipes IViedenann (as bicolor, new species). Not Polydonta Fischer, 1807. Equals Polydontomyia WiLLIsTon, 1896.

Polydontomyia Williston, Man. N. Amer. Dipt., p. 89, 1896 . Change of name for Polydonta Macquart, 1850, not of Fischer, 1807. Type, Merodon curvipes Wiedemann. Syn., Polydonta Macquart, 1850, preoccupied ; Triodonta Williston, 1885, preoccupied.

Polydromya Bigot, Ann. Soc. Ent. France for 1857, p. 557, 1857. No species. The characters assigned this genus were evidently taken from Meigen's imperfect figure of Tachydromia præcatoria FaLlen (Syst. Beschr. Zweifl. Ins., vol. 3, pl. 23, fig. 13). Type, Tachydromia præcatoria Fallen. Equals Chelifera MaCQUaRT, 1823.

Polygaster Wulp, Biol. Centr.-Amer., Dipt., vol. 2, p. 139, 1890. 1 species. Type, Polygaster egregia WuLP.

Polylepta Winnertz, Verh. Zool.-Bot. Ges. Wien, vol. 13, p. 745, 1863. 2 species. Type, Polylepta undulata WinNerTz, the first species, by designation of JoHANNsen, Gen. Ins., Dipt., Mycet., 1909, p. 43.

Polymeda Meigen, Nouv. Class. Mouch., p.14, 1800.* No species. Erioptera Mrigen, 1803, is a change of name. Type, Erioptera lutea Meigen. Syn., Erioptera Meigen,

$a$ Podoctria MeIGeN, Syst. Beschr. Zweifl. Ins., vol. 2, p. 279, 1820. 5 species. Type, Dasypogon vitripennis MEIGEN, the first species, by present designation. Syn., Isopogon LoEw, 1847. European.

Proc.N.M.vol.37-10-38 
1803; Polygraphia Meigen, 1818; Octavia Bigot, 1854; Chemalida Rondani, 1856; Limncea Rondani, 1856 ; Limnoica Rondani, 1861.

$\checkmark$ Polymedon Osten SAcken, Bull. 3, U. S. Geol. Surv., p. 317, 1877. 1 species. Type, Polymedon flabellifer Osten SAcken.

८ Polymera Wiedemann, Dipt. Exot., p. 40, 1821. 1 species. Type, Chironomus hirticornis FABRICIUS.

$\checkmark$ Polymorphomyia Snow, Kansas Univ. Quart., vol. 2, p. 165, 1894. 1 species. Type, Polymorphomyia basilica Sxow.

$\checkmark$ Polyraphia Meigen, Syst. Beschr. Zweifl. Ins, vol. 1, p. 111, 1818. 1 species. Type, Erioptera trenionota Meigen. Equals Polymeda Meigen, 1800.

$\checkmark$ Pomacera Macquart, Dipt. Exot., Suppl. 2, p. 47, 1847. 1 species. Type, Pomacera bigoti Macquart. Equals Apiocera Westwood, 1835.

$\checkmark$ Popelia Bezzi, Bull. Soc. Ent. Ital., vol. 26, p. 256, 1894. 1 species. Type, Echinomyia popelii Portschinsky. Equals Peleteria Desvoidr, 1830.

$\checkmark$ Porphyrops Meigen, Syst. Beschr. Zweifl. Ins., vol. 4, p. 45, 1824. 29 species. Type, Musca diaphana FABricius, the first species, by designation of Westwood, Intr., vol. 2, Synops., 1840, p. 134. Syn., Argyra MAcquart, 1834; Lasiargyra Mik, 1878. (Porphyrops of authors equals Perithinus HaLiday.)

Porricondyla Rondani, Mem. 2a. Serv. Ditt. Ital., p. 14, 1840.* No species. In his Dipt. Ital. Prodr., vol. 1, 1856, p. 199, 1 species. Type, Cecidomyia albitarsis Meigen.

- Porsenus Darlington, Trans. Amer. Ent. Soc., vol. 34, p. 69, 1908. 1 species. Type, Porsenus johnsoni Darlington.

$\checkmark$ Prionella Desvoidy, Essai Myod., p. 759, 1830. 2 species. Type, Prionella beauvoisii Desvordy, the first species, by present designation.

$\checkmark$ Prionimera Rondani, Dipt. Ital. Prodr., vol. 4, p. 10, 1861. Change of name for Epidesmia Rondani, 185̃6, not of Westwood, 1841. Type, Tephritis cognatus WiedeMann. Equals Acidia Desyoidy, 1830.

$\checkmark$ Prionocera Loew, Ent: Zeit. Stettin, vol. 5, p. 170, 1844. 1 species. Type, Prionocera pubescens Loew. Syn., Stygeropis LoEw, 1863.

$\checkmark$ Prionolabis Osten Sacken, Proc. Acad. Nat. Sci. Phila. for 1859, p. 239, 1860. 1 species. Type, Limnophila rufibasis Osten Sacken. Equals Phylidorea Bigot, 1854.

$\checkmark \quad$ Prionomyia Stephens, Syst. Cat. Brit. Ins., vol. 2, p. 237, 1829. 6 species. Type, Ceratopogon femoratus MEIGEN, the first species, by designation of WEsTwood, Intr., yol. 2, Synops., 1840, p. 126 . Equals Serromyia MeIgen, 1818.

Priophora Desvoidy, Hist. Nat. Dipt., vol. 2, p. 611, 1863. 1 species. Type, Conops irritans Linneus (as Hæmatobia serrata Desvoidy). Equals Hæmatobia St. Fargeau and Serville, 1828.

- Probezzia Kieffer, Gen. Ins., Dipt., Chir., p. 57, 1906. Described as a subgenus of Bezzia, under which genus 44 species were listed without any reference as to which subgenus each belonged. The forty-third species, agreeing with the characters of this subgenus, may be taken as the type. Type, Ceratopogon venusta Meigen. Equals Bezzia KiefFer, 1899.

$\checkmark$ Probolæus Williston, Trans. Ent. Soc. Lond. for 1896, p. 261, 1896. 1 species. Tyne, Probolæus singularis Williston.

$\checkmark$ Proboscimyia Bigot, Bull. Soc. Ent. France for 1883, p. 30, 1883. 1 species. Type, Proboscimyia siphonina Bigot. Syn., Dolichoglossa Stein, 1898.

Prochyliza WaLKer, List Dipt. Brit. Mus., vol. 4, p. 1045, 1849.1 species. Type, Prochyliza xanthostoma WALKer.

Procladius Skuse, Proc. Linn. Soc. N. S. Wales, ser. 2, vol. 4, p. 283, 1889. 2 species. Type, Procladius paludicola Skuse, the first species, by present designation.

Procrita Hendel, Gen. Ins., Dipt., Laux., p. 59, 1908. 1 species. Type, Procrita pectinata HeNDEL. 
Proctacanthus Macquart, Dipt. Exot., vol. 1, pt. 2, p. 120, 1838. 9 species. Type, Proctacanthus philadelphicus MACQUART, the seventh species, by present designation.

Prodiplosis Felt, 23d Rep. State Ent. N. Y., p. 403, 1908. 1 species. Type, Cecidomyia floricola FELT.

$\checkmark$ Promachus Loew, Linn. Ent., vol. 3, p. 390, 1848. 5 species. Type, Asilus maculatus FABRicius, the second species, by present designation. Equals Bactria Meigen, 1820.

Prorates Melander, Ent. News, vol. 17, p. 372, 1906.1 species. Type, Prorates claripennis MeLander.

Prorhynchops Brauer and Bergenstimm, Denkschr. Kais. Akad. Wíss. Wien, vol. 58, p. 364, 1891. 1 species. Type, Prorhynchops bilimeki Brauer and Bergenstamm.

Prosalpia Pokonsy, Wien. Ent. Zeit., vol. 12, p. 54, 1893. 3 species. Type, Anthomyza bilbergi ZetTERstedt (as styriaca, new species), the first species, by designation of Coquillett, Journ. N. Y. Ent. Soc., vol. 9, 1901, p. 140.

- Prosena St. Fargeau and Serville, Encycl. Meth., vol. 10, pt. 2, p. 500, 1828.

1 species. Type, Stomoxys siberita Fabricius. Equals Calirrhoe Meigen, 1800.

$\checkmark$ Prospherysa Wulp, Biol. Centr.-Amer. Dipt., vol. 2, p. 116, 1890. 18 species. Type, Prospherysa æmulans WuLP, the first species, by present designation.

- Protenthes Johannsen, Ent. News, vol. 18, p. 400, 1907. 1 species. Type, Tipula cincta Fabricius (as punctipennis Meigen). Equals Pelopia Meigen, 1800.

Prothecus Rondani, Dipt. Ital. Prodr., vol. 1, p. 139, 1856. 1 species. Type, Pipunculus furcatus EgGer (as Cephalops aucta Fallex). Equals Dorilas Meigen, 1800.

Protocalliphora Hough, Ent. News, vol. 10, p. 66, 1899. 1 species. Type, Musca azurea F Allen. Syn., Avihospita Hendel, 1901.

$\checkmark$ Protoculex Felt, Mosq. Culic. N. Y. State, p. 391d, 1904. 1 species. Type, Culex serratus Theobald. Equals Ochlerotatus Arribalzaga, 1891.

ᄂ Protomacleaya Theobald, Monogr. Culic., vol. 4, p. 253, 1907. 1species. Type, Culex triseriatus Say. Equals Ochlerotatus Arribalzaga, 1891.

Protophormia Townsend, Smiths. Misc. Coll., vol. 51, p.123, 1908. 1 species. Type, Phormia terrænovæ Desvordy. Equals Phormia Desvordy, 1830.

$\checkmark$ Protoplasa Osten SAcken, Proc. Acad. Nat. Sci. Phila. for 1859, p. 251, 1860. 1 species. Type, Protoplasa fitchii Osten Sacken. Syn., Idioplasta Osten Sacken, 1878. Psairoptera W AHLBERG, Kongl. Vet. Akad. Handl. for 1838, p. 18, 1839. 4 species. Type, Homalocephala albitarsis ZETTERstedt (as biumbraia, new species), the second species, by designation of Zetrenstedt, Dipt. Scand., vol. 6, 1847, p. 2264. Equals Homalocephala ZetTerstedt, 1838.

- Psalida Rondani, Dipt. Ital. Prodr., vol. 1, p. 76, 1856. 1 species (in error). In vol. 4, 1861, pp. 91, 92, 2 species (as 3). Type, Ocyptera simplex Fallen, the last species, by designation of Brauer, Verh. Zool.-Bot. Ges. Wien, vol. 43, 1893, p. 495. Equals Leucostoma MeIGEN, 1803

Psectrocladius Kreffer, Mem. Soc. Sci. Bruxelles, vol. 30, p. 356, 1906. 1 species. Type, Orthocladius psilopterus KiefFer. Equals Orthocladius WulP, 1874.

Pselaphephila Becker, Berliner Ent. Zeitschr., vol. 39, p. 122, 1894. 1 species. Type, Pselaphephila loëwi Becker.

Pseudacteon Coquillett, Can. Ent., vol. 39, p. 208, 1907. 1 species. Type, Pseudacteon crawfordii Coquillett.

Pseudapinops Coquillett, Proc. U. S. Nat. Mus., vol. 25, p. 108, 1902. 1 species. Type, Pseudapinops nigra Coquillett.

Pseudatractocera Townsend, Trans. Amer. Ent. Soc., vol. 19, p. 107, 1892. 1.species. Type, Pseudatractocera neomexicana Townsend. 
Pseudatrichia Osten SACKen, Bull. 3, U. S. Geol. Surv., p. 276, 1877. Change of name for Atrichia Loew, 1866, not of Schrank, 1803. Type, Atrichia longurio LoEw. Syn., Atrichia Loew, preoccupied.

Pseudiastata Coquillett, Proc. Ent. Soc. Washington, vol. 9, p. 148, 1908. 1 species. Type, Pseudiastata nebulosa CoquilletT.

$\nu$ Pseudochæta Coquillext, Proc. Acad. Nat. Sci. Phila. for 1895, p. 309, 1895. 1 species. Type, Pseudochæta argentifrons CoquilletT. Syn., Trepophrys Townsend, 1908.

$\checkmark$ Pseudoculex Dyar, Proc. Ent. Soc. Washington, vol. 7, p. 45, 1905. 1 species. Type, Culex aurifer Coquillett. Equals Ochlerotatus Arribalzaga, 1891.

$\checkmark$ Pseudodexia Brauer and Bergenstamm, Denkschr. Kais. Akad. Wiss. Wien, vol. 58, p. 372, 1891.1 species. Type, Dexia eques Wiedemann.

$\checkmark$ Pseudodinia Coquillett, Journ. N. Y. Ent. Soc., vol. 10, p. 187, 1902.1 species. Type, Pseudodinia varipes Coquillett.

$\checkmark$ Pseudogermaria Brauer and Bergenstamm, Denkschr. Kais. Acad. Wiss. Wien, vol. 58, p. 352, 1891. 1 species. Type, Pseudogermaria georgiæ Brauer and BergeNstamm. Equals Distichona WulP, 1890.

$\checkmark$ Pseudogonia Brauer and Bergenstamm, Denkschr. Kais. Akad. Wiss. Wien, vol. 56, p. $100,1889.1$ species. Type, Gonia cinerascens Rondani. Equals Spallanzania Desvoldy, 1830.

Pseudohowardina Theobald, Monogr. Culic., vol. 4, p. 223, 1907. 1 species. Type, Culex trivittata Coquillett. Equals Ochlerotatus Arribalzaga, 1891.

$\checkmark$ Pseudohystricia Brauer and Bergenstamm, Denkschr. Kais. Akad. Wiss. Wien, vol. 56, p. 132, 1889. 1 species. Type, Hystricia ambigua Macquart. Equals Jurinella Brauer and Bergenstamm, 1889.

$\checkmark$ Pseudolfersia Coquilletr, Can. Ent., vol. 31, p. 336, 1899. 1 species. Type, Pseudolfersia maculata Coquillett.

$\checkmark$ Pseudolimnophora Stroвl, Verh. Zool.-Bot. Ges. Wien, vol. 43, p. 272, 1893. 6 species. Type, Musca triangula FALlen, the first species, by designation of CoquilLETT, Journ. N. Y. Ent. Soc., vol. 9, 1901, p. 140. Equals Limnophora Desvoidy, 1830.

$\checkmark$ Pseudomorinia Wulp, Biol. Centr.-Amer. Dipt., vol. 2, p. 259, 1891. 1 species. Type, Pseudomorinia pictipennis WulP.

$\checkmark$ Pseudomyothyria Townsend, Trans. Amer. Ent. Soc., vol. 19, p. 131, 1892. 1 species. Type, Pseudomyothyria indecisa Townsend. Equals Tachinophyto Townsend, 1892.

$\checkmark$ Pseudoperichxta Brauer and Bergenstamm, Denkschr. Kais. Akad. Wiss. Wien, vol. 56, p. 92, 1889. 1 species. Type, Anoxycampta hirta Bigot (as major, new species). Equals Phryxe Desvoidy, 1830.

- Pseudophorocera Brauer and Bergenstamm, Denkschr. Kais. Akad. Wiss. Wien, vol. 56, p. 92, 1889. 1 species. Type, Pseudophorocera setigera Brauer and Bergenstamm. Equals Phryxe Desvoidy, 1830.

- Pseudopyrellia Girschner, Berliner Ent. Zeitschr., vol. 38, p. 306, 1893. 1 species. Type, Musca cornicina Fabricius. Equals Orthellia Desvoidy, 1863.

- Pseudorus Walker, Ins. Saund., Dipt., p. 103, 1851. 1 species. Type, Pseudorus piceus WALKER.

- Pseudotephritis Johnson, Ent. News, vol. 13, p. 144, 1902. Change of name for Stictocephala Loew, 1873, not of Stal, 1869. Type, Ortalis vau SAY. Syn., Stictocephala Loew, 1873, preoccupied.

$\checkmark$ Psila MEIGEN, Illiger's Mag., vol. 2, p. 278, 1803. No species. In his Syst. Beschr. Zweifl. Ins., vol. 5, 1826, pp. 356 to 360,12 species. Type, Musca fimetaria Linnwus, the first species, by designation of Westwoon, Intr., vol. 2, Synops., 1840, p. 146. Syn., Pelethophila Hagenbach, 1822; Psilomyia Latreille, 1829; Ollicia Desvoidy, 1830. 
Psilocephala Zetterstedr, Ins. Lapp., p. 525, 1838. 3 species. Type, Bibio imberbis FALLEN, the second species, by present designation.

Psilocurus Loew, Berliner Ent. Zeitschr, vol. 18, p. 373, 1874. 1 species. Type, Psilocurus nudiusculus Loew. Syn., Orthoneuromyia WILliston, 1893.

$\checkmark$ Psilometopia Liox, Atti Inst. Veneto, ser. 3, vol. 9, p. 990, 1864. 1 species. Type, ?Chortophila crsia Macquart. Equals Pegomya Desvoidy, 1830.

$\checkmark$ Psilomyia Latreille, in Cuvier's Regne Anim., new ed., vol. 5, p. 525, 1829. Change of name for Psila Meigen, 1803, on account of an alleged similar name in the Hemiptera. Type, Musca fimetaria Linneus. Equals Psila Meigen, 1803.

$\checkmark$ Psilopa Fallen, Hydrom. Sveciæ, p. 6, 1823. 4 species. Type, Notiphila nitidula Fallen, the last species, by designation of Rondanl, Dipt. Ital. Prodr., vol. 1, 1856, p. 132. Syn., Hygrella Haliday, 1839; Ephygrobia Schiner, 1862; Diasemocera Bezzi, 1895.

Psilopodinus B1Gor, Bull. Soc. Ent. France for 1888, p. 24, 1888. 1 species. Type, Dolichopus platypterus Fabricius. Equals Sciapus Zeller, 1842. (Psilopodinus of authors equals Megistostylus Bigor.)

- Psilopodius Rondanı, Dipt. Ital. Prodr., vol. 4, p. 11, 1861. Change of name for Psilopus Mrigen, 1824, not of Pol1, 1795. Type, Dolichopus platypterus Fabricius. Equals Sciapus ZelLeR, 1842.

Psiloptera Lioy, Atti Inst. Veneto, ser. 3, vol. 9, p. 906, 1864. 1 species. Type, Musca irritans Fallen. Equals Hydrotæa Desvoldy, 1830.

Psilopus Meigen, Syst. Beschr. Zweifl. Ins., vol. 4, p. 35, 1824.7 species. Type, Dolichopus platypterus FABRICIUs, the second species, by designation of Westwood, Intr., vol. 2, Synops., 1840, p. 134. Not Psilopus Polı, 1795. Equals Sciapus ZELLER, 1842.

Psilosoma Lroy, Atti Inst. Veneto, ser. 3, vol. 9, p. 1118, 1864.1 species. Type, Anthomyza gracilis Fallen. Equals Anthomyza Fallex, 1810.

Psilota Meigen, Syst. Beschr. Zweifl. Ins., vol. 3, p. 256, 1822.1 species. Type, Psilota anthracina MeIGen.

- Psilotanypus KıffFer, Mem. Soc. Sci. Bruxelles, vol. 30, p. 318, 1906. No species. In Gen. Ins., Dipt., Chir., 1906, p. 38, 7 species. Type, Tanypus bellus Loew, the first species, by present designation.

Psorophora Desvoldy, Mem. Soc. Hist. Nat. Paris, vol. 3, p. 412, 1827. 2 species. Ti pe, Culex ciliatus Fabricius, the first species, by designation of Theobald, Monogr. Culicidæ, vol. 1, 1901, p. 263.

Psychoda Latreille, Precis, p. 152, 1796. No species. In his Hist. Nat. Crust. et Ins., vol. 3, 1802, p. 424, 1 species. Type, Tipula phalienoides Linnæus. Syn., Phalænula Meigen, 1800; Trichoptera Meigen, 1803; Tinearia Schellenberg, 1803.

Ptecticus Loew, Verh. Zool.-Bot. Ges. Wien, vol. 5, p. 142, 1855.5 species. Type, Sargus testaceus FABRicius, the last species, by original designation.

Pterallastes Loew, Berliner Ent. Zeitschr., vol. 7, p. 317, 1863. 2 species. Type, Pterallastes thoracicus Loew, the first species, by designation of Osten Sacken, Cat. Dipt. N. Amer., 1878, p. 250.

Pterellipsis Coquilletr, Can. Ent., vol. 31, p. 333, 1899. 1 species. Type, Pterellipsis aranex COQUILLETT.

Pterocalla Rondani, Ins. Ditt. Brasil, p. 83, 1848. 1 species. Type, Dictya ocellata FABRicius.

Pterocera Meigen, Illiger's Mag., vol. 2, p. 275, 1803. 4 species. Type, Musca pellucens Linnæeus, the second species, by present designation. Equals Volucella Geoffroy, 1762.

- Pterocosmus W Alker, List. Dipt. Brit. Mus., vol. 1, p. 78, 1848. 2 species. Type, Pterocosmus hilpa WALker, the second species, by present designation. Equals Caloptera Guerin, 1829. 
$\checkmark$ Pterodontia Gray, in Griffith's Anim. Kingd., vol. 15, Ins., pt. 2, p. 779, 1832. 1 species. Type, Pterodontia flavipes Gray.

Pteroptila Loew, Berliner Ent. Zeitschr., vol. 9, p. 165, 1865. 2 species. Type, Pteroptila decora Loww, the first species, by present designation. Equals Meromacrus Rondani, 1848.

$\checkmark$ Pterospilus Rondani, Dipt. Ital. Prodr., vol. 1, p. 152, 1856. 1 species. Type, Asilus muscarius Fabricius. Equals Acromyia Latreille, 1809.

Ptilodegeeria Brauer and Bergenstanm, Denkschr. Kais. Akad. Wiss. Wien, vol. 58, p. 375, 1891. 1 species. Type, Hypostenc obumbrata WuLP.

$\checkmark$ Ptilodexia Brater and Bergenstamm, Denkschr. Kais. Akad. Wiss. Wien, vol. 56, p. 119, 1889. 1 species. Type, Estheria tibialis Desvordy (as carolinensis, new species). Equals Phorostoma Desvoidy, 1830.

Ptilomyia Coquillett, Proc. U. S. Nat. Mus., vol. 22, p. 261, 1900. 1 species. Type, Ptilomyia enigma Coqulllett.

$\checkmark$ Ptilopareia Brauer and Bergenstamm, Denkschr. Kais. Akad. Wiss. Wien, vol. 56, p. 101, 1889. 1 species. Type, Tachina marginata Meigen. Equals Voria Desvoldy, 1830.

$\checkmark$ Ptilotachina Brauer and Bergenstamm, Denkschr. Kais. Akad. Wiss. Wien, vol. 58, p. 350, 1891. 1 species. Type, Tachina civilis Rondanl. Equals Exorista Meigen, 1803.

$\checkmark$ Ptiolina Zetrenstedt, Dipt. Scand., vol. 1, p. 226, 1842. 2 species. Type, Leptis obscura Fallen, the first species, by designation of Frauenfeld, Verh. Zool.-Bot. Ges. Wien, vol. 5, 1855, p. 497. Syn., Eurytion J.ennicke, 1867.

$\checkmark$ Ptychoproctus Bigot, Rev. May. Zool. for 1859, p. 4, 1859.1 species. Type, Ptychoproctus complexus Bigot. Equals Stylogaster Macquart, 1835.

$\checkmark$ Puchopuligen, Illiger's Mag., vol. 2, p. 262, 1803. 2 species. Type, Tipula contaminala Linneus, the first species, by designation of Latreille, Consider. General., 1810, p. 442. Equals Liriope Meigen, 1800.

I Puliciphora DAHL, Zool. Anzeiger, vol. 20, p. 409, 1897. 1 species. Type, Puliciphora lucifera Dahl. Syn., Stethopathus Wandolleck, 1898; Pachyneurella Brues, 1903.

Pullate Harris, Exp. Engl. Ins., p. 76, 1776. 6 species. Type, Tipula pomonæ Fabricius (as funestus, new species), the second species, by present designation. Equals Bibio Geoffror, 1762.

$\checkmark$ Purpurella Desvoidy, Dipt. Env. Paris, Myop., p. 37, 1853.* 1 species. Type, Purpurella nobilis Desvoidy. Equals Stomoxoides Sch effer, 1766.

$\checkmark$ Pycnoglossa Coquilletт, Proc. U. S. Nat. Mus., vol. 23, p. 613, 1901. 1 species. Type, Pycnoglossa faripennis Coquillett.

- Pycnopogon Loew, Linn. Ent., vol. 2, p. 526, 1847. 4 species. Type, Dasypogon mixtus Loew, the third species, by designation of Rondani, Dipt. Ital. Prodr., vol. 1, 1856 , p. 157.

$\checkmark$ Pycnosoma Brauer and Bergenstamm, Denkschr. Kais. Akad. Wiss. Wien, vol. 61, p. $623,1894.1$ species. Type, Chrysomya regalis Desvoidy (as marginalis W1EDEManN). Equals Chrysomya Desvoidy, 1830.

$\checkmark$ Pygostolus Loew, Berliner Ent. Zeitschr., vol. 10, p. 16, 1866. 3 species. Type, Dasypogon politus SAY (as argentifer, new species). Not Pygostolus HaLIDAY, 1833. Equals Nicocles J ENN ICKE, 1867.

$\checkmark$ Pyrellia Desvoidy, Essai Myod., p. 462, 1830. 3 species (as 9). Type, Musea cadarerina Linn.eus, the second to fourth supposed species, by designation of ZetTERSTEDT, Dipt. Scand., vol. 4, 1845, p. 1320.

Pyrgota Wiedemann, Auss. Zweifl. Ins., vol. 2, p. 580, 1830. 1 species. Type, Pyrgota undata Wiedemann. Syn., Oxycephala Macquart, 1843.

$\checkmark$ Pyritis Hunter, Can. Ent., vol. 29, p. 131, 1897. 1 species. Type, Pyritis montigena Hunter. 
Pyropa SAY, Journ. Acad. Nat. Sci. Phila., vol. 3, p. 98, 1823. 1 species. Type, Pyropa furcata SAY. Equals Scopeuma Melaev, 1800.

Pyrophæna Schıner, Wien. Ent. Monatschr., vol.4, p.213, 1860. 1 species. Type, Syrphus rosarum FABRicius.

$\checkmark$ Pyrrosia Rondani, Dipt. Ital. Prodr., vol. 1, p. 73, 1856. 1 species. Type, Tachina aurea Fallen. Equals Leskia Desvoldy, 1830.

- Quadrula Pandelle, Rev. Ent., vol. 17, p. 51, 1898. 27 species. Type, Anthomyza annosa ZetTeRstedt, the first species, by designation of CoqullLetT, Journ. N. Y. Ent. Soc., vol. 9, 1901, p. 141. Equals Phaonia Desroidy, 1830.

Rabdophaga IVestwood, Gard. Chron. for 1847, p. 588, 1847. 1 species. Type, Cecidomyia (Rabdophaga) viminalis Westwood. Syn., Dichelomyia Rubsanmen, 1892; Bertieria K1efFer, 1896.

Rachicerus Walker, List. Dipt. Brit. Mus., vol. 5, p. 103, 1854. 1 species. Type, Rachicerus fulvicollis W ALKER.

Racodineura Rondanl, Dipt. Ital. Prodr., vol. 4, p. 31, 1861. Change of name for Roeselia Desvoldy, 1830, not of Hüвner, 1816. Type, Tachina antiqua Melgen. Syn., Roeselia Desvoidy, 1830, preoccupied.

Ragheneura Rondan, Dipt. Ital. Prodr., vol. 1, p. 144, 1856. 1 species. Type, Dolichopus griseipennis Stannius. Equals Dolichopus Latreille, 1796.

Rainieria Rondaxi, Nuovi Ann. Sci. Nat. (Bologna), vol. 10,p. 40, 1843. 1 species. Type, Calobata calceata Fallen. Equals Trepidaria Meigen, 1800.

Ramonda Desvoldý, Hist. Nat. Dipt., vol. 1, p. 790, 1863.1 species (as 3). Type, Ramonda fasciata Desvoldy. Equals Wagneria Desvoldy, 1830.

Ravinia Desvoldy, Hist. Nat. Dipt., vol. 2, p. 434, 1863. 2 species (as 4). Type, Sarcophaga hrmatodes Meigen, the second species, by original designation. Equals Sarcophaga Meigen, 1826.

Reaumuria Desvoldy, Essai Myod., p. 79, 1830. 5 species (as 7). Type, Gonia orrata Melgen (as Musca capitata De GeEr), the second species, by designation of Desvoldy, Hist. Nat. Dipt., vol. 1, 1863, p. 733. Equals Salmacia Melgen, 1800.

Redia Lioy, Atti Inst. Veneto, ser. 3, vol. 9, p. 1313, 1864. 4 species. Type, Agromyza gyrans FALLEN, the first species, by present designation. Equals Agromyza FAllen, 1810.

Rhabdopselaphus Biєот, Bull. Soc. Ent. France for 1886, p. 103, 1886.1 species. Type, Rhabdopselaphus mus Bigot.

Rhachoepalpus Townsend, Smiths. Misc. Coll., vol 51, p. 114, 1908. 1 species (as 2). Type, Saundersia testacea Wolp. Equals Epalpus Rondan1, 1850.

Rhadina Kowarz, Wien. Ent. Zeit., vol. 12, p. 144, 1893. 1 species. Type, Chirosia montana Pokorny. Equals Chirosia Rondani, 1856.

Rhadiurgus Loew, Linn. Ent., vol. 4, p. 133, 1849. 1 species. Type, Asilus variabilis ZeTteRstedT.

Rhagio FABricius, Syst. Ent., p. 761, 1775. 4 species. Type, Musca scolopacea Linnæus, the first species, by designation of Latreille, Consider. Général., 1810, p. 443. Syn., Leptis Fabricius, 1805.

Rhagoletis Loew, Monogr. Trypet., p. 44, 1862.* 1 species. Type, Musca cerasi LINNæUS.

Rhamphidia Melgen, Syst. Beschr. Zweifl. Ins., vol. 6, p. 281, 1830. 3 species. Type, Limnobia longirostris Melgen, the first species, by designation of IVESTwood, Intr., vol. 2, Synops., 1840, p. 129 . Equals Megarhina St. Fargead and Serville, 1828.

Rhamphinina BıGoт, Bull. Soc. Ent. France for 1885, p. 11, 1885. 1 species. Type, Rhamphinina dubia Bigot. Equals Phorostoma Desvoidy, 1830.

Rhamphomyia Meigen, Syst. Beschr. Zweifl. Ins., vol. 3, p. 42, 1822.37 species. Type, Empis sulcata Meigen, the ninth species, by designation of Curtis, Brit. Ent., 1834, p. 517. Equals Dionnæa Meigen, 1800. 
Rhaphidolabis Osten SACK EN, Monogr. Dipt. N. Amer., vol. 4, p. 284, 1869. 2 species. Type, Rhaphidolabis tenuipes OsTEN SACKEN, the first species, by original designation.

Rhaphiocera Macquart, Hist. Nat., Dipt., vol. 1, p. 253, 1834. 1 species. Type, Sargus armatus Wiedeman.

$\checkmark$ Rhaphiomidas Osten Sacken, Bull. 3, U. S. Geol. Surv., p. 281, 1877. 1 species. Type, Rhaphiomidas episcopus Osten SaCKen.

$\checkmark$ Rhaphiorhynchus Wiedemann, Dipt. Exot., p. 59, 1821. 1 species. Type, Rhaphiorhynchus planiventris WIEDEMANN.

v [Rhaphium Meigen, Illiger's Mag., vol. 2, p. 272, 1803. No species. In his Syst. Beschr. Zweifl. Ins., vol. 4, 1824, pp. 28 to 31, 9 species. Type, Rhaphium macrocerum Meigen, the third species, by designation of CurTis, Brit. Ent., 1835, p. 568. Syn., Xiphandrium Loew, 1857. Not American.]

$\checkmark$ Rhedia Desvoiny, Essai Myod., p. 74, 1830. 5 species (as 9). Type, Gonia atra Meigen (as vicina, new species), the third species, by present designation. Equals Salmacia Meigen, 1800.

$\checkmark$ Rhicnœssa Loew, Wien. Ent. Monatschr., vol. 6, p. 174, 1862. 1 species. Type, R̆hicnossa cinerea LoEw.

$\checkmark$ Rhingia Scopoli, Ent. Carn., p. 358, 1763. 1 species. Type, Rhingia austriaca Meigen (as Conops rostrata Linneus).

$\checkmark \quad$ Rhinomya Desvoiny, Essai Myod., p. 123, 1830. 1 species. Type, Rhinomya gagatea Desvoidy. Equals Rhinophora Desvoidy, 1830 .

Rhinophora Desvoiny, Essai Myod., p. 258, 1830. 2 species (as 6). Type, Tachina gagatina MEIGEN (equaling the supposed species 2 to 6 ), by designation of Desvoldy, Hist. Nat. Dipt., vol. 2, 1863, p. 5. Syn., Kirbya Desvoiny, 1830; Rhinomya Desvoidy, 1830; Cassidæmyia Macquart, 1835; Chista Melgen, 1838; Microcheilosia Macquart, 1855 .

Rhinoplecia Bellard, Mem. Reale Accad. Sci. Torino, ser. 2, vol. 19, p. 16, 1859.

1 species. Type, Plecia rostellata Loew (as rostrata, new species). Equals Plecia WIEDEMANN, 1828.

$\checkmark$ Rhinotora Schiner, Reise Novara, Zool., vol. 2, Dipt., p. 233, 1868. 2 species. Type, Rhinotora phricellata Schiner, the first species, by original designation.

$\checkmark \quad$ Rhipidia Meigen, Syst. Beschr. Zweifl. Ins., vol. 1, p. 153, 1818. 1 species. Type, Rhipidia maculata Meigen.

$\checkmark$ Rhodogyne Mleigen, Nouv. Class. Mouch., p. 39, 1800.* No species. Gymnosoma Meigen, 1803, is a change of name. Type, Musca rotundata Linnæus. Syn., Gymnosoma Meigen, 1803.

Rhombothyria WulP, Biol. Centr.-Amer. Dipt., vol. 2, p. 259, 1891.1 species. Type, Rhombothyria flavicosta WULP.

- Rhopalomyia Rubsaanen, Berliner Ent. Zeitschr., vol. 37, p. 370, 1892.8 species. Type, Origotrophus tanaceticola KARsCH, the first species, by designation of KIEFFER, Wien. Ent. Zeit., vol. 15, 1896, p. 89.

$\checkmark \quad$ Rhopalomyia Williston, Psyche, vol. 7, p. 184, 1895. 1 species. Type, Ropalomera pleuropunctata Wiedemann. Not Rhopalomyia RÜBsaAmen, $1892 . \quad$ Equals Willistoniella Mıк, 1895.

Rhopalosyrphus Giglio-Tos, Boll. Mus. Zool. Univ. Torino, vol. 6, No. 108, p. 3 , September 20, 1891 . No species. In vol. 7, No. 118, March 24, 1892, p. 1, 1 species. Type, Holmbergia guntheri Arribalzaga. Syn., Holmbergia Arribalzaga, October, 1891.

[Rhynchiodexia Bigot, Bull. Soc. Ent. France for 1885, p. 11, 1885. 1 species. Type, Rhynchiodexia tenuipes Bigot. Not American.] (Rhynchiodexia of authors equals Phorostoma Desvoidy.)

$\checkmark$ Rhynchocephalus Fischer, Mem. Soc. Imper. Moscow, vol. 1, p. 217, 1806.* 3 species (as 4). Type, Rhynchocephalus tauscheri Fischer, the last species, by desig- 
nation of SchlNer, Reise Novara, Zool., vol. 2, 1868, p. 107. Syn., Andrenomya RoNDANI, 1850.

$\checkmark$ Rhynchogonia Brauer and Bergenstama, Denkschr. Kais. Akad. Wiss. Wien, vol. 60 , p. $125,1893.1$ species. Type, Rhynchogonia algerica Brauer and Bergenstamm. Equals Salmacia Melgen, 1800.

Rhynchomilichia Hendel, Wien. Ent. Zeit., vol. 22, p. 250, 1903.1 species. Type, Lobioptera argyrophenga ScHiner.

- Rhynchosciara RubsaAmen, Berliner Ent. Zeitschr., vol. 39, p. 29, 1894. 2 species. Type, Rhynchosciara villosa RuBsaAmen, the first species, by present designation.

Rhynchosia Macquart, Ann. Soc. Ent. France for 1848, p. 87, 1848. Change of name for Olivieria Melgen, 1838, not of Desvoldy, 1830. Type, Tachina longirostris Meigen. Equals Aphria Desvoidy, 1830.

Rhynchotrichops Schnabl, Hor. Soc. Ent. Ross., vol. 23, p. 344, 1889. 4 species. Type, Anthomyza aculeipes ZETTERSTEDT, the first species, by designation of CoqUiLLETT, Journ. N. Y. Ent. Soc., vol. 9, 1901, p. 141.

Rhyphus Jatreille, Hist. Nat. Crust. et Ins., vol. 14, p. 291, 1805.1 species. Type, Tipula fenestralis Scopoli (as fenestrarum Scopolı). Equals Sylvicolæ HarRIS, 1776.

Rhysops Williston, Journ. N. Y. Ent. Soc., vol. 15, p. 2, 1907. 3 species. Type, Melanostoma rugonasus Willıston, the second species, by present designation.

Richardia Desvoldy, Essai Myod., p. 728, 1830. 1 species. Type, Dacus podagrica Fabricius (as saltatoria, new species). Syn., Merodina Macquart, 1835. Rileya Brauer and Bergenstamm, Denkschr. Kais. Akad. Wiss. Wien, vol. 60, p. 121, 1893. 1 species. Type, Blepharipeza adusta Loew (as americana, new species). Equals Blepharipeza Macquart, 1843.

Rileymyia Townsend, Ent. News, vol. 4, p. 277, 1893. Change of name for Rileya Brauer and Bergenstamm, 1893, preoccupied.. Type, Blepharipeza adusta Loew. Equals Blepharipeza MaCqUart, 1843.

Rivellia Desvoldy, Essai Myod., p. 729, 1830. 3 species. Type, Musca syngenesix FABRICIUS (as herbarum, new species), the first species, by designation of RoNDANI, Dipt. Ital. Prodr., vol. 1;1856, p. 111.

Rœderiodes CoquilletT, Bull. 47, N. Y. State Mus., p. 585, 1901. 1 species. Type, Roederiodes juncta CoquILlETT.

Roeselia Desvoiny, Essai Myod., p. 145, 1830. 1 species (as 4). Type,Tachina antiqua Meigen. Not Roselia Hübner, 1816. Equals Racodineura Rondanl, 1861.

Rohrella Drsvoldy, Essai Myod., p. 489, 1830. 9 species. Type, Musca pallida Fabricius (as fragilis, new species), the seventh species, by designation of CoquilLETT, Journ. N. Y. Ent. Soc., vol. 9, 1901, p. 141. Equals Phaonia Desvoldy, 1830.

Romaleosyrphus BıGot, Bull. Soc. Ent. France for 1882, p. 129, 1882.1 species. Type, Romaleosyrphus villosus Bigot. Equals Crioprora Osten Sacken, 1878.

Rondania J Ennicke, Abh. Sencken. Ges., vol. 6, p. 324, 1867. 1 species. Type, Rondania obscura J enNicke. Not Rondania Desvo1Dy, 1850. Equals Neorondania OsTen SACKEN, 1878.

Rondaniella Johannsen, Gen. Ins., Dipt., Mycet., p. 66, 1909. 9 species. Type, Leia variegata WINNERTZ, the last species, by original designation.

Rondanimyia Townsend, Smiths. Misc. Coll., vol. 51, p. 67, 1908. Change of name for Gymnopsis Rondanl, 1859, not of Rafinesque, 1815. Type, Tachina chalconota Meigen. Equals Macquartia Desvoldy, 1830.

Ropalocera Melgen, Syst. Beschr. Zweifl. Ins., vol. 2, p. 302, 1820. 1 species. Type, Laphria nigripennis Meigen. Equals Laphria Meigen, 1800.

Ropalomera Wiedemann, Anal. Ent., p. 17, 1824.* 4 species. Type, Dictya clavipes FABriclus, the first species, by designation of MACQUART, Dipt. Exot., vol. 2, pt. 3, 1843, p. 359 . 
Rymosia Winnertz, Verh. Zool.-Bot. Ges. Wien, vol. 13, p. 810,1863. 13 species. Type, Mycetophila fasciata Meigen, the first species, by designation of JoHannsen, Gen. Ins., Dipt., Mycet., 1909, p. 102.

$\checkmark$ Rypholophus Kolenati, Wien. Ent. Monatschr., vol. 4, p. 393, 1860. 1 species. Type, Rypholophus phryganoptemis Kolenati. Equals Ormosia Rondant, 1856.

$\checkmark$ Sabethes Desvoidy, Mem. Soc. Hist. Nat. Paris, vol. 3, p. 411, 1827. 1 species. Type, Culex cyaneus Fabricius (as locuples, new species).

$\checkmark$ Sabethoides Theobald, Monogr. Culic., vol. 3, p. 328, 1903.1 species. Tvpe, Sabethoides confusus TнеовацD.

J Sackenomyia Felt, 23d Rep. State Ent. N. Y., p.361, 1908. 1 species. Tỳpe, Oligotrophus acerifolius FELT.

Sagaris Desvoiny, Hist. Nat. Dipt., vol. 1, p. 486, 1863. 1 species (as 3). Type, Musca libatrix Panzer. Equals Zenilla Desvoidy, 1830.

$\checkmark$ Salia Desvoidy, Essai Myod., p. 108, 1830. 3 species (as 8). Type, Salia echinura Desvordy, the third species, by designation of Desvordy, Hist. Nat. Dipt., vol. 1, 1863 , p. 553 . Equals Phorocera Desvoldy, 1830.

$\checkmark$ Salmacia Meigen, Nouv. Class. Mouch., p. 38, 1800.* No species. Gonia MeIGEN, 1803, is a change of name. Type, Musca capitata De Geer. Syn., Gonia Meigen, 1803; Rhedia Desvoidy, 1830; Reaumuria Desvoldy, 1830; Isomera Desvoidy, 1851; Onychogonia Brauer and Bergenstamm, 1889; Rhynchogonia Brauer and Bergenstami, 1893.

v Salpingogaster Schiner, Reise Novara, Zool., vol. 2, Dipt., p. 344, 1868. 3 species. Type, Salpingogaster pygophora SCHINER, the first species, by original'designation.

$\checkmark$ Sapromyza Fallex, Spec. Ent. Meth. Exh., p. 18, 1810. 1 species. Type, Sapromyza obsoleta FAlden (as Tephritis flava Fabricius). Syn., Sylvia Desvoidy, 1830; Lycia Desvoidy, 1830; Terenia Desvoidy, 1830; Minettia Desvoidy, 1830; Cnemacantha Macquart, 1835; Polionoma Rondani, 1856; Sapromyzosoma Liox, 1864; Stylocoma Lioy, 1864.

- Sapromyzosoma Lioy, Atti Inst. Veneto, ser. 3, vol. 9, p. 1009, 1864. 1 species. Type, Musca quadripunctata Linneus (as Sapromyza tibialis Macquart). Equals Sapromyza FALLEN, 1810.

$\checkmark$ Sarcionus Aldrich, Biol. Centr.-Amer. Dipt., vol. 1, p. 341, 1901.2 species. Type, Pelastoneurus lineatus AldRICH, the first species, by original designation.

$\checkmark$ Sarcoclista Townsend, Trans. Amer. Ent. Soc., vol. 19, p. 122, 1892.1 species. Type, Sarcoclista dakotensis Townsend.

$\checkmark$ Sarcodexia Townsend, Journ. Inst. Jamaica, vol. 1, p. 105, 1892. 1 species. Type, Sarcodexia sternodontis TownsEnd.

$\checkmark$ Sarcomacronychia Townsend, Trans. Amer. Ent. Soc., vol. 19, p. 100, 1892.1 species. Type, Pachyophthalmus floridensis Townsend (as unica, new species). Equals Pachyophthalmus Brauer and Bergenstami, 1889.

$\checkmark$ Sarcophaga Meigen, Syst. Beschr. Zweifl. Ins., vol. 5, p. 14, 1826. 30 species. Type, Musca carnaria Linneus, the sixth species, by designation of Westwood, Intr., vol. 2, Synops., 1840, p. 140 . Syn., Myophora Desvoldy, 1830; Phorella Desvoidy, 1830; Agria Desvoidy, 1830; Pierretia Desvoidx, 1863; Sertaisia Desvordy, 1863; Bellieria Desvoidy, 1863; Ravinia Desvoidy, 1863; Scaligeria Desvordy, 1863; Bercea Desvoidy, 1863; Calyptia Desvoldr, 1863; Listeria Desvoldy, 1863; Sfarogaster Lioy, 1864 .

Sarcophagula WulP, Tijdschr. Ent., vol. 30, p. 173, 1887. 6 species. Type, Musca occidua $\mathrm{F}_{\mathrm{ABRI}} \mathrm{IUS}$, the first species, by present designation.

Sarcophila Rondani, Dipt. Ital. Prodr., vol. 1, p. 86, 1856. 1 species. Type, Musca latifrons Fallen. Syn., Wohlfahtia Brauer and Bergenstami, 1889.

$\checkmark$ Sarcophiloides Brauer and Bergenstamm, Denkschr. Kais. Akad. Wiss. Wien. vol. 56, p. 164, 1889. 1 species. Type, Tachina pusilla Wiedenann. 
Sarcotachinella Townsend, Trans. Amer. Ent. Soc., vol. 19, p. 110, 1892.1 species. Type, Sarcotachinella intermedia Townsend. Equals Brachicoma Rondani, 1856.

Sardiocera Brauer and Bergenstami, Denkschr. Kais. Akad. Wiss. Wien, vol. 56, p. 119, 1889. 1 species. Type, Theresia tandrec Desvordy (as valida, new species). Equals Theresia Desvordy, 1830.

Sargus Fabricius, Ent. Syst., Suppl., p. 566, 1798. 5 species. Type, Musca cuprarius LinN us, the first species, by designation of Latreille, Consider. Général., 1810, p. 442. Not Sargus WALB, 1792. Equals Geosargus BeZZI, 1907.

Saropogon Loew, Linn. Ent., vol. 2, p. 439, 1847. 4 species. Type, Dasypogon luctuosus Meigen, the last species, by present designation.

$\checkmark$ Sarothromyia Brauer and Bergenstami, Denkschr. Kais. Akad. Wiss. Wien, vol. 58, p. 365, 1891.1 species. Type, Sarcophila femoralis Schiner.

Satyra Meigen, Illiger's Mag., vol. 2, p. 272, 1803. 1 species. Type, Musca ungulata Linneus. Equals Dolichopus Latreille, 1796.

Saucropus Loww, Neue Beitr., vol. 5, p. 41, 1857. 4 species. Type, Dolichopus suturalis FALLEN, the third species, by present designation. Equals Neurigona Rondani, 1856.

Saundersia Schiner, Reise Novara, Zool., vol. 2, Dipt., p. 333, 1868. 1 species. Type, Micropalpus ornatus Macquart. Equals Epalpus Rondani, 1850.

Savia Rondani, Dipt. Ital. Prodr., vol. 4, p. 140, 1861. 1 species. Type, Tachina melanocephala Meigen. Equals Phyto Desvoidy, 1830.

Sayomyia Coquillett, Can. Ent., vol. 35, p. 190, 1903. 1 species. Type, Corethra punctipennis SAY. Equals Chaoborus Lichtenstein, 1800.

Scæva Fabricius, Syst. Antliatorum, p. 248, 1805. 25 species. Type, Musca pyrastri Linnæus, the third species, by designation of CurTis, Brit. Ent., 1834, p. 509. Syn., Lasiophthicus Roxdani, 1844; Catabomba Osten Sacken, 1877.

Scaligeria Desvordy, Hist. Nat. Dipt., vol. 2, p. 476, 1863. 2 species (as 9). Type, Sarcophaga melanura Meigen, the second species, by original designation. Equals Sarcophaga MEIGEN, 1826.

Scaptia Walker, Ins. Saund., Dipt., p. 8, 1850. 5 species. Type, Pangonius auratus Macquart, the first species, by present designation. Equals Pangonius LATREILLE, 1802.

Scaptomyza Hardx, Proc. Berwick Nat. Club, p. 349, 1849.* 2 species. Type, Drosophila graminum FALLEN, the first species, by present designation.

Scarphia Walker, Ins. Saund., Dipt., p. 10, 1850. 1 species. Type, Pangonius directa Walker. Equals Pangonius Latreille, 1802.

Scatella Desvordy, Essai Myod., p. 801, 1830. 2 species. Type, Ephydra stagnalis Fallen (as buccata, new species), the first species, by designation of BECKER, Berliner Ent. Zeitschr., vol. 41, 1896, p. 224 . Syn., Trixostomus Rondani, 1856.

$\checkmark$ Scathophaga.Meigen, Illiger's Mag., vol. 2, p. 277, 1803. 1 species. Type, Musca merdaria Fabricius. Equals Scopeuma Meigen, 1800.

' Scathopse Geofrroy, Hist. Abrégée Ins., vol. 2, p. 544, 1762. 2 species (without names). Type, Tipula notata Linndeus, the first species, by designation of LATREILLE, Consider. General., 1810, p. 442 (as albipennis Fabricius). Syn., Ceria Scopoli, 1763.

Scatina Desvordy, Essai Myod., p. 629, 1830. 1 species. Type, Scatina claripennis Desvoidy. Equals Scopeuma Meigen, 1800.

Scatomyza Fallen, Spec. Ent. Meth. Exh., p. 15, 1810. 2 species. Type, Musca scybalaria Linneus, the first species, by present designation. Equals Scopeuma MeIgeN, 1800.

Scellus Loew, Neue Beitr., vol. 5, p. 22, 1857. 2 species. Type, Hydrophorus spinimanus ZetTERstedt, the second species, by present designation. Syn., Anoplomerus RoNDANI, 1856, preoccupied. 
$\checkmark$ Scenopinus Latreille, Hist. Nat. Crust. et Ins., vol. 3, p. 463, 1802. 1 species. Type, Musca fenestralis Linneus. Equals Omphrale Meigen, 1800.

$\checkmark \quad$ Schaumia Desvordy, Hist. Nat. Dipt., vol. 2, p. 43, 1863. 1 species. Type, Tachina bimaculata Hartig. Equals Sturmia Desvoidy, 1830.

$\checkmark$ Schizomyia KiefFer, Ent. Nachr, vol. 15, p. 183, 1889. 1 species. Type, Schizomyia galiorum KIEFFER.

$\checkmark \quad$ Schizotachina Walker, Ins. Saund., Dipt., p. 264, 1852. 1 species (as 2). Type, Tachina convecta WALKER.

$\checkmark$ Schœnomyza Haliday, Ent. Mag., vol. 1, p. 166, 1833. 1 species (as 2). Type, Ochitiphila litorella Falden. Syn., Litorella Rondani, 1856.

$\checkmark$ Sciapus Zellek, Isis von Oken for 1842, p. 831, 1842. Change of name for Psilopus ME1GEN, 1824, under the erroneous impression that it is preoccupied by Psilopa FALLEN, 1823. Type, Dolichopus platypterus Fabricius. Syn., Leptopus Fallen, 1823, preoccupied; Psilopus Meigen, 1824, preoccupied; Psilopodius Rondani, 1861; Psilopodinus Bigot, 1888; Gnamptopsilopus ALDRICH, 1893.

$\checkmark \quad$ Sciara Meigen, Illiger's Mag., vol. 2, p. 263, 1803. 1 species. Type, Tipula thomæ Linneus. Equals Lycoria Meigen, 1800.

$\checkmark \quad$ Sciasma Coquillett, Rev. Tach., p. 69, 1897. 1 species. Type, Sciasma nebulosa Coquillett.

$\checkmark$ Sciodromia Haliday, in Westwood's Intr., vol. 2, Synops., p. 132, 1840. 1 species. Type, Heleodromia immaculata Haliday. Equals Heleodromia Haliday, 1833.

$\checkmark$ Sciomyza Fallen, Sciomyzides Sveciæ, p. 11, 1820. 12 species. Type, Sciomyza simplex Fallex, the first species, by designation of Wesrwood, Intr., vol. 2, Synops., 1840, p. 145. Syn., Bischofia Hendel, 1902. (Sciomyza of authors equals Chetocera Desvoidy.)

$\checkmark$ Scione W Alker, Ins. Saund., Dipt., p. 10, 1850. 1 species. Type, Pangonius incompletus Macquart. Syn., Diclisa Schiner, 1867.

$\checkmark$ Sciophila Meig ex, Syst. Beschr. Zweifl. Ins., vol. 1, p. 245, 1818. 13 species. Type, Sciophila hirta Meigen, the twelfth species, by designation of Curtis, Brit. Ent., 1837, p. 641. Syn., Lasiosoma Winnertz, 1863. (Sciophila of authors equals Mycomya RONDANI.)

$\checkmark$ Scleropogon Lokw, Berliner Ent. Zeitschr., vol. 10, p. 26, 1866. 1 species. Type, Scleropogon picticornis Loew. Equals Stenopogon Loew, 1847.

$\checkmark$ Scoliocentra Loew, Zeitschr. Ent. Breslau, vol. 13, p. 43, 1862. 1 species. Type, Heleomyza villosa Meigen.

$\checkmark$ Scoliopelta Williston, Ent. Amer., vol. 1, p. 154, 1885. 1 species. Type, Scoliopelta luteipes WiLliston.

$\checkmark$ Scopeuma Meigen, Nouv. Class. Mouch., p. 36, 1800* No species. Scathophaga Meig EN, 1803, is a change of name. Type, Musca merdaria Fabricius. Syn., Scathophaga Meigen, 1803; Scatomyza Fallen, 1810; Pyropa SAY, 1823; Amina Desvoldy, 1830; Scatina Desvoldy, 1830.

J Scopolia Desvordy, Essai Myod., p. 268, 1830. 3 species (as 5). Type, Musca carbonaria PAnzer, the second species, by designation of ZetTerstedt, Dipt. Scand., vol. 3, 1844, p. 1239 . Equals Wagneria Desvo1Dy, 1830.

Scotia Desvoldy, Hist. Nat. Dipt., vol. 1, p. 255, 1863. 1 species (as 2). Type, Tachina grandis Zetterstedt. Equals Thrycolyga Rondani, 1856.

$\checkmark$ Scotiptera Macquart, Hist. Nat., Dipt., vol. 2, p. 215, 1835. 2 species. Type, Sophia punctata Desvoidy, the second species, by designation of Macquart, Dipt. Exot., vol. 2, pt. 3, 1843, p. 240 (as Dexia melaleuca Wiedemans).

$\checkmark$ Scutops Coquilletr, Proc. Ent. Soc. Washington, vol. 6, p. 97, 1904. 1 species. Type, Scutops fascipennis CoquILleTt.

$\checkmark$ Scyphella Desvoidy, Essai Myod., p. 650, 1830. 2 species. Type, Musca flara Linneus (as flavicornis, new species), the first species, by designation of BezzI, Atti Soc. Ital. Sci., vol. 43, 1904, p. 178. Equals Chyromya Desvo1dy, 1830. 
Seilopogon Costa, Ann. Sci. Napoli, vol. 1, p. 74, 1854.* 1 species. Type, Asilus diadema Fabricius (as sicanus, new species). Equals Dasypogon Meigen, 1803.

Seioptera Kirby, An Intr. Ent., vol. 2, p. 305, 1817. 1 species. Type, Musca vibrans Linnæus. Equals Ortalis Falden, 1810.

Semiomyia Bremi, Ann. Soc. Ent. France for 1848, pp. 102, 103, 1848. 1 species (as 3). Type, Crameria oestroidea Desvoidy. Equals Trixa Meigen, 1824.

Senogaster Macquart, Hist. Nat., Dipt., vol. 1, p. 519, 1834.1 species. Type, Senogaster corulescens Macquart. Syn., Acrochordonodes Bigot, 1878.

Senopterina Macquart, Hist. Nat., Dipt., vol. 2, p. 453, 1835. 1 species. Type, Dacus brevipes FABRICIUS.

Senotainia Macquart, Dipt. Exot., Suppl. 1, p. 167, 1846. 1 species. Type, Senotainia mubriventris Macquart. Syn., Arrenopus Brauer and Bergenstamm, 1891.

V Sepedon Latreille, Hist. Nat. Crust. et Ins., vol. 14, p. 385, 1805. 1 species. Type, Syrphus sphegeus FABRicius.

Sepsis Fallen, Spec. Ent. Meth. Exh., p. 17, 1810. 2 species. Type, Musca cynipsea Linneus, the second species, by designation of Curtis, Brit. Ent., 1829, p. 245. Syn., Acrometopia Lioy, 1864; Beggiatia Lioy, 1864.

Sepsisoma Johnson, Ent. News, vol. 11, p. 327, 1900. 1 species. Type, Sepsisoma flavescens JoHnson.

Sericomyia Mergen, Illiger's Mag., vol. 2, p. 274, 1803. 2 species. Type, Musca lappona Linnaus, the second species, by designation of Latreille, Consider. Général., 1810, p. 443. Equals Cinxia Meigen, 1800.

Serromyia Meigen, Syst. Beschr. Zweifl. Ins., vol. 1, p. 83, 1818. 1 species. Type, Ceratopogon femoratus Meigen. Syn., Prionomyia Stephens, 1829; Ceratolophus KiEFFER, 1899; Johannseniella Williston, 1907.

Servaisia Desvordy, Hist. Nat. Dipt., vol. 2, p. 429, 1863. 1 species (as 5). Type, Sarcophaga erythrura Meigen. Equals Sarcophaga Meigen, 1826.

Sfærogaster Lioy, Atti Inst. Veneto, ser. 3, vol. 9, p. 889, 1864. 1 species. Type, Sarcophaga laticornis Meigen. Equals Sarcophaga Meigen, 1826.

Siagona Meigen, Syst. Beschr. Zweifl. Ins., vol. 6, pl. 65, figs. 5, 6, and 7, 1830. 1 species. Type, Glochina sericata Meigen. Equals Furcomyia Meigen, 1818.

SicuaScopoli, Ent. Carn., p. 369, 1763. 2 species. Type, Conops ferruginea LinN

Sicus Latreille, Precis, p. 158, 1796 . No species. In his Hist. Nat. Crust. et Ins., vol. 14, 1805, p. 312, 1 species. Type, Musca arrogans Linneus (as cimicoides Fabricius). Not Sicus Scopoli, 1763. Equals Coryneta Meigen, 1800.

Sigaloëssa Loew, Berliner Ent. Zeitschr., vol. 9, p. 186, 1865. 1 species. Type, Sigaloëssa bicolor Loew.

Sigmatomera Osten SACken, Monogr. Dipt. N. Amer., vol. 4, p. 137, 1869. No species. In 1873, 1 species. Type, Sigmatomera flavipennis Osten SaCken.

Siligo Aldrich, Trans. Amer. Ent. Soc., vol. 34, p. 98, 1908. '2 species. Type, Siligo oregona ALDRICH, the first species, by original designation. Equals $Z$ agonia Coquillett, 1904.

Silvius Meigen, Syst. Beschr. Zweifl. Ins., vol. 2, p. 27, 1820. 1 species. Type, Tabanus vituli FABRICIUS.

Simondella Laveran, Compt. Rend. Soc. Biol. for 1902, p. 1160, 1902.1 species. Type, Simondella curvirostris Laveran. Equals Limatus Theobald, 1901.

Simulium Latreille, Hist. Nat. Crust. et Ins., vol. 3, p, 426, 1802. 1 species. Type, Rhagio colombaschensis Fabricius. Equals Molusina Meigen, 1800.

Sinophthalmus Coquillett, Proc. Ent. Soc. Washington, vol. 6, p. 190, 1904. 1 gpecies. Type, Sinophthalmus pictus Coquillett.

Siphoclytia Townsend, Trans. Amer. Ent. Soc., vol. 19, p. 116, 1892. 1 species. Type, Siphoclytia robertsonii Townsend. Equals Epigrimyia Townsend, 1891. 
$\checkmark$ Siphomyia Williston, Trans. Ent. Soc. Lond. for 1896, p. 418, 1896. 2 species. Type, Hippelates proboscideus Williston, the first species, by present designation.

$\checkmark$ Siphona Meigen, Illiger's Mag., vol. 2, p. 281, 1803. 1 species. Type, Muscageniculata DEGEER (as Stomoxys irritans FABRICIUS, an error for minuta FABRICIUs). Equals Crocuta Meigen, 1800.

$\checkmark$ Siphonella Macquart, Hist. Nat., Dipt., vol. 2, p. 584, 1835.4 species. Type, Oscinis oscinina FaLlen, the first species, by original designation. Equals Madiza FALLEN, 1810.

$\checkmark$ Siphoniomyia Вigot, Bull. Soc. Ent. France for 1885, p. 12, 1885. 1 species. Type, Trichophora analis Schiner (as melas, new species). Equals Trichophora MACQUART, 1847.

$\checkmark \quad$ Siphophyto Townsend, Trans. Amer. Ent. Soc., vol. 19, p. 127, 1892. 1 species. Type, Siphophyto floridensis Townsend. Equals Epigrimyia Townsend, 1891.

$\checkmark$ Siphoplagia Townsend, Trans. Amer. Ent. Soc., vol. 18, p. 349, 1891. 1 species. Type, Siphoplagia anomala Townsend.

$\checkmark$ Siphosturmia Coqurllett, Rev. Tach., p. 37, 1897. 1 species. Type, Argyrophylax rostrata CoQUILLETT.

$\checkmark$ Siphunculina Rondani, Dipt. Ital. Prodr., vol. 1, p. 128, 1856. 1 species. Type, Siphunculina brevinervis Rondani. Equals Madiza Fallen, 1810.

$\checkmark \quad$ Sisyropa Brauer and Bergenstamm, Denkschr. Kais. Akad. Wiss. Wien, vol. 56, p. 163, 1889. 1 species. Type, Tachina thermophila Wiedemann. Equals Carcelia DEsTOIDY, 1830.

$\checkmark \quad$ Sitarea Desvoldr, Essai Myod., p. 763, 1830. 2 species. Type, Sitarea scorzoneræ Desvoruy, the first species, by present designation. Equals Terellia Desvordy, 1030.

Snowiellus Hrne, Ohio Nat., vol. 5, p. 230, 1904. 1 species. Type, Snowiellus atratus Hine.

$\nu$ Solieria Desvordy, Ann. Soc. Ent. France for 1848, p. 461, 1848. 5 species (as 21). Type, Tachina inanis Fallen (equaling 9 of the supposed species), by present designation. Equals Leskia Desvordy, 1830.

$\checkmark \quad$ Solva W ALker, Journ. Proc. Linn. Soc., Zool., vol.4, p. 98, 1860. 1 species. Type, Solva inamœena Walker. Syn., Subula Meigen, 1820, preoccupied; Xylomya RonDANI, 1861; Macroceromys Bigot, 1877.

$\checkmark$ Somoleja Rondani, Atti Soc. Ital. Sci. Nat., vol. 8, p. 207, 1865. 1 species. Type, Tachina anea Meigen (as Harrisia rebaptizata Rondani). Equals Lydina Desvoidy, 1830.

$\checkmark$ Somomya Rondani, Dipt. Ital. Prodr., vol. 4, p. 9, 1861. Change of name for Mya Rondani, 1850, not of Linneus, 1758. Type, Musca segmentaria Fabricius. Equals Lucilia Desvoidy, 1830.

$\checkmark$ Somula Macquart, Dipt. Exot., Suppl. 2, p. 57, 1847. 1 species. Type, Somula decora Macquart. Equals Penthesilia Meigen, 1800.

$\checkmark$ Spallanzania Desvoldr, Essai Myod., p. 78, 1830. 2 species. Type, Tachina hebes Fallen (as gallica, new species), the second species, by present designation. Syn., Pissemya Desvoidy, 1851; Chephalia Rondani, 1856; Pseudogonia Brauer and BergenSTAMм, 1889; Acroglossa. Williston, 1889.

$\checkmark \quad$ [Spania Meigen, Syst. Beschr. Zweifl. Ins., vol. 6, p. 335, 1830. 1 species. Type, Spania nigra Meigen. Not American.]

$\checkmark$ Spanipalpis Townsend, Smiths. Misc. Coll., vol. 51, p. 110, 1908. 1 species. Type, Trichophora miscella Coqulllett. ·

$\checkmark$ Sparnopolius Loew, Neue Beitr., vol. 3, p. 43, 1855. 4 species. Type, Bombylius brevirostris Macquart (as fuluus WiedemanN, preoccupied), the first species, by present designation.

$\checkmark$ Spathichira Bigot, Bull. Soc. Ent. France for 1888, p. 24, 1888. 1 species. Type, Dolichopus funditor Loew. Equals Dolichopus Latreille, 1796. 
Spathulina Rondani, Dipt. Ital. Prodr., vol. 1, p. 113, 1856. 1 species. Type, Spathulina sicula Rondani. Syn., Campiglossa Rondani, 1870.

Spaziphora Rondani, Dipt. Ital. Prodr., vol. 1. p. 99, 1856. 1 species. Type, Cordilura hydromyzina FALLEN.

Sphærina Wulp, Biol. Centr.-Amer., Dipt., vol. 2, p. 205, 1890. 1 species. Type, Sphrrina nitidula WuLP.

Sphrocera Latreille, Hist. Nat. Crust. et Ins., vol. 14, p. 394, 1805. 1 species. Type, Musca subsultans Fabricius (as curvipes, new species). Equals Cypsela Meigen, 1800.

$\checkmark$ Sphæromias Curtis, Brit. Ent., p. 285, 1829. 1 species. Type, Ceratopogon fasciatus Meigen (as albomarginatus, new species). Syn., Xylocrypta Kieffer, 1899.

Sphærophoria St. FArgeau and Serville, Encycl. Meth., vol. 10, pt. 2, p. 513, 1828. 7 species. Type, Musca scripta Linnsus, the third species, by designation of Rondani, Nuovi Ann. Sci. Nat. (Bologna), 1844, p. 458. Syn., Melithreptus Loew, 1840; Melitrophus Walker, 1856.

Sphageus Loew, Berliner Ent. Zeitschr., vol. 10, p. 32, 1866. 1 species. Type, Sphageus chalcoproctus LoEw.

$\checkmark$ Sphecomyia Latreille, Dict. Class. Hist. Nat., vol. 15, p. 545, 1829.1 species, without name. Type, Chrysotoxum vittatum Wienemans. Syn., Tyzenhausia Gorsk I, 1852.

Sphegina Meigen, Syst. Beschr. Zweifl. Ins., vol. 3, p. 193, 1822. 1 species (as 2). Type, Milesia clunipes Fallen.

Sphenoidoptera Williston, Biol. Centr.-Amer., Dipt., vol. 1, p. 295, 1901. 1 species. Type, Sphenoidoptera varipennis Williston.

Sphenometopa Townsend, Smiths. Misc. Coll., vol. 51, p. 64, 1908. 1 species. Type, Araba nebulosa Coquillett. Syn., Eumetopia Brauer and Bergenstamm, 1889, preoccupied.

$\checkmark$ Sphixea Rondani, Nuovi Ann. Sci. Nat. (Bologna) for 1844, p. 455, 1844. 1 species. Type, Musca semiluctifera Villiers (as Eristalis fulminans Fabricius).

Sphiximorpha Rondani, Ann. Soc. Ent. France for 1850, p. 212, 1850. 1 species. Type, Ceria subsessilis Illiger. Equals Cerioides Rondani, 1850.

Sphyracephala SAY, Amer. Ent., p. 116, 1828. 1 species. Type, Diopsis brevicornis SAY.

$\checkmark$ Sphyricera Lıox, Atti Inst. Veneto, ser. 3, vol. 9, p. 1336, 1864.2 species. Type, Echinomyia sphyricera MACQUART, the second species, by tautonymy. Equals Peleteria Desvoidy, 1830.

Sphyromyia Bigot, Bull. Soc. Ent. France for 1883, p. 108, 1883. 1 species. Type, Tachina robusta Wiedemann (as malleola, new species). Equals Peleteria Desvoidy, 1830 .

$\checkmark$ Sphyxosoma Rondani, Dipt. Ital. Prodr., vol. 1, p. 56,1856. 1 species. Type, Conops quadrifasciata De Geer. Equals Conops Linnzeus, 1758.

$\checkmark$ Spilochroa Williston, Journ. N. Y. Ent. Soc., vol. 15, p. 2, 1907. 1 species. Type, Heterochroa ornata JoHnson.

Spilogaster Macquart, Hist. Nat., Dipt., vol. 2, p. 293, 1835. 13 species. Type, Musca quadrum Fabricius, the seventh species, by designation of W EsTwood, Intr., vol. 2, Synops., 1840, p. 143. Equals Mydæa Desvordy, 1830.

Spilographa Loew, Monogr. Trypet., p. 39, 1862** 3 species. Type, Trypeta hamifera Loew, the second species, by present designation. Equals Euribia MeIGEN, 1800.

Spilomyia Meigen, Illiger's Mag., vol. 2, p. 273, 1803. 2 species. Type, Syrphus saltuum FABRICIUS (as diophthalmus FABRICIUS), the first pecies, by present designation. Syn., Milesia Latreille, 1805; Mixtemyia Macquart, 1834.

Spodius Loww, Berliner Ent. Zeitschr., vol. 2, p. 101, 1858. 1 species. Type, Spodius imbecillus LoEw. Equals Hesperinus WaLKER, 1848. 
Spoggosia Rondani, Dipt. Ital. Prodr., vol. 3, p. 182, 1859. 1 species. Type, Salia echinura Desvoidy (as occlusa, new species). Equals Phorocera Desvoidy, 1830.

$\checkmark$ Spogostylum Macquart, Dipt. Exot., vol. 2, pt. 1, p. 53, 1840. 1 species. Type, Spogostylum mystaceum MacQUaRT. Equals Anthrax ScopoLI, 1763.

$\checkmark$ Spylosia Rondani, Dipt. Ital. Prodr., vol. 1, p. 66, 1856. 1 species. Type, Tachina bisignata Meigen. Equals Meigenia Desvoidy, 1830.

$\checkmark$ Stachynia Macquart, Recueil Trav. Soc. Sci. Lille for 1834, p. 340, 1834. 1 species. Type, Myopa punctata Fabricius. Equals Dalmannia Desvoidr, 1830.

$\checkmark$ Stregeria Desvoldy, Hist. Nat. Dipt., vol. 1, p. 972, 1863. 1 species (as 22). Type, Guerinia festiva Desvorny. Equals Exorista Meigen, 1803.

$\checkmark \quad$ Stannia Rondanl, Dipt. Ital. Prodr., vol. 2, p. 14, 1857. Change of name for Ammobates Stannius, 1831, not of Latreille, 1809. Type, Ammobates notatus Stannius. Equals Tachytrechus HaLIDAY, 1851.

v Stearitia Lioy, Atti Inst. Veneto, ser. 3, vol. 9, p. 1105, 1864. 1 species. Type, Piophila fovenlata Meigen. Equals Piophila Fallen, 1810.

$\checkmark$ Stegana Meigen, Syst. Beschr. Zweifl. Ins., vol 6, p. 79, 1830. 2 species. Type, Musca coleoptrata Scopoli (as hypoleuca, new species), the second species, by designation of Westwood, Intr., vol. 2, Synops., 1840, p. 153 (as $S$. annulata Haliddy).

$\checkmark$ Stegomyia TheоваLD, in Howard's Mosquitoes, p. 234, June 1, 1901. 1 species. Type, Culex calopus Meigen (as fasciatus F ABricius).

$\checkmark$ Steleocheta Becker, Berliner Ent. Zeitschr., vol. 31, p. 129, 1887. 1 species. Type, Iteaphila macquarti ZetTerstedT, (as setacea, new species). Equals Iteaphila ZetTERSTEDT, 1838.

$\checkmark \quad$ Steneretma Loww, Monogr. Dipt. N. Amer., vol. 3, p. 186, 1873. 1 species. Type, Steneretma laticauda Loww.

Stenodexia WulP, Biol. Centr.-Amer., Dipt., vol. 2, p. 246, 1891. 1 species. Type, Stenodexia albicincta WULP.

$\checkmark \quad$ Stenogaster Lioy, Atti Inst. Veneto, ser. 3, vol. 9, p. 991, 1864. 1 species. Type, Chortophila angusta MACQUART. Equals Pegomya Desvoidy, 1830.

$\checkmark \quad$ Stenomacra LoEw, Monogr. Dipt. N. Amer., vol. 3, p. 180, 1873. 1 species. Type, Sepsis guerini Bigot. Not Stenomacra Stal, 1870. Equals Macrostenomyia Hendel, 1907.

$\checkmark$. Stenomicra Coquillett, Proc. U. S. Nat. Mus., vol. 22, p. 262, 1900.1 species. Type, Stenomicra angustata CoquilletT.

$\checkmark$ Stenomyia Loew, Berliner Ent. Zeitschr., vol. 11, p. 320, 1868. 1 species. Type, Stenomyia tenuis LoEw.

Stenopa Loew, Monogr. Dipt. N. Amer., vol. 3, p. 234, 1873. 1 species. Type, Trypeta vulnerata LoEw.

$\checkmark$ Stenopogon Loww, Linn. Ent., vol. 2, p. 453, 1847. 6 species. Type, Asilus sahaudus Fabricius, the last species, by designation of Coquilletr, Proc. Fnt. Soc. Washington, vol. 6, 1904, p. 179. Syn., Gonioscelis Schiner, 1866; Scleropogon LoEw, 1866.

Stenoporomyia Hendel, Wien. Ent. Zeit., vol. 10, p. 250, 1903. 1 species. Type, Milichia argyragastra Perris (as Lobioptera tiefi Mik). Equals Milichiella GigLioTos, 1895.

Stenoxenus Coquilletr, Ent. News, vol. 10, p. 60, 1899. 1 species. Type, Stenoxenus johnsoni CoQUILLETT.

$\checkmark$ Stephania Desvoidy, Hist. Nat. Dipt., vol. 1, p. 807, 1863.1 species. Type, Stephania meridionalis Désvoldy. Equals Wagneria Desvoidy, 1830.

Stethopathus W Andolleck, Zool. Jahrb. for 1898, p. 424, 1898. 1 species. Type, Puliciphora lucifera DAHL. Equals Puliciphora DAнL, 1897.

$\checkmark$ Stevenia Desvoidy, Essai Myod., p. 220, 1830. 2 species (as 4). Type, Stevenia tomentosa Desvoidy, the first species, by designation of Desvoidy, Hist. Nat. Dipt., vol. 2, 1863, p. 378. Syn., Hyperæa Desvordy, 1863; Trisonevra Lioy, 1864. 
Stibasoma Schrner, Verh. Zool.-Bot. Ges. Wien, vol. 17, p. 310, 1867. 1 species. Type, Tabanus theotirnia Wiedenann.

Stichopogon Loew, Linn. Ent., vol. 2, p. 499, 1847. 4 species. Type, Dasypogon elegantulus Wiedemann, the third species, by designation of Back, Trans. Amer. Ent. Soc., vol. 35, p. 332, 1909.

$\checkmark$ Stictocephala LoEw, Monogr. Dipt. N. Amer., vol. 3, p. 134, 1873. 4 species. Type, Ortalis vau $\mathrm{S}_{\mathrm{AY}}$, the first species, by original designation. Not Stictocephala $h$ Stal, 1869. Equals Pseudotephritis Johnson, 1902.

$\checkmark$ Stictodiplosis KiefFer, Bull. Soc. Ent. France for 1894, p. 28, 1894. 2 manuscript species, only the second ever described. Type, Stictodiplosis picridis KIEFFER. $\checkmark$ Stictomyia Bigot, Bull. Soc. Ent. France for 1885 , p. 166, 1885.1 species. Type, Stictomyia longicornis BIGot.

Stilbometopa Coquilletr, Can. Ent., vol. 31, p. 336, 1899. 1 species. Type, Ornithomyia fulvifrons WALKER.

Stilpnogaster Loew, Linn. Ent., vol. 4, p. 82, 1849. 1 species. Type, Asilus æmulus MEIGEN.

Stilpon Loew, Neue Beitr., vol. 6, p. 34, 1859. 2 species. Type, Tachydromia graminum Fallen, the first species, by designation of Coquillett, Proc. Ent. Soc. Washington, vol. 5, p. 257, 1903. Equals Agatachys Mergen, 1830.

Stomatodexia Brauer and Bergenstamm, Denkschr. Kais. Akad. Wiss. Wien, vol. 56, p. 125,1889 . 1 species. Type, Stomoxys cothurnata Wiedenann.

Stomoxoides Sch Type, Conops testacea Linneus. Syn., Myopa Fabricius, 1775; Pictinia Desvoidy, 1853; Myopella Desvordy, 1853, Phorosia Desvordy, 1853; Fairmairia Desvordy, 1853; Lonchopalpus Desvoidy, 1853; Purpurella Desvordy, 1853; Myopina Desvordy, 1853; Gonirhyncus Rondani, 1856; Ischiodonta Lioy, 1864.

Stomoxys Geoffrox, Hist. Abrégée Ins., vol. 2, p. 538, 1762. 1 species, without name. Type, Conops calcitrans Lrnnæus.

$\checkmark$ Stomphastica Loew, Berliner Ent. Zeitschr., vol. 8, p. 336, 1864. Change of name for Clusia Haliday, 1838, under the erroneous impression that the latter name is preoccupied in Botany. Type, Heteromyza flava Meigen. Equals Clusia Haliday, 1838.

Stonyx Osten Sacken, Biol. Centr.-Amer., Dipt., vol. 1, p. 94, 1886. 2 species. Type, Stonyx clelia Osten SACKEN, the second species, by present designation. Equals Villa Lroy, 1864.

Stratiomys Geofrroy, Hist. Abrégée Ins., vol. 2, p. 475, 1762. 8 species, without names. Type, Musca chamæleon Lrnswus, the first species, by designation of LATRĖille, Consider. Général., 1810, p. 442. Syn., Hoplomyia Zeller, 1842.

Strauzia Desvordy, Essai Myod., p. 718, 1830. 1 species (as 2). Type, Trypeta longipennis WIEDEMANN.

$\checkmark$ Strebla Wiedemann, Anal. Ent., p. 19, 1824.* 1 species. Type, Strebla avium Macquart (as Hippobosca vespertilionis Fabricius).

Stroblia Pokonny, Verh. Zool.-Bot. Ges. Wien, vol. 43, p. 541, 1893. Arbitrary change of name for Pseudolimnophora Strobl, 1893. Type, Musca triangula Falden. Equals Limnophora Desvoidy, 1830.

Sturmia Desvordy, Essai Myod., p. 171, 1830. 2 species (as 4). Type, Sturmia vanessæ Desvordy, the second species, by designation of Desvordy, Hist. Nat. Dipt., vol. 1, 1863, p. 888. Syn., Blepharipa Rondani, 1856; Lambertia Desvordy, 1863; Schaumia Desvordy, 1863; Belida Desvordy, 1863; Ugimyia Rondani, 1870; Ctenocnemis Kowarz, 1873; Argyrophylax Brauer and Bergenstamm, 1889; Crossocosmia Mik, 1890; Zygobothria Mrk, 1891; Xylotachina Brauer and Bergenstamm, 1891.

Stygeropis Loew, Berliner Ent. Zeitschr., vol. 7, p. 298, 1863. Arbitrary change of name for Prionocera Loew, 1844. Type, Prionocera pubescens Loew. Equals Prionocera LoEw, 1844.

Proc.N.M.vol.37-10-39 
Stylidir Westwood, Intr., vol. 2, Synops., p. 15t, 1840. 1 species. Type, Phthiridium biarticulatum Hernans. Equals Nycteribia Latreille, 1796.

$\checkmark$ Stylocomu Lioy, Atti Inst. Veneto, ser. 3, vol. 9, p. 1009, 1864. 1 species. Type, Sapromyza tulifer Melciex? Equals Sapromyza Fallex, 1810.

- Stylogaster Macquart, Hist. Nat., Dipt., vol. 2, p. 38, 1835. 1 species. Type, Comops stylata Fabricius. Syn., Stylomyia Westwood, 1850; Ptychoproctus Bigot, 1859.

$\checkmark$ Stylomyia Westwoon, Proc. Zool. Soc. Lond., vol. 18, p. 268, 1850. 2 species. Type, Stylomyiu leonum WEsTwoon, the first species, by present designation. Equals Stylogaster MACQUART, 1835.

- Styrex Scopoli, Ent. Carn., p. 367, 1763. 1 species. Type, Styrex holosericeus Scopolı. Syn., Chrysopilus Macquart, 1826.

$\checkmark$ Subclytia Pandelle, Rev. Ent., vol. 13, p. 96, 1894. 1 species. Type, Tachina rotundirentris Fallex. Equals Eliozeta Rondas1, 1856.

Subula Meigen, Syst. Beschr. Zweifl. Ins., vol. 2, p. 14, 1820. 1 species. Type, Tylophagus maculatus Meigen. Not Subula Schumacher, 1817. Equals Solva W ALKER, 1860.

$\checkmark$ Suillia Desvoldy, Essai Myod., p. 642, 1830. 12 species. Type, Heleomyza rufa FALLex (as fungorum, new species), the second species, by present designation. Syn., Herbina Desvoldy, 1830.

$\checkmark$ Sylria Desvoldy, Essai Myod., p. 636, 1830. 5 species. Type, Sylria apicalis Desvoldy, the first species, by present designation. Equals Sapromyza FAllex, 1810.

Sylvicolæ Harris, Exp. Engl. Ins., p. 100, 1776. 16 species. Type, Tipula fenestrulis Scopoli (as brevis, new species), the thirteenth species, by present designation. Syn., Phryne Meigen, 1800; Anisopus Meigen, 1803; Ihlyphus Latreille, 1805 .

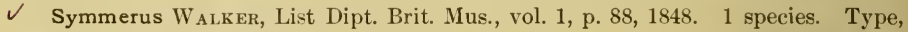
Mycetobia annulata MEIGex (as ferrugineus, new species). Syn., Plesiastina WINNERTZ, 1852.

N Symphoromyia Frauenfeld, Verh. Zool.-Bot. Ges. Wien, vol. 17, p. 496, 1867. 1 species. Type, Atherix melixna Meigen.

$\checkmark$ Symplecta Migigex, Syst. Beschr. Zweifl. Ins., vol. 6, p. 282, 1830. 2 species. Type, Limonia hybrida MElgen, the first species, by designation of Westwood, Intr., vol. 2, Synops., 1840, p. 128. Syn., Helobia St. Fargeau and Serville, 1828, preoccupied; Idioneura PHıLIPPı, 1865; Symplectomorpha MIK, 1886.

$\checkmark$ Symplectomorpha $\mathrm{I}_{1 \mathrm{~K}}$, Wien. Ent. Zeit., vol. 5, p. 318, 1886. 2 species. Type, Limnobia stictica Melgex, the first species, by original designation. Equals Symplecta MeigeN, 1830.

$\checkmark$ Sympycnus Loew, Neue Beitr., vol. 5, p. 42, 1857. 5 species. Type, I'orphyrops annulipes MeIGEN, the first species, by present designation.

$\checkmark \quad$ Synarthrus Loew, Nene Beitr., vol. 5, p. 35, 1857. 1 species. Type, IIydrochus tarsatus Fallex. Equals Syntormon Loew, 1857.

$\checkmark \quad$ Syndyas Loew, Öfv. Kongl. Vet. Akad. Förh., vol. 14, p. 369, 1857. 2 species. Type, Syndyas opaca Loew, the first species, by designation of Conullletr, Proc. Ent. Soc. Washington, vol. 5, 1903, p. 257.

$\checkmark$ Syneches WALker, Ins. Saund., Dipt., p. 165, 1852. 1 species. Type, syneches simplex Walker. Equals Acromyia Latrellle, 1809.

$\checkmark \quad$ Syneura Brues, Trans. Amer. Ent. Soc., vol. 29, p. 383, 1903. 1 species. Type, Phoca cucciphila CoQunlett.

$\checkmark$ Syntemna Winnertz, Verh. Zool.-Bot. Ges. Wien, vol. 13, p. 767, 1863.1 species. Type, syntemna morosa W INNERTz.

$\checkmark$ Synthesiomyia Brauer and Bergenstamn, Denkschr. Kais. Akad. Wiss. Wien, vol. 60, p 96, 1893. 1 species. Type, Synthesiomyia brasiliana Brater and Bergengtami. 
Syntormon LoEw, Neue Beitr., vol. 5, p. 35, 1857. 5 species. Type, Rhaphium metathesis Loew, the first species, by present designation. Syn., Plectropus Haliday, 1831, preoccupied; Synarthrus Loww, 1857.

Syritta_ts. Fargeau and Serville, Encycl. Meth., vol. 10, pt. 2, p. 808, 1828. 1 species. Type, Musca pipiens Linnwus. Syn., Coprina Zetterstedt, 1837; Planes RoNDANi, 1864.

Syrphus Fabricius, Syst. Ent., p. 762, 1775. 49 species. Type, Musca lucorum Linn.eus, the nineteenth species, by designation of Curtis, Brit. Ent., 1839, p. 753. Syn., Leucozona Schiner, 1860. (Syrphus of authors equals Epistrophe WaLker.)

Systæchus Loew, Neue Beitr., vol. 3, p. 34, 1855. 14 species (as 15). Type, Bombylius sulphureus MiKan, the sixth species, by present designation.

Systropus IViedemann, Nova Dipt. Gen., p. 18, 1820.* 1 species. Type, Systropus macilentus Wiedemann. Syn., Cephenus Berthold, 1827.

Tabanus Linnæus, Syst. Natur., 10th ed., p. 601, 1758. 12 species. Type, Tabanus bovinus Linnzes, the first species, by designation of Latreille, Consider. Général., 1810, p. 443. Syn., Therioplectes Zeller, 1842; Brachystomus Costa, 1857; Agelanius Rondani, 1864; Atylotus Osten Sacken, 1876.'

Tabuda W WALKER.

Tachina Meigen, Illiger's Mag., vol. 2, p. 280, 1803. 3 species. Type, Musca grossa LinNeus, the first species, by designation of WAChTL, Wien. Ent. Zeit., vol. 13, 1894, p. 142. Equals Larvævora Meigen, 1800. (Tachina of authors equals Exorista Meigen.)

$\checkmark$ Tachinodes Brauer and Bergenstamm, Denkschr. Kais. Akad. Wiss. Wien, vol. 56, p. 133, 1889. 1 species. Type, Musca hystrix Fabricius. Equals Archytas $\mathrm{J}_{\text {ÆNnICKE, }}$ 1867.

Tachinomyia Townsend, Trans. Amer. Ent. Soc., vol. 19, p. 96, 1892. 1 species. Type, Tachinomyia robusta Townsend. Equals Exorista Meigen, 1803.

Tachinophyto Townsend, Trans. Amer. Ent. Soc., vol. 19, p. 130, 1892. 1 species. Type, Tachinophyto floridensis Townsend. Syn., Pseudomyothyria Townsend, 1892; Methypostena Townsend, 1908; Lixophaga Townsend, 1908.

Tachinopsis Coquillett, Rev. Tach., p. 38, 1897. 1 species. Type, Tachinopsis mentalis CoquilletT.

Tachista Loew, Zeitschr. Ent. Breslau, vol. 14, p. 7,1860. 11 species. Type, Tachydromia connexa Meigen, the sixth species, by designation of Coquillett, Proc. Ent. Soc. Washington, vol., 5, 1903, p. 258. Equals Coryneta Meigen, 1800.

Tachydromia Meigen, Illiger's Mag., vol. 2, p. 269.1803. 2 species. Type, Tachydromyia connexa MeIGEN (as cimicoides F ABricius), the second species, by designation of Coquillett, Proc. Ent. Soc. Washington, vol. 5, 1903, p. 258. Equals Coryneta Meigen, 1800.

Tachypeza Meigen, Syst. Beschr. Zweifl. Ins., vol.6, p. 341, 1830. 10 species. Type, Tachydromia nubila Meigen, the eighth species, by designation of Rondani, Dipt. Ital. Prodr., vol. 1, 1856, p. 147.

Tachytrechus HaLiday, in Walker's Ins. Britt., Dipt., vol. 1, p. 173, 1851 . Change of name for Ammobates Stannius, 1831, not of Latreille, 1809. Type, Ammobates notatus Stannius. Syn., Ammobates Stannius, 1831, preoccupied; Stannia Rondani, 1857; Macellocerus Мıк, 1878.

Tacina Walker, Ins. Saund., Dipt., p. 9, 1850. 3 species. Type, Pangonius micans Meigen, the first species, by present designation. Equals Pangonius Latreille, 1802. $\checkmark$ Trehobates Haliday, Zool. Journ., vol. 5, p. 358, 1831. 2 species (as 3). Type, Hydrophorus juculus $\mathrm{F}_{\text {Allen, }}$ the first species, by present designation. Equals Medetera FISCHER, 1819. 
Tæniorhynchus Arribalzaga, Rev. Mus. La Plata, vol. 1, p. 389, 1891. 3 species. Type, Culex titllans WaLKer (as tioniorhynchus Wiedemaxi), the first species, by tautonymy. Syn., Panoplites Theobald, 1900; Mansonia Blancinard, 1901.

$\checkmark$ Talmonia Desvoidy, Hist. Nat. Dipt., vol. 1, p. 704, 1863. 1 species. Type, Tulmonia tibirlis Desvordy. Equals Ceranthia Desvoldy, 1830.

$\checkmark$ Tuniporlu Rondani, Dipt. Ital. Prodr., vol. 1, p. 116, 1856.1 species. Type, Calobata calceata Fallen. Equals Trepidaria Meigen, 1800.

$\checkmark$ Tanyglossa Meigex, Illiger's Mag., vol. 2, p. 267, 1803. 1 species. Type, Tabanus naauritanus Linneus (as haustellatus Fabricius). Equals Pangonius Latreille, 1802.

$\checkmark$ Tanypeza Fallen, Opom. Svecie., p. 4, 1820. 1. species. Type, Trnypezu longimana FALLEN.

$\checkmark$ Tanypremna Osten SAcken, Biol. Centr.-Amer., Dipt., vol. 1, p. 19, 1886.1 species. Type, Tanypremna opilio Osten SACKen.

$\checkmark$ Tanyptera Latreille, Hist. Nat. Crust. et Ins., vol. 14, p. 286, 1805. 1 species. Type, Tipula atrata LivNeus. Equals Flabellifera MeIGev, 1800.

$\checkmark$ Tanypus MeIGen, Illiger's Mag., vol. 2, p. 261, 1803. 2 species. Type, Tipula cincta Fabricius, the first species, by designation of Latreille, Consider. Général., 1810, p. 442. Equals Pelopia MeIgen, 1800.

$\checkmark$ Tanytarsus WulP, Tijdschr. Ent., vol. 17, p. 134, 1874. 12 species. Type, Chironomus punctipes $\mathrm{W}_{1 \mathrm{EDEMANN}}$, the first species, by present designation.

$\checkmark$ Taphrosia Rondani, Dipt. Ital. Prodr., vol. 1, p. 182, 1856. 1 species. Type, Limnobia tenella Meigen. Equals Gonomyia Meigen, 1818.

- Tapinocera Macquart, Dipt. Exot., vol. 1, pt. 2, p. 78, 1838. 1 species. Type, Laphria brevicomis Wiedemann. Equals Apiocera Westwood, 1835.

Taracticus Loew, Berliner Ent. Zeitschr., vol. 16, p. 240, 1872. 1 species. Type, Dioctria octopunctata SAY.

Tauromyia GifiLlo-Tos, Boll. Mus. Zool. Univ. Torino., vol. 8, no. 158, p. 8, 1893. 1 species. Type, Tauromyia pachyneura Giglio-Tos.

Taxigramma Perris, Ann. Soc. Linn. Lyons for 1850, p. 209, 1852. 1 species. Type, Miltogrammu heteroneura Meigen (as pipiens, new species). Syn., Heteropterinu Macquart, 1854; Metopilla Rondan1, 1859; Elpigia Desvoidy, 1863.

$\checkmark$ Telejoneura Rondani, Arch. Zool. Modena, vol. 3, p. 48, 186t. Change of name for Trupanea Macquart, 1838, not of Schrank, 1803. Type, Asilus maculatus Fabricius. Equals Bactria MeIGeN, 1820.

- Telmatobia Stenhammar, Kongl. Vet. Akad. Handl. for 1843, p. 149, 18t4. 1 species. Type, Notiplita renea Fallen. Equals Pelina Haliday, 1839.

$\checkmark$ Telmatogeton Schiner, Verh. Zool.-Bot. Ges. Wien, vol. 16, p. 931, 1866. 1 species. Type, Telmatogeton sanctipauli ScH1Ner.

$\checkmark$ Telothyria Wulp, Biol. Centr.-Amer., Dipt., vol.2, p. 167, 1890. 38 specics. Type, Telothyria cupreiventris WULP, the thirtieth species, by designation of BRAUER and Bergenstamm, Denkschr. Kais. Akad. Wiss. Wien, vol. 60, 1893, p. 132.

$\checkmark$ Temuocera St. Fargeau and Serville, Encycl. Meth., vol. 10, pt. 2, p. 786, 1828. 1 species. Type, Temnocera violacea St. Fargeau and Serville. Equals Volucella Geofrroy, 1762.

- Temnostoma St. Fargeau and Serville, Encycl. Meth., vol. 10, pt. 2, p. 518, 1828. 4 species. Type, Ifilesia bombylans Fabricius, the second species, by present designation.

Tendipes MeIgen, Nouv. Class. Mouch., p. 17, 1800.* No species. Chironomus Meigen, 1803, is a change of name. Type, Tipula plumosa Lisnæus. Ayn., Clironomus Melgen, 1803.

$\checkmark$ Tenuicera Pindelle, Rev. Ent., vol. 15, p. 32, 1896. 1 spccies. Type, Tuclina incana Fallen (as oxyrhina, new species). Equals Meigenia Desvoidy, 1830. 
Tephritis Latreille, Hist. Nat. Crust. et Ins., vol. 14, p. 389, 1805.1 species. Type, Musca solstitialis Linnæus. Syn., Urophora Desvoldy, 1830. (Tephritis of authors equals Spathulina Ronda N1.)

Tephrochlamys Loew, Zeitschr. Ent. Breslau, vol. 13, p. 72, 1862. 5 species. Type, Heleomyza canescens Melgen (as rufiventris Meigen), the fourth species, by present designation. Equals Lentiphora Desvoldy, 1830.

$\checkmark$. Tephronota Loew, Zeitschr. Ges. Nat., vol. 32, p. 6, 1868. 3 species. Type, (rrtalis gyrans Loew, the second species, by designation of Loww, Monogr. Dipt. N. Amer., vol. 3,1873, p. 57.

Terellia Desvoldy, Essai Myod., p. 758, 1830. 1 species (as 2). Type, Musca serratulie Linneus. Syn., Sitarea Desvoldy, 1830; Carpomya Rondan1, 1856; Cerajocera Rondan1, 1856.

Terelliosoma Rondan1, Dipt. Ital. Prodr., vol. 1, p. 109, 1856. 1 species. Type, Tetanops impunctata LoEw (as heryngii, new species). Equals Tetanops Fallen, 1820.

レ'Teremyia Macquart, Hist. Nat., Dipt., vol. 2, p. 514, 1835. 1 species. Type, Lonchæa laticornis Mergen. Equals Lonchæa Fallen, 1820.

Terenia Desvoldy, Essai Myod., p. 640, 1830. 4 species. Type, Terenia suillorum Desvolny, the first species, by present designation. Equals Sapromyza Fallen, 1810.

Tersesthes Townsend, Psyche, vol. 6, p. 370, 1893. 1 species. Type, Tersesthes torrens Townsend.

Tessaroch̆cta BEzz1, Zeitschr. Hymen. Dipt., vol. 6, p. 50, 1906 . Change of name for Tetrachrta Brauerand Bergenstami, 1894, preoccupied. Type, Tetracheta obscura Brauer and Bergenstamm. Equals Peleteria Desvoidy, 1830.

Tetanocera Latreille, Hist. Nat. Crust. et Ins., vol. 14, p. 385, 1805. 2 species. Ty pe, Musca coryleti Scopol1, the first species, by present designation. Syn., Pherbina Desvoldy, 1830.

Tetanops Fallen, Ortal. Sveciæ, p. 2, 1820. 1 species. Type, Tetanops myopina Fallen. Syn., Terelliosoma Rondan1, 1856.

$\checkmark$ Tetrachata Brauer and Bergenstamm, Denkschr. Kais. Akad. Wiss. Wien, vol. 61, p. 611, 1894. 1 species. Type, Tetrachrta obscura Brauer and Bergenstamm. Equals Peleteria Desvoidy, 1830.

Tetrachæta Stein, Berliner Ent. Zeitschr., vol. 42, p. 254, 1898. 1 species. Type, Tetrachrta unica Stein. Not Tetrachæta Ehrenberg, 1844. Equals Tetramerinx BERG, 1898.

Tetradiscus B1Got, Ann. Soc. Ent. France for 1886, p. 370, 1886. 2 species. Type, Tetradiscus piclus BıGoт, the first species, by present designation.

Tetragoneura WinnerTz, Ent. Zeit. Stettin, vol. 7, p. 18, 1846. 2 species. Type, Sciophila sylvatica Curtis (as distincta, new species), the second species, by designation of Johannsen, Gen. Ins., Dipt., Mycet., 1909, p. 34.

Tetragrapha Brauer and Bergenstamm, Denkschr. Kais. Akad. Wiss. Wien, vol. 58, p. 351, 1891.1 species. Type, Tetragrapha tessellata Brauer and Bergenstamm. Tetramerinx Berg, Com. Mus. Nac. Buenos Aires, vol. 1, p. 17, 1898 . Change of name for Tetrachæta Stein, 1898, not of Ehrenberg, 1844. Type, Tetrachæta unica Stein. Syn., Tetrachate Stein, 1898, preoccupied.

Tetropismenus Loew, Zeitschr. Ges. Nat., vol. 48, p. 333, 1876. 1 species. Type, Tetropismenus hirtus LoEw.

Teuchocnemus Osten SAcken, Büll. Buff. Soc. Nat. Sci., vol. 3, p. 58, 1876. 2 species. Type, Pterallastes lituratus Loew, the second species, by designation of Wrlliston, Synops. N. Amer. Syrph., 1886, p. 199.

Teucholabis Osten Sacken, Proc. Acad. Nat. Sci. Phila. for 1859, p. 222, 1860. 1 species. Type, Teucholabis complexa Osten Sacken.

Teuchophorus Loew, Neue Beitr., vol. 5, p. 44, 1857. 2 species. Type, Dolichopus spinigerellus ZETTERSTEDT, the first species, by present designation. 
$\checkmark$ Thalassomya Schiner, Verh. Zool.-Bot. Ges. Wien, vol. 6, J. 219, 1856.1 species. Type, Thalassomya frauenfeldi ScHıNer.

$\checkmark$ Thamnodromia Mik, Wien. Ent. Zeit., vol. 5, p, 278, 1886. Change of name for Phyllodromia Zetterstedt, 1837, not of Serville, 1839. Type, Empis melanocephala Fabricius. Equals Hemerodromia Melgen, 1822.

$\checkmark$ Thapsia Desvoldy, Hist. Nat. Dipt., vol. 1, p. 689, 1863. 1 species. Type, Tarhina albicollis Melgen. Equals Neæra Desvoldy, 1830.

$\checkmark$ Thaumalea Ruthe, Isis von Oken for 1831, p. 1211, Nov., 1831. 1 species. Type, Orphnephila devia HAL1DAY (as testacea, new species). Equals Orphnephila HaL11)AY, September, 1831.

$\checkmark \quad$ Thecophora Roxdanl, Nuovi Ann. Sci. Nat. (Bologna) for 1845, p. 15, 1845.1 species. Type, Myopa atra Fabricius. Syn., Occemya Desvoiny, 1853.

$\checkmark$ Thelairia Desvoldy, Essai Myod., p. 214, 1830. 1 species (as 2). Type, Muscu nigripes FABRlCıUs.

$\checkmark$ Thelairodes Wulp, Biol. Centr.-Amer., Dipt., vol. 2, p. 254, 1891. 3 speciea. Type, Homodexia vittigera Blgot, the first species, by designation of Brauer and Bergenstamu, Denkschr. Kais. Akad. Wiss. Wien, vol. 58, 1891, p. 377.

- Themira Desvoldy, Essai Myod., p. 745, 1830. 2 species. Type, Themira pilosa Desvoldy, the first species, by present designation. Syn., Cheligaster MacQuaRT, 1835; Hriliclaya Rondan1, 1856.

Theobaldia Neveu-Lemaire, Compt. Rend. Soc. Biol., vol. 54, p. 1331, 1902.1 species. Type, Culex annulatus Schrank. Syn., Theobaldinella Blanchard, 1905.

7 Theobaldinella BrancharD, Les Moust., p. 390, 1905. Change of name for Theobaldia Neveu-Lema1Re, 1902, under the mistaken impression that it interferes with Theobaldius Neville. Type, Culex annulatus Schraxk. Equals Theobaldia NevetLEMAIRE, 1902.

- $\checkmark$ Theone Desvoldy, Hist. Nat. Dipt., vol. 2, p. 401, 1863.1 species (as 2). Type, Tuchina polyodon Meigen. Equals Amobia Desvoldy, 1830.

$\checkmark$ Theresia Desvoldy, Essai Myod., p. 325, 1830. 1 species. Type, Theresia tandrec Desvoldy. Syn., Sardiocera Brauer and Bergenstamm, 1889.

$\checkmark$ Thereva Latreille, Precis, p. 167, 1796. No species. In his Hist. Nat. Crust. et Ins., vol. 3, 1802, p. 441, 1 species. Type, Musca plebeja Linnæus. Syn., Dialineura RoNDAN1, 1856.

Therioplectes Zeller, Isis von Oken for 1842, p. 819, 1842. 8 species. Type, Tabamus tricolor ZELLER, the first species, by present designation. Equals Tabanus LinNæus, 1758.

- Thevenemyia Bigot, Bull. Soc. Ent. France for 1875, p. 174, 1875.1 species. Type, Therenemyia califormica Biciot. Equals Eclimus LoEw, 1844.

$\checkmark$ Thinodromia Melander, Ent. News, vol. 17, p. 370, 1906. 1 species. Type, Thinodromia inchoata MeLANDER.

$\checkmark$ Thinophilus Wahlberg, Öfv. Kongl. Vet. Akad. Förh., vol. 1, p. 37, 1844. 2 species. Type, Rhaphium ,flavipalpe ZETTERSTEDT, the first species, by designation of ZETTERstEDT, Dipt. Scand., vol. 8, 1849, p. 3101.

Thricops Rondanı, Dipt. Ital. Prodr., vol. 1, p. 96, 1856. 1 species. Type, Aricia hirtula Zetterstedt. Syn., Tricophthicus Rondanl, 1861; Rhynchotrichops ScHNaBL, 1889.

$\checkmark$ Thrycolyga Rondani, Dipt. Ital. Prodr., vol. 1, p. 68, 1856. 1 species. Type, Thrycolyga nova Rondan. Syn., icotia Desvoldy, 1863.

$\checkmark \quad$ Thrypticus Gerstecker, Ent. Zeit. Stettin, vol. 24, P. 43, 1864. 1 species. Type, Thrypticus smaragdinus Gerstecker. Syn., Aphantotimus Wheeler, 1890; Zunthotricha A LDR1CH, 1896.

$\checkmark \quad$ Thryptocera Macquart, Recueil Trav. Soc. Sci. Lille for 1834, p. 310, 1834.9 species. Type, Tachina pilipennis Fallen, the ninth species, by designation of RoN- 
DANI, Dipt. Ital. Prodr., vol. 1, 1856, p. 60. Equals Actia Desvordy, 1830. (Thryptocera of authors equals Ceranthia Desvorny.)

Thyridanthrax Osten Sacken, Biol. Centr.-Amer., Dipt., vol. 1, p. 123, 1886. 4 species Type, Anthrax selene Osten SAcken, the first spécies, by present designation. Equals Villa Lioy, 1864.

- Thyrimyza ZetTersteDt, .Dipt. Scand., vol. 6, p. 2336, 1847. 9 species. Type, Musca flava Linn.æus, the first species, by designation of Rondani, Bull. Soc. Ent. Ital., vol. 6, 1874, p. 244 . Equals Chyromya Desvoidy, 1830.

Thysanomyia Brauer and Bergenstamm, Denkschr. Kais. Akad. Wiss. Wien, vol. 58, p. 340, 1891. 1 species. Type, Brachicoma fimbriata WulP.

$\checkmark$ Tinearia Schellenberg, Genres Mouches Dipt., pl. 40, figs. 1, a, b, c, and 2, b, d, 1803. 2 species. Type, Psychoda alternata SAY, the first species, by present designation. Equals Psychoda Latreille, 1796.

Tinolestes Coquillett, Proc. Ent. Soc. Washington, vol. 7, p. 185, 1906.1 species. Type, Tinolestes latisquama Coquillett.

Tipula Linnæus, Syst. Nat., 10th ed., p. 585, 1758. 37 species. Type, Tipula olerucea Linnaus, the fourth species, by designation of Latreille, Consider. Général., 1810, p. 442. Syn., Anomaloptera Lioy, 1864; Oreomyza Pokorny, 1887.

Titania MeIgev, Nouv. Class. Mouch., p. 35, 1800.* No species. Chlorops Meigen, 1803 , is a change of name. Type, Chlorops leta Meigen. Syn., Chlorops Meigen, 1803; Oscinis Latreille, 1804; Cotilea Lioy, 1864.

Tolmerus Loew, Linn. Ent., vol. 4, p. 94, 1849. 4 species. Type, Asilus pyragra ZELLER, the fourth species, by present designation.

Tomoplagia, new name. Type, Trypeta obliqua SAy. Syn., Plagiotoma Loew, preoccupied.

Toreus Melander, Ent. News, vol. 17, p. 376, 1906.1 species. Type, Empis neomexicana MELANDER.

Townsendia Williston, Kansas Univ. Quart., vol. 4, p. 107, 1895. 1 species. Type, Tounsendia minuta Williston.

Toxomerus Macquart, Dipt. Exot., Suppl. 5, p. 93, 1855. 1 species. Type, Scæra geminata SAY (as notatus, new species). Syn., Mesogramma Loew, 1865; Mesograpta LoEw, 1872.

Toxonevra Macquart, Hist: Nat., Dipt., vol. 2, p. 404, 1835. 1 species. Type, Musca pulchella Rossi (as fasciata, new species). Equals Palloptera Fallen, 1820.

Toxophora Meigen, Illiger's Mag., vol. 2, p. 270, 1803. No species. In his Klass. Beschr. Zweifl. Ins., 1804, p. 273, 1 species. Type, Asilus maculatus Rossi.

- Toxorhina Loew, Linn. Ent., vol. 5, p. 400, 1851. 3 fossil and 1 recent species. Type, Toxorhina fragilis Loew, the recent species, by designation of OsTEN SACKen, Proc. Acad. Nat. Sci. Phila. for 1861, p. 230.

Toxotrypana GERSTæcker, Ent. Zeit. Stettin, vol. 21, p. 191, 1860. 1 species. Type, Toxotrypana curvicauda Gerstecker. Syn., Mikimyia Bigot, 1884.

- Traginops Coqulletet, Ent. News, vol. 11, p. 429, 1900. 1 species. Type, Traginops irrorata CoQuilletT.

Trennia Desvoldy, Essai Myod., p. 484, 1830. 1 species. Type, Anthomyia errans Meigen (as nigricornis, new species). Equals Phaonia Desvoldy, 1830.

Trepidaria Meigen, Nouv. Class. Mouches, p. 35, 1800.* No species. Calobata Meigen, 1803, is a change of name. Type, Musca petronella Linnsus. Syn., Calobata Melgen, 1803; Neria Desvoidy, 1830; Rainieria Rovdsnl, 1843; Tanipoda RonDANI, 1856.

$\checkmark$ Trepophrys Townsend, Smiths. Misc. Coll., vol. 51, p. 95, 1908. 1 spècies. Type, Trepophrys cinerea Townsend. Equals Pseudochæta Coquillett, 1895.

$\checkmark$ Triachora Townsend, Smiths. Misc. Coll., vol. 51, p. 105, 1908. 1 species. Type, Latreillia unifasciata Desvordy. Equals Goniomima Townsend, 1908. 
Trichiopoda Latreille, in Cuvier's Regne Anim., new ed., vol. 5, p. 512, 1829. 2 species. Type, Thereva plumipes Fabricius, the first species, by present designation. Syn., Iomogenia WulP, 1892; Trichopododes Townsend, 1893; Pennapoda Townsend, 1897; Eutrichopoda Townsend, 1908; Galactomyia Townsend, 1908; Polistomyia Townsend, 1908; Euomogenia Townsend, 1908.

$\checkmark$ Trichobius Gervais, Atlas Zool., p. 14, 1844. 1 species. Type, Trichobius parasiticus GervaIs.

$\checkmark \quad$ Trichocerd MeIgen, Illiger's Mag., vol. 2, p. 262, 1803. 1 species. Type, Tipula hiemalis De Geer. Equals Petaurista Meigen, 1800.

$\checkmark \quad$ Trichomyia Curtis, Brit. Ent., p. 745, 1839. 1 species. Type, Trichomyia urbica CurTis.

Trichonta Winnertz, Verh. Zool.-Bot. Ges. Wien, vol. 13, p. 798, 1863. 7 speries. Type, Mycetophila melanura STEGER, the first species, by designation of JoHAnnsex, Gen. Ins., Dipt., Mycet., 1909. p. 94.

Trichophora Macquart, Dipt. Exot., Suppl. 2, p. 62, 1847. 1 species. Type, Trichophora nigra Macquart. Syn., Siphoniomyia Bigot, 1885; Paragymnomma Brauer and Bergenstamu, 1891.

$\checkmark$ Trichopododes Townsend, Can. Ent., vol. 25, p. 166, 1893 . Change of name for Homogenia WuLP, under the mistaken impression that it is preoccupied by Homogenes Trouson. Type, Homogenia rufipes Wulp. Equals Trichiopoda Latreille, 1829.

$\checkmark$ Trichoprosopon Theobald, Journ. Trop. Med., vol. 4, p. 235, July 15, 1901 . No species. In his Monogr. Culic., vol. 2, November 23, 1901, p. 285, 1 species. Type, Trichoprosopon niripes Theobald. Syn., Joblotia Blanchard, December 6, 1901.

$\checkmark$ Trichoptera Meigen, Illiger's Mag., vol. 2, p. 261, 1803. 2 species. Type, Psychoda alternata $\mathrm{SAY}_{\mathrm{Y}}$ (as Tipula phalionoides FABriciUs), the first species, by present designation. Equals Psychoda Latreille, 1796.

$\checkmark$ Trichoptera Lioy, Atti Inst. Veneto, ser. 3, vol. 9, p. 1104, 1864. 2 species. Type, Diastata adusta Meigex, the first species, by present designation. Not Trichoptera Meigen, 1803. Equals Calopterella, new name.

$\checkmark$ Trichopteromyia Williston, Trans. Ent. Soc. Lond. for 1896, p. 255, 1896.1 species. Type Trichopteromyia modesta Williston.

$\checkmark$ Trichosia Winnertz, Beitr. Monogr. Sciarinen, p. 173, 1867. 3 species. Type, Trichosia splendens WINNERTz, the first species, by present designation.

$\checkmark$ Trichotanypus Kieffer, Ann. Soc. Sci. Bruxelles, vol. 30, p. 319, 1906. No species. In 1906, 1 species. Type, Tanypus posticalis LunDBeck.

$\checkmark$ Triclis Loew, Bemerk. Asiliden, p. 17, 1851. 1 species. Type, Dasypogon oliraceus Loew. Syn., Gastrichelius Rondani, 1856.

$\checkmark$ Tricogena Rondanı, Dipt. Ital. Prodr., vol.1, p. 88, 1856. 1 species. Type, Tachina rubricosa Meigen (as truquï, new species). Syn., Frauenfeldia Egger, 1865.

$\checkmark$ Tricophthicus Rondani, Dipt. Ital. Prodr., vol. 4, p. 9, 1861 . Change of name for Thricops Rondani, 1856, under the mistaken impression that it is preoccupied by Trichops Mannerheim, 1846 (?). Type, Aricia hirtula Zetterstedt. Equals Thricops Rondani, 1856.

$\checkmark$ Tricyphona ZetTerstedt, Isis von Oken for 1837, p. $65,1837.1$ species. Type, Limonia immaculata Meigen. Syn., Amalopis Haliday, 1856; Bophrosia Rondani, 1856; Crunobia Kolenati, 1860.

$\checkmark$ Trigonometopus Macquart, Hist. Nat., Dipt., vol. 2, p. 419, 1835. 1 species. Type, Tetanocera frontalis Meigen. Syn., Oxyrhina Meigen, 1838.

$\checkmark$ Trigonostomi Lior, Atti Inst. Veneto, ser. 3, vol. 9, p. 990, 1864. 1 species. Type, Chortophila frontalis Macquart. Equals Pegomya Desvoidy, 1830.

$\checkmark$ Trimicra Osten Sacken, Proc. Acad. Nat. Sci. Phila. for 1861, p. 290, 1862. 1 species. Type, Trimicra anomala Osten Sacken. 
Trinaria Muls.nt, Mem. Acad. Lyons for 1852, p. 18, 1852.* 2 species. Type, Anthrax rutila Wiedemann, the first species (as interrupta, new species), by present designation. Equals Mima Meigen, 1820.

Tineura MeIgen, Illiger's Mag., vol. 2, p. 276, 1803. No species. In his Klass. Beschr. Zweifl. Ins., 1804, pp. 313, 314, 4 species. Type, Musca aterrima Fabricius, the first species (as atra, new species), by designation of Brues, Genera Insect., Dipt., Phoridie, p. 8, $1906 . \quad$ Equals Phora Latreille, 1796.

Triodites Osten Sacken, Bull. 3, U.S. Geol. Surv.,p. 245, 1877. 1 species. Type, Triodites mus Osten Sacken. Equals Aphœbantus Loew, 1872.

Triodonta Williston, Bull. Brooklyn Ent. Soc., vol. 7, p. 136, 1885. Change of name for Polydonta Macquart, 1850, not of Fischer, 1807. Type, Merodon curvipes Wiedemann. Not Triodonta Bory, 1824. Equals Polydontomyia Williston, 1896.

Triogma Schiner, Wien. Ent. Monatschr., vol. 7, p. 223, 1863.1 species. Type, Limnobia trisulcata Schummel.

Triplasius Loew, Neue Beitr., vol. 3, p. 7, 1855. 1 species. Type, Bomby ius bivittatus LoEw.

Triptotricha LoEw, Berliner Ent. Zeitschr., vol. 16, p. 235, 1872. 1 species. Type, Triptotricha lauta LoEw.

Trisometopia Lroy, Atti Inst. Veneto, ser..3, vol. 10, p. 77, 1864. 1 species. Type, Trisometopia thoracica Lior. Equals Megaselia Rondani, 1856.

Trisonevra Lioy, Atti Inst. Veneto, ser. 3, vol. 10, p. 68, 1864. 1 species. Type, Dexia melania Meigen (as Ptilocera cilipennis Macquart). Equals Stevenia DesvoIDY, 1830.

Tritochxta Brauer and Bergenstamm, Denkschr. Kais. Akad. Wiss. Wien, vol. 56, p. 92, 1889. 1 species. Type, Tachina pullata Meigen (as prosopoides, new species). Equals Zenilla Desvordy, 1830.

Tritonia Meigen, Nouv. Class. Mouches, p. 33, 1800.* No species. Spilomyia Meigen, 1803, is a change of name. Type, Musca vespiformis Linneus. Not Tritonia Cuvier, 1798. Equals Spilomyia Meigen, 1803.

Tritoxa LoEw, Monogr. Dipt. N. Amer., vol. 3, p. 102, 1873. 3 species. Type, Trypeta flexa Wiedemann, the first species, by present designation.

ᄂ. Trixa Mergex, Syst. Beschr. Zweifl. Ins., vol. 4, p. 222, 1824. 6 species. Type, Trixa dorsalis Meigen, the fifth species, by designation of Westwoon, Intr., vol. 2, Synops., 1840, p. 138. Syn., Murana Meigen, 1824; Crameria Desvordy, 1830; Semiomyia Macquart, 1848; Amsteinia Am Stein, 1858.

Trixoclista Townsend, Trans. Amer. Ent. Soc., vol. 19, p. 102, 1892. 1 species. Type, Trixoclista distincta Townsend. Equals Amobia Desvordy, 1830.

Trixodes Coqullletr, Can. Ent., vol. 34, p. 201, 1902. 1 species. Type, Trixodes obesa Coquillett.

$\checkmark$ Trixostomus Rondani, Dipt. Ital. Prodr., vol. 1, p. 130, 1856. 1 species. Type, Ephydra stagnalis Fallen. Equals Scatella Desvoidy, 1830.

$\checkmark$ Trochilodes Coqulletrt, Trans. Amer. Ent. Soc., vol. 29, p. 102, 1903. 1 species. Type, Trochilodes skinneri Coquillett.

Trochobola Osten SAcken, Monogr. Dipt. N. Amer., vol. 4, p. 97, 1869. Change of name for Discobola Osten SACken, 1862, under the mistaken impression that the latter is preoccupied by Discoboli. Type, Limnobia argus SAY. Equals Discobola Osten Sacken, 1862.

Tropidia Meigen, Syst. Beschr. Zweift. Ins., vol. 3, p. 346, 1822. 2 species. Type, Musca scita Harris (as Eristalis milesiformis FALLEN), the second species, by designation of Curtis, Brit. Ent., 1832, p. 401. Syn., Milesiformis Rondani, 1844.

Tropidomyia Williston, Can. Ent., vol. 20, p. 11, 1888. 1 species. Type, Tropidomyia bimaculata Williston.

Tropidopsis Brauer and Bergenstamm, Denkschr. Kais. Akad. Wiss. Wien, vol. 56, p. 132, 1889. 1 species. Type, Tachina pyrrhaspis Wiedemann. 
- Trupanea Schrank, Brief Donaumoor, p. 147, 1795.* 1 species. Type, Mrusca stellata Fuessly (as radiata, new species). Syn., Urellia Desvoiny, 1830; Actinoptera RoNDANI, 1871.

$\checkmark$ Trupanea Macquart, Dipt. Exot., vol. 1, pt. 2, p. 91, 1838. 39 species. Type,

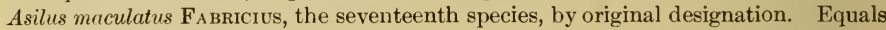
Bactria Meigen, 1820.

$\checkmark$ Trupeta Meigen, Illiger's Mag., vol. 2, p. 277, 1803. 4 species. Type, Musca artemisix Fabricius, the fourth species, by present designation. Equals Euribia Meigen, 1800. (Trypeta of authors equals Terellia Desvoldy).

$\checkmark$ Trypoderma Wiedemann, Auss. Zweifl. Ins., vol. 2, p. 256, 1830. 3 species. Type, Musca americana Fabricius, the first species, by present designation. Equals Cuterebra ClaRK, 1815.

$\checkmark$ Tubifera Meigen, Nouv. Class. Mouches, p. 34, 1800.* No species. Elophilus Meigen, 1803, is a change of name. Type, Musca teuax Linneus. Syn., Elophilus Mrigen, 1803; Eristalis Latreille, 1804; Eristaloides Rondani, 1844; Eristalinus Rondani, 1844; Eristalomya Rondani, 1857; Lathyrophthalmus Miк, 1897.

$\checkmark$ Tylemyia Giglio-Tos, Boll. Mus. Univ. Torino, vol. 8, no. 158, p. 14, 1893. Change of name for Omalocephala Macquart, 1843, not of Spinola, 1839. Type, Omalocephala fusca Macquart. Syn., Omalocephala Macqu 4Rt, 1843, preoceupied.

$\smile$ Tylos Meigen, Nouv. Class. Mouches, p. 31, 1800.* No species. Micropeza Meigen, 1803 , is a change of name. Type, Musca corrigiolata Linneus. Syn., Micropeza Meigen, 1803; Phantasma Desvoidy, 1830.

$\checkmark$ Tyreomma Brauer and Bergexstamu, Denkschr. Kais. Akad. Wiss. Wien, vol. 58, p. 381, 1891. No species. In Biol. Centr.-Amer., Dipt., vol. 2, 1896, p. 293, WulP referred 1 species to it. Type, Tyreomma muscimum WulP.

$\checkmark$ Tyrophaga Kirby, An Intr. Ent., vol.2, p. 283, 1817. 1 species. Type, Musca casei Linneus. Equals Piophila Fallen, 1810.

Tyzenhausia GoRsk I, An. Ent. Imp. Ross., vol. 1, p. 170, 1852.* 1 species. Type, Chrysotoxum vittatum Wiedemann (as respiformis, new species). Equals Sphecomyia Latreille, 1829.

Ubristes W ALK ER, Ins. Saund., Dipt., p. 217, 1852. 1 species. Type, Ubristes flavitibia Walker. Equals Microdon Meigen, 1803.

$\checkmark \quad$ Ugimyia Rondani, Bull. Soc. Ent. Ital., vol. 2, p. 137, 1870. 1 species. Type, Ugimyia sericariz Rondani. Equals Sturmia Desvoldy, 1830.

$\checkmark$ Ula Haliday, Ent. Mag., vol. 1, p. 153, 1833. 1 species. Type, Limnobia macroptera Macquart (as mollissima, new species). Syn., Macroptera Lioy, 1864.

$\checkmark \quad$ Ulidia MeIgen, Syst. Beschr. Zweifl. Ins., vol. 5, p. 385, 1826. Change of name for Chrysomyza Fallen, 1817, on the ground that the latter name is not applicable to all the species. Type, Musca demandata Fabricius. Equals Physiphora Falden, 1810.

$\checkmark$ Ulomorpha Osten SAcken, Monogr. Dipt. N. Amer., vol. 4, p. 232, 1869.1 species. Type, Limnophila pilosella Osten Sacken.

$\checkmark \quad$ Inomyia Meigen, Syst. Beschr. Zweifl. Ins., vol. 1, p. 135, 1818, 4 species (as 5).

Type, Tipula tripunctata FABRIClus, the second species, by present designation. Equals Amphinome Meigen, 1800.

$\checkmark \quad$ Uramya Desvoidy, Essai Myod., p. 215, 1830. 1 species. Type, Uramya producta Desvoldy.

$\checkmark \quad$ Uranotænia Arribalzaga, Rev. Mus. La Plata, vol. 1, p. 405, 1891. 2 species Type, Uranotienia pulcherrima Arribalzaga, the second species, by designation of Neveu-Lemaire, Mem. Soc. Zool. France, vol. 15, 1902, p. 227.

$\checkmark$ Urellia Desvoidy, Essai Myod., p. 774, 1830. 1 species (as 2). Type, Musca stellata Fuessly. Equals Trupanea Schrank, 1795. 
Urophora Desvordx, Essai Myod., p. 769, 1830. 8 species. Type, Musca cardui LinNers (as sonchi, new species), the sixth species, by designation of Westwood, Intr., vol. 2, Synops., 1840, p. 149 . Equals Tephritis Latreille, 1805.

Tafrellia Dessoidy, Hist. Nat. Dipt., vol. 1, p. 674, 1863.1 species. Type, Ceranthia fulvipes Desvordy (as podacina Desvoidy). Equals Ceranthia Desvoidy, 1830.

Vanderwulpia Townsend, Trans. Amer. Ent. Soc., vol. 18, p. 381, 1891. 1 species. Type, Tanderwulpia atrophopodoides Townsend. Syn., Wulpia Brauer and Bergenstann, 1893; Brauerimiyia Townsend, 1908; Catemophrys Townsend, 1908.

Vappo Latreille, Hist. Nat. Crust. et Ins., vol. 14, p. 343, 1805. 1 species. Type, Nemotelus ater Panzer. Equals Pachygaster Meigen, 1803.

Tarichrta Speiser, Berliner Ent. Zeitschr., vol. 48, p. 69, $1903 . \quad$ Change of name for Erigone Desvoidy, 1830, not of SAVIGNy, 1827. Type, MFusca radicum FABricius. Equals Ernestia Desvordy, 1830.

Velocia Coquillett, Can. Ent., vol. 18, p. 158, 1886. 1 species. Type, Anthrax cerberus Fabricius. Equals Hyperalonia Rondani, 1864.

Verrallia Мı,, Wien. Ent. Zeit., vol. 18, p. 137, 1899. 1 species. Type, Cephalops aucta FALLEN.

Verrallina Theobald, Monogĩ. Culic., vol. 3, p. 295, $1903 . \quad 3$ species. Type, Aëdes butleri Theobald, the first species, by present designation.

Villa Lioy, Atti Inst. Veneto, ser. 3, vol. 9, p. 732, 1864.7 species (as 8). Type, Anthrax abbadon Fabricius (as concinnus Meigex), the second species, by present designation. Syn., Hemipenthes Loew, 1869; Storyyx Osten SAcken, 1886; Isopenthes Osten SACken, 1886; Pocilanthrax Osten SACken, 1886; Chrysanthrax Osten Sacken, 1886; Thyridanthrax Osten Sacken, 1886; Hyalanthrax Osten Sacken, 1887.

Viviania Rondani, Dipt. Ital. Prodr., vol. 4, p. 53, 1861. Change of name for Fabricia Meigen, 1838, preoccupied. Type, Tachina cinerea Fallen. Equals Biomya RoNDANi, 1856.

Volucella Geoffror, Hist. Abrégée Ins., vol. 2, p. 540, 1762. 3 species (without names). Type, Musca pellucens Linveus, the first species, by designation of CurTis, Brit. Ent., 1833, 1. 452. Syn., Apivora Meigen, 1800: Pterocera Meigen, 1803; Ornidia St. Fargeau and Serville, 1828; Temnocera St. Fargeau and Serville, 1828; Atemnocera Bigot, 1882; Camerania Giglio-Tos, 1892.

Voria Desvordy, Essai Myod., p. 195, 1830. 1 species. Type, Tachina ruralis Fallen. Syn., Athrycia Desvoidy, 1830; Plagia Meigen, 1838; Blepharigena RonDanr, 1856; Klugia Desvoidy, 1863; Ptilopareia Brauer and Bergenstamm, 1889; Paraplagin Brauer and Bergenstamm, 1891.

Wagneria Desvoiny, Essai Myod., p. 126, 1830. 1 species. Type, Ocyptera costata Fallen (as gagatea, new species). Syn., Scopolia Desvordy, 1830, preoccupied; Brachystylum Macquart, 1855; Phoricheta Rondani, 1861; Ramonda Desvoidy, 1863; Carbonia Desvordy, 1863; Stephania Desvordy, 1863; Ateria, Desvoidy, 1863; Ocalea Desvordy, 1863.

Wahlbergia Zetrerstedt, Dipt. Scand., vol. 1, p. 51, 1842. No species. In 1844, 1 species. Ty'pe, Ocyptera lateralis Fallen. Equals Besseria Desvoidy, 1830.

Walkeria Desvordy, Hist. Nat. Dipt., vol. 1, p. 995, 1863. 1 species (as 50). Type, Tachina simulans Meigen. Equals Exorista Meigen, 1803.

Walshomyia FeLt, 23d Rep. State Ent. N. Y., p. 359, 1908. 1 species. Type, Walshomyia juniperina FeLt.

Willistonia Brauer and Bergenstamm, Denkschr. Kais. Akad. Wiss. Wien, vol. 56, p. 97, 1889. 1 species. Type, Musca esuriens Fabricius. Equals Bèlvoșia Desvoidy, 1830. 
$\checkmark$ Willistoniella Mıк, Wien. Ent. Zeit., vol. 14, p. 136, 1895. Change of name for Rhopalomyia Williston, 1895, not of RübsaAmen, 1892. Type, Ropalomera pleuropunctata Wiedemann. Syn., Rhopalomyia Williston, 1895, preoccupied.

$\checkmark$ Winnertzia Rondan1, Atti Soc. Ital. Sci. Nat. Milano, vol. 2, p. 287, 1860.1 species. Type, Asynapta lugubris Winnertz.

$\checkmark \quad$ Winthemia Desvoldy, Essai Myod., p. 173, 1830. 3 species (as 8). Type, Musca quadripustulata Fabricius (as species 2 to 4 and 6 to 8 ), by designation of Desvoidy, Hist. Nat. Dipt., vol. 1, 1863, p. 207. Syn., Dorbina Desvordy, 1847; Masipoda Brauer and Bergenstama, 1889; Catagonia Brauer and Bergenstamm, 1891.

$\checkmark$ Wohlfahrtia Brauer and Bergensta um, Denkschr. Kais. A kad. Wiss. Wien, vol. 56, p. 123, 1889.2 species. Type, Sarcophila magnifica Schiner, the first species, by designation of Brauer and Bergenstamm, Verh. Zool.-Bot. Ges. Wien, vol. 43, 1893, p. 501. Fquals Sarcophila Rondanı, 1856.

Wulpia Brauer and Bergenstamm, Denkschr. Kais. Akad. Wiss. Wien, vol. 60, p. 128, 1893. 1 species. Type, Wulpia aperta Brauer and Bergenstamm. Not Wulpia B1Got, 1886. Equals Vanderwulpia Townsend, 1891.

$\checkmark$ Wyeomyia Theoвald, Journ. Trop. Med., vol. 4, p. 235, July 15, 1901 . No species. In his Monogr. Culic., vol. 2, November 23, 1901, p. 268, 6 species. Type, Wyeomyia grayii Theobald, the first species, by designation of Neveu-Lemaire, Mem. Soc. Zool. France, vol. 15, 1902, p. 223.

$\checkmark$ Xanionotum Brues, Amer. Nat., vol. 36, p. 376,1902. 1 species. Type, Tanionotum hystrix BRuEs.

$\checkmark$ Xanthandrus Verrall, Brit. Flies, vol. 8, p. 316, 1901. 5 species. Type, Musca comta Harris, the first species, by present designation.

$\checkmark$ Xanthina Aldrich, Kansas Univ. Sci. Bull., vol. 1, p. 92, 1902. 1 species. Type, Xanthine plumicauda A LDRICH.

$\checkmark$ Xanthochlorus Loew, Neue Beitr., vol. 5, p. 42, 1857. 2 species. Type, Porphyrops ornatus HALIDAY, the first species, by present designation. Syn., Leptopus HaLiday, 1831, preoccupied.

$\checkmark$ Xanthocrona WulP, Biol. Centr.-Amer., Dipt., vol. 2, p. 392, 1899. 1 species. Type, Xanthocrona bipustulata W'ulP.

$\checkmark$ Xanthodexia Wulp, Biol. Centr.-Amer., Dipt., vol. 2, p. 256, 1891. 1 species. Type, Tachina cinerea Wiedemann.

$\checkmark$ Xanthogramma Schiner, Wien. Ent. Monatschr., vol. 4, p. 215, 1860 . No species. In his Fauna Austr., Dipt., vol. 1, 1862, p. 319, 3 species. Type, Musca pedissequua Harris (as Syrphus ornatus Meigen), the second species, by designation of Williston, Synop. N. Amer. Syrph., 1886, p. 91.

v Xanthomelana WulP, Tijdschr. Ent., vol. 35, p. 188, 1892.6 species. Type, Xanthomelana gracilenta Wulp, the fifth species, by present designation. Syn., Xanthomelanodes Townsend, 1893.

$\checkmark \quad$ Tanthomelanodes Townsend, Can, Ent., vol. 25, p. 167, 1893. Change of name for Xanthomelana WuLP, 1892, under the mistaken impression that the latter is preoccupied by Tanthomelon Martens, 1860, or by Tanthomelan: Bonaparte, 1850 (a descriptive term for a color-group in the genus Euplectes). Type, Tanthomelana gracilenta Wulr. Equals Xanthomelana Wulp, 1892.

$\checkmark$ Tanthotricha A LDRich, Trans. Ent. Soc. Lond. for 1896, p. 339, 1896. 3 species. Type, Nanthotricha cupulifera ALDRICH, the first species, by present designation. Equals Thrypticus Gerstecker, 1864.

$\checkmark$ Xenochæta Sxow, Kansas Univ. Quart., vol. 2, p. 166, 1894. 1 species. Type, Tenochrta dichromata SNow.

$\checkmark$ Xenomyza Wiedemann, Zool. Mag., vol. 1, pt. 1, p. 60, 1817. 2 species. Type, Damalis planiceps Fabricius, the first species, by present designation. Syn., Chalcidimorpha Westwoon, 1835. 
[Niphandrium Loww, Neue Beitr., vol. 5, p.36,1857. 9 species. Type, Rhaphium quadrifilum Loew, the first species, by present designation. Equals Rhaphium Meigen, 1803. Not American.]

Tiphidicera Macquart, Hist. Nat., Dipt., vol. 1, p. 356, 1834. 1 species. Type, Tiphidicera mifipes Macquart. Equals CEdalea Meigen, 1820.

Tiphura Brulle, Ann. Soc. Ent. France for 1832, p. 206, 1832 . 1 species (as 2). Type, Tipula atrata Linneus. Equals Flabellifera Meigen, 1800.

Tylocrypta KiefFer, Bull. Soc. Ent. France for 1899, p. 69, 1899. 1 species. Type, Ceratopogon fasciata Meigen. Equals Sphæromias Curtis, 1829.

Xylomya Rondani, Dipt. Ital. Prodr., vol. 4, p. 11, 1861. Change of name for Subula Miegen, 1820, not of Schumacher, 1817. Type, Xylophagus maculatus Meigen. Equals Solva W ALKER, 1860.

Yylonhaqus Meigen, Illiger's Mag., vol. 2, p. 266, 1803. 1 species. Type, Nemotelus cinctus De Geer. Equals Erinna Meigex, 1800.

Yylota Meigen, Syst. Beschr. Zweifl. Ins., vol. 3, p. 211, 1822. 16 species. Type, MUsea segnis LiNvees, the ninth species, by designation of CurTis, Brit. Ent., 1832, p. 409. Equals Zelima Meigen, 1800.

Tylotachina Brauer and Bergenstamu, Denkschr. Kais. Akad. Wiss. Wien, vol. 58, p. $342,1891.1$ species. Type, Nylotachina ligniperdæ Brauer and Bergenstamm. Equals Sturmia Desvordy, 1830.

Xylotxja Rondani, Nuovi Ann. Sci. Nat. (Bologna) for 1844, p. 457, 1844. 1 species. Type, Syrphus varus Panzer. Equals Myolepta Newman, 1838.

Yetodesia Rondani, Dipt. Ital. Prodr., vol. 4, p. 9, 1861. Change of name for Aricia Desvoidy, 1830, not of SAvigry, 1817. Type, IIusca impuncta Fallen. Equals Mydæa Desvoidy, 1830. (Yetorlesia of authors equals Phaonia Desvordy.)

Youngomyia FeLt, 23d Rep. State Ent. N. Y., p. 398, 1908. 2 species. Type, Dicrodiplosis podophyllix FeLT, the first species, by original designation.

Yposatcea Rondani, Dipt. Ital. Prodr., vol. 1, p. 198, 1856. No species. Genus formed of Lestremia Macquart, Zygoneura Meigen, and Mimosciara Rondani. Type, Mimosciara molobrina Rondani (the type of Mimosciara), by present designation. Equals Lestremia Macquart, 1826.

Zabrachia Coquillett, Bull. 47, N. Y. State Mus., p. 585, 1901. 1 species. Type, Zabrachia polita Coquillet T.

Zacompsia Coquillett, Ent. News., vol. 12, p. 15, 1901. 1 species. Type, Zacompsia fulva Coquillett.

Zagonia Coquillett, Invert. Pacif., vol. 1, p. 27, 1904. 1 species. Type, Zagonia fara Coquillett. Syn., Siligo Aldrich, 1908.

Zaphne Desvoipy, Essai Myod., p. 527, 1830. 1 species (as 2). Type, Anthomyia divisa MeIGEN.

Zelia Desvoidy, Essai Myod., p. 314, 1830. 5 species. Type, Dexia vertebrata SAY (as rostrata, new species), the first species, by present designation.

Zelima Meigen, Nouv. Class. Mouches, p. 34, 1800.* No species. Eumeros Meigen, 1803, is a change of name. Type, Musca segnis Linnæus. Syn., Eumeros Meigen, 1803; Heliophilus Meigen, 1803; Xylota Meigen, 1822; Micraptoma Westwood, 1840.

Zelinda Desvoldy, Hist. Nat. Dipt., vol. 1, p. 485, 1863. 1 species. Type, Musca libatrix Panzer (as aurulenta, new species). Equals Zenilla Desvoidy, 1830.

Zelleria Desvoidy, Hist. Nat. Dipt., vol. 1, p. 985, 1863. 1 species (as 19). Type, Tachina simulans Meigen. Equals Exorista MEıgen, 1803.

Zelmira Meigen, Nouv. Class. Mouches, p. 16, 1800.* No species. Platyura Me1Gen, 1803, is a change of name. Type, Platyura fasciata Meigen. Syn., Platyura MEIGEN, 1803.

Zenillia Desvoiny, Essai Myod., p. 152, 1830. 2 species (as 3). Type, Musca libatrix PAnzer, the second species, by designation of Desvoldy, Hist. Nat. Dipt., vol. 1, 1863, p. 471. Syn., Nilea Desvoidy, 1863; Atilia Desvoidy, 1863; Clemelis Desvoidy, 
1863; Zelinda Desvo!dy, 1863; Sagaris Desvoldy, 1863; Elpe Desvoidy, 1863; Tritochrta Brauer and Bergenstami, 1889; Myxexorista Brauer and Bergenstamu, 1891.

$\checkmark$ Zetterstedtia Rondani, Nuovi Ann. Sci. Nat. (Bologna) for 1844 , p. 452, 1844. 1 species. Type, Syrphus cimbiciformis Fallen. Equals Mallota Meigen, 1822.

$\checkmark$ Zetterstedtia Desvoidy, Hist. Nat. Dipt., vol. 1, p. 1024, 1863. 1 species (as 14). Type, Tachina simulans Meigex. Equals Exorista Meigen, 1803.

$\checkmark$ Zodion Latreille, Precis, p. 162, 1796. No species. In his Hist. Nat. Crust. et Ins., vol. 3, 1802, p. 444, 1 species. Type, Myopa cinerea Fabricius.

$\checkmark$ Zonosema Loew, Monogr. Trypet., p. 39, 1862.* 1 species. Type, Trypeta meigeni LOEW.

$\checkmark$ Zygobothria Mrk, Wien. Ent. Zeit., vol. 10, p. 193, 1891. 1 species. Type, Sturmia atropivora Desvoldy. Equals Sturmia Desvordy, 1830.

$\checkmark$ Zygomyia Winnertz, Verh. Zool.-Bot. Ges. Wien, vol. 13, p. 901, 1863.6 species. Type, Mycetophila vara StEgER, the second species, by designation of Johannsen, Gen. Ins., Dipt., Mycet., 1909, p. 112.

$\checkmark$ Zygoneura MeIgex, Syst. Beschr. Zweifl. Ins., vol. 6, p. 304, 1830. 1 species. Type, Zygoneura sciarina Mleigen. 


\section{N D E X .}

In this alphabetical list of the specifie names which occur in the previous part of this paper the following style in regard to the generic names has been adopted: Where there are two generic names in the parentheses, the first is the genus in which the species was originally described and the second is the genus of which it is the type; the second generic is in italics if the genus is a synonym and in black face if it is a valid one. Where there is only one generic name within the parentheses it signifies that the species was originally described in, and is the type of, that genus; if the name is in italies it is a synomyn; if in black face the genus is valid.

abbadon Fabricius $(A$

abdominalis Desvoidy (

(Peleteria).......... 586

Say (Dasypogon, Discocephala).. 534

(Dasypogon, Holcocephala). $\quad 552$

Stenhammer (Philygria, Lyto-

gaster)...................

aberrans Rondani (Exorista, Catagonia)...... absobrinus Felt (Culex, Culiseta)........... acerifolius Felt (Oligotrophus, Sackenomyia). acerina Felt (Micodiplosis, Lobodiplosis)..... acrirostris Townsend (Ginglymyia).......... acrostacta Wiedemann (Limnobia, Oligomera) aculeipes Zetterstedt (Anthomyza, Rhyncho-

trichops).

acuta Fabricius ( acuticornis Meigen (Tachina, Acemya)...... (Tachina, Agculocera).... acutirostre Loew (Microstylum, Megapol-

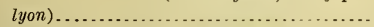
adonis Osten Sacken (Astrophanes)......... adusta Loew (Blepharipeza, Rileya)........... (Blepharipeza, Rileymyia)....... Meigen (Diastata, Trichoptera)........ adustiventris Costa. See albibarbis Meigen.. æacides Loew. See æacus Wiedemann...... æacus Wiedemann (Dasypogon, Ospriocerus). ægeriiformis Gray (Ploas, Lepidophora) ..... ælandicus Linnæus (A silus, Dioctria) ....... ælops Walker ( Tachina, Beskia)............ ( Tachina, Ocypterosipha)...... æmula Meigen ( Tachina, Ethilla)............ æinulans Wulp (Prospherysa) .............. æmulus Meigen (A silus, Stiınogaster) ...... ænea Fabricius (Biblio, Callicera) ............

Fallen (Notiphila, Pelina)............ (Notiphila, Telmatobia) .........

Meigen ( Tachina, Harrisia) ............ ( Tachina, Lydina)............. ( Tachina, Polidea).............. (Tachina, Somoleja)............

Wiedemann. See atra Desvoidy...... ( Tachina, Ennyomma).... (Ortalis, Chætopsis)....... ænescens Desvoidy ( Morellia, Camilla)....... æneus Fallen (Cephalops)................. Scopoli (Conops, Lathyrophthalmus).. ærea Loew ( Myolepta, Chalcomyia)
Page.

æstiva Meigen ( Trypeta, Actinoptera)........ 503 astuans Wiedemann (Culex, Hetcronycha)... 551 æstuum Meigen (Actora) ................. 503 affinis Fallen ( $M$ usca, Agria).............. 504 ( Tachina, Hubneria)......... 553 agarici Olivier ( Mycetophila)............... 572 (Mycetophila, Fungivora)...... 545

agilis Desvoidy, See aterrima Villiers...... 524 See aurifrons Meigen....... 557 See hortorum Fallen....... 571 agrestis Desvoidy. See lineata Fallen...... 562 Fallen. See ungulans Pandelle..... 564 alba Loew ( Trypeta, Aspilota)............. 511

(Trypeta, Neaspilota)........... 574 albescens Zetterstedt. See cinerea Desvoidy. $\quad 549$ albibarbis Meigen (Laphria,Elæotoma)...... 537 albiceps Meigen (Philonicus) .............. 588 albiciliatus Aldrich (A ptorthus) . . . . . . . . . $\quad 509$ albicincta Wulp (Stenodexia) ............... 608 albicollis Meigen ( Tachina, Neaera) ........ 574 (Tachina, Thapsia)....... 614 albicosta Walker (Diastata?, Ischnomyia) ... 556 albifrons Zetterstedt (A ricia, Limnospila).... 561 albimana Macquart. See lasciva Fabricius.. 547 Meigen (Heteroneura) ............ 557 (Heteroneura, Clusiodes)... 525 albincisa Wiedemann( Tachina,A rgyrophylax) $\quad 570$ albipennis Fabricius. See notata Linnæus.. 603

Fallen (Nerina) .............. 575

Meigen (Cecidomyia, Lasioptera).. $\quad 558$ albipes Fallen ( Cordilura, Parallelomma) .... 584 Strom ( Tipula, Dolichopeza)...... 535 (Tipula, Leptina).......... 559 albisquama Zetterstedt (Tachina, Dexodes).. 532 albistalus Williston (Pelagomyia) .......... 586 albistylum Macquart (Ectecephala)........ 536 albitarsis Meigen (Cecidomyia, Porricondyla).. $\quad 594$ Zetterstedt (Aricia, Chirosia) ...... 522 (Homalocephala)....... 553 (Homalocephala,Psairoptera).............. 595 albocostata Fallen (Sciomyza, Chetocera) .... 522 albofasciata Brauer and Bergenstamm. See aberrans Rondani........... 518

Wiedemann. See caucasicus... 506 albolineatus Meigen (Chironomus, Metrioc-

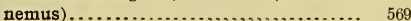


albomaculatus Theobald (Hœmagogus, $\mathrm{Ca}$ comyia) ........................ albomarginatus Curtis. See fasciatus Meigen albomicans Wulp (Didyma).............. alcyon Say (A nthrax, Pocilanthrax)........ aldrichii Stein (Pentacricia) .............. algerica Brauer and Bergenstamm (Rhyncho-

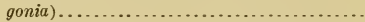
allotalla Meigen (Anthomyia, Aspilia)...... alopex Osten Sacken (Pocota, Crioprora)..... alpestris Jænnicke. See Iongibarbus Loew. alpicola Rondani (Pogonomyia)........... alpigena Mik (Orimargula)............... alpina Meigen ( Trixa, Murana)...........

Zetterstedt (Limnobia, Orimarga).... alternans Zetterstedt (Mycetophila, Brachycampta)........................... alternata Say (Psychoda, Tinearia)......... (Psychoda, Trichoptera)........ amasiæ Brauer and Bergenstamm (Glaucophana)......

ambigua Fallen (Agromyza)

Macquart ( Hystricia, Pseudohystricia ambiguua Macquart (Platypalpus, Crossopalpus).

ambiguus Meigen (Ceratopogon, Forcipomyia) amenides Walker (Clitellaria, Artemita)..... americana Fabricius ( Musca, Trypoderma)...

Johannsen. See underwoodi Underwood

Needham (Oropeza)............

Stein. See siphonina Bigot...... amethystinus Fabricius (Sargus, Chrysochlora)...

ammophilus Loew (Asyndetus) ...........

amœena Macquart (Hystricia)...............

Meigen (Asteia) ..................

analis Jænnicke (Nicocles) . . . . . . . . . . . .

Kirby (Arthria) .....................

Macquart ( Milesia, Cynorhina)......

Meigen (Cordilura, Brachygaster).....

(Sciomyza, Anticheta)........

Schiner ( Trichophora, Siphoniomyia).. ancilla Desvoidy. See simulans Meigen.... angelinæ Meigen ( Tachina, A porotachina) ... angusta Macquart ( Chortophila, Stenogaster). angustata Coquillett (Stenomicra)...

angustatum Aldrich (Leptorhethum)....... angusticornis Townsend (Atrophopalpus).... angustipennis Loew (Ctenophora, Phoroctenia).................

Winnertz (Colpodia)......... anilis Fallen (Dryomyza, Neuroctena) .......

Linnæus ( Musca, Dialineura)......... annosa Zetterstedt (Anthomyza, Quadrula) .. annulata Meigen ( Mycetobia, Plesiastina) ....

(Mycetobia, Symmerus).... annulatus Macquart. See distendens Meigen Schrank ( Culex, Theobaldia).

(Culex, Theobaldinella).

annulipes Macquart (Platystoma, Callopistria)............

(Platystoma, Callopistromyia) ..............

Meigen (Porphyrops, Sympycnus). (Sepsis, Enicita).........

(Sepsis, Enicopus)........

Zetterstedt (Leucopis).

anomala Osten Sacken (Trimicra) .. age

516
Page.

anomala Townsend (Neotractocera). (Siphoplagia)....

Carlier (Anglearia) $\ldots \ldots \ldots \ldots \ldots . . \ldots 506$

Zetterstedt (Callomyza, Agathomyia)..................... 504

anthophila Desvoidy. See radicum Fabricius............................. 539 anthracina Meigen (Psilota)............... 597 anthracinus Loew (Dasypogon, Lastaurus)... 558 anthrax Schrank ( Musca, Anthrax)........ 508 antiqua Meigen ( Tachina, Racodineura)..... 599 (Tachina, Roeselia)......... 601 antisepticus Lichtenstein. See crystallina

De Geer........................... 521 aperta Brauer and Bergenstamm (Oestrophasia, Euostrophasia) 542 (Wulpia).. 620 ( Wulpia, Brauerimyia)... 516 apicalis Brauer and Bergenstamm (Parafrontina $) \ldots \ldots \ldots \ldots \ldots \ldots \ldots \ldots \ldots \ldots \ldots \ldots \ldots$ Coquillett (Mutiloptera) ............ 571 Desvoidy (Peribaea)............... 587 (Sylvia)............... 610

Meigen (Gnoriste)............... 547 apicata Meigen. See apiformis Schrank.... 593 apiformis Schrank (Musca, Dasymyia)...... 531 (Musca, Pocota).......... 593 appendiculata Bigot (Phorostoma, Bathydexia) ................. 513

Loew (Idiotypa)............. 555 (Idiotypa, Neoidiotypa). 575 (Paralimna)......... 584 Macquart (Pangonius, Corizoneura).................. 528 aquila Fallen (Ephydra, Falosoma).......... 544 (Ephydra, Parydra)........... 585 araneæ Coquillett (Pterellipsis) .............. 597 araneoides Dalman (Chionea)............. 522 arctii De Geer. See tussilaginis Fabricius... 518 arcuata Meigen (Phora, Gymnophora)....... 548 arcuatum Mik (Pachystylum, Masistylum)... 565 areuatus Latreille. See subsultan Fabricius). $\quad 571$

Loew (Pelastoneurus, Paraclius)... 583 arenaria Desvoidy. See maritima Haliday.. $\quad 545$ Haliday ( T'achypeza, Chersodromia). 522 argentata Walker (Notiphila, Brachydeutera). $\quad 516$ argentea Fabricius. See tosi Becker........ 570 argenteus Bigot. See limbata Loew........ 566 argentifer Loew. See politus Say........... 598 argentifrons Coquillett (Pseudochaeta)..... 596 Macquart. See prompta Meigen 536 Williston (Desmatoneura)...... 532 argus Say (Limnobia, Discobola) .......... 534

(Limnobia, Trochobola) ........... $\quad 617$ argyragastra Perris (Milichia, Stenoporomyia). 608 argyritarsis Desvoidy (A nopheles, Laverania). $\quad 558$

(A nopheles, Nyssorhynchus)............ 577

argyrophenga Schiner (Lobioptera, Rhynchomilichia).......................... 601 aridella Fallen (Ochtiphila)............... 577 armata Röder (Hystrichodexia) .............. 555 armatus Bigot. See aurata Desvoidy...... 506 Wiedemann (Sargus, Rhaphiocera). 600 arrogans Linnæus (Musca, Sicus).......... $\quad 605$ 
Page.

artemisiæ Fabricius ( Musca, Euribia)....... 542 (Musca, Forellia)....... 545

( Musca, Trypeta).......

Felt (Diathronomyia)

arvensis Desvoidy. See carnaria Linnæus.. arvicola Meigen ( Tachina, Cynisca) .......... arvorum Desvoidy. See simulans Meigen... asellus Wheeler (Parasyntormon)............ asilicus Fallen (Syrphus, Criorhina).........

(Syrphus, Penthesilia)........ asiliformis Latreille. See muscarius Fabri-

cius.................................. assimilis Fallen ( Musca, Blissonia).......... (Tachina, Chetogena)........ ( Tachina, Phorocera)......... ater Coquillett(Thlipsogaster,Parabombylius). Linnæus (A silus, Andrenosoma)........

Panzer (Nemotelus, Pachygaster)

(Nemotelus, Vappo) ............. aterrima Fabricius ( $M$ Musca, Noda).......... ( Musca, Philodendria).... ( Mfusca, Phora)........... (Musca, Trineura).......

Villiers ( Mfusca, Clelia)............ atomaria De Geer (Tipula, Atomaria)........ atra Brauer and Bergenstamm (Gaediophana).

Coquillett (Apinops) .................. See helymus Walker.........

Desvoidy (Clytia, Myiophasia)........... (Clytia, Phasioclista)...........

Fabricius ( Myopa, Occemya)........... ( Myopa, Thecophora).........

Macquart (Penthetria, Eupeitenus).......

Meigen. See aterrima Fabricius....... See fasciata Wiedemann...... (Conicera) .................... (Erioptera, Molophilus) .......... (Gonia, Rhedia) .................

Williston (Ochtheroidea) ............... atrata Linnæus ( Tipula, Ctenophora)........ ( Tipula, Flabellifera)........ (Tipula, Tanyptera)

( Tipula, Xiphura).

atratus Hine (Snowiellus). atriceps Loew (Analcocerus) atricornis Meigen (Heleomyza, Allophyla).... atripennis Coquillett (Chaetoplagia).......... atrophopodoides Townsend (Vanderwulpia). atropivora Desvoidy (Sturmia, Zygobothria).. atrox Williston (Hyperechia, Dasylechia).... aucta Fallen (Cephalops, Verrallia).......... See furcatus Egger ............ aulicus Meigen (Porphyrops, Eutarsus)....... aurantiaca Kieffer (Joannisia).............. aurata Desvoidy (Hemyda).

(Hemyda, A ncylogaster) .... auratus Macquart (Pangonius, Scaptia)....... aurea Fallen ( Tachina, Leskia).............. (Tachina, Pyrrosia) ............. aureorufa Philippi (Eriophora) auribarbis Macquart. Seechalcogaster Wiedemann ................................. aurifacies Desvoidy. See flaviceps Meigen... aurifer Coquillett (Culex, Pseudoculex).......
Wiedemann (Ortalis, Automola)....

Page. aurifex Wiedemann (Microdon, Chymophila). $\quad 524$ auriflamma Wiedemann (Cyphomyia)...... $\quad 530$ aurifrons Meigen ( Tachina, Labigastera) ..... 557 Townsend (Paradexodes)........... 584

auripila Brauer and Bergenstamm (Dexodes,
Allophorocera) ........................ 505 auropubescens Latreille. See mutabilis Linnæus................................ aurulenta Desvoidy. See libatrix Panzer... aurulentus Fabricius (Dasypogon, Ceraturgus) austriaca Meigen (Rhingia) autissiodorensis Desvoidy ( Ifufetia).......... avicularia Linnæus (Hippobosca, Ornithomyia)...

avium Macquart (Strebla) .................. azurea Fallen (Musca, A vihospita)...........

( Musca, Protocalliphora)...... bakeri Coquillett (Chaetoclusia) ............. barbata Bigot (Phorocera, Metadoria) ....... Coquillett (Hypostena, Methypostena) barbatus Fabricius (A silus, Eccritosia)....... Osten Sacken. See nitidulus Fabricius..

Scopoli (Erax) .................. barberi Coquillett (Anopheles, Coelodiazesis).. barbicornis Fabricius. See communis Meigen.................................. barbipes Meigen (Sepsis, Beggiatiía) ......... basalis Loew (Gloma, Oreogeton) ............ basilica Snow (Polymorphomyia)............. beauvoisii Desvoidy (Prionella) ............. beccariina Rondani (Ornithoica)............. bechsteinii Kolenati (A crocholidia)........... bellus Loew (Blax) ....................... (Blax, Blacodes)................ (Blax, Loewiella) ............... (Tanypus, Psilotanypus) - -2.0 .2$

(Phthiridium, Stylidia)............ 610

bicincta Desvoidy. See bifasciata Fabricius. $\quad 513$ Linnæus ( Musca, Antiopa) ......... . 508 (Musca, Chrysotoxum).... 524 bicinctus Fabricius ( Tabanus Diabasis)...... 532 (Tabanus, Diachlorus)... 532

bicolor. See curvipes Wiedemann ......... 593 Fabricius (Syrphus, Paragus) ....... 584 Giglio-Tos (Mesembrinella) ....... 568 Jænnicke. See diaphana Fabricius. $\quad 509$ Loew (Sigalöessa)................. 605 (Synamphotera, Boreodromia).................... 515 Macquart. See curvipes Wiedemann. 593 Meigen (Nematocera)............... 574 (Tachina, Ceromya)......... 520

Wiedemann ( Tabanus, Atylotus)..... 512 (Tachina, Parafabricia)... 584 bifasciata Fabricius ( Musca, Belvosia)....... 513 (Musca, Latreillia)....... 558 (Musca, Latreillimyia).. 558 bifurcata Fallen. See lutea Panzer.......... 534 bifurcatus Linnæus (Culex, Anopheles)...... 507 bigoti Macquart (Pomacera)................ 594 bigotii Bellardi (Culex, Lutzia)............. 563
Westwood (Nothra)............... 576 
Page.

biguttata Meigen. See bisignata Meigen.... 534 bilbergi Zetterstedt (Anthomyza, Prosalpia).. 595

bilimeki Brauer (Cynipimorpha).......... 529 Brauer and Bergenstamm (Prorhynchops) ....................... bilimekii Brauer and Bergenstamm (Estrophasia, Neoptera)...............

Brauer and Bergenstamm (Phasiopteryx $) \ldots \ldots \ldots \ldots \ldots \ldots \ldots \ldots$

bimacula Walker (Euarmostus)............ bimaculata Hartig ( Tachina, Schaumia)..... Loew. See maculipennis Walker Meigen (Mycetophila, Lejomya).. ( Mycetophila, Lejosoma).. Schummel ( Tipula, Dicranota)... Williston (Tropidomyia).......... bipartita Desvoidy. See nigripes Fallen.... bipunctata Fallen (Geomyza, Mycetaulus)... Linnæus ( Tipula, Labidomyia)... Scopoli ( Musca, Chrysochroma).. (Musca, Chrysonotus).... (Musca, Chrysonotomyia).

bipustulata Wulp (Xanthocrona)............ bisangula Rondani (Gonioneura)............ bisignata Meigen ( Tachina, Diplomera)...... (Tachina, Spylosia)........ bisulcatus Coquillett (Micraëdes) ........... biumbrata Wahlberg. See albitarsis Zetterstedt. .

bivittata Coquillett ( Myocera, Dolichocodia).. bivittatus Loew (Bombylius, Triplasius).... blanda Osten Sacken (Exorista, Eusisyropa). blasigii Mik (Parathalassius)................ blasii Kolenati (Listropoda) ................. blattoides Meinert (Aenigmatias) ............ blondeli Desvoidy. See sicula Desvoidy.... bombiformis Fallen (Syrphus, Arctophila)... bomboides Wiedemann (Asilus, Mallophora). bombylans Desvoidy. See gnava Meigen... Fabricius (Milesia, Temnostoma) (Stomoxys, Dejeania)..

borealis Coquillett (Eutanypus)............ Linnæus (Empis, Platypterygia)..... boscii Desvoidy (Parthenia)................. bovina Desvoìdy. See denticulatus Meigen. bovinus Linnæus (Tabanus) ................ bovis Linnæus Oestrus (Hypoderma)........ brakeleyi Coquillett (Corethrella)........... brasiliana Brauer and Bergenstamm (Masiphya).................... Brauer and Bergenstamm (Synthesiomyia) ....................

brasiliensis Schiner (Aphestia)............. (Atacta) ................

Westwood (Linnobiorhynchus)... brassicaria Fabricius ( $M u s c a$, Cylindromyia). (Musca, Ocyptera) .....

braueri Strobl. See galeata Haliday.........

Williston (Atrophopoda, Diaphoro-

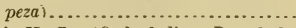
brevicornis Kieffer (Orthocladius, Dactylocladius)................. Macquart. See simulans Meigen. 561 535 brevicornis Say (Diopsis, Sphyracephala)... Wiedemann (Laphria, Tapinocera) ............. (Mydas, Dolichogaster).......... 535

brevifrons Walker (Hesperinus) $\ldots \ldots \ldots \ldots \ldots . \quad 551$ brevinervis Rondani (Siphunculina) .......... 606 brevipennis Curtis. See atra Meigen........ 571 Meigen (Chlorops, Elachiptera)... 537 Zetterstedt. See arenaria Haliday ........................ 522 brevipes Fabricius (Dacus, Senopterina)..... 605 brevirostris Macquart (Bombylius, Sparnopolius)...

Coquilett (Paradmontia) ............. 584 browni Kellogg (Eretomoptera)............ 539 buccalis Desvoidy (Albinia) .............. 504 buccata Desvoidy. See stagnalis Fallen..... 603 Fallen (Heteromyza)............. 551 (Heteromyza, Aedoparea)..... 578 (Heteromyza, Exocheila)...... 544 (Heteromyza, Heterocheila).... 551

(Heteromyza, Heterostoma).... 551

(Musca, Hylephila)........... 554

Linnæus (Conops, Myopa)......... 573 bucephala Meigen ( Tachina, Cnephalia)...... 525 bulbosus Osten Sacken (Scenopinus, Metatrichia)........................... 568 busckii Coquillett (Aspidoptera)........... 511 butleri Theobald (Aёdes, Verrallina)........ 619 byssina Schrank ( Tipula, Camptocladius)... $\quad 518$ cada verina Linnæus ( Musca, Pyrellia)....... 598 cæcutiens Linnæus ( Tabanus, Chrysops).... 524 cæsar Linnæus ( Musca, Lucilia)........... 563 cæsia Macquart (? Chortophila, Psilometopia). $\quad 597$ cæsio Harris (Musca, Euleia) ............. 541 (Musca, Philophylla)......... 588 calcaneus Loew (Callinicus) ............. 517 calceata Fallen (Calobata, Rainieria)........ 599

(Calobata, Tanipoda).......... 612 calcitrans Linnæus (Conops, Stomoxys)...... 609 californica Bigot ( Thevenemyia)............. 614 californiensis Macquart (Micropalpus, Deopalpus)................................ caliginosa Meigen (Phora, Anevrina)......... calogaster Bigot (Microphthalma, Macrome-

topa). ............................. 564 calopus Loew (Lepidomyia)............... $\quad 559$ (Lepidomyia, Lepidostola)..... $\quad 559$ Meigen (Culex, Stegomyia).......... 608 calvicrura Coquillett (Lasiops, Opsolasia).... 580 campestris Desvoidy. See hæmorrhoa Meigen............... 571 (Limosia)........... 561 561
529
577

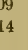

\section{1} 506

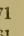

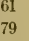

Fallen ( Tachina, Ophelia) ....... 579 Latreille (Pipunculus).......... 591 (Pipunculus, Dorilas)... 535 (Pipunculus, Microcera). $\quad 569$ eanadensis Loew ( Trypeta, Epochra)........ 539 Theobald (Culex, Culicada)...... 529 Westwood. See westwoodi Osten Sacken.................... 537 ge.
607 2
5
1
6
1
7

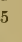

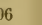

10

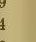
25

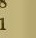

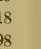

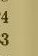

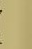

(n)

,

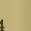

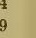

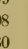


Page.

canalis Coquillett (Lordotus, Geminaria)..... 546 canariensis Bergroth (Geranomyia, A porosa). . 509 cancer Theobald (Deinocerites)............ (Deinocerites, Brachiomyia). (Deinxcerites, Brachiosoma). candida Coquillett ( Efferia).

canescens Meigen (Heleomyza, Tephrochlamys) ..................

Wheeler (Parhydrophorus) ......

canicularis Linnæus ( Musca, Homalomyia).. (Musca, Myantha)...... ( Musca, Philinta)...... canina Fabricius ( Musca, Dexiosoma)...... eapensis Desvoidy. See bombylans Fabricius. . . . . . . . . . . . . . . . . . . . . . capitata De Geer. See ornata Meigen.......

(Musca, Gonia)...........

( Musca, Salmacia).......

Wiedemann ( Trypeta, Ceratitis).... ( Trypeta, Halterophora) ( Trypeta, Petalophora).

capito Loew (Oedopa)

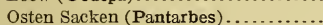
capnopterus Wiedemann (Dasypogon, Archilestes) .............

(Dasypogon, Archilestris)...........

capucina Fabricius (Bibio, Exoprosopa)..... carbonaria Panzer ( Musca, Scopolia)....... cardui Linnæus ( Musca, Urophora)......... Meigen (Anthomyia, Delia).......... caricicola Desvoidy. See palustris Fallen... carnaria Linnæus ( Musca, Myophora)...... (Musca, Phorella)......... ( Musca, Sarcophaga)..... carnea Loew (Lestremia, Cecidogona)....... carnivorus. See diadema Linnæus.......... carolinensis Brauer and Bergenstamm. See

tibialis Desvoidy.

casei Linnæus ( Musca, Piophila) .......... ( Musca, Tyrophaga) ........ caucasicus Fischer (Rhynchocephalus, Andrenomya). caudata Fallen (Notiphila, Dichaeta) . Zetterstedt (Cordilura, Okenia)...... (Cordilura, Okeniella)... caudelli Dyer and Knab ( Mochlostyrax)..... celeripes Meigen ( Tachydromia, Agatachys)... cellaris Meigen. See funebris Fabricius...... cellarum Desvoidy (Bacchis) .............. cerasi Linnæus ( Musca, Rhagoletis)......... cerberus Fabricius (Anthrax, Velocia)........ cerealis Rondani. See destructor Say....... cereris Fallen (Oscinis, Centor)............ (Oscinis, Cetema)............ cervi Linnæus (Pediculis, Alcephagus)...... (Pediculus, Haemobora) ...... (Pediculus, Lipoptena)........ (Pediculus, Ornithobia) ....... cervina Panzer. See cervi Linnæus....... . cervinus Loew (Aphoebantus)............ chætosula Townsend (Muscopteryx)......... chalcogaster Wiedemann (Asilus, Lophono-

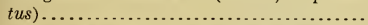
chalconota Meigen ( Tachina, Gymnopsis).... ( Tachina, Javetia)....... ( Tachina, Macquartia).... ( Tachina, Rondanimyia)... chalcoproctus Loew (Sphageus) $\ldots \ldots \ldots \ldots \ldots .607$ chalybea Meigen (Actina)................. 503 chalybeata Forster ( Musca, Beris)......... 513 (Musca, Hexacantha) .... 552 chamæleon Linnæus ( Musca, Hoplomyia)... 553 ( Musca, Stratiomys).... 609 cheloniæ Rondani (Exorista, Paraëxorista)... 584 chichimeca Wheeler and Melander (Empis,

Lamprempis). chirothecata Scopoli (Tipula, Penthoptera)... 586 chittendeni Coquillett (Paraphyto).......... 585 chorea Fabricius ( $M u s c a$, Lonchaea)......... 562 chrysanthemi Loew (Clinorhyncha)....... 525 chrysitis Meigen (Asilus, Machimus)....... 564 chrysocoma Osten Sacken (Pangonius, Goniops)......................... 547 chrysoprocta Wiedemann (Tachina, Macromeigenia)......................... 564 ciliata Fabricius ( Musca, Microcera)........ 569 Macquart. See scutellala Desvoidy .. 514 ciliatus Fabricius (Culex, Psorophora) . ...... 597 cilicrus Kieffer (Diplosis, Clinodiplosis) . .... 525 ciligera Desvoidy. See devia Fallen........ 580 See dubia Fallen........ 538 See inanis Fallen........ 523 cilipennis Macquart. See melania Meigen... 617 cimbiciformis Fallen (Syrphus, Zetterstedtia). 622 Loew. See stigma Fabricius.. $\quad \mathbf{5 7 6}$ cimicoides Fabricius. See arrogans Linnæus. 605 See connexa Meigen. . 611

cincta Fabricius ( Tipula, Pelopia) .......... 586 (Tipula, Protenthes)........ 595

(Tipula,Tanypus).......... 612

Giglio-Tos (Myioscotiptera) .......... 572

Kieffer (Holoneura) ................ 552 cinctus De Geer (Nemotelus, Erinna)...... 539 (Nemotelus, Pachystomus) .. 582 (Nemotelus, Xylophagus)... 621

Osten Sacken (Aochletus) .......... 508 cineraria Rondani (Cestonia) .............. 520 cinerascens Meigen (Limnobia, Marginomyia). $\quad 565$ Rondani (Gonia, Pseudogonia)... 596 cinerea Coquillett (Ateloglossa)............ 511 (Parodinia).............. 585

Desvoidy (Leucophora) ............. 560 (Leucophora, Hammomyia) 549 (Leucophora, Ocromyia)... 577

Fabricius ( Myopa, Zodion)........... 622

Fallen (Notiphila) . . . . . . . . . . . . 576

(Notiphila, Keratocera)......... 557

( Tachina, Biomya)........... 514

(Tachina, Fabricia)............ 544

(Tachina, Viviania).......... 619

Loew (Rhicnoessa).............. 600

Marquart (A peilesis).............. 508

(Lestremia)............. 560

Townsend (Trepophrys) ............ 615

Wiedemann ( Tachina, Xanthodexia). 620

Wulp (Microchaetina)............. 569 cinereus Latreille. Seegeniculata De Geer... 516

Wiedemann (Aëdes) ............. 504 cingulata Desvoidy $($ Actia $) \ldots \ldots \ldots \ldots \ldots \ldots . \quad 503$ (Actia, Elfia $\ldots \ldots \ldots \ldots . \quad 537$ $\begin{array}{llll}562 & (\text { Actia }, \text { Elja }) \ldots \ldots \ldots . . . & 537 \\ 548 & \text { cingulatus Fabricius (A silus, Epitriptus)..... } & 539\end{array}$ citriperda MacLeay. See capitata Wiedemann.............................. 519 civilis Rondani ( Tachina, Ptilotachina)...... 598 
elaripennis Desvoidy. See albocostata Fallen.............. 522

(Kockia) ............ 557 (Scatina)............ 603 Melander (Prorates) ........... 595 clausa Bigot (Hyadesimyia)............... 553 Brauer and Bergenstamm (Estrophasia)....................... Macquart. See obsoleta Meigen..... Osten Sacken (Hirmoneura Parasymmictus $)$........................ elavata Drury ( Musca, Mydas)............. claviger Osten Sacken (Phyllolabis).......... elavipes Fabricius (Dictya, Ropalomera)......

(Laphria, Lampria)....... (Syrphus, Lampetia)......

- (Syrphus, Merodon).......

( Tipula, Bittacomorpha)..

clelia Osten Sacken (Stonyx).

clistoides Townsend. See ænea Wiedemann. elunipes Fallen ( Milesia, Sphegina).......... coaretata Fallen? (Ephydra, Hygrophila)..... (Ephydra, Napæa) ..........

( Musca, Gastrolepto).......

Perty (Laphria, Planetolestes)...... coaretatus Loew (Microdon, Omegasyrphus). cocciphila Coquillett (Phora, Syneura)....... cœeruleonigra Maequart (Jurinia, Jurinella). cœerulescens Loew (Lyroneurus)............. Macquart (Senogaster).......... cognata Meigen (Musca, Melinda) ............ Wiedemann (Tephritis, Acidia)...... (Tephritis, Epidesmia). cognatus Wiedemann (Tephritis, Prionimera) coleoptrata Fabricius. See hemipterus Fabricius.

Scopoli ( Musca, Stegana)........

collaris Fallen ( Tachina, Degeeria)............ ( Tachina, Medina)........... collusor Melander (Hemerodromia, Metachela) colombaschensis Fabricius (Rhagio, Simulium)

comantis Coquillett (Empimorpha).......... comaster Williston (Apatolestes)............. combinata Linnæus ( Musca, Balioptera).... (Musca, Geomyza).... communis Desvoidy. See flaveola Fabricius Meigen (Ceratopogon).

(Ceratopogon, Helea) ..... complexa Osten Sacken (Teucholabis)....... complexus Bigot (Ptychoproctus)........... comstockii Townsend (Eulasiona)........... comta Fallen ( Tachina, Bonnetia)........... (Tachina, Marshamia)......... (Tachina, Micropalpis)........

Harris (Musca, Xanthandrus)........ coneavus Aldrich (Cœloglutus) .............. concinnata Meigen ( Tachina, Compsilura)... ( Tachina, Doria).......... (Tachina, Machæræa).... concinnus Meigen. See abbadon Fabricius . confinis Fallen ( Tachina, A plomya).......... confirmatus Arribalzaga (Ochlerotatus) ..... confusus Theobald (Sabethoides)...........

572

590

601

557

557

567

514 609

538

607

554

573

546

591

579

610

556

563

605

567

502

538

594

587

608

531

565

568

605

537

508

513

546

536

520

549 613

598

541

515

565

569

620

526

526

535

563

509

602
Page.

conica Desvoidy (A mobia).

Townsend (Ceratomyiella) ........... 520

Wiedemann (Philopota)............. 588

coniophaga Winnertz (Diplosis, Mycodiplosis) 572 conjungens Walker. See fermosa Harris .... 539 connexa Meigen ( Tachydromia)............ 611

( Tachydromia, Coryneta).... 528

(Tachydromia, Tachista)..... 611

619

577

innæus ( Musca, Ceria) ........

Macquart (Mixogaster)..........
contaminata Linnæus ( Tipula, Liriope)......

( Tipula, Ptychoptera)

contigue Walker. See lasiophthalmus Mac-

quart............................ 524

continua Panzer ( Musca, Clytia)........... 525

(Musca, Clytiomya)....... 525

contristans Wiedemann (Dolichopus, Lepto-

pus)............................. 560

convecta Walker ( Tachina, Schizotachina)... 604

coprina Desvoidy (Lordatia) ............... 563

cordyluroides Stein (Phyllogaster)......... 590

cornicina Fabricius ( Musca, Euphoria)...... 542

(Musca, Orthellia)...... 581

(Musca, Pseudopyrellia). 596

cornuta Brauer and Bergenstamm. See

aelops Walker................. 573

Fabricius (Musca, Cerajocera) ...... 519

Fallen (Oscinis, Crassiseta)........ 528

(Oscinis, Macrochetum)...... 564

Meigen (Sepsis, Acrometopia)........ 503

cornutus Wiedemann (Dasypogon, Ceratur-

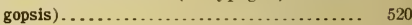

corrigiolata Linnæus ( Musca, Micropeza).... 569

(Musca, Phantasma)... 587

(Musca, Tylos)........ 618

coryleti Scopoli ( Mfusca, Pherbina)......... 588

(Musca, Tetanocera)........ 613

costalis Gerstaecker (Diacrita) ............ 532

(Diacrita, Carlottæmyia).. 518

von Roser (Phora, Megaselia)...... 566

costata Fallen (Ocyptera, Carbonia)......... 518

(Ocyptera, Ocalea)............ 577

(Ocyptera, Phoricheta)....... 589

(Ocyptera, Wagneria)........... 619

Wulp (Acaulona) ............... 502

cothurnata Wiedemann (Stomoxys, Stomato-

dexia) ............................. 609

erabroniformis Linnæus (Asilus) ........... 511

crassicornis Meigen (Tachina, Gymnopareia). 548

(Tachina, Gymnophtalma) 548

crassineura Rondani. See costalis von Roser. 566

crassipennis Fabricius ( Musca, Melieria).... 567

crassipes Fabricius (Hæmatopota, Hadrus)... 549

(Hxmatopota, Lepiselaga). $\quad 559$

Loew (Hormomyia)............... 553

Panzer (Chironomus, Eurycnemus). 543

crassirostris Meigen. See flavipennis Fallen. 582

crawfordii Coquillett (Pseudacteon) ......... 595

crawii. See diabroticae Shimer............ 519

Coquillett. See diabroticae Shimer. 578

crebra Wulp (Prospherysa, Phrissopolia)..... 589 crepusculi Brauer and Bergenstamm (Cne-

phaotachina) ......................... 
Page.

crinicornis Wiedemann (Psilopus, Megisto-

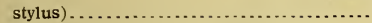
crocata Linnæus ( Tipula, Pachyrhina)....... cruciger Wiedemann. See acuta Fabricius.. crudelis Wiedemann ( Tachina, Angiorhina).. crystallina De Geer ( Tipula, Chaoborus)..... cucularia Linnæus ( Irusca, Gymnostyla)..... cucullaria Linnæus ( Musca, Elgiva)......... culiciformis De Geer ( Tipula, Corethra)..... culta Wiedemann (Trypeta, Paracantha).... cuniculi Clark (Oestrus, Cuterebra).......... cuprarius Linnæus ( $\mathrm{Musca}$, Geosargus).....

(Musca, Sargus)......... cupreiventris Wulp (Telothyria)............. cupreus Fallen (Dolichopus, Gymnopternus). Scopoli (Conops, Chrysoclamis)...... (Conops, Ferdinandea)...... cupulifera Aldrich (Xanthotricha)............ cursitans Fabricius ( Musca, Platypalpus).... curvicauda Gerstaecker (Toxotrypana)....... (Toxotrypana, Mikimyia)..............

curvipes Fabricius. See clavipes Fabricius. .

$$
\text { (Damalis). }
$$

Fallen (Dolichopus, Campsicnemus). Latreille. See subsultans Fabricius. Wiedemann (Merodon, Polydonta)..

(Merodon, Polydontomyia ...............

(Merodon, Triodonta)..

curvirostris Laveran (Simondella) ........... eyanea Giglio-Tos (Bricenniella)............ cyaneiventris Macquart (Blepharipeza, Paragaedia)

cyanescens Coquillett ( Culex, Lepidosia)..... cyaneus Fabricius ( Culex, Sabethes)......... cyanocephalus Meigen (Diaphorus, Brachypus).................................. cyanurus Loew (Asilus, Itamus)............ (Asilus, Neoitamus)........ cylindrica Desvoidy. See collaris Fallen ....

See melanocephala

Meigen.............

Fabricius ( $M u s c a$, Nemopoda).... cylindricornis Fabricius ( Musca, Lauxania).. cylindricus De Geer (Asilus, Gonypes)........ (Asilus, Leptogaster).... cynipsea Linnæus ( Musca, Sepsis).......... . dahlii Meigen (Clitellaria, Adoxomyia)........ dakotensis Townsend (Euscopolia)..........

(Sarcoclista)..........

debilis Loew (Elliponeura)................ decemnodia Scopoli. See notata Linnæus... decens Townsend (Eumacronychia)......... . decipiens Meigen. See pedella Fallen....... decisa Walker ( Tachina, Pararchytas)........ decora Loew (Pteroptila)...................

Macquart (Somula) ................. decorata Loew (Diplosis, Bremia) .......... defecta Williston (Manota)................ demandata Fabricius ( Musca, Chloria)...... ( Musca, Chrysomyza).... ( Musca, Physiphora)..... ( Musca, Ulidia) ........... demoticoides Brauer and Bergenstamm (Arthrochaeta).

denticornis Panzer (Chlorops, Ceratomyza)... $\quad 520$

566

582

591

506

521

548

537

528

583

529

546

603

612

548

523

545

620

592

615 (Chlorops, Cerodontha)... 520 (Chlorops, Odontocera).... 578 denticulatus Meigen (Borborus, Coprina).... 527 depila Meigen. See flavipes Panzer......... 521 depressariæ Brauer and Bergenstamm (Catachxta).............................. 518 depressus Macquart (Pangonius, Diatomineura)............. 532 (Pangonius, Osca) ...... 581 destructor Say (Cecidomyia, Mayetia)....... 565 (Cecidomyia, Phytophaga).... $\quad 590$ devia Fallen ( Tachina, Brachicoma)......... $\quad 575$ ( Tachina, Oppia)............. 580

Haliday (Orphnephila).............. 581 (Orphnephila, Chenesia)....... 522 (Orphnephila, Thaumalea)...... 614 diabroticæ Shimer (Melanophora, Celatoria). $\quad 519$ diadema Fabricius (Asilus, Cheilopogon)..... 521 (A silus, Dasypogon)...... 531 (Asilus, Seilopogon)...... 605 See melancholia Harris... 511

Linnæus (Musca, Chrysopilus)..... 523 (Musca, Medetera)....... 565

Meigen (Chlorops, Haplegis)....... 549 diademoides Townsend. See triquetra Olivier.............................. 585 diaphana Fabricius (Musca, Archilestris)... 509 (Musca, A rgyra)........ 509

(Musca, Lasiargyra)..... 557

(Musca, Porphyrops).... 594

dichromata Snow (Xenochaeta)............ 620 didyma Loew (X ysta, Clistomorpha)....... 525 dimidiata Loew. See argentata Walker..... 516 Macquart. See leucoprocta Wiedemann........................ 578

Staeger ( Mycetophila, Mycothera).. 572 diophthalma Linnæus ( Musca, Milesia)..... 570 diophthalmus Fabricius. See saltuum Fabricius ............................ 607 directa Walker (Pangonius, Scarphia)....... 603 discolor Coquillett (Culex, Ceratocystia)...... 519 discrucians Walker (Culex, Janthinosoma)... 556 disjuncta Wiedemann (Anthrax, Lepidanthrax)............. 559 (Tachina, A mesia).... 505 ( Tachina, Microphthalma) .............. 570

dispar Desvoidy. See quadrum Fabricius... 572 Rondani. See occulta Meigen....... 547 disparata Brauer and Bergenstamm (Plagiomima) ........................... 591 dispellans Walker (Tipula, Brachypremna). $\quad 516$ dissecta Meigen (Anthomyia, Gymnogaster)... 548 dissimilis Meigen (Sarcophaga, Hartigia).... 549

Walker. See elongata Say....... 532

Wulp (Nemochæta).............. 574

distendens Meigen (Chrysotus, Nematoproctus) $\ldots \ldots \ldots \ldots \ldots \ldots \ldots \ldots \ldots . \quad 574$ Wiedemann (Asilus, Ampyx) ...... 506 (A silus, Doryclus) ... 535 distincta Meigen ( Tachina, Hypochæta)..... 555 Townsend ( Trixoclista).......... 617 Winnertz. See sylvatica Curtis.... 613 distinctissima Meigen (Limnobia, Cylindro-

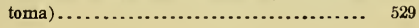


dives Wiedemann. See litturata Olivier ... 540 divisa Meigen (Anthomyia, Zaphne)......... 62

Loew. See immaculata Macquart... dolium Fabricius (Ocyptera, Lissa)......... (Ocyptera, Megamerina).. dolosa Arribalzaga. See æstuans Wiedemann domestica Linnæus (Musca) dominula Desvoidy. See simulans Meigen. dorsalis Fabricius ( $\mathrm{Fyopa,} \mathrm{Lonchopalpus)...}$ (Stomoxys, Arpagita) .....

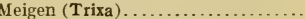

dubia Bigot (Rhamphinina)............... Fallen ( Tachina, A poromya).........

( Tachina, Enthesis)...........

( Tachina, Lyphe).............

Kieffer (Bryocrypta) ..............

Macquart (Conosia, Eriostyla)........ (Oscinis, Botanobia) .......

dufourii Westwood. See westwoodi Guerin-

Meneville

durhamii Theobald (Limatus) ............. dux Eschscholz ( Musca, Compsomyia)...... dyari Coquillett ( Culex, Culicella).......... echinura Desvoidy (Salia)................ (Salia, Spoggosia)....... edwardsii Loew ( $A$ llocotus)

(Allocotus, Paracosmus)....

effrons Giglio-Tos (Brachiophyra).......... egregia Wulp (Polygaster).............. ehrmanni Coquillett (Aldrichia).......... elegans Coquillett (Amphicosmus).........

(Johnsonia).

Desvoidy. See hortulana Rossi.... Meigen (Callomyia)................. (Callomyia, Cleona)........... (Chrysogaster, Orthonevra)... (Phytomyza, Dinevra)....... (Phytomyza, Napomyza)...

Panzer (Chamaemyia)............. Wiedemann (Cyphomyia, Euparyphus).

elegantula Röder (Agathon) ................ elegantulus Wiedemann (Dasypogon, Stichopogon)

elongata Say (Stygia, Agnotomyia)......... (Stygia, Dialysis)

Wiedemann (Clitellaria, Acanthina) (Clitellaria, Acanthinomyia)........... $50^{\circ}$

Wulp (Paneryma) ............... $5 \div 3$ elongatus Fabricius (Syrphus, Baccha)..... 513 enigma Coquillett (Ptilomyia) ........... $598^{\circ}$ ephippiata Fallen ( Tachydromia, Elaphropeza).

ephippium Fabricius ( Mrusco, Neria) ........ Stratiomys, Clitellaria) episcopus Osten Sacken (Rhaphiomidas) ... eques Wiedemann (Dexia, Pseudodexia).... . equi Clark. See intestinalis De Geer....... equina Fallen (Copromyza) ...............

(Copromyza, Mycetia)........

Linnæus (Hippobosca)...

(Hippobosca, Nirmomyia)

erigonea Desvoidy ( Mericia)............... erinacea Fabricius ( $\mathbf{F u s c a}$, Peteina)......... erosa Loew (Epiplatea)
Page.

errans Meigen (Agromyza, Phytobia)........ 590

(Anthomyia, Trennia)........ 615

erratica Fallen ( Musca, Phaonia)........... 587

erythrocephala Fabricius. See morio Fabricius .................. 554

Leach (Ornithomyia, Ornithoctona).............. 581

Meigen ( Musca, Calliphora).. 517

erythrocera Brauer and Bergenstamm (Hes-

peromyia) ................. 551

Desvoidy. See aterrima Villiers. 524 (Athrycia).......... 512

erythrostoma Hartig ( Tachina, Hemithæa)... 550 erythrura Meigen (Sarcophaga, Servaisia).... c05 esuriens Fabricius ( Musca, Willistonia)...... $t 19$ eulophus Loew (Itippelates, Ceratobarys).... 519 eumyothyroides Townsend. See theutis

Walker........................ 537 euphemoidea Desvoidy. See pertusa Meigen. 550 exile Coquillett (Clytiomya, Eutrixa)........ 543 exilis Coquillett (Ccratopogon, Atrichopogon). 512

(Metacosmus)........... 568

Meigen (Agromyza, Agrophila)........ 504 (Drapetis) ............... 535 eximia Stenhammer (Colopa, Fucomyia).... 545

Wiedemann ( Trypeta, Hexachaeta)... 552 exoleta Meigen (Tachina, Entomophaga)..... 538 exquisitus Meigen (Dasypogon, Habropogon). 548 exsurda Pandelle (Limnophora, Melanochelia) ............................ 566 fasciata Desvoidy. See nigripes Fallen..... $\quad 530$ See octopunctata....... 572 See pulchella Rossi....... 615 (Ramonda)............... 599 Egger (Fallenia, Eggeria).......... 536 Guerin (Caloptera) ............... 517 (Caloptera, Evanioptera)..... 543

Kieffer (Dichrodiplosis) ............ 533

Macquart (Didea)............... 533

(Didea, Enica)........... 538

Meigen (Ceratopogon, X ylocrypta)... 621 (Macrocera, Geneja)........ 546 (Mycetobia, Ditomyia)...... 534 (Mycetophila, Rymosia)..... $\quad 602$

(Myopa, Ischiodonta)....... 555 (Platypeza)................ 592

(Platypeza, Clythia)....... 525

(Platyura) ............... 592

(Platyura, Zelmira) ..........

Say (Heteromyia) . . . . . . . . . . . . . 551

Westwood (A sthenia) .............. 511

(Asthenia, Blepharicera).. 514

Wiedemann (Acrochæta) ......... 502 (Gonia, Pissemya)...... 591

fasciatus Brauer (Myxosargus) . . . . . . . . . . 573

Fabricius. See calopus Meigen.... 608

Meigen (Ceratopogon, Sphaeromias). $\quad 607$ fascipennis Coquillett (Scutops)... . . . . . . 604 Fabricius (S/ratiomys, Euryneura) 543 Macquart. See coaretata Fallen. 554 See fuscipennis Say. . $\quad 577$

Meigen (Leia).................. 558 (Leia, Glaphyroptera)..... 547 (Leia, Neoglaphyroptera). $\quad 575$ Say (Limnobia, Epiphragma) ..... 539 Williston. See tibialis Walker... 510 
Page.

fascithorax Williston (Ceriogaster)

fastuosa Meigen ( Tachina, Eumetopia)......

fauna Meigen ( Tachina, Nilea)............

faustina Osten Sacken (Anthrax, Hyalanthrax)

febrilis Linnæus (Tipula, Dilophus)........ (Tipula, Philia) ..........

femoralis Desvoidy (Aciura).

Schiner (Sarcophila, Sarothromyia).

Wiedemann (Cephalia, Conopsida).. (Cephalia, $\mathbf{M}$ i c hogaster).............

femorata Fabricius (Empis, Pachymerina)...

Meigen (Ceratopagon, Ceratolophus)

(Ceratopogon, Johannseniella).

Williston. See fimbriata Water-

house....................................

femoratus Meigen (Ceratopogon, Prionomyia)

(Ceratopogon, Serromyia)..

fenestralis Fallen (Heleomyza, Aecothea).....

Linnæus ( Musca, Atrichia).......

( Musca Cona)...........

( Musca, Hypselura).....

(Musca, Omphrale).....

(Musca, Scenopinus)....

Scopoli ( Tipula, Rhyphus).......

( Tipula, Sylvicolae).......

fenestrarum Desvoidy. See flava Linnæus. . See serrata Linnæus

Scopoli. See fenestralis Scopoli.

fera Desvoidy (Fellaea)..................

Linnæus ( Musca, Eupeleteria).........

ferina Fallen ( Musca, Myocera).............

(Musca, Phorostoma)..........

ferruginea Fallen (Rhingia, Eugeniamyia)....

(Rhingia, Exocheila).......

(Rhingia, Hammerschmidtia)...

Linnæus (Conops, Cylindrogaster).

(Conops, Sicus).........

Macquart (Conops, Conopoideus).

(Odontomera).........

(Odontomera, Cyrtometopa)................

See trimaculata Fabricius. . . . .............

Meigen (Linnobia, Phylidorea)....

Scopoli ( Musca, Coenomyia)...... ferrugineus Fabricuis (Hybos, Macrostomus).

Walker. See annulata Meigen.. ferruginosa Meigen (Mycetobia, Diadocidia)..

( Mycetobia, Macronevra)... festæ Giglio-Tos (Pityocera) .............. festina Meigen. See elegans Meigen......... festiva Desvoidy (Guerinia) ...............

(Guerinia, Himera)........ (Guerinia, Stægeria) .......

Loew (Trypeta, Euaresta).......... festivus Loew (Gaurax)................... filicis Felt ( Cecidomyia, Lobopteromyia)...... filiformis Fabricius. See corrigiolata Linnæus filipes Fabricius ( Tipula, Maekistocera).....
Page.

fimbriata Waterhouse (Anapera, Brachypteromyia).

516

Wulp (Brachicoma, Thysanomyia). 615

fimetaria Linnæus ( Musca, Oblicia)......... 577

(Musca, Pelethophila)..... 586

( Musca, Psila)........... 596

(Musca, Psilomyia)....... 597

fitchii Osten Sacken (Protoplasa)........... 595

(Protoplasa, Idioplasta). $\quad 555$

flabellifer Osten Sacken (Polymedon) ........ 594

flava Coquillett (Phasiops) .............. 588

(Zagonia) ................ 621

Desvoidy. See flava Linnæus........ 562 See rorida Fallen........... 563

Fabricius. See obsoleta Fallen....... 602

Linnæus ( Musca, Chyromya) ......... 524

(Musca, Lisella).............. 562

(Musca, Scyphella).......... 604

(Musca, Thyrimyza).......... 615

Meigen (Heteromyza, Clusia)......... 525

(Heteromyza, Macrochira)....... 564

(Heteromyza, Peratochetus)..... 587

(Heteromyza, Stomphastica)..... 609

Schellenberg. See fimetaria Linnæus.. 586

Stæger (Boletina, Cœlosia) ........... 526

Townsend (Clytia, Euclytia).......... 541

See venatoris Coquillett..... 574

Winnertz (Macrorrhyncha) ............ 564

flaveola Coquillett (Megaparia, Eumegaparia) 541

Desvoidy (Lentiphora)............ 559

Fabricius ( Musca, Dryomyza)....... 535

( Musca, Dryope)......... 536

Fallen (Phytomyza) ............... 590

flavescens Desvoidy. See aurea Fallen...... 560 (Phoræa) ............ 588

Johnson (Sepsisoma)............. 605

Linnæus ( Tipula, Limnoica)...... 561

flavianalis Macquart (Erax, Eichoichemus).. 537

flavicans Desvoidy. See wiedemanni Mei-

gen .......................... 580

Ruthe. See ferruginosa Meigen.... 532 flaviceps Meigen (Notiphila, Hydrellia)...... 553 (Notiphila, Hydropota)..... 554

Zetterstedt (Gonia, Onychogonia)... 579 flavicinctus Meigen. See occulatus Fallen.. 532 flavicollis Meigen (Porphyrops, Achalcus) ... 502 flavicornis Desvoidy. See flava Linnæus.... 604

$$
\text { (Lythia)............. } 563
$$

flavicosta Wulp (Rhombothyria)........... 600 flavidus Coquillett (Parepalpus) ............ 585 flavipalpe Zetterstedt (Rhaphium, Thinophilus) ............................ 614

flavipalpis Desvoidy (Belida)............. 513

flavipalpus Marquart (Hystricia, Bombylio-

myia)................................ 515

flavipennis Coquillett (Pycnoglossa) ........ 598

Desvoidy. See radicum Fabricius....................... 539 Fallen ( Iusca, Pachystoma)..... 582 Osten Sacken (Sigmatomera)..... 605 Williston. See insolita Walker.. $56^{\circ}$ 
flavipes Bigot (Homodexia, Eucalodexia)..... Desvoidy (Hebia) .................. Fallen (Notiphila, Philygria)......... Gray (Pterodontia) ................. Linnæus (Conops).................. Macquart. See flavipes Meigen..... (Campeprosopa).......... (Mycetophila, Mycetina) .. (Mycetophila, Mycozetæa).

Meigen (Campylomyza).............

(Ceratopogon, Alasion)........ (Ceratopogon, Palpomyia).... See minimus Becker......... (Ocydromia, Lemtopeza)..... (Ceratopogon, A pogon).......

Panzer (Syrphus, Cheilosia)......... flavitarsis Meigen (Chlorops, Cryptonevra).... flavitibia Walker (Ubristes)................ flaviventris Macquart. See marginata Say..

Meigen (Porphyrops, Anepsiomyia)................. (Porphyrops, A nepsius).. flavoferruginea Osten Sacken (Cladura)...... flavum Macquart (Heterostylum)............ flexa Wiedemann ( Trypeta, Tritoxa)......... flexivitta Walker (Bricinnia)................ floccosa Maequart (Chortophila, Erioischia)... See penicillata Rondani...

floralis Fallen ( Tachina, Meigenia).........

Meigen (A scia, Neoascia).............

florea Desvoidy. See processionex Ratzburg............................

floricola Desvoidy. See cardui Meigen...... Felt (Cecidomyia, Prodiplosis)....... . floridensis Townsend (Pachyophthalmus, Sarcomacronychia)......

(Siphophyto)...........

(Tachinophyto)........ fœrsteri Meigen. See fasciata Macquart..... foreipata Meigen ( Tachina, Dionæa) .......... formicaria Desvoidy. See rufipes Meigen.... formidabilis Bigot (Rhamphinina, Eudexia).. formosa Scopoli ( Musca, Chloromyia)........

Harris ( Musca, Epistrophe) .......... forreri Wulp (Nemoræa, Microtrichomma).... foveolata Meigen (Piophila, Stearibia)........ fragilis Desvoidy. See pallida Fabricius..... Loew (Toxorhina)

fragillima Westwood (Mongoma)............ fraterculus Wiedemann (Dacus, Acrotoxa).... frauenfeldi Schiner (Thalassomya)........... frenchii Williston (Masicera, Achætoneura).. frigida Fabricius. See eximia Stenhammer.. See pilipes Haliday.

frontalis Loew (Chrysogaster, Campineura)... Macquart (Chortophila, Trigonostoma) ........................

Meigen (Tetanocera, Oxyrhina)...... ( Tetanocera, Trigonometopus) frontosa Hine (Akronia) ................... fuciformis Fabricius (Syrphus, Mallota)...... fucorum Fallen (Scatomyza, Halithea)........ fulgens Meigens. See comta Fallen .......... fulminans Fabricius. See semilurtifera Vil-

liers...
Page.

540

549

588

598

527

558

512

572

572

518

504

583

505

558

509

521

528

618

527

506

506

524

552

617

516

539

579

566

574

582

531 fulvicollis Fabricius (Hirtea, Plecia)......... 592

Walker (Rachicerus)............ 599

fulvicornis Desvoidy. See cinerea Fallen.... 557 fulvifrons Walker (Ornithomyia, Stilbometopa) $\quad 609$ fulvipalpis Desvoidy (Pictinia)........... 591

fulvipes Cresson (Hiatus) . ............... • 552

Desvoidy (Ceranthia)............ 519

(Ceranthia, Vafrellia)..... 619

Macquart. See macellaria Fabricius....................... 584

Walker (Tabuda) ................ 611

Westwood (Chalcidimorpha)......... 521

fulvithorax Wiedemann (Pangonius, Erephop-

sis)............ 539

(Pangonius, Melpia) 567

fulviventris Bigot ( Macroceromys).......... 564

fulvohirta Wiedemann (Anthrax, Chrysan-

thrax $\ldots \ldots \ldots \ldots \ldots \ldots \ldots \ldots \ldots \ldots \ldots .523$

fulvus Wiedemann. See brevirostris Mac-

quart.............................. $\quad 606$

funditor Loew (Dolichopus, Spathichira)..... 606

funebris Fabricius ( $\mathrm{Musca}$, Drosophila)...... 535

Meigen. See grossipes Linnæus.... 553

funesta Desvoidy (Cerophora)............. 520

funestus Harris. See pomonæ Fabricius.... 598

Osten Sacken (Epibates)........... 538

fungorum De Geer ( Tipula, Exechia)......... 544

Desvoidy. See rufa Fallen....... 610

furcata Say (Pyropa)................... 599

furcatus Egger (Pipunculus, Prothecus)..... 595

furcifera Bigot. See curvicauda Gerstæcker. $\quad 570$

furens Poey (Oecacta) ..................... 578

fusea Giglio-Tos (Berismyia).............. 513

Macquart (Omaloce phala)............. $\quad 579$

(Omalocephala, Tylemyia)... 618

Meigen (Cecidomyia, Phyllophaga)..... 590

See hybrida Meigen........... 515

(Limonia, Numantia).......... 576

fuseata Fabricius (Tipula, A nisopus)....... 507

( Tipula, Phryne)......... 589

fuscicollis Westwood (Apiocera)............ 508

fuscicornis Meigen ( Mycetophila, Dynatosoma) 536

fuseipennis Desvoidy (Dasyna)........... 531

Lioy. See coaretata Fallen...... 554

Macquart (Ceratophya, Meso phila)............. 568

See undata Wiede-

mann.............. 582

Say (Baccha, Ocyptamus)........ 577

fuscipes Meigen (Beris, Octacantha)........ 577

fuseitarsis Say (Beris, Allognosta)........... 505

fuscogrisea Rondani (Brachyneura)........ 516

fuscovaria Osten Sacken (Limnophila, Dicra-

nophragma)........................ 533

fuscus Meigen. See fuscata Fabricius....... $\quad 507$

gagatea Desvoidy. See costata Fallen...... 619

(Freræa).............. 545

(Jurinia)............. 556

(Rhinomya)............ 600

gagatina Meigen ( Tachina, Cassidæmyia)..... 518

( Tachina, Rhinophora)...... 600

galeata Haliday (Phora, Drepanophora)..... 535

(Phora, Leptophora)......... 560

(Phora, Metopina).......... 569

galiorum Kieffer (Schizomyia)............. 604

gallica Desvoidy. See hebes Fallen....... 606 
gangrenosa Desvoidy. See crassipennis Fabricius............................ geminata Brauer and Bergenstamm. See rufilatera Rondani..............

Say (Scava, Toxomerus).

gemmicola Kieffer (Bertieria) .............. genalis Coquillett (Metaphyto)............. geneji Rondani. See melanoptera Fallen... geniculata De Geer (Musca, Bucentes)....... (Musca, Crocuta)....... (Musca, Siphona).......

Fallen ( Musca, Coenosia)......

Townsend (Coronimyia).......... gentilis Desvoidy. See helluo Fabricius.... georgiæ Brauer and Bergenstamm (Pseudogermaria). germana Osten Sacken (Dicranoptycha) germanica Desvoidy. See chalconota Meigen germinationis Linnæus ( Musca, Opomyza)... gestroi Rondani (Ornithomyia, Ornitheza).... ghilianii Rondani (Meromacrus)........... gibba Fabricius ( Musca, Curtonotum)...... (Musca, Diplocentra)...... gibbicornis Macquart (Acreotrichus)....... . gibbosa Linnæus ( Musca, Henops).......... . gibbosus Fabricius. See zonatus Erỉchson.. Linnæus (Asilus, Laphria)........ gibbus Loew (Lordotus) ... . . . . . . . . . . . . gigas Herbst ( Tabanus, Brachystomus)...... gilva Wulp (Baryplegma) ................. gilvipes Coquillett (Metapogon)

glabra Meigen (Chlorops, Chloropisca)...... (Notiphila, Athyroglossa)..... glabricula Fallen (Empis, Ocydromia)....... glacialis Pokorny (Oreomyza).............. gladiator Mik (Hypocharassus)............ globosa Fabricius (Musca, Cistogaster)...... (Musca, Pallasia)........ globosus Fabricius ( Mulio, Dimeraspis) ..... globulus Panzer (Syrphus, Acrocera)........ gnava Meigen ( Tachina, Carcelia)......... (Tachina, Chetoliga).......... goliath Brauer and Bergenstamm. See formidabilis Bigot..

goniæformis Meigen ( Tachina, Baumhaueria) ( Tachina, Pachycephala). gonioides Coquillett (Opsidia) .............. gonoides Townsend (Eucnephalia). .......... grabhamii Theobald (Anopheles, Cycloleppteron).

gracilenta Wulp (Xanthomelana).......... gracilis Fallen (Anthomyza) ............... (Anthomyza, Anthophilina).... (A nthomyza, Leptomyza)...... (Anthomyza, Psilosoma)....... Philippi (Deromyia) ................ Wiedemann (Dexia, Lepıoda) ........ . graminum Fallen (Drosophila, Scaptomyza).. ( Tachydroma, Stilpon).... gramma Meigen. See assimilis Fallen ....... grandicorne Rondani (Cryptochetum) ........ grandis Bergroth (Holorusia) .............. Osten Sacken (Bibiocephala)....... Williston (Hadromyia) ............. Zetterstedt ( Tachina, Scotia)........ grayii Theobald (Wyeomyia)

age

567

565

615

513

568

507

516

528

606

528

523

596

533

564

580

581

567

529

534

502

550

578

557

563

516

513

568

522

512

580

555

524

582 533

502

518

522

541

513

582

580

541

529

620

507

507

560

597

531

559

603

609

522

528

552

514

548

604 risea Desvoidy $(O s$

Fallen ( Musca, Neurorta)........... 576

(Tachina, Cleonice)........... 525

( Tachina, Pherecida) .......... 588

griseipennis Stannius (Dolichopus, Raghe-

neura).............................. 599

griseola Meigen. See annulipes Zetterstedt... 560

grisescens Desvoidy. See nigripes Fallen.... 563

grossa Linnæus ( Musca, Echinomya)....... 536

(Musca, Faunia)........... 544

(Musca, Larvævora)......... 557

(Musca, Tachina)........... 611

grossificationis Fabricius. See roralis Lin-

næus.............................. 567

grossipes Linnæus ( Musca, Hybos)......... 553

( Musca, Noeza).......... 576

guerini Bigot (Sepsis, Macrostenomyia)...... 564

(Sepsis, Stenomacra).......... 608

Zetterstedt. See bimaculata Schummel...

guntheri Arribalzaga (Holmbergia, Rhopalosyrphus)............ 600

(Holmbergia)......... 552

gurges Walker (Syrphus, Mesograpta)....... 568

gurgus Walker (Syrphus, Mesogramma)...... 568

guttata Fallen (Notiphila, Hyadina)........ 553

(Notiphila, Hydrina)......... 553

Haliday (Leptosceles) ............. 560

gyllenhali Zetterstedt (Anthalia)........... 507

gyrans Fallen (Agromyza, Redia)............ 599

Loew (Ortalis, Tephronota).......... 613

hæmatodes Meigen (Sarcophaga, Ravinia).... 599

hæmorrhoa Meigen (Sarcophaga, Erichsonia). $\quad 539$

(Sarcophaga, Mulsantia). 571

Rondani. See ruficornis Macquart..................... 583

Wieciemann (Laphria, Dasyllis).. $\quad 531$

hæmorrhoidalis Fabricius (Culex, Lynchiella). 563

(Culex, Megar -

hinus)........ 566

Fallen ( Musca, Bercœa)..... 513

( Tachina, Bonellia).... 515

Meigen (Cordilura, Microprosopa) ...

halictivorus Rondani. See olivaceus Loew.-

halidayi Loew (A sindulum, A ntlemon)...... 508

halteralis Coquillett (Bibioides)............ 514

hamata Fallen ( Musca, Drymeia)........... . 535

hamatus Macquart (Litarhynchus)........... 562

hamifera Loew ( Trypeta, Spilographa)...... 607

harrimani Coquillett (Ornithodes)........... 581

harveyi Townsend (Drochrta).............. 530

hastata Coquillett (Isoglossa)............... 556

haustellatus Fabricius. See mauritanus Linnæus................................ 612

hebes Fallen ( Tachina, Spallanzania)...... 606

Meigen: See bucephala............. 525

hedemanni Brauer and Bergenstamm. See cyaneiventris Macquart.................. 584 helicis Townsend (Sarcophaga, Helicobia)..... 550 helluo Fabricius (Musca, Chryseria)........ 523 (Musca, Phanigaster)....... 587 helymus Walker ( Tachina, Metachæta)...... 568 hemipterus Fabricius (Syrphus, Alophora)... 505

(Syrphus, Phasia)..... 587 
Page.

herbarum Desvoidy. See juncorum Fallen.. See syngenesiæ Fabricius................. heringi Zetterstedt (Pipiza, Heringia)........ heryngii Rondani. See impunctata Loew... hesperidarum Williston (Acroglossa)......... hesperus Brauer and Bergenstamm. See

frenchii Williston....................... heterocera Desvoidy. See costata Fallen.... heteroneura Brauer and Bergenstamm (Parahypochaeta)................ Meigen (Miltogramma, Elpigia).

(Miltogramma, Heteropterina)............... (Miltogramma, Taxigramma).............

hiemalis Desvoidy. See moerens Meigen.... De Geer ( Tipula, Petaurista)....... (Tipula, Trichocera).......

hieroglyphica Bigot. See nitidus Wiedemann ................... Wulp (Clinopera)........... hilarella Zetterstedt (Miltogra mma, Hilarella) hilaris Fallen ( Musca, Dendrophila)........ (Musca, Eustalomyia)........ hilpa Walker (Pterocosmus).............. hinei Coquillett (Meigeniella).............. hippoboscoides Aldrich. See chrysocoma

Osten Sacken........................ hirsuta Coquillett (Omomyia)............. hirsutus Townsend. See californiensis Macquart. .

hirta Bigot (Anoxycampta) ..............

(A noxycampta, Pseudoperichæta).

Meigen (Sciophila).

hirtellus Meigen. See pilosellus Loew ....... hirticeps Fallen ( Musca, Lasiops)........... hirticornis Fabricius (Chironomus, Polymera)

Latreille. See virescens Fabricius hirtipes Desvoidy (Olina)................. hirtula Zetterstedt (A ricia, Thricops)........ (Aricia, Tricophthicus).... hirtus Loew (Tetropismenus).............. hispaniense Stein. See peregrinum Meigen. hispidus Wiedemann (Dasypogon, Gonioscelis) hoffmeisteri Loew. See bipunctata Fallen. holosericeus Meigen (Pipunculus, Atelenevra). Scopoli (Styrex).................. horticola Desvoidy. See hortorum Fallen... hortorum Fallen ( Iusca, Morellia)......... . hortulana Linnæus ( Tipula, Bibio).......... Rossi ( Musca, Ceratoxys).......... ( Mrusca, Meckelia) ........... hortulans Meigen. See flavipes Meigen..... hubbardii Coquillett (Henicomyia).......... humeralis Stein (Eremomyia)............. humilis Coquillett (Parephydra)............

Winnertz (Phthinia)................ hyalomoides Townsend. See didyma Loew. hybotina Fallen (Empis, Oedalea).......... hybrida Meigen (Limonia, Helobia)..........

(Limonia, Symplecta)........ ( Macrocera, Bolitophila)..... ( Macrocera, Messala)....... hybridus Meigen (Bombylius, Geron)......... hydroleon Linnæus ( $M u s c a$, Eulalia).........

(Musca, Odontomyia)....
Page.

hydromyzina Fallen (Cordilura, Spaziphora). 607 hyoseyami Panzer ( Musca, Pegomya)....... 586 hyphantriæ Townsend (Meigenia, Hyphantrophaga).......................... 554 hypoleon Linnæus (Musca, Hermione)...... 551

(Musca, Oxycera)....... 582 hypoleuca Meigen. See coleoptrata Scopoli. 608 hystrix Brauer and Bergenstamm. See nigra Macquart...................... 584 Brues (Xanionotum).............. 620 Fabricius (Musca, Tachinodes)...... 611 iceryæ Williston (Lestophonus)............ 560 ichneumonea Linnæus ( Musca, Loxocera)... 563 illinoisensis Townsend (Eumyothyria) ...... 542 illucens Linnæus ( Musca, Hermetia)........ 551 illustris Desvoidy. See lefeburei Desvoidy.. 541 imbecillus Loew (Spodius) .............. 607 imberbis Fallen (Bibio, Psilocephala)........ 597 imbuta Wiedemann (Lauxania, Griphoneura) 548 immaculata Desvoidy. See albicollis Meigen 574 Haliday (Heleodromia)........ 549

(Heleodromia, Microcera) .............. 569

(Heleodromia, Sciodromia).............. 604

Macquart (Cistogaster, Gymnoclytia) $\ldots \ldots \ldots \ldots \ldots \ldots \ldots \ldots .548$ Meigen (Limonia, Bophrosia).... 515 (Limonia, Tricyphona).. 616 impatiens Desvoidy. See costata Fallen.... 518 imperialis Desvoidy (Peckia) ............. 585 (Peckia, Phrissopodia)... 589 impuncta Fallen (Musca, A ricia).......... 510 ( Musca, Yetodesia)........ 621 impunctata Loew (Tetanops, Terelliosoma).. 613 inaequipes Bigot (Cholomyia) ............ 522 inamøna Walker (Solva)................. 606 inanis Fallen ( Tachina, A nthoica).......... 507 (Tachina, Chremia).......... 523 (Tachina, Myobia)............ 572

(Tachina, Solieria)............ 606 incana Fallen (Tachina, Tenuicera).......... 612 inchoata Melander (Thinodromia).......... 614 incisuralis Macquart. See puella Wiedemann 512 incompletus Macquart (Pangonius, Diclisa). 533 (Pangonius, Scione).. 604 incrassata Meigen (Phora, Nemosia)......... 574 incurva Fallen ( $P$ silopa, Discomyza)....... 534 indagator Loew (Cacoxenus). ............. 516 indecisa Townsend (Pseudomyothyria)....... 596 inepta Meigen ( Tachina, Elpe)............ 537 inermis Bigot (Blepharipeza, Parachaeta).... 583 Wiedemann (Cyphomyia, Chordonota) ............ 522 (Sargus, Histiodroma) 552 inflatus Loew (Pithogaster)............... 591 inflexa Wulp (A nisia) .................. 507 infuscata Desvoidy. See segregata Rondani. $\quad 536$ Meigen (Rhamphomyia, Enicop-

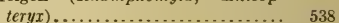
innoxia Desvoidy. See fauna Meigen......... 576 insolita Melander (Lactistomyia)............ 557 Walker ( Tachina, Atropharista)...... 512 (Tachina, Melanophrys)..... 567 intermedia Townsend (Sarcotachinella)..... 603 interrupta Mulsant. See rutila Wiedemann. 617 interruptus Macquart (A silus, Eristicus).... $\quad 540$ (A silus, Neoëristicus).. 574 
Page.

intestinalis De Geer (Oestrus, Enteromyza).. 538 (Estrus, Gasterophilus) 546 (Oestrus, Gastrus)...... 546 inulæ Desvoidy. See ephippium Fabricius.. $\quad 575$ inusitata Melander (Coloboneura).......... 526 iopterus Wiedemann ( $A$ silus, Anarmostus).. irritans Fabricius. See geniculata De Geer.. Fallen ( Iusca, Psiloptera)............ Linnæus (Conops, Hæmatobia)....... (Conops, Lyperosia)........ (Conops, Priophora)......... irrorata Coquillett (Traginops).............. Fallen ( Tachydromia, Ardoptera).... ( Tachydromia, Dolichocephala) ( Tephritis, Campiglossa)....... italica Costa ( $A$ delina) jacchus Fabricius (Anthrax, Argyrospyla)... jaculus Fallen (Hydrophorus)................ (Hydrophorus, A northus)...... (Hydrophorus, Orthobates)..... (Hydrophorus, Tæchobates).... jænnickeana Osten Sacken (Isopenthes)...... jamaicensis Theobald (Culex, Feltidia)....... (Culex, Grabhamia)... javensis Doleschall. See acrostacta Wiedemann............................. johnsoni Coquillett (Exepacmus).........

(Exoristoides) ........... (Hesperodes)...........

(Lasioneura)............

(Stenoxenus) ...........

Darlington (Porsenus) ............. juncorum Fallen (Ochtiphila, Estelia)....... juncta Coquillett (Roederiodes)............. juniperina Felt (Walshomyia) .............. Linnæus ( Tipula, Oligotrophus).. urinoides Townsend. See insolita Walker. kansensis Aldrich ( Metapelastoneurus)....... Townsend. See varia Wulp....... karnerensis Felt ( Cecidomyia, Odontodiplosis) lacteata Townsend (Euomogenia)............ lacteipennis Fallen (Agromyza, A nisonevra).. Loew (Lobioptera, Ophthalmomyia).................................

Zetterstedt (A ricia, Dexiopsis).. lacustris Scopoli ( Musca, Alloeoneurus)...... (Musca, Orthoceratium)... laeta Fallen ( Musca, Euphemia) ........... Meigen (Chlorops) ................... (Chlorops, Titania).............. (Tachina, Frontina) ........... lanipes Fabricius ( Thereva, Galactomyia).... laniventris Eschscholz (Empis, Eriogaster)... lappona Linnæus ( Musca, Cinxia).......... (Musca, Sericomyia)....... lapponicus Zetterstedt (Nephrocerus)........ larvarum Linnæus ( Musca, Eutachina)..... . ( Musca, Exorista)........ lasciva Fabricius ( MFusca, Grallopoda)..... . . lasiophthalma Macquart (Chortophila, Dasi-

opa) ............................. lasiophthalmus Macquart (Pangonius, Clanis) lata Coquillett (Condidea). lateralis Desvoidy (Arisbæa)............. 510

See rudis Fallen........... 583

Fallen (Ocyptera, Besseria)........ 513

(Ocyptera, Wahlbergia)........ 619

Loew (Hecamede, Allotrichoma)..... 505

lateritia Loew (Dirhiza)................. 534

laticauda Loew (Steneretma).............. 608

laticornis Meigen. See lineata Fabricius..... 520

(Lonchæa, Teremyia)........ 613

(Sarcophaga, Sfaerogaster).. 605

latifrons Fallen (Musca, Sarcophila)......... 602

Hough (Calliphora, Eucalliphora)... 540

latipennis Fallen (Dolichopus, Hygroceleuthus) 554

latipes Meigen (Agromyza, Macrurus) ........ 564

llatisquama Coquillett (Tinolestes).......... 615

atreillei Desvoidy (Mesembrina, Eumesem-

brina) ............................ 541

lauta Loew (Triptotricha) ................ 617

lefeburei Desvoidy (Echinomya, Eudora)..... 541

(Echinomya, Eudoromyia) 541

leonum Westwood (Stylomyia)............ 610

lepidota Wiedemann. See crassipes Fabricius $\quad 559$

lepidotus Wiedemann. See crassipes Fabricius.............................. 549

leptiformis Fallen (Heteroneura) ............ 551

(Heteroneura, Callomyza). 517

leptogaster Panzer ( Musca, Chyliza)........ 524

leucocephala Rossi ( Mfusca, A raba)......... 509

( MIusca, A rabella)........ 509

( Mrusca, Argyrella)....... 509

(Musca, Argyria) ........ 510

(Musca, Metopia)........ 569

leucophrys Wiedemann (Tachina, Blephari-

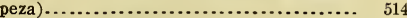

leucopogon Wiedemann (Pangonius, Fidena). 545 leucoprocta Wiedemann (Mulio, Ogcodocera) 578

leucoptera Johnson (Dichaetoneura)......... 532

leucostoma Loew (Amiota)................ 505

Wiedemann (Anthomyia, Ophyra) 58J

leucozona Bilimek (Pholeomyia).......... 588

libatrix Panzer ( Musca, Atilia) ............. 512

(Musca, Clemelis)........... 524

(Musca, Myxexorista)........ 573

(Musca, Sagaris)............. 602

(Musca, Zelinda)........... 621

(Musca, Zenillia) ............ 621

ligniperdæ Brauer and Bergenstamm ( $X$ ylotachina) ............................. 621

limbata Loew (Rhamphomyia, Megacyttarus). $\quad 566$ limbipennis Macquart. See fasciata West-

wood............................. 514

limosa Fallen (Copromyza, Nerea).......... $\quad 575$

lineata Fabricius ( Musca, Oscinis)......... 581

(Rhingia, Eurimyia) ....... 542

(Tipula, Cerotelion)........ 520

Fallen (Musca, Listeria)............. 562

lineatus Aldrich (Pelastoneurus, Sarcionus).. 602

litorella Fallen (Ochtiphila, Litorella)........ 562 (Ochitiphila, Schoenomyza)... 604

littoralis Desvoidy. See vespertina Fallen.. 554

litturata Olivier (Ocyptera, Euantha)....... 540

lituratus Loew (Pterallastes, Teuchocnemus). $\quad 613$ 
Page.

livens Fabricius ( Musca, Hydromyza)..... ( Musca, Nupharia)........ lobata Felt (Cecidomyia, Hyperdiplosis)..... (Mycodiplosis, Coquillettomyia). locuples Desvoidy. See cyaneus Fabricius. loewi Becker (Pselaphephila) ............. lonchæoides Zetterstedt (Earomyia)........ loncheus Rondani. See lasiophthalma, Dasi-

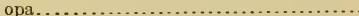
longibarbus Loew (Cyrtopogon, Eupalamus). longicollis Loew (Cecidomyia, Asynapta).... . longicornis Bigot (Stictomyia).

Fallen (Hydrochus)............. (Tachina, Fallenia)....... (Tachina, Frivaldskia)....

Schiner. See distincta Meigen... longimana Fallen (Tanypeza) ............. longipennis Fabricius ( Musca, Minettia).... Wiedemann (Trypeta, Strauzia) longipes Fabricius ( Musca, Cardiacephala). longirostris Hardwicke (Pangonius, Nuceria).

Meigen (Limnobia, Helius)....... (Limnobia, Megarhina).. (Limnobia, Rhamphidia). ( Tachina, Aphria)....... ( Tachina, Olivieria)..... (Tachina, Rhynchosia)...

Theobald (Wyeomyia, Phoniomyia)......................

Wiedemann (Limnobia, Leptorhina).................... ongiseta Meigen (Heleomyza, Eccoptomera)..

Wiedemann (Dexia, Chaetona) ..... longiungulatus Macquart (Dasypogon, Ma-

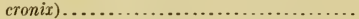
longiventris Loew (Sybistroma, Hercostomus) longurio Loew (A trichia) ................. (Atrichia, Pseudatrichia)..... loti De Geer ( Tipula, Contarinia)........... loxocerata Fallen (Cordilura, Hexamitocera). lucens Townsend (Drepanoglossa).......... lucida Fallen ( Tephritis, Myoleja).......... Gerstaecker (Panacris) ..............

Meigen ( Tachina, Hyalurgus) ........ lucifera Dahl (Puliciphora)................ (Puliciphora, Stethopathus).... lucorum Linnæus ( Musca, Leucozona)....... ( Musca, Syrphus)........ luctuosa Desvoidy. See longipennis Fabricius.........................

Meigen ( Tachina, A medoria) ........ luctuosus Meigen (Dasypogon, Saropogon)... ludibunda Desvoidy (A minta) ............. lugens Wiedemann (Mycetophila, Allodia)... lugubris Desvoidy. See umbrarum Fabri-

cius..........................

Winnertz (Asynapta, Winnertzia). . lunata Theobald (Wyeomyia, Lesticocampa). lundibunda Desvoidy. See quadripustulata

Fabricius . . . . . . . . . . . . . . . . . . . lupina Williston (Brachymyia)........... lurida Meigen (Eurina).................. luridum Rondani (Blepharepium)......... luridus Walker (Pangonius, Lilæa). lusitanicus Wiedemann ( Mydas, Leptomydas)
aiglio-Tos (Molynocoelia) Meigen (Erioptera)

(Erioptera, Polymeda)........ 593

(Limonia, Furcomyia)......... 546

( Macrocera).................. 564

( Macrocera, Euphrosyne)...... 542

Panzer (Lonchoptera)................ 562

(Lonchoptera, Dipsa)........ 534

luteipes Williston (Scoliopelta)............. 604

luteofusca Rondani (Dasineura).......... 530 luteola Coquillett (Belvosia, Goniomima).... 547

Gmelin ( fusca, Myolepta).......... 573

lutescens Rondani. See flava Meigen. ... . . . 587 Iyrata Williston (Dichocera).............. 533 mabelæ Melander (Ragas, Hesperempis).... 551 macellaria Fabricius ( Musca, Paralucilia).... 584 macilentus Wiedemann (Systropus)......... 611 (Systropus, Cephenus).......... 519

macquarti Stæger ( $A$ tomogaster) ......... 512 Zetterstedt (Iteaphila)........... 556 Zetterstedt (Iteaphila, Steleocheta). $\quad 608$ nacrocephala Giglio-Tos (Camerania)..... 517 macrocera Say (Limnobia, Lasiomastix)..... 558 macrocerum Meigen (Rhaphium)......... 600 macroporum Macquart (Plinthina)........ 592 macroptera Maequart (Limnobia, Macroptera) 564 (Limnobia, Ula) ....... 618

Philippi (Idioneura) ............ 555 macrosoma Wulp (Charadrella)........... 521 maculata Coquillett (Pseudolfersia) ........ 596 Dufour (Drosophila, Leucophenga). $\quad 560$ Giglio-Tos (Paragorgopis)......... 584 Macquart. See irrorata Fallen.... 535 Meigen (Dixa) ................. 534

See melancholia Harris.... $\quad 576$ (Milichia, Alticomerus)..... 505 (Milichia, Odinia)........ 578 (Rhipidia)............. 600

Scopoli (Musca, Curtonevra)...... 529

( Musca, Graphomya)..... 548 maculatus Doane (Polyangaeus).......... 593

Fabricius (Asilus, Promachus).... 595 (Asilus, Telejoneura)... 612 (Asilus, Trupanea)..... 618

Meigen (Xylophagus, Subula).... 610 (Xylophagus, X ylomya).. 621

Rossi (Asilus, Toxophora)....... (615 maculipennis Macquart. See canariensis Bergroth.................... 509

Walker ( Tachydromia, Phoneutisca) ..................... 588 maculipes Theobald (A rribalzagia) ......... 570 magna Theobald. See eancer Theobald..... 515 magnicornis Townsend. See singularis Townsend.......................... 557 magnifica Schiner (Sarcophila, Wohlfahrtia).. 620 magnus Walker (Cyrtus, Pialoidea)......... 590 major Brauer and Bergenstamm. See hirta

Bigot..................... 596

Linnæus (Bombylius).............. 515 majorina Wulp (Myothyria) . . . . . . . . . . . 573 majuscula Wulp (Calodexia) ............ 517 malleola Bigot. See robusta Wiedemann.... 607 mallophorides Walker ( Morimma)......... 571 
Page.

manicata Fabricius. See mantis De Geer.. manicatus Meigen (Dasypogon, A nisopogon).

(Dasypogon, Heteropo-

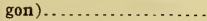

mantis De Geer ( Musca, Macrochira).......

( Musca, Ochthera) .........

mantispa Meigen. See melanocephala Fabri-

cius........................

Panzer. See melanocephala.......

marginalis Wiedemann. See regalis Des-

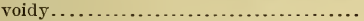

marginata Fabricius (Bibio, Ibisia) ..........

Loew (Anorostoma).............

Meigen (Sciophila, Mycomya).....

( Tachina, Klugia).........

( Tachina, Ptilopareia).....

Say (Ortalis, Idana) . . .............

(Volucella, Copestylum) ......

marginatus Linnæus. See pantherina Lin-

næus........................... marginellus Fabricius (Asilus, Ommatius).. maritima Haliday ( Halithea, Fucellia)...... Röder (Melanochelia, Neolimnophora) ...................... marklini Zetterstedt ( Tachina, Nowickia).... maroccanus Fabricius ( $A$ silus, Pogonosoma). m-atrum Meigen (Agromyza, Desmometopa). maura Fabricius (Empis, Hilara). mauritanus Linnæus ( Tabanus, Tanyglossa). maurus Mikan. See virescens Fabricius..... mediopunctatus Theobald (Cycloleppteron,

Nototricha) . . . . . . . . . . . . . . . . . . mediovittata Coquillett (Stegomyia, Gymno-

metopa).......................... meditabunda Fabricius ( Musca, Myospila).. medorina Schiner. See amedoria........... megacephala Loew. See macrocephala Giglio-Tos.

meigeni Loew ( Trypeta, Zonosema)......... melæna Meigen (Atherix, Symphoromyia).... melancholia Harris (Sylvicolx, Atherix)...... (Sylvicolæ, Nodutis)...... melanderi Brues (Acontistoptera) ........... melania Meigen (Dexix, Trisonevra)......... melanocephala Fabricius (Empis, Chelipoda).

(Empis, Hemerodromia)..........

(Empis, Lepidomya)............

(Empis, Phyllodromia)............

(Empis, Thamnodromia).........

Meigen (Tachina, Novia).....

( Tachina, Phyto)....

( Tachina, Savia).....

melanoptera Fallen ( Musca, A nthracomya)..

( Musca, Morinia)......

Hendel (Camptoprosopella)....

melanopyga Wiedemann (Pangonius, Phara)

melanura Loew ( Trypeta, Acidigona)........

Meigen (Sarcophaga, Bellieria).....

(Sarcophaga; Calyptia).....

(Sarcophaga, Scaligeria)...

(Tachina, A postrophus)...

Stæger (Mycetophila, Trichonta)...
504

507

551

564

577

559

550

598

555

507

572

557

598

555

527

574

579

545

575

576

593

532

552

612

527

576

548

573

505

517

622

610

511

576

502

617

522

550

559

590

614

576

590

603

507

571

518

587

502

513

517

603

509

616 melas Bigot. See analis Schiner.......... Page.

(Myelaphus) ............... 572

melissopodis Coquillett (Plectops) .......... $\quad 592$

mellina Linnæus ( Musca, Melanostoma) .... $566^{\circ}$

mentalis Coquillett (Tachinopsis).......... 611

merdaria Fabricius ( Musca, Scathophaga)... 603

(Musca, Scopeuma)...... 604

meridiana Linnæus ( Musca, Mesembrina).. $\quad 568$

(Musca, Metamesem-

brina) ............... 568

meridianus Rondani (Agelanius) . ......... 504

meridionalis Desvoidy (Faurella).......... 544

(Stephania)......... 608

meromelas Dufour. See pachygaster Fallen. $\quad 575$

metallica Bigot (Eurhinomallota).......... $\quad 542$

Townsend. See atra Desvoidy... . 587

(Phosococephala)...... 589

metathesis Loew (Rhaphium, Syntormon)... 611

meteorica Linnæus ( Musca, Hydrotæa)...... 554

metopia Brauer and Bergenstamm (Myio-

pharus) ...........................

metraloas Meinert (Miastor) ...............

mexicana Bellardi (Beris, Oplachantha)......

Bigot (Ozodiceromyia)...........

Brauer and Bergenstamm. See

barbata Bigot................

Brauer and Bergenstamm. See calogaster Bigot................

Brauer and Bergenstamm (Gædiopsis) .......................

Brauer and Bergenstamm (Gædiopsis, Poliophrys) .............

Brauer and Bergenstamm ( Microchira)....................

Wheeler (Sciodromia, Litanomyia). mexicanus Macquart (Helophilus, Asemosyr-

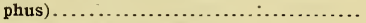
micans Erichson (Ocnæa)...............

Meigen (Pangonius, Tacina) .....-.

microcera Desvoidy ( Macquartia, Bebricia). 61 See rudis Fallen....... 540

mikii Strobl (Platycœnosia)................. 592 (Platyconosia, Choristomma).... 523

Williston (Atomosia, Atonia)......... 512 milesiformis Fallen. See scita Harris...... 570, 617 minimus Becker (Pipunculus, Alloneura)... 505 minos Meigen (A nthrax, Defilippia)......... 531 minuta Williston (Townsendia) . . . . . . . . . 615 minutus Loew. See papatasii Scopoli...... 549 mira Bigot (Enoplempis) ................... 538

Coquillett (Eusiphona)................ 543 mirabilis Giglio-Tos (Ostracocœlia)......... . 582 Osten Sacken (Diotrepha)......... 534

Townsend (Acronarisía)......... 503

(Ichneumonops)....... 555 miscella Coquillett ( Trichophora, Spanipalpis) 606 mixtus Loew (Dasypogon, Pycnopogon)...... 598 modesta Bigot (Heligmonevra)............ 550 Meigen (Limnobia, Dicranomyia)... 533 Osten Sacken (Plectromyia)........ 592

Williston (Orthoneuromyia)....... 581

(Trichopteromyia)....... 616 modestus Loew (Leptochilus).............. 559 (Leptochilus, Epacmus)..... 538 modulata Wulp (Paramintho)............ 584 mœchus Loew (Tachytrechus, Macellocerus). 563 
Page.

mœrens Bigot. See costalis Gerstæcker..... Meigen ( Tachina, Clista)........... ( Tachina, Kirbya)......... molestus Costa. See papatasii Scopoli....... mollicula Fallen ( Musca, Oplogaster)....... mollissima Haliday (Cœlomyia)........... See macroptera Macquart molobrina Rondani (Mimosciara).......... ( Mimosciara, Furcinerva) (Mimosciara, Yposatoea) monilis Linnæus ( Tipula, Ablabesmyia).... monostigma Meigen. See præcatoria Fallen. montana Osten Sacken (Limnoplila, Dactylo-

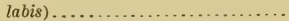
Pokorny (Chirosia, Rhadina)...... montigena Hunter (Pyritis). mordellaria Fallen ( Trineura, Hypocera)... morio Fabricius (Stomorys, Hyperalonia).... Linnæus ( Musca, Hemipenthes)......

Meigen ( Myopa, Fairmairia)......... morosa Winnertz (Syntemna) ............ mortuorum Linnæus (. Fusca, Cynomya).... (Musca, Cynophaga)... mucida Giglio-Tos (A crotoxa, Polionota).... multifasciata Loew ( Trypeta, Oedaspis)... multipunetata Rondani (Heteropterina, Metopilla)....

multisetosa WVulp (Lasiona)... murorum Rondani. See subsultans Fabricius..

mus Bigot (Rhabdopselaphus)...

Osten Sacken (Triodites)...

musca Desvoidy (Phorbia)........

muscaria Fallen (Heteroneura, Cnemacantha). 'Thomson (Glaurotricha)..

muscarius Fabricius (A silus, Acromyia).... (Asilus, Pterospilus).... muscidea Desvoidy. See rudis Fabricius. musciformis Rondani. See subcoleoptrata

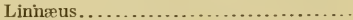
muscinum Wulp (Tyreomma).

musicus Say. See posticatus Wiedemann. mutabilis Linnæus (Musca, A phritis).......

(Musca, Microdon)...... myopæformis Röder (Eurycephala)..

(Eurycephala, Eurycephalomyia).......... myopiformis Desvoidy (Orbellia).......... myrtilli Macquart (Euthyneura) ........... mystaceum Macquart (Spogostylum) ....... nana Coquillett (Mancia) . .............. Loew (Bolbomyia) .................. nasica Haliday (Ephydra, Canace) ......... Williston (Ophromyia).

(Ophromyia, Megametopon) nebulosa Coquillett (Araba, Sphenometopa). (Cladochaeta)...........

(Pseudiastata).........

(Sciasma)............. nebulosus Walker (Diomonus)............ nemea Meigen ( Tachina, Blepharidopsis).... nemestrin? Brauer and Bergenstamm. See

aberrans Rondani. .................... nemoralis Meigen (Mycetophila, Anaclinia) .. nemorum Meigen (Tachina, Fausta)........ neomexicana Melander (Empis, Toreus)..... neomexicana Townsend (Pseudatractocera). .

De Geer (Nemotelus, Astoma) ....... 511

Loew (Coniceps) ................. 526

Williston (Gastrops) ............... 546 nigerrimus Haliday (Borborus, Elachisoma).. 537 nigra. Coquillett (Pseudapinops) ........... 595

La reille (Hexatoma) . . . . . . . . . . 552

Li næus ( Tipula, A nomaloptera)..... 507

Macquart. See acuticornis Meigen... 504 See disjuncta Wiedemann. $\quad 570$ See spuria Fallen........ 514

(Brachystylum).......... 516

(Trichophora) ............. 616

(Trichophora, Paragymnom-

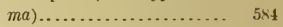

Meigen (Clinocera) .................. 525

(Clinocera, Atalanta).......... 511

(Spania).................... 606

Olivier (Leptocera) ................. $\quad 559$

Theobald (Myzorhynchella).......... 573

Townsend (Eutrichopoda)........... 543

Wiedemann (Dolichomyia).......... 535

(Limnobia, Eriocera) ..... 539

(Sciara, Odontonyx)...... 578

(Sciara, Phorodonta)...... 589

Williston (Paramyia) ............... 584

(Platophryma)............ 591

nigriceps Loew (Phora, A phiochaeta)....... 508 nigricornis Desvoidy. See errans Meigen ... 615 nigrifemoratus Macquart (Borborus, Isogaster) 556 nigrimana Loew (Brachystoma, Blepharoproc-

ta) $\ldots \ldots \ldots \ldots \ldots \ldots \ldots \ldots \ldots \ldots \ldots \ldots$

Zetterstedt (Cordilura Acanthoc-

nema)................... 502

nigripennis Brauer and Bergenstamm (Gym-

nophania)................. 548

Hough. See regalis Desvoidy... 583

Meigen (Dasypogon, Holopogon).. 552

(Laphria, Ropalocera).... 601

Wulp (Comyops).............. 526

nigripes Desvoidy. See affinis Fallen...... 553

Fabricius (Musca, Chrysotus)....... 524

( Musca, Thelairia)....... 614

Fallen ( Tachina, A netia).......... 506

(Tachina, Blondelia)......... 515

(Tachina, Cyrillia).......... 530

(Tachina, Gervasia).......... 546

(Tachina, Lydella)........... 563

(Tachina, Picconia)......... 591

Meigen. See ambigua Fallen....... 504 nigriventris Meigen (Sarcophaga, Pierretia).. 591 nigrocœruleus Latreille (Ortochile) ......... 581 nigrotæniata Bezzi. See röderi Girschner... 532 nigrum Latreille (Asindulum)............. 511 nitens Bigot (Lycastrirhyncha) ............. 563 (Orinthomyia, Orinthopertha).. 581 nitida Desvoidy (Ateria)................ 511 See quardripustulata Fabricius................... 535

Macquart (Microcheilosia) ............ 569 nitidicollis Meigen (Leia, Acnemia)......... 502 nitidifacies Hine (Nigrasilus) ............. 576 nitidiventris Wulp (Gymnomma).......... 548 nitidula Fallen (Notiphila, Ephygrobia)...... 538 (Notiphila, Hygrella)........ 554 
Page.

nitidula Fallen (Notiphila, Psilopa)......... 597

Meigen. See devia Fallen.......... 515

Wulp (Sphaerina)............. 607

nitidulus Fabricus (Bombylius, Anastœechus). $\quad 506$ nitidus Meigen (Borborus, Fungobia)........ 545

Wiedemann (Chrysogaster, Cryptineura) .......................

nivipes Theobald (Trichoprosopon).........

(Trichoprosopon, Joblotia).

nobilis Desvoidy (Purpurella)............. noctiluca Linnæus ( Musca, Pipiza).......... nodicornis Osten Sacken (Triogma, Liogma). nodulosa Macquart (Erioptera, Ormosia).... notabilis Skuse (Isoplastus).

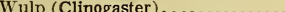

notata Fabricius ( $\mathrm{Musca}$, A noplomerus).... Linnæus ( Tipula, Ceria)............ (Tipula, Scathopse) ........ Stenhammar. See spilota Curtis.... Wiedemann (Ceratophya)............ (Ortalis, Euxesta)........ notatus Macquart. See geminata Say....... Stannius (Ammobates) .............. (A mmobates, Stannia)..... (Ammobates, Tachytrechus) ................... nova Rondani (Thrycolyga)............... nubila Meigen ( Tachydromia, Tachypeza)... nubilipennis Wulp (Acronacantha)......... nubilis Rondani ( Melanogaster)............. nuda Townsend (CEdemasoma) ............. nudicornis Schummel. See replicata Lin-

næus.............................. nudiusculus Loew (Psilocurus).

nympharum Rondani (Tachina, Microta-

china) ..............................

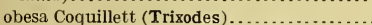

Felt (Cecidomyia, Paradiplosis)........ obesus Fabricius (Syrphus, Ornidia)......... obliqua Macquart (Oscinis, Oscinimorpha)... Say (Scæva, Ailograpta).............

( Trypeta Tomoplagia)..........

(Trypeta, Plagiotoma)........... obliterata Zetterstedt (Hormopeza).......... obscura Bigot (Physecrania)................ Brauer and Bergenstamm (Tetrachæta, Tessarochæta).

Coquillett (Arctobiella)

(Hemeromyia) .............

(Misgomyia) .............

Desvoidy (Arina) ...................

Fallen (Leptis, Ptiolina)............

( Tachina, Campylocheta).....

Jænnicke (Rondania) ...............

(Rondania, Neorondania).

Meigen. See hamata Fallen........

Wiedemann (Anisomera)............

(Hirmoneura).......... obscurella Fallen (Geomyza, Diastata)........ (Notiphila, Clasiopa)....... ( Notiphila, Discocerina)... (Phytomyza, Chromatomyia $). . . \ldots \ldots \ldots \ldots$. obscuripennis Meigen (Phania, Evibrissa)... obscurus Philippi (A nypenus).

Wiedemann (Sargus, Merosargus). obsoleta Fallen (Sapromyza) ................

(Sapromyza, Polionoma)...... 593

Meigen ( Tachina, Bellardia)......... 513

(Tachina, Maravigna)...... 56.

obtusa Loew (Lasioptera, Choristoneura).... 523

obumbrata Loew (Eudicrana).............. 541

Wulp (Hypostena, Ptilodegeeria). $\quad 598$

occidentalis Coquillett (Metaplagia) ....... 568

(Eugnoriste).......... 541

Williston (Pelomyia)........... 586 occidua Fabricius ( Musca, Sarcophagula).... 602 occlusa Desvoidy. See nigripes Fallen..... 506 See vulgaris Fallen........ 575 (Calyptidia) ............ 517

Rondani. See echinura Desvoidy... 608 occulta Macquart (Exoprosopa, Heteralonia).. 551

Meigen (Limnobia, A malopis)........ 505

(Myopa, Gonirhyncus)...... 547

ocellaris Meigen ( Trichoptera, Phalænula) .... 587 ocellata Fabricius (Dictya, Pterocalla)....... 597 octopunctata Coquebert (Musca, Myennis).. $\quad 572$ Say (Dioctria, Taracticus)..... 612 oculatus Fallen (Dolichopus, Diaphorus)..... 532 œstriforme Brauer \& Bergenstamm (Hemi-

thrixion) ........................... 550 œstroidea (Desvoidy Crameria) ............. 528 (Crameria, A msteinia).. 506 (Crameria, Semiomyia). $\quad 605$

oleracea Linnæus (Tipula)............... 615 olivaceus Loew (Dasypogon, Triclis)......... 616

(Triclis, Gastrichelius)....... 546 omissa Schiner (Elliptera)............... 537 onopordi Desvoidy. See artemisiæ......... 545 opaca Loew (Syndyas) .................. 610 opilio Osten Sacken (Tanypremna)......... 612 oralis Desvoidy (Adia) ................. 503 oratorio Fallen ( Tachydromia, Microdromya). $\quad 569$ orbiculata Felt (Cecidomyia, Obolodiplosis).. $\quad 577$ oregona Aldrich (Siligo).................... 605 ornata Haliday (Medetera, Leptopus)....... 560 Johnson (Heterochroa, Spilochroa)..... 607 Meigen (Gonia, Reaumuria)........ 599 ornatipes Townsend. See pallipes Loew.... $\quad 517$ ornatum Meigen (Simulium. Atractocera).... 512

(Simulium, Melusina)..... 567

ornatus Haliday (Porphyrops, Xanthochlorus) 620 Macquart (Micropalpus, Cryptopalpus) ............... 528

(Micropalpus, Saundersia)................. 603

Meigen. See pedissequus Harris.... 620 (Ceratopogon, Bezzia)....... 513 oscinina Fallen (Madiza)................. 564 (Oscinis, Eurinella)......... 543 (Oscinis, Siphonella)......... 606 ovina Linnæus (Hippobosca, Melophagus)... 567 (Hippobosca, Melophila)..... 567 ovis Linnæus (Estrus) .................... 578 (Estrus, Cephalemyia)........ 519 oxyrhina Pandelle. See incana Fallen..... 612 pachygaster Fallen (Sargus, Neopachygaster). $\quad 575$ pachyneura Giglio-Tos (Tauromyia)........ 612 pacta Meigen. See cinerea Fallen......... 544 pagana Fabricius ( Musca, Mydæa)......... 572 palans Giglio-Tos (Chætocœlia) ............ 521 pallida Fabricius (Musca, Rohrella)......... 601 
Page.

pallida Fallen (Cordilura, Megaphthalma).. Macquart. See cinerea Desvoidy....

Meigen. See cervi Linnæus......... pallidipalpis Desvoidy (Blondelia, Lambertia) pallidiventris Meigen (Borborus, Lotobia).... pallidula Wulp (Brachycoma, Comatacta).... pallidus Coquillett (Demoticus, A pachemyia). Meigen. See pallipes Fabricius..... pallipes Fabricius (Musca, Plectropus)...... Loew (Platypeza, Calotarsa)......... Macquart (Agromyza, Phyllophila)... Meigen (Asilus, Mochtherus)......... (A silus, Neomochtherus)..... (Mycetobia)............... ( Mycetobia, Micetoica)....... See parvula Harris..........

palpalis Coquillett (Opsiomyia) ............. palpiger Coquillett (Lispidea)............... paludicola Skuse (Procladius) ............... palustris Desvoidy. See cinerea Fallen.....

(Limnophora) .............

Fallen (Ephydra, Coenia) .......... pandora Fabricius. See capucina Fabricius.. pantherina Linnæus ( Mfusca, Nemotelus)... papatasii Scopoli (Bibio, Cyniphes)..........

(Bibio, Flebotomus).........

(Bibio, Hæmasson) ......... paradoxa Osten Sacken (Cryptolabis)........ paradoxus Jænnicke. See unicolor Curtis... Meinert (Oligarces).............

Mik (Aptilotus).................. parasiticus Gervais (Trichobius)............ parisiensis Desvoidy ( $A$ mina $). . . \ldots \ldots \ldots \ldots$. parva Desvoidy (Egle).

Townsend (Lixophaga)

parvipalpis Wulp (Prospherysa, Plagiprospherysa) .......................... parvipes Townsend (Hypertrophocera)....... parvula Harris ( Musca, Chlorosia)........... ( Musca, Clorisoma) ...........

Loew. See gurgus Walker.......... pascuorum Meigen (Musca, Pararicia) pavo Aldrich (Leptocorypha).

pavonia Osten Sacken. See fascipennis Say. pavoniæ Desvoidy. See sylvatica Fallen... pectinata Hendel (Procreta).

Macquart (Ptychoptera, Ctenoceria).

Meigen (Agromyza, Agrobia)....... pedella Fallen ( Musca, Centrocera).......... pedissequus Harris ( Musca, Xanthogramma). pellucens Fallen ( Tachina, Eliozeta).........

Linnæus ( Musca, A pivora)......... (Musca, Pterocera)....... ( Musca, Volucella)....... pellucida Meigen ( Tachina, Nemoraea)...... pellucidus Coquillett (Nebritus).

pelops Melander (Oreothalia)............... pendula Linnæus ( Mrusca, Heliophilus)...... penelopes Weyenberg (Lynchia) ............ penicillata Rondani (Onodontha) ........... pennipes Linnæus (Empis).

peregrinum Meigen ( $A$ nthomyia, Euryomma). peregrinus Loew (Helophilus, Mesembrius).. pergandei Coquillett (Apocephalus).

Williston (Euceratomyia) . . perpusilla Six. See galeata Haliday......... perspicillaris Loew (Eclimus)..............
Page. $\begin{array}{lll}\text { perspicillatus Costa (Opsebius) .............. } & 580 \\ \text { perturbans Walker (Culex, Coquillettidia) } \ldots . . & 527\end{array}$ Williston (Aëdes, Isostomyia)..... 556 pertusa Meigen (A nthomyia, Helina) ........ 550 pertusus Loew. See pulla Wiedemann...... 505 perversus Brauer and Bergenstamm (Cnepha-

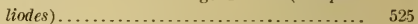

petiolata Desvoidy. See rustica Fabricius.. 555 Townsend (Euthyprosopa) ......... 543 Wiedemann (Dexia, Cordylidexia).. . 527 (Dexia, Cordyligaster). $\quad 527$ petronella Linnæus ( Ifusca, Calobata)....... 517 (Musca, Trepidaria)..... 615 phæoptera Meigen (Anthrax, Mima)........ 570 phalænoides Fabricius. See alternata Say... 616 Linnæus ( Tipula, Psychoda)... 597 pharœensis Theobald (A nopheles, Cellia)..... 519 phasiana Townsend ( Trichiopoda,Pennapoda) 5st; philadelphicus Macquart (Proctacanthus).... 595 phœenicurus Loew (Dizonias).............. 534 photophila Felt (Cecidomyia, Giardomyia)... 546 phryganopterus (Kolenati, Rypholophus) .... 602 phyllocerus Bigot (Phyllomydas).......... 590 phyllostomatis Perty (Lipoptena, Lepopteryx). $\quad 559$ pica Macquart (Leucomelina)............. 56 i0 piceus Walker (Pseudorus)............... 596 picipes Brauer and Bergenstamm (Erythran-

dra). picta Fabricius (Musca, Camptoneura)...... 518

(Musca, Delphinia)......... 531

Meigen (Lasioptera, Diomyza)......... 533

Wiedemann (A canthomera)........... 502 picticornis Loew (Scleropogon)............. 604 Townsend (Chætoglossa)........ 521 pictipennis Meigen (Limnobia, Limnophila).. 561 Wulp (Pseudomorinia) ......... 596 pictitarsis Bigot (Laparus, Chrysoceria)..... 523 pictus Bigot (Tetradiscus) .............. 613 Coquillett (Sinophthalmus)......... 605

Meigen (A silus, Bactria)........... 513 pilatei Macquart (Megistopoda)............ 5666 pilicornis Zetterstedt (Limnobia, Pilaria).... 591 pilifer Fabricius (Nerius) . ................. 575 piligena Rondani (Sphixapata, A rrenopus)... 510 pilipennis Fallen (Tachina, Thryptocera)..... 614 pilipes Haliday (Cœlopa) ................. 526 pilosa Desvoidy (Themira)............... 614 (Themira, Halidaya)....... 549

Meigen (Sciophila, Lasiosoma)........ 558 Zetterstedt (Cordilura, Orthacheta)... 581 pilosella Osten Sacken (Limnophila, Ulomor-

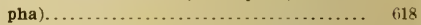
pilosellus Loew (Dasypogon, Daulopogon).... 531 (Dasypogon, Lasiopogon)..... $55 \mathrm{~s}$ pilosus Coquillett (Achætomus) ........... 502 pinguis Gerstæcker (Ectyphus) ........... 536 pini De Geer ( Tipula, Cecidomyia).......... 519

( Tipula, Cryptodiplosis)......... 528 (Tipula, Diplosis)............. 534 (Tipula, Itonida) ............ 556 piniariæ Hartig. See nigripes Fallen....... 546 pipiens Linnæus (Culex).................. 529

( Musca, Coprina).......... 527 (Musca, Syritta).......... 611

Pérris. See heteroneura Meigen..... 612 plagioides Townsend (Goniochæta)......... 547 
3age.

planiceps Fabricius (Damalis, Xenomyza) ... $\quad 120$

Loew (Xestomyza, Metaphragma). 568 planiventris Wiedemann (Rhaphiorhynchus) platyptera Panzer (Empis, Dionnæa) ........

(Empis, Platyptera)...... platypterus Fabricius (Dolichopus, Psilopodinus) .............

(Dolichopus, Psilopodius $). . . . . . . . . . . .$.

(Dolichopus, Psilopus) (Dolichopus, Sciapus).

Loew (Diogmites) ............... plebeia Desvoidy. See strigosa Fabricius... plebeja Linnæus ( Musca, Thereva).......... plebejus Fallen ( Tachina, Demoticus).......

(Tachina, Entomobosca).....

Loew (Hippelates)................ pleuropunctata Wiedemann (Ropcl o m e r $a$, $R$ ho $p$ al $l$ myia)........

(Ropalomera, W i 11 is to niella)........

plumbella Meigen ( Medetera, Oligochærtus)... plumicauda Aldrich (Xanthina)............. plumiger Meigen (Chlorops, Macrostyla).... plumipes F abricius ( Thereva, Trichiopeda). .

(Thereva, Polistomyia)...

Fries ( Tanypus, Anatopynia)..... plumosa Linnæus ( Tipula, Chironomus)..... (Tipula, Tendipes)......

plumula Loew (Lophoteles)................... pluricellata Schiner (Rhinotora)............ pluvialis Linnæus (Musca, A nthomyix)..... .

(Tabanus, Chrysozona)... ( Tabanus, Hæmatopota).. podacina Desvoidy. See fulvipes Desvoidy . podagra Newman. See globosus Fabricius.. podagrica Fabricius (Dacus, Merodin $x$ )......

(Dacus, Richardia)...... (Syrphus, Ascia)........ podomyia Brauer and Bergenstamm. See amica Meigen

podophyllæ Felt (Dicrodiplosis, Youngomyia) pœcilogaster Loew ( Trypeta, Blepharonetira). pokornyi Stein. See mikii Strobl. ........... polita Coquillett (Acicephala)...............

(Zabrachia)................

Linnæus (Musca, Chrysomyia)....... . (Musca, Microchrysa).......

(Musca, Myochrysa).........

Townsend (Epigrimyia)............. (Gymnoprosopa)........... politus Say (Dasypogon, Pygostolus)........ pollinosa Williston (Drosophila, Paratissa)...

WuIp (Hystricia, Eujuriniz)....... pollinosus Williston (Arthroceras) ........... polyodon Meigen (Tachina, Moschusa)........ polypori Loew (Diplosis, Coprodiplosis)...... polystigma Meigen (Anthomyiz, Brontæa)....

(Anthomyia, Gymnodia).. pomonæ Fabricius ( Tipula, Pullata)........ popelii Portschinsky (Echinomyix, Chxtopeleteria)..............

(Echinomyix, Popelia)... posticalis Lundbeck ( Tanypü, Trichotanypus) ................................. posticata Fabricius (Eristalis, Imatisma)..... posticatus Wiedemann (Culex, Conchylizstes). præcatoria Fallen ( Tachydromia, Chelifera).

( Tachydromia, Mantipeza).

(Tachydromia,Polydromys).

præceps Meigen ( Tachina, Pareudora)....... prasinus Loew (Diostracus) ................ pratensis Desvoidy ( A lina) See læta Fallen.......... 542

See polystigma Meigen .. $\quad 548$ pretiosa Loew (Himercssa).............. 552 p:ima Brauer and Bergenstamm (Ceratochæta)............................ 519 princeps Austen (Bogeria) ............... 515 proboscideus Fabricius (Tabanus, Pangonius) $\ldots \ldots \ldots \ldots \ldots \ldots \ldots . \quad 583$ Williston (Hippelates, Siphomyia $)$.....................

age.

\section{6}

555

565

593

585

34

prosopoides Brauer and Bergenstamm.

See pullata Meigen.................. 617

pruinosa Wheeler (Drepanomyia)........... 535 pseudohystricia Brauer and Bergenstamm

(Hystrisyphona, Echinodexia)............ 536

psilogaster Wiedemann (A silus, Atractia).... 572 psilcpterus Kiefier (Orthocladius, Psectrocla-

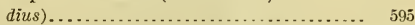

pubera Linnæus (Musca, Cordilura)........ $\quad 527$

(Musca, Mosina)......... 571

Loew (Echthodopa)............... 536

pubescens Loew (Prionccera).............. 594

(Prionocera, Stygeropis)..... 609

puelia Rondani (Istoglossa)............... 556

Wiedemann (Laphirix, Atomosia) ..... 572

pulchella Rossi (Musca, Ocneros) .......... $\quad 577$

(Musca, Toxonevra)........ 615

pulcherrima Arribalzaga (Uranotænia)...... 618 pulchra Schiner. See umbripennis Meigen.. 552 pulicaria Fallen (Empis, Atelestus)......... 511

(Empis, Platycnema)........ 592

pulicaris Linnæus (Culex, Culicoides)....... 529 pulicarius Mikan (Bombylius, Phthiria)..... 590 pulla Coquillett (Mauromyia)............. 565

Wiedemann (Trypeta, Amphicnephes). 505 pullata Meigen (Tachina, Tritochrta)....... 617 punctata Desvoidy (Ormia) ............. 580 (Soplia, Scotiptera)..... 604

Fabricius ( Miyopa, Dalmannia)..... 530 (Myopa, Stachyniz)...... 608

Latreille. See pulicaris Linnæus... $\quad 529$ punctatus Fabricius. See diadema Fabricius 521 punctigera Coquillett (Parcedopa).......... 585 punctipennis Am Stein. See œstroidea Desvoidy ................... 506

Meigen. See cincta Fabricius.. 595 See hybrida Meigen... 550

Say (Corethra, Sayomyia)....... 603 punetipes Meigen (Cordilura, Chætosa)..... 521

Proc.N.M.vol.37-10-41 
punctipes Wiedemann (Chironomus, Tanytarsus) $\ldots \ldots \ldots \ldots \ldots \ldots \ldots \ldots$ punctulata Williston (Nausigaster)......... puparum Fabricius ( Iusca, Meriania)......

( Musca, Platychira).... purpureus Walker (Hybos, Euhybus)........ pusilla Fallen (Copromyza, Coproica)........ ( Copromyza, Heteroptera)......

Meigen (Phasia, Hyalomya)......... Wiedemann ( Tachina, Sarcophilodes). pusio Loew (Chrysotimus).. putris Desvoidy. See eylindrica Fabricius

Linnæus ( Musca, Cheligaster).

pygophora Schiner (Salpingogaster)........ pyragra Zeller (Asilus, Tolmerus) ........... pyrastri Linnæus (Musca, Catabomba)....... ( Musca, Lasiophthicus).... (Musca, Scaeva) .......... pyrrhaspis Wiedemann ( Tachina, Tropido $\Gamma^{-}$

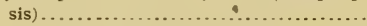
quadrifasciata De Geer ( Conops, Sphyxosoma) Fabricius ( Ifusca, Neurigona) quadrifaseiatus De Geer (Conops, Conopejus) Say (Paragus, Mixtemyia).. quadrifilum Loew (Rhaphium, Xiphandrium) quadripunctata Linnæus ( Musca, Sapromyzosoma)

quadripustulata Fabricius ( Musca, Dorbinia) (Musca, W i $\mathbf{n}$ themia).

quadrum Fabricius (Musca, Mydina)...... ( Musca, Spilogaster).... quercus Binney (Cecidomyia, Arnoldia)...... radiata Schrank. See stellata Fuessly...... rudicum Fabricius (.Musca, Erigone)......... ( Musca, Varichaeta)..... raptor Haliday (Aphrosylus).....

Macquart. See praecatoria Fallen... rebaptizata Rondani. See aenea Meigen..... rectinervis Desvoidy (Orillia)............... reflexa Desvoidy. See lateralis Fallen....... regalis Desvoidy (Chrysomya)............ (Chrysomya, Paracompsomyia).................

(Clirysomya, Pycnosoma)...

Rondani. See marklini Zetterstedt.. regina Meigen ( Musca, Phormia)........... replicata Linnæus ( Tipula, Phalacrocera)... raticulata Dumeril. See coryleti Scopoli..... rhingoides Bigot. See lineata Fabricius.... rhodophaga Coquillett (Neocerata)......... rileyi Coquillett (Mythicomyia) ............. riparia Desvoidy. See limosa Fallen........

$$
\text { (Melina). }
$$

Fallen (Ephydra) -iparius Meigen (Porphyrops, Perithinus).... rivosa Linnæus ( i i pula) Pedicia).......... rivularis Desvoidy. See livens Fabricius.... robertsonii Townsend (Euryceromyia)....... (Siphoclytia).......... iobusta Townsend ( Tachinomyia)... Wiedemann ( Tachina, Sphyromyia). robustus Osten Sacken (Comastes). röderi Girschner (Psilopa, Diasemocera).... Williston (Hysterichodexia, Euchae-

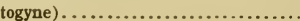

Page.
Page

roralis Linnæus ( Musca, Illigeria)......... 555

(Musca, Melanophora)..... 567

rorida Fallen (Sápromyza, Lycia).......... 563

rosarum Fabricius (Syrphus, Pyrophaena)... 599

rossii Giles (A nopheles, Grassia)........... 548

(Anopheles, Myzomyia)......... 573

rostellata Loew (Plecia, Rhinoplecia)....... 600

rosirata Bellardi. See rostellata Loew...... 600

Coquillett (Argyrophylar, Siphosturmia) .............................. 606

Desvoidy. See vertebrata........ 621

Linnæus. See austriaca Meigen.... 600

Zetterstedt. See immaculata Haliday ...................... 569 rostratus Linnæus ( Tabanus, Philoliche)..... 588 rotundata Linnæus (.Musca, Rhodogyne).... 600 (Musca, Gymnosoma)... 548 rotundiventris Fallen ( Tachina, Subclytia)... 610 rubens Coquillett (Eucessia)............. 541 rubiginosa Loew. See grandis Bergroth.... 552 rubra Felt (Johnsonomyia) . . . . . . . . . . . 556 rubricornis Desvoidy (Paykullia).......... 585 rubricosa Meigen ( Tachina, Frauenfeldia).... 545 ( Tachina, Tricogena)....... 616 rubriventris Macquart. See tibialis Desvoidy 525 (Senotainia)......... 605 Wulp (Mystacella, Mystacomyia) $\quad \$ 73$ rudis Fabricius (Musca, Cephysa) ......... 51 ) (Musca, Orizia).......... 580 ( Musca, Pollenia)......... 593

Fallen ( Tachina, Ernestia)........... 540 ( Tachina, Panzeria).......... 583

rufa Fallen (Heleomyza, Suillia).......... 610

Williston. See ferruginea Fallen....... 541

Wulp. See bilimekii Brauer and Bergenstamm....................... 575 rufata Bigot (Exorista, Bolomyia)......... 515 rufibasis Osten Sacken (Limnoplila, Priono.

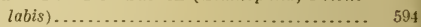
ruficauda Wulp (Schineria, Copecrypta)..... 527 ruficornis Fabricius (A silus, Cyrtopogon)..... 530 Macquart (Anisotamia) .......... 507 (Heteracanthia)........ 551 ( Micropalpus, Cuphocera)............ 529

( Micropalpus, Palpibraca)................. rufilatera Rondani (Exorista, Masipoda)..... rufipalpis Macquart. See leucophrys Wiedemann ............................. 514 rufipalpus Wiedemann( $\mathrm{X}$ ylophagus, Diphysa) 534 rufipennis Macquart ( Microphalpus, Epalpus) 539 rufipes Fabricius (Conops, Physocephala).... 590

Macquart (Eumetopia)............ 542 (Eumetopia, Eumetopiella). 542 (Xiphidicera)............ 621

Meigen (Cephalia)................. 519 (Cephalia, Myrmecomya).... 573 (Ephydra, Ephydrosoma)..... 538

Wulp (Homogenia).............. 553 (Homogcnia, Trichopododes).... 616 rufitarsis Macquart (Aulacigaster).......... 512 (Aulacigaster, Ampycophora)................ (A ulacigaster, A potomella ) ........................... rufithorax Wiedemann (Limnobia, Furina).

\section{6 509} 546 
rufiventris Maequart. See abdominalis Say . (Megaprosopus)....... Meigen. See canescens Meigen... rufum Brauer and Bergenstamm (Cyrtosoma) rugonasus Williston (Melanostomn, Rhysops). ruralis Fallen ( Tachina, Plagia) .............

(Tachina, Voria) .............

Meigen. See femorata Fabricius..... ruricola Meigen ( Tachina, Cyrtophleba) ....... rustica Fabricius ( Musca, Dexia)............

( Musca, Dexilla)...........

( Musca, Ida $). . . . . . . . .$.

Meigen. See simulans Meigen ........

Winnertz (Phronia)................ rutila Meigen ( Tachina, Ceromasia)......... Wiedemann (Anthrax, Trinarix)...... rutilioides Jaennicke (Dejeanin, Paradejeania) rutilus Wiedemrann (Dasypogon, Dicranus).. sabaudus Fabricius ( $A$ silus, Stenopogon) ...... sabulicola Loew (Laphystia).

sabulonum Osten Sacken (Clavator, Lesto-

myia)

sabulosa Desvoidy (A nicia).............. salax Wheeler (Peloropeodes)..

saliciperda Dufour (Cecedomyia, Dichelomyia). salicorniæ Kieffer (Baldratia) .

saltatoria Desvoidy. See podagrica Fabricius saltatrix Desvoidy. See sealaris Fabricius...

Linnæus ( Musca, Mcromyza)...... saltuum Fabricius (Syrphus, Spilomyia) ..... sanctipauli Sehiner (Telmatogeton).......... sarcinata Loew ( Trypeta, Peronyma)......... sarcophagina Brauer and Bergenstamm (Myiomima) ..............

Townsend (Laccoprosop 7 ).... sarothamni Loew (Cecidomyia, Asphondylia). satanica Bigot (Scopolia, Penthosia)......... saundersii Curtis. See hybrida Meigen..... saxicola Osten Sacken (Antocha) . . ........... sayi Aldrich (Odontopoda)..................

Felt (Epidiplosis). scævoides Fallen (Rhingia, Chamaesyrphus). scalaris Fabricius ( Musca, Fannia).. scambus Fallen (Dolichopus, Camptoscsles). scapularis Loew (Hemerodromia, Neoplasta).. scatophora Perris ( Mycetophila, Epicypta).... schineri Kolenati (Crunobia)............... schistacea Meigen. See obscura Fallen..... schnablii Brauer and Bergenstamm (Petinops) sciarina Meigen (Zygoneura).

scintillans Loew (Psilopus, Gnamptopsilopus) scita Harris (Musca, Milesiformis).

$$
\text { (Musca, Tropidia)............... }
$$

scolopacea Linnæus ( Musca, Leptis)......... ( Musca, Rhagio)....... . seorzoneræ Desvoidy (Sitarea)............... seripta Linnæus ( Musca, Melithreptus)......

(Musca, Melitrophus)......

(Musca, Sphærophoria) ....

serobiculata Loew (Acrosticta)............. scutatus Meigen (Syrphus, Platycheirus)..... scutellaris Desvoidy ( $A$ medea).

See pagana Fabricius.. See radicum Fabricius. scutellata Desvoidy ( Nemoræa, Blepharipa).. (Nemoræa, Ctenocnemis).
Page.

534

566

613

530

601

591

619

582

530

532

532

555

521

589

520

617

584

533

608

557

560

506

586

533

513

601

544

567

607

612

587
576

Marquart (Volucella, Atemnocera).. 511

Winnertz (Corynoneura).......... 528

scutellatus Bigot (Doliosyrphus) ........... 535

scybalaria Linnæus ( Musca, Scatomyza)..... 603

securicornis Fallen (Phyllomyza)............ 590

segmentaria Fabricius ( Musca, Mya)....... 571

(Musca, Somomya)... 606

segnis Linnæus ( Musca, Eumeros).......... 541

(Musca, Micraptoma)...... 569

(Musca, Xylota)........... 621

(Musca, Zelima)........... 621

segregata Rondani (Chetogena, Duponchelia). $\quad 536$

(Chetogena, Parasetigena). 585

selene Osten Sacken (Anthrax, Thyridanthrax) 615

semichrasti Townsend (Euacaulona)........ 540

semiglauca Perris. See viridis Meigen...... 508

semilucifera Villiers ( Musca, Sphixea)........ 607

seminationis Linnæus (Musca, Megaglossa).. 566

(Musca, Platystoma).. 592

semiviridis Wulp (Chloroprocta) . .......... $\quad 522$

senilis Fabricius. See fenestralis Linnæus... 555

sepia Meigen (Anthomyix, Chortophila)...... 523

septemguttata Kieffer (Leptodiplosis) ........ $\quad 559$

sepulchralis Linnæus ( Musca, Eristalinus)... 540

Meigen ( Musca, Onesia)....... 579

sequens Townsend (Vandcrwulpia, Catemo-

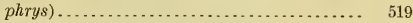

seriata Loew ( Trypeta, Icterica)............ $\quad 555$

sericariæ Rondani ( Ugimyia).............. 618

(Ugimyia, Crossocosmia).. 528

sericata Meigen (Glochinx) ................ 547

(Glochina, Sixgona).......... 605

(Musca, Phenicia)........... 588

serpentina Osten Sacken (Dipalta) ........ 534 serpentinus Wiedemann (Dacus, Anastrepha) 506 serrata Desvoidy. See irritans Linnæus..... 594 Linnæus ( Musca, Blephariptera)..... 514

( Musca, Heleomyza)....... 550

(Musca, Leria)............ 560

serratulæ Linnæus ( Musca, Terellia)........ 613

serratus Theobald (Culex, Protoculex)....... 595

serriventris Rondani. See concinnata Mei-

gen .............................. 563

serva Desvoidy $($ A myclæa $) \ldots \ldots \ldots \ldots \ldots \ldots .506$

servillei Macquart (Phoneus).............. 588

(Phoneus, Neophoneus)... 575

setacea Becker. See macquarti Zetterstedt). 608 seticornis Fallen (Lauxanix, Pachycerina).... 582

Wiedemann (Acanthomera, Megalomyia).

setifacies Brauer and Bergenstamm (Crypto-

meigenia) ........................... setigera Brauer and Bergenstamm (Pseudophorocera $) . . \ldots \ldots \ldots \ldots \ldots \ldots \ldots \ldots \ldots . .596$ setipennis Coquillett (Houghia).......... 553 setosa Coquillett (Chætophleps) ............ 521

(Phyto, Neophyto)......... 575

Desvoidy. See pilosa Desvoidy....... 549 setosus Fabricius (Syrphus, Platynochætus).. 592 sexdentata Fabricius. See chalybeata Fors-

ter ............................. 513, 552 siberita Fabricius (Stomoxys, Prosena)...... 595 (Stomoxys, Calirrhœ) ...... 517

sibirica Loew (Arthropeas)............. 510 sicanus Costa. See diadema Fabricius...... 605 
Page.

sicula Desvoidy (Rhedia, Isomera)... Rondani (Spathulina).

signata Meigen ( Tachina, Pachyophthalmus). signifer Coquillett ( Culex, Pneumaculex).... signifera Wulp (Cenosoma)............... silvatica Desvoidy (Egeria)................

See puparum Fabricius... See vulgaris Fallen.

silvestris Desvoidy. See vulpina Fallen. silvicola Curtis. See albipes Strom... simplex Fallen (Ocyptera, Leucostoma)....

(Ocyptera, Psalida).........

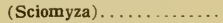

(Sciomyza, Bischofia).......

Loew (Dicolonus).

Macquart (Erax, Eicherax)...

Walker (Syneches)....

simplicitarsis Zetterstedt. See aenea Meigen. simulans Meigen ( Tachina, Adenia).........

( Tachina, Bigotia)..........

( Tachina, Chaetotachina)....

(Tachina, Cleodora)........

(Tachina, Eribea)........ 539

( Tachina, Esila).......... 540

(Tachina, Futilia)........ 540

( Tachina, Gaubilia)....... 546

(Tachina, Walkeria)....... 619

( Tachina, Zelleria)......... 621

( Tachina, Zetterstedtia).... 622

singularis Burgess (Glutops)

Schiner (Hilarimorpha).

Townsend (Atrophopoda)....... $5 \tau^{2}$

(Atrophopoda, Lachnomma)...

Williston (Probolaeus)...

siphonina Bigot (Proboscimyia).

(Proboscimyia, Dolichoglossa) skinneri Coquillett (Trochilodes)...

slossonæ Coquillett (Lipochaeta)

(Nostima).

smaragdinus Gerstaecker (Eulonchus)

(Thrypticus)......

sodalis Osten Sacken (Cophura)........... solenopsidia Brues (Commoptera)......... solidaginis Fitch (A cinia, Eurosta)......... solita Wulp (Mystacella)...

solstitialis Fallen (Eristalis, Chrysogaster). Linnæus ( Ifusca, Tephritis). . sonchi Desvoidy. See cardui Linnæus. Linnæus ( Musca, Ensina).. sorbi Kieffer (Contarinia, Eudiplosis). sparsa Wiedemann ( Trypeta, Eutreta)...

(Trypcta, Icaria)........

speciosa Meigen (Milichia).

spectabilis Meigen. See albisquama Zetterstedt

See plebejus Fallen.

Wulp (Melaleuca)........

sphegeus Fabricius (Syrphus, Sepedon)

sphyricera Macquart (Echinomyia, Sphyricera) spilota Curtis (Ephydra, Epipela).

$$
\text { (Ephydra, Ilythea). }
$$

spinifera Leach (Feronia).

\section{(Feronia, Olfersia)......}

spiniger Wiedemann ( $X$ ylophagus, Exaireta).

(Xylophagus, Exaireta).

9

0

st

stagnicola Desvoidy. See coarctata Fallen. stellata Fuessly ( Musca, Trupanea)......... ( Musca, Urellia)........... stercoraria De Fieer ( $T$ ipula, Orthocladius).. Desvoidy (Nemopoda, Meroplius). stercorarius Meigen (Borborus, Cimbometopia) sternodontis Townsend (Sarcodexia)......... stietica Meigen (Limnobia, Symplectomor pha). stigma Fabricius ( Musca, Notogramma).... Meigen ( Myopa, Myopella).......... stigmatica Osten Sacken (Empeda)......... strenua Desvoidy. See strigosa Fabricius... striata Meigen (Sciophila, Empheria)........ (Sciophila, Neoëmpheria)..... striatus Fabricius (A silus, Dactiliscus)...... strigosa Fabricius ( Musca, Hylemya)........ (Musca, Musciosoma)..... strigula Fabricius ( Musca, Anthracophaga). . stylata Fabricius (Conops, Stylogaster)....... styriaca Pokorny. See bilbergi Zetterstedt.. subcoleoptrata Linnæus (Conops, Phorantha) submetallica Rondani (Phalacromya)....... subopaca Coquillett (Leucostoma, Euphyto).. subrotunda Desroidy. See ferina Fallen.... subsessilis Illiger (Ceria, Cerioides).......... (Ceria, Sphiximorpha)...... subsultans Fabricius ( Musca, Borborus).... ( Musca, Cypsela)...... 530 Sphærocera)... 607 (Syrphus, Glabrinus)... 547 (Syrphus, Gymnopa)... 548 (Syrphus, Mosillus).... 571

Page.

,
suillioidea Desvoidy (Herbina)........... 550 suillorum Desvoidy (Terenix).............. 1.13 sulcata Meigen (Empis, Rhamphomyia)...... 599 sulphureus Mikan (Bombylius, Systœchus)... 611 superbiens Schiner (Paltostoma) .......... 583 surda Zelterstedt. See exsurda Pandelle... 566 susperta Loew. See melanura Meigen...... 509 
Page.

suturalis Fallen (Dolichopus, Saucropus)..... 603 suturata Rondani (Clausicella)............ 524 sylvarum Linnæus (.Musca, Heliophilus)..... $\quad 550$ șlvatica Curtis (Sciophila, Tetragoneura)....

Fallen ( Tachina, Masicera)........ sjlvaticus Meigen (Borborus, Limosina)...... sylvestris Desvoidy. See segregata Rondoni..

Theobold (Culex, Ecculex)......... srlvicola Curtis. See albipes Strom..........

Walker. See pulicaria Fallen..... syngenesiæ Fabricius ( Musca, Rivellia)...... s5rphoides Panzer. See cinctus De Geer.... tabaninus Thunberg (Pantophthalmus)...... tachinoides Fallen ( Ifusca, Morphomya)...... tachinomoides Townsend (Euphorocera)..... tæniatus Bellardi (Rhyphus, Olbiogaster).... tænionota Meigen (Erioptera, Chenialida).... (Erioptera, Octavia)....... (Erioptera, Polyraphia).... tæniorhynchus Arribalzaga. See titillans Walker..................

Wiedemann (Culex, Culicelsa) tanaceticola Karsch (Oligotrophus, Rhopalomyia).

tandrec Desvoidy (Theresia)...............

(Theresia, Sardiocera)..... tarandi Linnæus (Cstrus, CEdemagena)..... tarsata Wahlberg. See rufitarsis Macquart.. tarsatus Fallen (Hydrochus, Synarthrus).....

Meigen. See splendida Meigen.... tauscheri Fischer'(Rhynchocephalus)........ tempestiva Fallen ( Musca, Byomya).......... tenax Linnæus ( Musca, Elophilus).......... (Musca, Eristalis)........... (Musca, Eristaloides)......... (Musca, Eristalomya)......... (Musca, Tubifera)........... tenella Meigen (Limnobia, Gonomyia)......... (Limnobia, Taphrosia)........ tenera Wiedemann (Stomoxys, Leskiomima). tentaculata De Geer ( Musca, Lispe)......... tentatrix Loew (Euthera) .................... tenuipes Bigot (Rhynchiodexia)..............

Osten Sacken (Rhophidolabis)......

tenuis Loew (Stenomyia) .................... tenuiventris Bigot (Macrosargus)............

(Macrosargus, Pedicella).

terrænovæ Desvoidy (Phormia, Protophor-

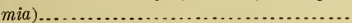
territans Walker (Culex, Neoculex)........... tessellata Brauer and Bergenstamm (Tetragrapha)

testacea Desvoidy. See bicolor Meigen...... See fimetaria Linnæus. (Palusia) ............... Linnæus (Conops, Phorosia)........ (Conops, Stomoxoides).....

Macquart (Plesiomma) ............. See devia Haliday.......

Ruthe. See devia Haliday.........

Wulp (Saundersia, Rhachopalpus).. testaceus Fabricius (Sargus, Ptecticus)....... Loew (Longurio)................... Macquart (Cyclorhynchus)......... testudinea Loew ( Trypeta, Acrotaenia)........ tetanops Loew ( Trypeta, CEdicarena)........ theotænia Wiedemann (Tabanus, Stibasoma) 609 thermophila Wiedemann (Tachina, Sisyropa) 606 theutis Walker ( Tachina, Emphanopteryx)... 537 thlipsomrzoides Jaennicke (Poecilognathus)... 593 thomæ Linnæus ( Tipula, Lycoria).......... 563

(Tipula, Molobrus)......... 571

(Tipula, Sciara)........... 604

thoracica Desvoidy (Cllorina) ............. 522 See picta Fabricius...... 531

Lioy ( Trisometopia).............. 617 thoracicus Loew (Pterallastes)............. 597 thymi Kieffer (Cecidomyia, Janetiella)...... 556 tibialis Desvoidy (Estheria, Clinoneura)..... 525

(Estheria, Ptilodexia)...... 598 (Herbstia) ................ 551

(Talmonia).............. 612

Fallen ( Tachina, Hyria)............. 555

(Tachina, Pelatachina)........ 586

Macquart. See quadripunctata Lin-. næus......................... 602

Meigen (Chironomus, Cricotopus)..... 528

Walker (Pheneus)................ 588

(Pheneus, A rthrostylum)..... 510

tiefi Mik. See argyragastra Perris........... 608 tigrina Fabricius ( Musca, Caricea)........... 518 tipuloides Bose (Keroplatus).............. 557 Fabricius. See cylindricus De Geer....................... 559

Linnæus. Seecylindricus De Geer. $\quad 547$ titillans Walker (Culex, Mansonia)......... 565 (Culex, Panoplites).......... 583 (Culex, Taeniorhynchus).... 612

tomentosa Desvoidy (Stevenia)............ 698

torrens Townsend (Tersesthes)........... 613

tosi Becker (Milichiella) . . . . . . . . . . . . . 570

townsendi Williston (Atrophopoda, Ede-

mapeza)............................. 578

toxicodendri Felt (Cecidomyia, Adiplosis).... 503

transfuga Linnæus ( Musca, A nasimyia)...... 506

transversa Felt (Asphondylia, Cincticornia).. ' 524

trapæ Desvoidy. See cinerea Fallen....... 557

trepida Meigen ( Tachina, Blepharigena)...... 514

(Tachina, Paraplagia)....... 585

triangula Fallen ( Musca, Pseudolimnophora). 596

(Musca, Stroblia) .......... 609

triangulifera Loew (Hyalomya, Hyalomyodes) 553

tricincta Meigen (Pelecocera).............. 586

tricolor Coquillett (Metadexia)............. 568

Zeller(Tabanus, Therioplectes)....... 614

trifarius Loew (Ablautus) ................. 502

trifasciata Meigen ( Trichoptera, Pericoma)... 587

Winnertz. See fasciata Meigen... 534

trigonus Meigen ( $A$ silus, Dysmachus) ........ 536

trilineata Fabricius. See hypoleon Linnæus. 582

trimaculata Fabricius (Scatophaga, Coilo-

metopia)............................ 526

trinotata Desvoidy. See maculata Meigen... 578

trinotatus Rondani. See maculata Meigen.. 505

tripartitus Aldrich (Phylarchus)............ 590

tripunctata Fabricius (Tipula, Amphinome) 505

(Tipula, Limnobia)... 561

(Tipula, Limonia)..... 561

(Tipula, Limnomyza). 561

(Tipula, Unomyia).... 618

Wiedemann (Anthrax, Argyrn-

moeba)................... 510

triquetra Meigen. See macquarti Stæger.... 512

\section{(1)}

7

1

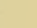

7

6

98

(n)

8

8

18
.

\section{(1)}


triquetra Olivier (Ocyptera, Ervia)..........
(Ocyptera, Paranaphora)....

Wiedemann (A nthomyia, Azelia)...

triseriatus Say (Culex, Protomacleaya)........ tristis Schiner (Mesorhaga)...............

Williston (Melanodexia)............ tristissima Osten Sacken (Gnophomyia)...... trisuleata Schummel (Limnobia, Triogma).. trivittata Coquillett (Culex, Pseudohowardina)...

Meigen (Leia, Boletina)........... trochilus Coquillett (Apomidas)........... trollii Zetterstedt (Aricia, Chiastocheta)....... trompe Linnæus (Oestrus, Cephenemyia).... (Oestrus, Endocephala)..... truquii Rondani. See rubricosa Meigen..... tuberculatus Macquart. See valgus Panzer. . tubifer Meigen (Sapromyza, Stylocoma)....... tumida Erichson (A crocera, Paracrocera)..... tussilaginis Fabricius ( Musca, Carpomya)... uber Giglio-Tos (Cyrtoneurina)...

uliginosus Linnæus. See pantherina Linnæus.

ulocoma Theobald (Dendromyia)

umbellataruin Fabricius (Musca, Palloptera) umbrarum Fabricius ( Musca, Hesyquillia). . umbratica Meigen (Anthomyia, Hebecnema). umbripennis Meigen (Rhamphomyia, Holoclera)...

undata Wiedemann (Pyrgota)....

(Pyrgota, Oxycephala)..

underwoodi Underwood (Eucorethra)

(Eucorethra, Pelorempis)...

undulata Winnertz (Polylepta).....

ungulans Pandelle (Miltogramma, Macroni-

chia)..............

ungulata Linnæus ( Musca, Dolichopus).... (Musca, Iphis)........ ( Musca, Satyra).

unica Stein ( Tetrachxta)

(Tetrachxta, Tetramerinx)

Townsend. See floridensis Townsend. unicolor Curtis (Atherix, Eurytion)...

Haliday (Geranomyia).

unifasciata Desvoidy (Latreillia, Triachora).. Macquart (Dichelacera)

unimaculatus Loew (Chasmatonotus).......

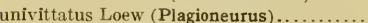

urbiea Curtis (Trichomyia) .............. ursus Costa. See gigas Herbst. ............ urticæ Fabricius. See polita Linnæus......

Linnæus (. Musca, A nacampta)...... (Musca, Ceroxys) ..........

Perris (Cecidomyia, Perrisia)......... ustulata Curtis (Helcomyza) . . . . . . . . . . . . vagans Loew (Diastata, Calopterella).......

(Pelastoneurus) ..............

Wiedemann (Xylota, Planes)........ vaginalis Fallen: See chorea Fabricius.... valgus Panzer (Syrphus, Brachypalpus)

valida Brauer and Bergenstamm. See tandree Desvoidy

Townsend. See parvipalpis Wulp....

Winnertz (Docosia)...

validinervis Wulp (Didyma, Paradidyma)...
Page.

540

585

512

595

568

566

547

617

596

515

509

522

519

537

616

516

610

583

518

530

574

531

583

551

549

552

598

582

$5+1$

586

593

564

535

555

603

613

613

602

543

546

615

533

521

591

616

516

573

506

520

587

549

517

586

591

562 516 validum Brauer and Bergenstamm (Mochlo-

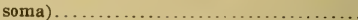
vandykei Coquillett (Spogosylum, Coquilletiia). ...................

stæger (Mycetophila, Zygomyia).......

aria Meigen (Erioptera, Dasyptera).........

Wulp (Distichona)..................

(Distichona, Olenochæta).........

variabilis Desvoidy. See disjuncta Wiedemann.................... 505

Loew (Chauna)................ 521

(Chauna, Neochauna)..... 574 Zetterstedt (Asilus, Rhadiurgus).. $\quad 599$

varicolor Coquillett (Plethochæta) . . . . . . . . 592

Meigen (A nthomyia, Botanophila).. 515

variegata Fallen (Drosophila, Phortica)...... 589

Meigen ( Myopa, Myopina)........ 573

Winnertz (Leia, Rondaniella)...... 601

varipennis Walker. See distendens Wiedemann ....................

Williston (Sphenoidoptera).......

varipes Coquillett (Pseudodinia)........... varus Panzer (Syrphus, Xylotæja)......... vau Say (Ortalis, Pseudotephritis).......... (Ortalis, Stictocephala) ............ vegetus Wheeler (Nothosympycnus).......... velox Desvoidy. See melanoptera Fallen... relutina Ruthe (Corethra, Mochlonyx)....... velutinus Macquart (Microphorus)......... venata Aldrich (Phora, Pachyneurella)....... venatica Haliday (Chionea, Epidapus)....... venatoris Coquillett (Demoticus, Neofischeria)

(Demoticus, Parafischeria).............. 584

venosa Wulp (Megaparia).............. 566 venosus Wiedemann (Dasypogon, Microsty-

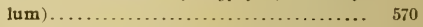
venusta Meigen (Ceratopoyon, Probezzia)..... 594

Rondani. See frontalis Loew ...... 518 vernalis Desvoidy. See guttata Fallen..... 553 See moerens Meigen.... 557 See obsoleta Meigen..... 573 versicolor Loew ( Chlorops, Diplotaxa)...... 534 vertebrata Say (Dexif, Zelia)........... 621 verticalis Meigen. See ruralis Fallen......... 591 vesiculosus Fabricius (Syrphus, Brachystoma) 516 vespertilionis Fabricius. See avium Macquart...................

Linnæus (Pediculus, Celeripes) 519 (Pediculus, Nycteribia) 577 vespertina Fallen ( Musca, Hydrophoria)..... 554 vespiformis Gorski. See vittatum Wiede-

mann................... 618

Linnæus ( Musca, Tritomia)..... 617 vespillo Fabricius ( Musca, Nitellia)........ 576 See sepulchralis Meigen... 579 vestita Wiedemann (Dolichopus, Leucostola). 560 vetula Fallen. See flaveola Fabricius....... 535 viarum Desvoidy. See erratica Fallen...... 587 vibrans Linnæus ( Musca, Myodinx)........ 573

(Musca, Ortalis)......... 581

(Musca, Seioptera)......... 605 viburni Felt (Cecidomyia, Dentifibula)....... 531 (Mycodiplosis, Karshomyia) .... 556 vicina Desvoidy. See atra Meigen........ 600 
Page.

vilis Wulp (Prospherysa, Chaetogaedia) .... 521 villosa Meigen (Heleomyza, Scoliocentra)..... 604

Rubsaamen (Rhynchosciara)........ 600

villosus Bigot (Merapioidus) .............. 567 (Romaleosyrphus)............ 601

riminalis Westwood. (Rabdophaga)......... 599

violacea St.Fargeau and Serville ( Temnocera). 612

Wulp. See rufata Bigot........... 515

virens Fabricius ( Iutio, Phalangus)........ 558 (Mulio, Pipizella).......... 591

Scopoli (Musca, Liancalus).......... 511 virescens Fabricius (Bombylius, Conophorus). $\quad 527$ (Bombylius, Ploas)......

Philippi (Plettusa)................ virginicaFelt (Dasineura, Camptoneuromyia). virgo Zetterstedt (Limnobia, Ninguis) viridescens Desvoidy. See chalconota Meigen.

viridis Fallen (Tachina, Gymnocheta) -...

Meigen (Medetera, Aphrozeta)........ vitinea Felt (Lasioptera, Neolasioptera)...... vitis Rübsaamen (Arthrocnodax)

vitripennis Meigen (Chlorops, Oscinisoma)... ( Ifusca, Plaxemya)...... (Phora, Gymnoptera).....

vittata Bellardi (Apelleia)

Haliday. See analis Meigen........

Macquart (Physegenua).............

Meigen (Mallota, Lejops).............

Schummel. See ferruginea Fallen...

Wiedemann (Chiromyza)............ vittatum Wiedemann (Chrysotoxum, Sphecomyia)............ (Chrysotoxum, Tyzenhausia).............

vittatus Bigot (A crochordonodes)............. vittigera Bigot (Cyrtonevra, Hemichlora).... (Homodexia, Thelairodes).... vittula-Loew. See albicosta Walker. rituli Fabricius (Tabanus, Silvius)........... vocatoria Fallen ( Tachydromia, Chyromya)... 524 volucris Osten Sacken (Eupeodes) .......... 542 volvulus Fabricius ( Irusca, Hypostena) ...... 555 vomitoria Linnæus. See erythrocephala.... 517 vulgaris Desvoidy. See equina Fallen...... 572 Fallen ( Tachina, Blepharidea)....... 514 (Tachina, Blumia) .......... 515 (Tachina, Erinia)........... 539 (Tachina, Eurigastrina)...... 542 (Tachina, Phryxe).......... 589

vulnerata Loew ( Trypeta, Stenopa)......... 608 vulpina Fallen ( Tachina, Linnaemya)....... 561 wahlbergi Zetterstedt (Oxyrhina, Acrometopia) 503 walkeri Curtis (Leptomorphus) ............. 560

Theobald (Culex, Howardina)...... 553 waltlii Brauer and Bergenstamm (Parapro-

sena)....................... 585

Meigen (Diamesa) ................ 532

weedi Coquillett (Neocota) ................ 574

Townsend. See triangulifera Loew... 553

wesmælii Macquart (Paramesia) ........... 584

westwoodi Guerin - Meneville (Nycteribia,

Penicillidia)............... 586

Osten Sacken (Elephantomyia).... 537

wheeleri Brues (Ecitomyia)............... $53 \dot{6}$

wiedemanni Meigen ( Trypeta, Goniglossum). $\quad 547$

(Trypeta, Orellia)..... 580

willistoni Townsend. See ælops Walker... 577

Wheeler (A phantotimus) ......... 508

winthemi Macquart. Seeferruginosa Meigen. 564

xanthogastra Wulp (Camarona)........... 517

xanthoptera Wiedemann (Laphria, Cero-

tainia)............................ 520

xanthostoma Walker (Prochyliza) ......... 594 josemite Osten Sacken (Blepharicera, Phi-

lorus $) \ldots \ldots \ldots \ldots \ldots \ldots \ldots \ldots \ldots \ldots \ldots . .588$ zonata Destoidy. See confinis Fallen...... 509 zonatus Erichson (Ogcodes) .............. $\quad 578$ 

Crond 


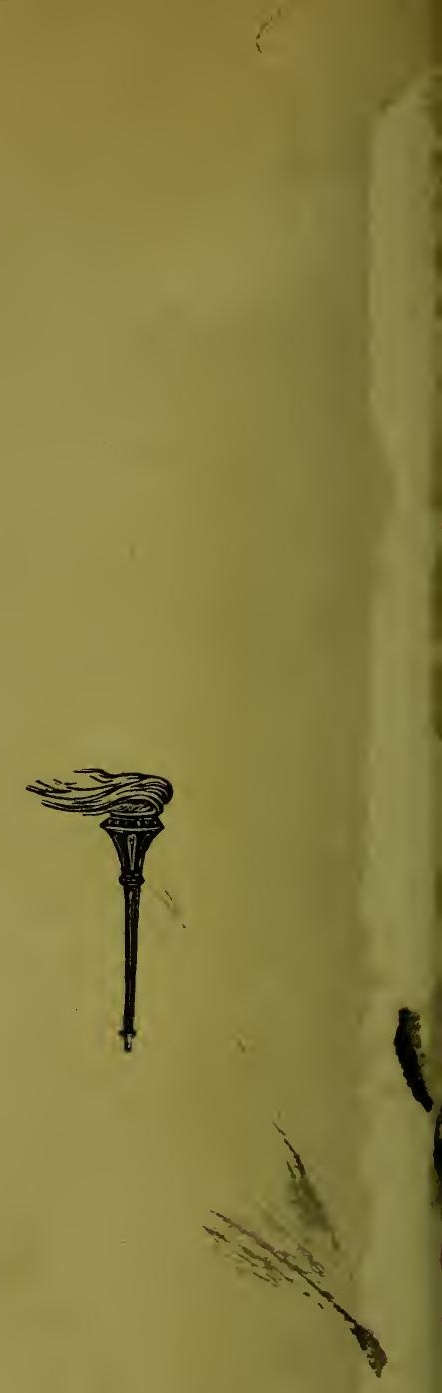







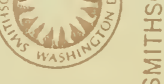

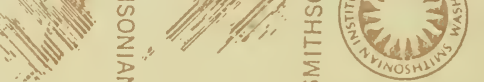
is
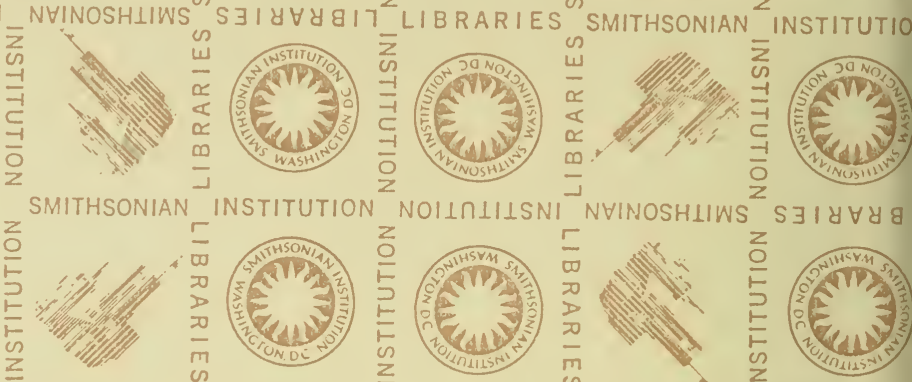

e
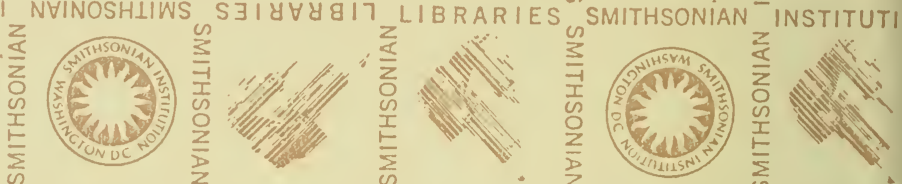

ARIES SMITHSONIAN
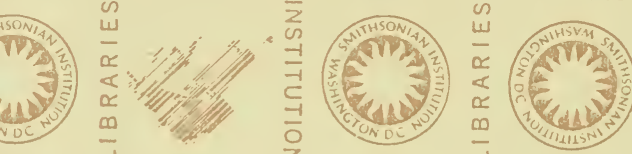
NVINOSHIIWS

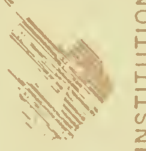

SMITHSONIAN
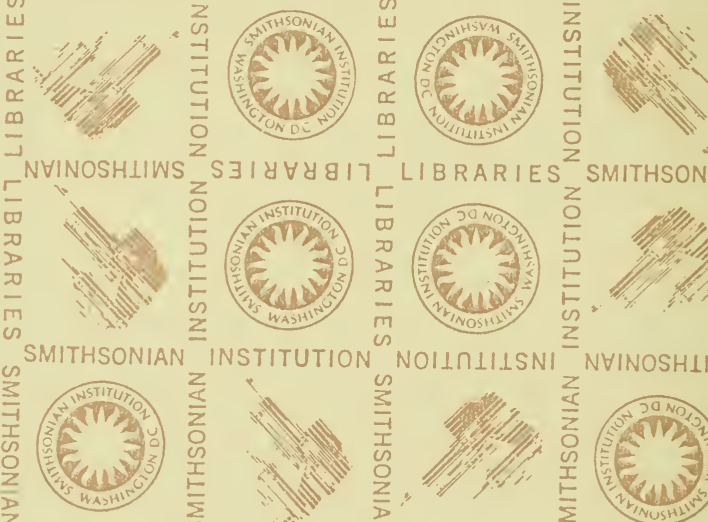

" $S \exists \mid 4 \forall \forall 81$

SMITHSONIAN
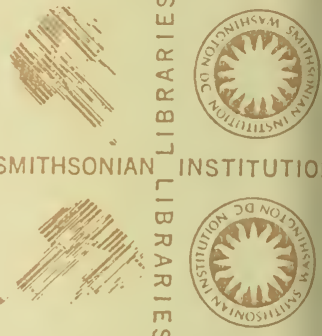

NSTITUTION NOILOHISNI
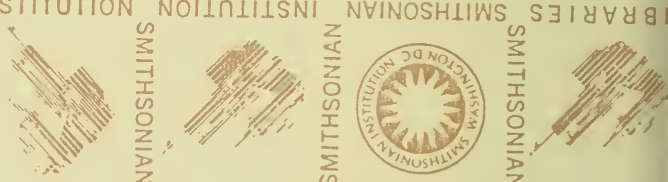

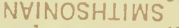
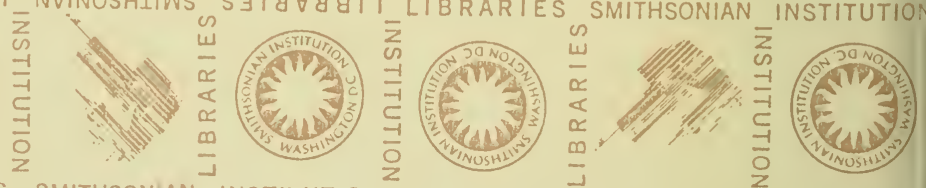

SMITHSONIAN
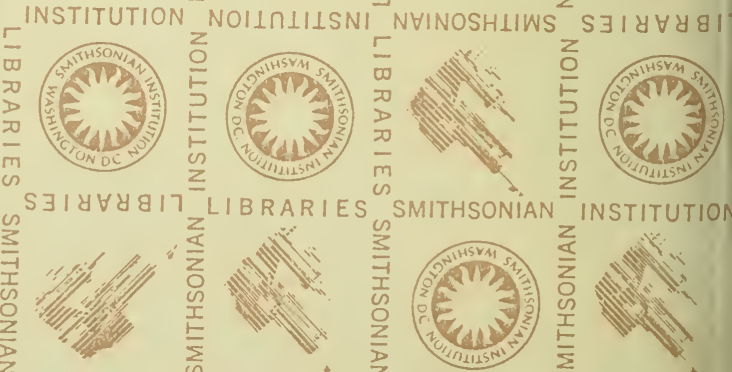

\begin{tabular}{l}
$z$ \\
$\frac{Z}{Z}$ \\
0 \\
s \\
$\frac{1}{E}$ \\
\hline
\end{tabular} 
